InTEgRATED RAMAN SPECTROMETERS FOR

ApPlications in Health and Medicine 
Alla mia bellissima famiglia... 



\section{INTEGRATED RAMAN SPECTROMETERS \\ FOR APPLICATIONS IN HEALTH AND MEDICINE}

NUR ISMAIL 


\section{Members of the Graduation Committee}

Chairman and Secretary

Prof. dr. ir. A. J. Mouthaan

Promoters

Prof. dr. M. Pollnau

Prof. dr. A. Driessen

\section{Assistant Promoter}

Dr. ir. R. M. de Ridder

\section{Members}

Prof. dr. Vinod Subramaniam

Dr. ir. Herman Offerhaus

Prof. dr. A. G. J. M van Leeuwen

Prof. Dr. P. Andersen
University of Twente

University of Twente

University of Twente

University of Twente

University of Twente

University of Twente

University of Amsterdam

Technical University of Denmark

The research described in this thesis was carried out at the Integrated Optical MicroSystems (IOMS) Group, Faculty of Electrical Engineering, Mathematics and Computer Science, MESA+ Institute for Nanotechnology, University of Twente, P.O. Box 217, 7500 AE Enschede, The Netherlands. The research was financially supported by the IOP Photonic Devices, managed by the Dutch NL Agency and the Technology Foundation STW.

\section{Cover design:}

Layout of an integrated confocal light delivery and signal collection device made out of two zero-order arrayed waveguide gratings.

\section{Copyright (C) 2011 by Nur Ismail, Enschede, The Netherlands}

All rights reserved. No part of this book may be reproduced or transmitted, in any form or by any means, electronic or mechanical, including photocopying, microfilming and recording or by any information storage or retrieval system, without the prior written permission of the author.

This thesis was printed by Wöhrmann Print Service, The Netherlands.

ISBN 978-90-365-3326-3

DOI 10.3990./1.9789036533263

URL http://dx.doi.org/10.3990/1.9789036533263 


\section{INTEGRATED RAMAN SPECTROMETERS \\ FOR APPLICATIONS IN HEALTH AND MEDICINE}

\section{DISSERTATION}

to obtain

the degree of doctor at the University of Twente, on the authority of the rector magnificus, prof. dr. H. Brinksma,

on the account of the decision of the Graduation Committee,

to be publicly defended on Wednesday $8^{\text {th }}$ of February 2012 at 16:45 hrs.

\section{NUR ISMAIL}

Born on the $9^{\text {th }}$ of May 1977

in Palermo, Italy 
This dissertation is approved by:

\section{The promoters:}

Prof. dr. Markus Pollnau and Prof. dr. Alfred Driessen

The assistant promoter:

Dr. ir. René M. de Ridder 


\section{Contents}

$\begin{array}{ll}\text { Abstract } & 1\end{array}$

Chapter 1 - Introduction 3

1.1 The Raman Effect

1.2 Basic setup for measuring Raman spectra

1.3 The applications

1.3.1 Skin typing

1.3.2 Detection of early dental caries

1.4 Outline of this thesis

Chapter 2 - Integrated technology

2.1 Introduction

2.2 Silicon oxynitride

2.3 Material modeling

2.4 Waveguide design

2.4.1 Waveguides for the skin applications (detection of water and NMF concentrations)

2.4.2 Waveguides for the dental application (detection of early dental caries)

2.4.3 The effective index method

2.5 Summary

Chapter 3 - Signal collection with integrated waveguides

3.1 Introduction

3.2 Integrated waveguide probes for backscattered light collection from weakly scattering media

3.2.1 Mathematical model of integrated waveguide probe

3.2.2 Description of probe

3.2.3 Simulation results 
3.2.4 Experimental results

3.3 Integrated waveguide probes for backscattered light collection from highly scattering media

3.3.1 Monte Carlo modeling of light propagation in turbid media

3.3.2 Optical properties of the scattering medium

\subsubsection{Simulation results}

3.3.4 Experimental results

\subsection{Summary}

Chapter 4 - Confocal integrated light delivery and collection

4.1. Introduction

4.2 Working principle of the arrayed waveguide grating

4.3 An integrated approach to laser delivery and confocal signal detection

4.3.1 Arrayed waveguide grating for the collection of backscattered light from a point source

4.3.2 Arrayed waveguide gratings for laser excitation and confocal signal collection

4.4 Potential of the approach

4.4.1 Imaging properties of the confocal AWG system

4.4.2 Focusing in the vertical direction

4.5 Summary

\section{Chapter 5 - Integrated Raman spectrometers}

5.1 Introduction

5.2 The arrayed waveguide grating for wavelength selection in Raman spectroscopy

5.2.1 The identical bend design

5.2.2 Design and simulation of high order AWGs with the identical-bend layout

5.3 Integrated spectrometer for the skin application

5.3.1 Design and experimental characterization of a broadband AWG for skin NMF concentration detection 
5.4 Integrated spectrometer for the dental application

5.4.1 Design and experimental characterization of the $A W G$ for dental caries detection

5.5 Summary

Chapter 6 - Integrated Raman spectroscopy

6.1 Introduction

6.2 Arrayed-waveguide-grating based Raman spectroscopy

6.2.1 Polarized Raman spectroscopy on cyclohexane

6.2.2 Detection of early dental caries through polarized Raman spectroscopy

6.3 Summary

Chapter 7 - Conclusion and outlook

7.1 Future directions

Appendix A - Design of identical-bend AWG 107

Appendix B - Design of laser suppression filters 111

Appendix C - Design of an AWG based polarization splitter 119

$\begin{array}{ll}\text { List of abbreviations } & 125\end{array}$

$\begin{array}{ll}\text { References } & 127\end{array}$

Samenvatting 133

$\begin{array}{ll}\text { Acknowledgments } & 135\end{array}$

List of publications 139 

$\mathrm{R}$

ecent advances in solid-state lasers and high resolution charge-coupled devices have allowed Raman spectroscopy to emerge as a powerful tool in forensic science, cosmetics, bioscience and nanotechnology. The goal of this research is to contribute even more to the wide spread of this technology by developing low-cost, portable Raman spectrometers, integrated on a chip. In particular the project aims at the fabrication of on-chip devices to be used for the detection of Raman signals from biological samples such as human skin and teeth.

Such spectrometer systems comprise optics for delivering an excitation laser signal to, and collecting the resulting Raman signal from the specimen; a laser line filter; a laser suppression filter; the Raman spectrometer; and, for some applications, polarization splitters. The required light source and photodetectors were not studied in detail within the scope of this thesis.

During the course of our research we opted to use silicon oxynitride as the core material for the integrated waveguide devices due to its low losses and excellent flexibility when used for waveguide design.

In order to face one of the main challenges of our integrated approach, which is signal collection, we initially perform a study on the feasibility of using integrated waveguide probes as alternatives to commonly used fiber probes. We discuss the numerous advantages of integrated probes, in particular comparing their collection efficiency with those of large-core multi-mode and small-core single- and multimode fiber probes.

We also investigate whether channel waveguides are the most efficient devices for collecting backscattered light using integrated optics, and we propose a new integrated optical device which enables focusing of the excitation light and confocal signal collection from a sample under study. The device that we propose makes use of two arrayed waveguide gratings in a confocal arrangement and has a collection efficiency which is an order of magnitude higher than that of a channel waveguide.

Detailed designs of Raman spectrometers are presented for the applications targeted during our research: detection of water and natural moisturizing factor concentrations in the outermost layer of the skin, the stratum corneum, and the detection of early dental caries. We also present a novel arrayed waveguide grating layout on which we base the wavelength selection devices for the skin application. The novel layout makes use of identical bends in all the arrayed waveguides to avoid systematic phase errors arising from the use of different bends in the conventional layouts. 
Finally, we experimentally demonstrate the use of arrayed waveguide gratings to measure polarized Raman spectra from extracted human teeth exhibiting sites with early dental caries. The fabricated device which was designed for the specific application has a high spectral resolution of $0.2 \mathrm{~nm}$ and a free spectral range of 22 $\mathrm{nm}$. The Raman spectra obtained with our device are compared with the measurements obtained using conventional bulk spectrometers, with which they are in excellent agreement. The measured depolarization ratios enable us to clearly distinguish between carious and sound dental regions with the same accuracy obtained using (much larger) conventional spectrometers. 


\section{Chapter 1}

\section{Introduction}

$\mathrm{C}$

handrasekhara Venkata Raman, an Indian physicist, discovered the effect which carries his name (The Raman effect) in 1928. In March and April of that year he published two notes in Nature $\left[\operatorname{Ram} 28^{\mathrm{a}, \mathrm{b}}\right]$ entitled 'A new type of secondary radiation' and 'A change of wave-length in light scattering', followed by another publication in the Indian Journal of Physics [Ram28 $8^{c}$. In his first experiments Raman used as an excitation source a beam of sun light focused on the sample and filtered with a blue-violet filter [Ram28 ${ }^{\mathrm{a}}$. In 1930 he was awarded the Nobel Prize in physics for his discovery.

In those days to acquire a Raman spectrum took very long exposure times, from a few hours to even hundreds of hours [Sin02] depending on the sample (crystals, liquids, vapours, ... etc.) to be measured. The reason is simply that the intensity of the Raman signal is more than a million times lower than that of the excitation beam, making it a difficult task to detect such a signal with the instruments that were available at that time (photographic plates).

Since its discovery, Raman spectroscopy has become a powerful tool for studying molecular structures and crystalline lattices. By using this technique not only is it possible to retrieve information on the type of chemical bonds (qualitative analysis) present in a molecule, but also on the number of these bonds (quantitative analysis), to which the Raman scattering intensity relates linearly. Furthermore, by using polarized Raman spectroscopy (PRS) it is even possible to determine the orientation of the molecules [Tan06]. Other advantages of Raman spectroscopy are that it does not require sample preparation, it is non-destructive (except in case of over-heating from intense excitation radiation), signals can be collected from very small volumes even through glass or polymer cuvette walls, and the measurements are not affected by the presence of water which is a weak Raman scatterer. The latter point makes Raman spectroscopy a suitable technique for the study of biological samples in aqueous solutions [Fer03].

In the first years, Raman spectroscopy experienced a very slow spread due to the lack of stable monochromatic sources and efficient detectors. Only in the past 20 years Raman spectroscopy started to be used in environments other than the chemical laboratories, facilitated by advances in the technology of sources (lasers) and detectors [Han00]. Most recently the development of array detectors has brought many improvements in Raman spectroscopy such as reduced acquisition times, collection of simultaneous Raman spectra, and Raman imaging [Den07]. 
Despite these improvements Raman spectrometers are still very expensive and can only be accessed by a few specialized laboratories. Portable Raman spectrometers have been reported in the literature [Lew93], but their costs are still very high $(\$ 10,000$ on average). In order to make this technique accessible to more laboratories around the world, including poor countries, it is necessary to find a way to reduce the fabrication costs of this equipment. This can be done, for example, by integrating the spectrometers, the detectors, the laser sources and also the laser delivery and signal collection optics on a single chip.

A project named "Raman Pen", aiming at such a cost reduction by realizing small, hand-held, integrated optical spectrometers, is carried out in a cooperation of four knowledge institutes (The University of Twente, Delft University of Technology, Erasmus MC and The National Research Council of Canada) and three companies (River Diagnostics BV, Lionix BV and 2M Engineering Ltd.). It is funded by the Dutch Ministry of Economic Affairs through the "IOP Photonic Devices".

In particular the project aims at the fabrication of on-chip devices that can be used for the detection of Raman signals from biological samples such as human skin and teeth. The target applications are: the detection of water concentration in human skin, the detection of the natural moisturizing factor (NMF) of human skin and the detection of early dental caries. They have been chosen for their diversity in terms of requirements, giving more generality to the project results, but also in part for their high market potential.

In this Chapter we describe the requirements of each of these applications. But first, we give a brief and basic introduction to the Raman effect for the reader to familiarize with before going into the details of device design given in the following chapters of this thesis. We also show a basic setup for measuring Raman spectra and explain the function of its components.

In the final Section of this Chapter we provide the outline of the thesis.

\subsection{The Raman effect}

The Raman effect is a phenomenon in which the wavelength of light is changed by interaction with matter. As light propagates in a medium it interacts with matter in a variety of ways. In simplified terms we can think of three types of interaction: absorption, emission and scattering. Scattering processes involve the absorption of a photon and the simultaneous emission of another photon [Ru09]. These can be separated into two groups: elastic scattering processes in which the emitted photon has the same energy and frequency as the incident photon (Rayleigh scattering), and inelastic scattering processes were the two photons have different energies and frequencies. The Raman effect is a type of inelastic scattering. 
The Raman effect can be described in terms of the classical electromagnetic theory, according to which the electrons and nuclei of molecules exposed to an electric field $\mathbf{E}$ are set into motion following Coulomb's law, and a dipole moment $\mathbf{p}$ is induced in the molecule:

$$
\mathbf{p}=\boldsymbol{\alpha} \cdot \mathbf{E},
$$

where $\alpha$ is the polarizability of the molecule: it is a measure of the ease with which the electrons of the molecule can be displaced under the action of an electric field. The polarizability $\alpha$ is a second-rank tensor whose components have units of $\mathrm{CV}^{-}$ ${ }^{1} \mathrm{~m}^{2}$. This implies that, in general, each component of the induced dipole moment is a linear combination of all three components of the electric field. In (1.1) we neglected the presence of nonlinear terms that arise when strong electric fields are applied to the molecule [Lon77].

A molecule is internally subject to electronic, vibrational and rotational transitions which cause the polarizability of the molecule to change. For this reason we can express the polarizability $\boldsymbol{\alpha}$ as the sum of a static term $\boldsymbol{\alpha}_{0}$ and an oscillating term $\boldsymbol{\alpha}_{1}$ that oscillates with the frequency $\omega_{\nu}$ of a molecular transition:

$$
\boldsymbol{\alpha}=\boldsymbol{\alpha}_{0}+\boldsymbol{\alpha}_{1} \cos \omega_{v} t
$$

Let the amplitude and frequency of the incident electric field be $\mathbf{E}_{\mathrm{i}}$ and $\omega_{i}$, respectively. In this case the induced dipole moment can be expressed as:

$$
\mathbf{p}=\boldsymbol{\alpha} \cdot \mathbf{E}_{i} \cos \omega_{i} t=\left(\boldsymbol{\alpha}_{0}+\boldsymbol{\alpha}_{1} \cos \omega_{v} t\right) \cdot \mathbf{E}_{i} \cos \omega_{i} t .
$$

The previous equation, by the use of a simple trigonometric identity, can be written as:

$$
\mathbf{p}=\boldsymbol{\alpha}_{0} \cdot \mathbf{E}_{i} \cos \omega_{i} t+\frac{1}{2} \boldsymbol{\alpha}_{1} \cdot \mathbf{E}_{i} \cos \left(\omega_{i}-\omega_{v}\right) t+\frac{1}{2} \boldsymbol{\alpha}_{1} \cdot \mathbf{E}_{i} \cos \left(\omega_{i}+\omega_{v}\right) t
$$

As can be seen from (1.4) the induced dipole moment is composed of three terms: the first term corresponds to Rayleigh scattering at the same frequency as the incident light, while the second and third terms correspond to Stokes (frequency difference) and anti-Stokes (frequency sum) Raman scattering. The classical derivation is just an approximation since in normal conditions the Stokes and antiStokes scattering intensities are not the same. The intensity of anti-Stokes scattering is much smaller than that of Stokes scattering. This can be understood with the help of the energy level diagram shown in Fig. 1.1, from which we see that for the antiStokes Raman emission to occur the molecules must be at an excited level. The number of molecules in the excited level follows the Boltzmann distribution and is usually smaller than that of the molecules in the ground state [Ber05]. 


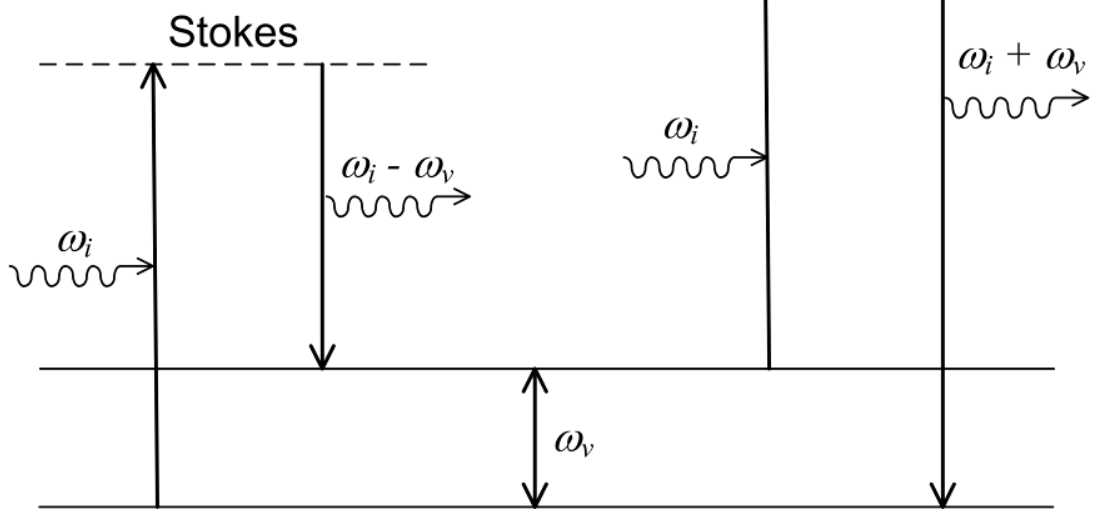

Fig. 1.1 - Energy-level diagram of Stokes and anti-Stokes Raman scattering.

A more detailed study of Raman scattering is beyond the scope of this thesis.

In this Thesis, when representing Raman spectra we will use, as conventionally done, the units of relative inverse centimetres (or reciprocal centimetres). The $\mathrm{cm}^{-1}$ unit is proportional to the frequency and photon energy, and indicates how many wavelengths (of light) are present in a length of $1 \mathrm{~cm}$. Even though, in the International System of Units (SI) a reciprocal length is expressed in units of inverse meters $\left(\mathrm{m}^{-1}\right)$, for historical reasons and also for convenience, the units of $\left(\mathrm{cm}^{-1}\right)$ are still used in spectroscopy.

In Raman spectroscopy contrary to other types of spectroscopy, as for example Fourier transform infrared (FTIR) spectroscopy, it is a common practice not simply to represent the measured spectra in terms of wavenumbers, but as wavenumber shifts relative to the wavenumber of the excitation source. For example if we use an excitation source having a wavelength of $\lambda_{\mathrm{e}}=830 \mathrm{~nm}$, and one of our Raman peaks falls at a wavelength of $\lambda_{\mathrm{R}}=901.78 \mathrm{~nm}$, then using the convention of Raman spectroscopy we would represent the peak centred at a wavenumber $v_{\mathrm{R}}$ given by:

$$
v_{R}=\left(\frac{1}{\lambda_{e}}-\frac{1}{\lambda_{R}}\right) \cdot 1 \times 10^{7}=959 \mathrm{~cm}^{-1}
$$

\subsection{Basic setup for measuring Raman spectra}

A Stokes Raman spectrum can be measured using the setup shown in Fig. 1.2. Light from an excitation laser is propagated through a laser line filter to suppress any unwanted spectral components of the source. The filtered light is reflected from a dichroic mirror designed to present a high reflectivity at the laser wavelength and high transparency for longer wavelengths. The light is then focused on the sample 6 
under study using a lens. The backscattered (Rayleigh and Raman) and backreflected light from the sample is collimated by the same lens and propagates again towards the dichroic mirror. The mirror reflects most ( 98\%) of the light at the laser wavelength and transmits the Raman signal. The small portion of laser light that passes through the dichroic mirror can between $10^{6}$ and $10^{8}$ times more intense than the Raman signal. For this reason an edge filter is used to suppress the residual laser light. The filtered signal is focused onto a diffraction grating which separates the different spectral components that are focused onto different locations of a linear CCD array.

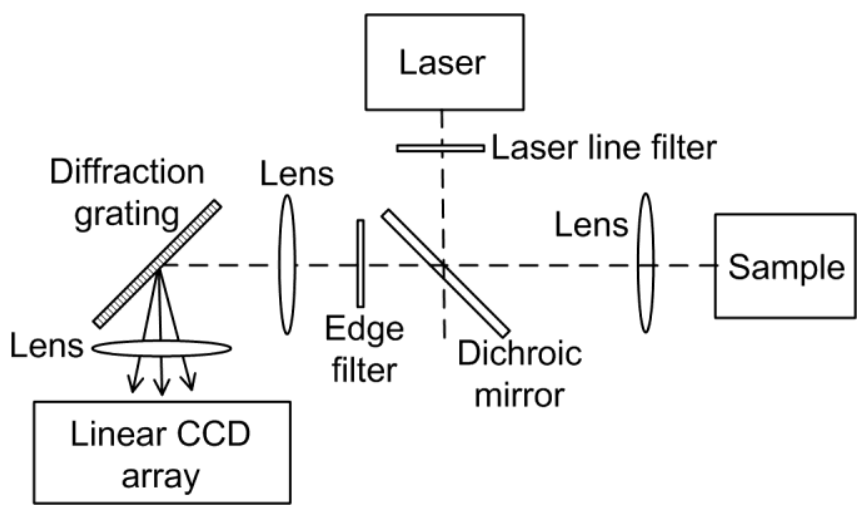

Fig. 1.2 - Basic setup for measuring Raman spectra.

In the Raman Pen project we aim at integrating on a chip the main components shown in Fig. 1.2: the grating, the edge filter (or laser suppression filter) and the laser delivery and signal collection optics. The main challenge is to find suitable integrated optical components that have the same functionality and comparable performance as the bulky parts.

\subsection{The applications}

In this Section we introduce the three applications that we have targeted in our project. These can be divided into two categories. The first category is related to Raman spectroscopy of the skin. Into this category fall two applications, the main goal of which is skin typing for the cosmetic industry. The second category is more in the biomedical field and relates to Raman spectroscopy of teeth. Here the goal is the detection of early dental caries through polarized Raman spectroscopy.

\subsubsection{Skin typing}

Currently River Diagnostics (a partner in the Raman Pen project) has developed and commercialized an in vivo skin composition analyzer (SCA model 3510) which enables a detailed quantitative analysis of the skin's molecular composition, with high spatial resolution. One of the applications of the SCA is to enable the personal care and cosmetic industries to study penetration of topically applied compounds 
into the skin in relation to the skin's properties in terms of water concentration, lipid/protein ratios and natural moisturizing factor (NMF) concentration. Currently the technology is used in test centers where efficacy tests of personal care products are being carried out in panel studies.

The Raman Pen project aims at the fabrication of a low-cost and portable version of this device to be used inside stores to enable customers to have their skin type determined prior to purchasing a product. The water concentration and lipid/protein ratio can be determined by means of spectra in the $\mathrm{CH} / \mathrm{OH}$-stretching region (between $2750 \mathrm{~cm}^{-1}$ and $3800 \mathrm{~cm}^{-1}$ ) of the Raman spectrum of the stratum corneum, whilst the NMF concentration can be determined in the so called fingerprint region (400-1800 $\mathrm{cm}^{-1}$ ) of the Raman spectrum of the stratum corneum [Cas03].

In Fig. 1.3 a Raman spectrum of the skin in the region between $2500 \mathrm{~cm}^{-1}$ and $4000 \mathrm{~cm}^{-1}$ (which includes the $\mathrm{CH} / \mathrm{OH}$-stretching region) is shown for different values of water concentration. In this spectral region five different bands are indicated: bands $\mathrm{A}$ and $\mathrm{B}$ define the background, $\mathrm{L}$ is the lipid band, $\mathrm{P}$ is the protein band and $\mathrm{W}$ is the water band. As the water concentration increases the signal in the water band increases while the lipid/protein ratio does not change significantly.

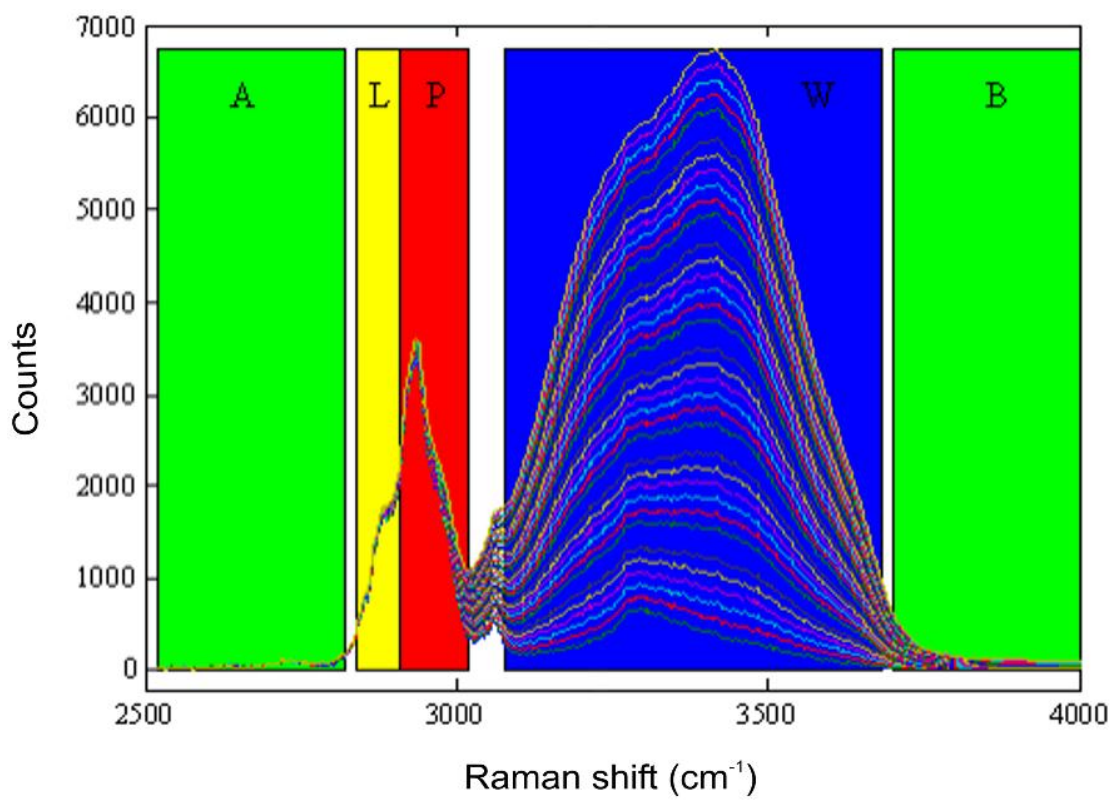

Fig. 1.3 - Spectrum of the stratum corneum in the $\mathrm{CH} / \mathrm{OH}$-stretching spectral region for different values of water concentration [Cas01]. The bands A and B are used for detecting the background. $\mathrm{L}$ is the lipid band, $\mathrm{P}$ the protein band and $\mathrm{W}$ is the water band. 
In Table 1.1 we give the central positions and widths of the five bands expressed in wavenumber units as well as in nanometers at an excitation wavelength of 671 $\mathrm{nm}$.

Table 1.1 - Bands of interest for water concentration and lipid/protein ratio detection

\begin{tabular}{ccccc}
\hline Bands & $\begin{array}{c}\text { Center } \\
\left(\mathbf{c m}^{-\mathbf{1}}\right)\end{array}$ & $\begin{array}{c}\text { Width } \\
\left(\mathbf{c m}^{-\mathbf{1}}\right)\end{array}$ & $\begin{array}{c}\text { Center } \\
(\mathbf{n m})\end{array}$ & $\begin{array}{c}\text { Width } \\
(\mathbf{n m})\end{array}$ \\
\hline $\mathbf{A}$ & 2670 & 300 & 817.45 & 20.05 \\
$\mathbf{L}$ & 2875 & 70 & 831.38 & 4.83 \\
$\mathbf{P}$ & 2965 & 110 & 837.65 & 7.72 \\
$\mathbf{W}$ & 3380 & 600 & 867.82 & 45.22 \\
$\mathbf{B}$ & 3850 & 300 & 904.72 & 24.56 \\
\hline
\end{tabular}

In Fig. 1.4 we show an example of a Raman spectrum of the skin (blue line) in the fingerprint region between $400 \mathrm{~cm}^{-1}$ and $1800 \mathrm{~cm}^{-1}$. From this spectrum we want to determine the concentration of NMF. The NMF is a water-soluble mixture of free amino acids, their derivatives and specific salts. It is produced in the lower part of the stratum corneum [Cas01] and can be extracted using different techniques [Koy83, Syl10]. In Fig. 1.4 we also show the spectrum of extracted NMF for completeness.

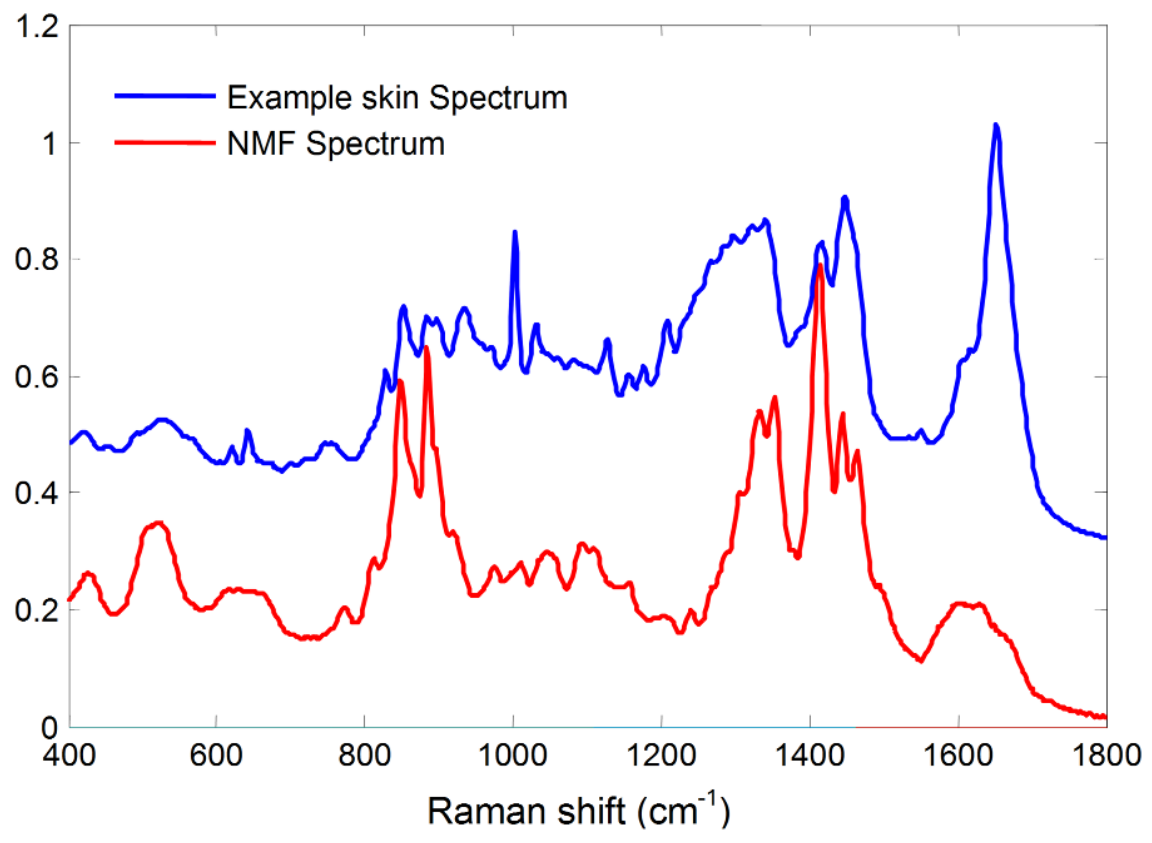

Fig. 1.4 - Raman spectra of the skin and the NMF in the fingerprint spectral region. 
The concentration of NMF in the skin may vary and can be determined by fitting the measured spectrum of the skin with a "model set" of normalized NMF spectra [Cas01]. In Table 1.2 we indicate the relevant spectral bands from which information for skin typing can be derived. The central positions and widths of the four bands are indicated in wavenumber units as well as in nanometers with respect to an excitation wavelength of $785 \mathrm{~nm}$.

Table 1.2 - Band of interest for the natural moisturizing factor (NMF) concentration detection

\begin{tabular}{ccccc}
\hline Bands & $\begin{array}{c}\text { Center } \\
\left(\mathbf{c m}^{-1}\right)\end{array}$ & $\begin{array}{c}\text { Width } \\
\left(\mathbf{c m}^{-1}\right)\end{array}$ & $\begin{array}{c}\text { Center } \\
(\mathbf{n m})\end{array}$ & $\begin{array}{c}\text { Width } \\
(\mathbf{n m})\end{array}$ \\
\hline $\mathbf{A}$ & 805 & 42 & 837.96 & 2.95 \\
$\mathbf{B}$ & 1245 & 38 & 870.03 & 2.88 \\
$\mathbf{C}$ & 1385 & 38 & 881.54 & 2.95 \\
$\mathbf{D}$ & 1608 & 80 & 898.42 & 6.46 \\
\hline
\end{tabular}

The spectra in Figs. 1.3 and 1.4 and the band information in Tables 1.1 and 1.2 were provided by our project partners (Erasmus MC and River Diagnostics).

\subsubsection{Detection of early dental caries}

The detection of dental caries at early stages allows dentists to use non-surgical treatment approaches (fluoride treatment, sealants and antimicrobials) rather than more invasive restorative methods such as drilling and filling. However, detecting and monitoring these early lesions is difficult with currently available diagnostic tools (dental explorer and radiographs) [Bad01, Ism04, Hor04].

Research carried out by the Institute for Biodiagnostics of the National Research Council in Canada (NRC-IBD) together with other clinical collaborators from Canadian universities (Manitoba and Dalhousie) demonstrates that it is possible to adopt optical coherence tomography (OCT) and polarized Raman spectroscopy (PRS) to address the problem of early dental caries detection [Ko06, Ko08, Cho10]. In particular, it has been shown that analyses with PRS indicate statistically significant differences in the depolarization ratio of the Raman phosphate $\left(\mathrm{PO}_{4}{ }^{3-}\right)$ symmetric vibration at $\sim 959 \mathrm{~cm}^{-1}$ arising from tooth hydroxyapatite (see Fig. 1.5). The depolarization ratio is higher in carious regions than in sound regions and the difference is attributed to alterations in the enamel rod morphology and orientation of the enamel crystallites resulting from acid-induced demineralization. 


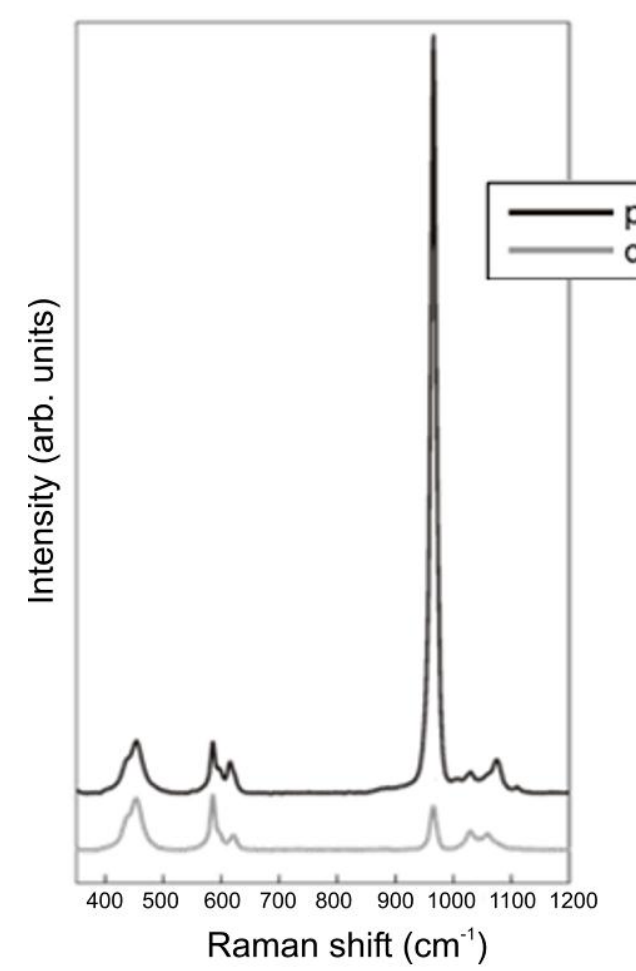

(a)

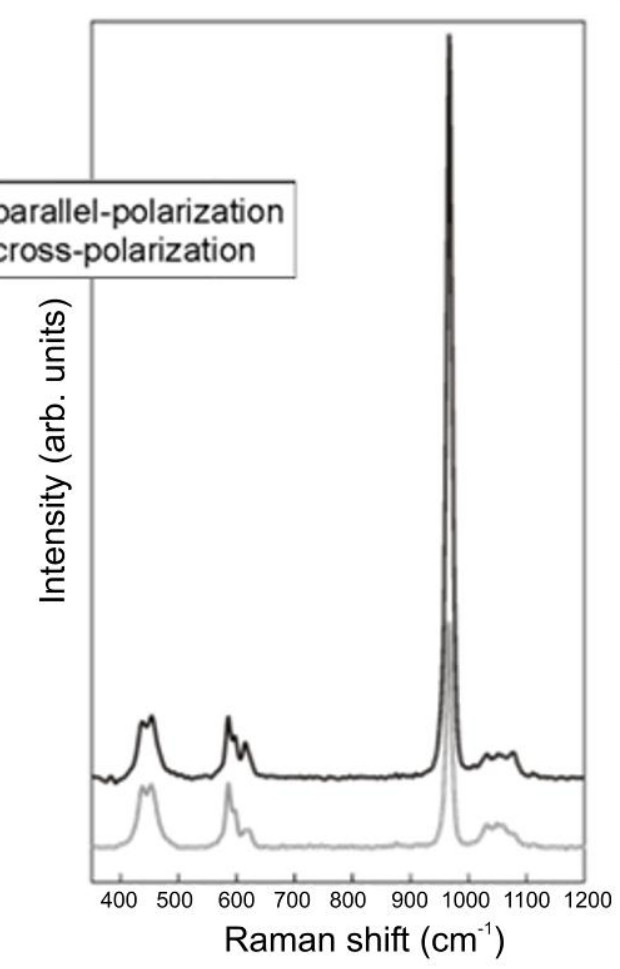

(b)

Fig. 1.5 - Raman spectra of A) healthy enamel, and B) carious enamel, for both parallel and cross polarizations.

These observations support the development of new clinical tools based on Raman spectroscopy for the detection and monitoring of the processes of tooth demineralization and remineralization.

The excitation wavelength chosen for this application is $830 \mathrm{~nm}$ since it generates a lower fluorescence background in the case of stained teeth as compared with shorter wavelengths such as $785 \mathrm{~nm}$. In principle, going to even longer wavelengths than $830 \mathrm{~nm}$ can reduce the fluorescence, but this is not a common practice since it reduces the efficiency of Raman scattering, which is inversely proportional to the fourth power of wavelength.

Studies carried out on the characterization of early dental caries [Ko05] showed that upon demineralization of the tooth enamel the hydroxyapatite peak does not shift from its position, however shifts of about 2 to $5 \mathrm{~cm}^{-1}$ were observed upon remineralization [personal communication from L.-P. Choo-Smith]. In order to resolve these subtle peak shifts the filter device must have a resolution of around 0.2 $\mathrm{nm}$. In addition the device must have a sufficiently large free-spectral range (FSR) (from $820 \mathrm{~cm}^{-1}$ to $1100 \mathrm{~cm}^{-1}$ ) in order to fully resolve the hydroxyapatite peak and 
enable the evaluation of any baseline influence (for example due to staining). In wavelength units this results in an FSR of $22 \mathrm{~nm}$ in the range between $890 \mathrm{~nm}$ and $912 \mathrm{~nm}$.

The filter specifications for the detection of the hydroxyapatite Raman peak are given in Table 1.3. The wavelength values are given assuming an excitation wavelength of $830 \mathrm{~nm}$.

Table 1.3 - Filter specifications for the detection of the hydroxyapatite Raman peak

\begin{tabular}{lcc}
\hline & $\mathbf{c m}^{-\mathbf{1}}$ & $\mathbf{n m}$ \\
\hline Central wavelength & 959 & 901.78 \\
Resolution & $2-5$ & 0.2 \\
Wavelength range & $820-1100$ & $890-912$ \\
FSR & 280 & 22 \\
\hline
\end{tabular}

\subsection{Outline of this thesis}

This thesis describes the design, fabrication and characterization of integrated optical light delivery and signal collection devices, and spectrometers for three specific applications that involve Raman spectroscopy of biological tissues such as skin and teeth. These applications have been the subject of this first introductory Chapter.

In Chapter 2 we introduce the technology used to fabricate the integrated devices proposed throughout the thesis. We refer to the silicon oxynitride waveguide technology which is well established in the Integrated Optical MicroSystems Group of the University of Twente where this research has been conducted. In Chapter 2 we also discuss the material model used for the waveguide design.

Chapter 3 focuses on signal collection through integrated optical waveguides. Here we show the advantages that integrated optical waveguide probes can have with respect to fiber optical probes. We also present a mathematical model (validated by comparison with the experiment) for estimating the collection efficiency of integrated waveguide probes with arbitrary geometrical cross-sections. The model is valid for the particular case of weakly scattering media. For the case of highly scattering media we also present our results based on Monte-Carlo simulations.

In Chapter 4 we propose a novel method for the delivery of excitation light to the sample under study and the confocal collection of backscattered light. The method makes use of a confocal arrangement of arrayed waveguide gratings, of which the working principle is described in the same Chapter. 
Chapter 5 presents the descriptions of the Raman spectrometers for the skin and dental applications. The designs, together with the results of the experimental characterization of the different constituting blocks are presented in great detail.

Our Raman experiments performed using the integrated devices for the dental applications are presented in Chapter 6 . We prove that with our devices not only it is possible to detect Raman signals from test samples such as cyclohexane, but also from biological samples, in this specific case teeth, for which we show promising results of PRS measurements. 


\section{Chapter 2}

\section{Integrated technology}

\subsection{Introduction}

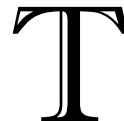

he Chapter starts with an introduction on silicon oxynitride as the material of choice for the fabrication of integrated optical devices in this thesis. We then

describe the standard processing steps adopted for the fabrication of optical waveguides in silicon oxynitride technology. All the mentioned steps were performed in the clean room facilities of the MESA+ Institute for Nanotechnology by the technicians and researchers of the Integrated Optical MicroSystems group of the University of Twente.

In Section 2.3 we introduce the mathematical models that we adopted for the waveguide cladding and core materials. These models are based on the Sellmeier dispersion formula. Finally in Section 2.4 we present details on the waveguide design and optimization of the fiber-to-chip coupling. We also discuss the effective index method used to approximate the $3 \mathrm{D}$ waveguide structure with a $2 \mathrm{D}$ equivalent in order to perform reliable bi-dimensional beam-propagation simulations.

\subsection{Silicon oxynitride}

For the realization of all integrated optical devices discussed throughout this thesis we used silicon oxynitride $(\mathrm{SiON})$. The choice for $\mathrm{SiON}$-based waveguides was motivated by its excellent optical properties as well as the availability of reliable fabrication tools. Since low optical propagation loss is one of the primary requirements for integrated optical devices, the transparency of the waveguide material is of importance. SiON is highly transparent over a wide wavelength range from $0.2 \mu \mathrm{m}$ to $2.5 \mu \mathrm{m}$. Planar and channel waveguides with optical losses as low as $0.08 \mathrm{~dB} / \mathrm{cm}$ at $1560 \mathrm{~nm}$ have been reported [Ger00]. Of particular interest to the Raman application is the absence of any strong luminescence or absorption in the spectral region between $800 \mathrm{~nm}$ and $900 \mathrm{~nm}$ [Wör99 , Mor99, Pri99] where the usually weak Raman signals are located. In addition, for the realization of complex functional integrated optical devices access to reliable technology enabling the reproducible fabrication of uniform waveguides is required. Our SiON technology is based on well-developed, standard deposition and etching techniques like CVD and RIE. Films with good uniformity of thickness (between $0.8 \%$ and $3 \%$ ) and refractive index (between $2 \times 10^{-4}$ and $6 \times 10^{-4}$ ) over the entire wafer area can be deposited [Rid98]. The run-to-run reproducibility of the layer thickness and refractive index is $2 \%$ and $\sim 6 \times 10^{-4}$, respectively [Rid98].

Furthermore the refractive index of $\mathrm{SiON}$ can be tuned over a wide range, from 1.46 to 2.0 , by changing the ratio between the oxygen and nitrogen content in the 
material [Pet91]. This is of interest mainly for the waveguide design, where the wide tuning range of the optical parameters gives a large flexibility to the designer for tailoring specific waveguide properties. Last but not least, the fact that standard silicon substrates can be used adds to the attractiveness of this material, since eventually the integration of optical chips with detectors and other electronic circuits is enabled [Wun92].

SiON can be deposited using either low-pressure chemical vapour deposition (LPCVD) or plasma-enhanced chemical vapour deposition (PECVD). Both techniques involve the reaction between gas precursors containing silicon, oxygen, and nitrogen. The energy source for the reaction is provided by temperature in case of LPCVD and plasma in case of PECVD. In our cleanroom the machines used for the two processes are the Tempress hot-wall quartz-tube reactor for LPCVD and the Oxford Plasmalab 80 machine for PECVD. In the LPCVD process the gasses introduced in the reactor are $\mathrm{SiH}_{2} \mathrm{Cl}_{2}, \mathrm{NH}_{3}$, and $\mathrm{O}_{2}$. The gasses and substrate are heated to temperatures between 750 and $900^{\circ} \mathrm{C}$ for the reaction to take place [Alb96, Wör99a ]. LPCVD is well suited for depositing layers with refractive indices between 1.7 and 2, for which the deposition rate is in the range between 2 and $10 \mathrm{~nm} / \mathrm{min}$ [Wör98]. For layers with lower refractive index the deposition rate becomes $<<1$ $\mathrm{nm} / \mathrm{min}$, leading to unacceptably long deposition times because of accordingly higher cleanroom costs. On the other hand, in the PECVD process the reaction gasses are $\mathrm{N}_{2} \mathrm{O}$ and $\mathrm{SiH}_{4}$ diluted in $\mathrm{N}_{2}$. The layers are deposited at $60 \mathrm{~W}$ plasma power, $650 \mathrm{mTorr}$ pressure and $300^{\circ} \mathrm{C}$ temperature [Wör99a]. In particular, with $\mathrm{SiH}_{4}$ being more reactive than $\mathrm{SiH}_{2} \mathrm{Cl}_{2}$ (used in LPCVD), the deposition rates in case of PECVD are much higher (> $20 \mathrm{~nm} / \mathrm{min}$ ) making the process better suited for the deposition of thick layers with low refractive index [Wör98].

As will be discussed in Section 2.4 (on waveguide design), for our devices we need a refractive index of $\mathrm{SiON}$ around 1.509 at $830 \mathrm{~nm}$, as well as layer thicknesses above $500 \mathrm{~nm}$. For this reason our choice inevitably falls on the use of PECVD deposition. Films deposited with this technique present a strong absorption centred at a wavelength of $1510 \mathrm{~nm}$. The losses at this wavelength have been measured to be $\sim 10 \mathrm{~dB} / \mathrm{cm}$ for a refractive index value of 1.68 [Wör99 $]$ ]. These losses are due to overtones of the resonant frequency of hydrogen bonds ( $\mathrm{Si}-\mathrm{H}$ and $\mathrm{N}-\mathrm{H}$ ) and can be significantly reduced (to $\sim 0.6 \mathrm{~dB} / \mathrm{cm}$ ) by annealing the wafer at high temperatures [Alb95, Wör99a, Roe02].

The waveguide structures were fabricated as shown schematically in Fig. 2.1. A SiON film was deposited by PECVD on a thermally oxidized $\langle 100\rangle$ silicon wafer with a diameter of $100 \mathrm{~mm}$, having an oxide thickness of $8 \mu \mathrm{m}$. Details on the deposition parameters are given in Table 2.1, while typical results on the refractive index and thickness obtained after deposition will be given in Section 2.4 on waveguide design. After deposition the layer is annealed around $1100^{\circ} \mathrm{C}$ for 3 hours [Hus01]. 


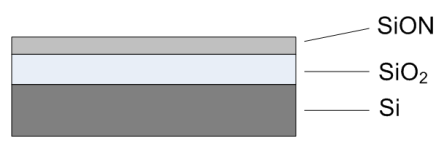

(a)

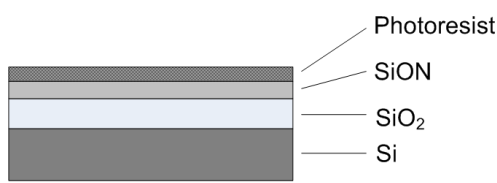

(b)

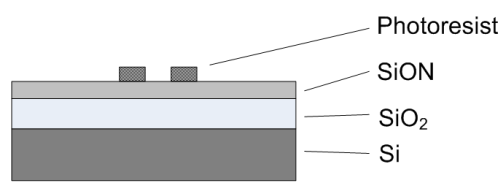

(c)

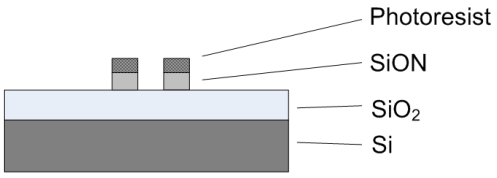

(d)

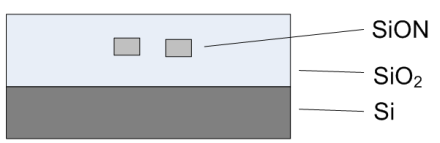

(e)

Fig. 2.1 - Steps of the fabrication process of waveguide structures. (a) SiON deposition, (b) spinning of positive photoresist, (c) development of photoresist, (d) RIE etching of $\mathrm{SiON}$, and removal of photoresist (e) top cladding deposition.

Table 2.1 - Parameters for PECVD SiON deposition

\begin{tabular}{cc}
\hline Plasma power $(\mathbf{W})$ & 60 \\
Plasma frequency $(\mathbf{k H z})$ & 187.5 \\
Chamber pressure $(\mathbf{m T o r r})$ & 650 \\
Substrate temperature $\left({ }^{\circ} \mathbf{C}\right)$ & 300 \\
$\mathrm{SiH}_{4} / \mathbf{N}_{\mathbf{2}}$ flow $(\mathbf{s c c m})$ & 600 \\
$\mathbf{N}_{\mathbf{2}} \mathrm{O}$ flow $(\mathbf{s c c m})$ & (Variable for \\
& index tuning) \\
\hline
\end{tabular}

The wafer was then covered with a thin positive photoresist layer (OIR 907/12) of $\sim 600 \mathrm{~nm}$ thickness, and an e-beam written mask (chromium on quartz with minimum resolvable feature size of $0.7 \mu \mathrm{m}$, and a defect density of 0.64 defects/inch ${ }^{2}$ with maximum defect size of $2 \mu \mathrm{m}$ ) was used to transfer the waveguide pattern to the resist with a high side-wall quality (see Fig. 2.2). The use of a thin resist layer enables one to open gaps as small as $0.7 \mu \mathrm{m}$ [Sen11] between the waveguides. The waveguide structures were then etched into the SiON using reactive ion etching (RIE) on the Plasmatherm 790 machine. The etch parameters are given Table 2.2. The resulting pattern on SiON is shown in Fig. 2.3. 


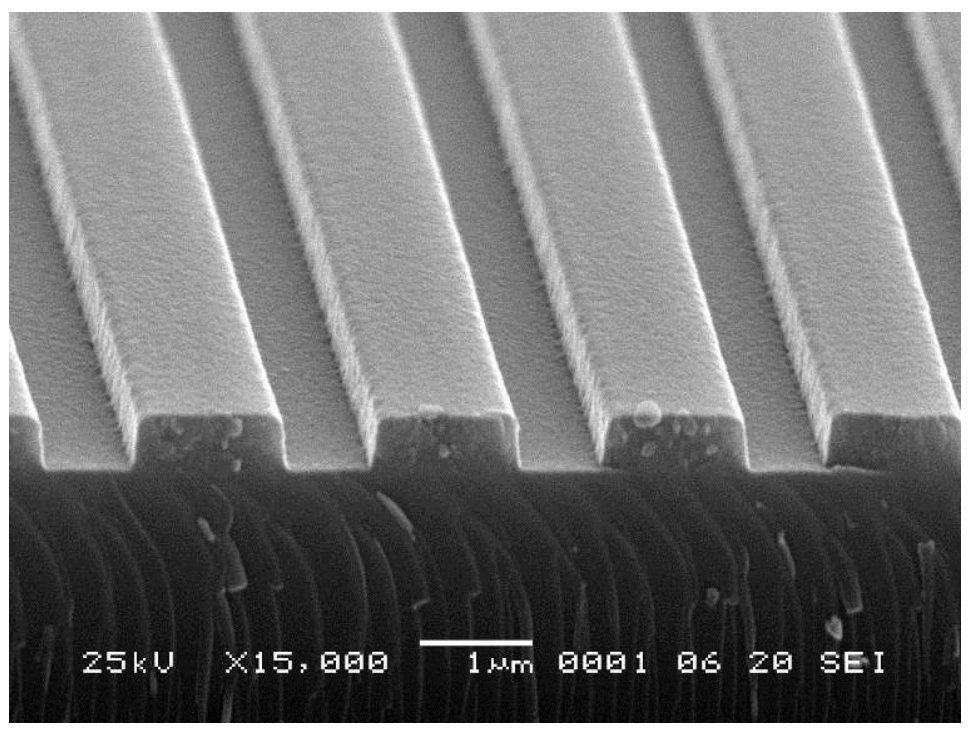

Fig. 2.2 - SEM image of the patterned photoresist obtained after development in positive photoresist developer.

Table 2.2 - Parameters for reactive ion etching of SiON Plasma power (W)

Chamber pressure (mTorr) 28

Substrate temperature $\left({ }^{\circ} \mathrm{C}\right) \quad 20$

$\mathrm{O}_{2}$ flow (sccm) 3

$\mathrm{CHF}_{3}$ flow (sccm) 100

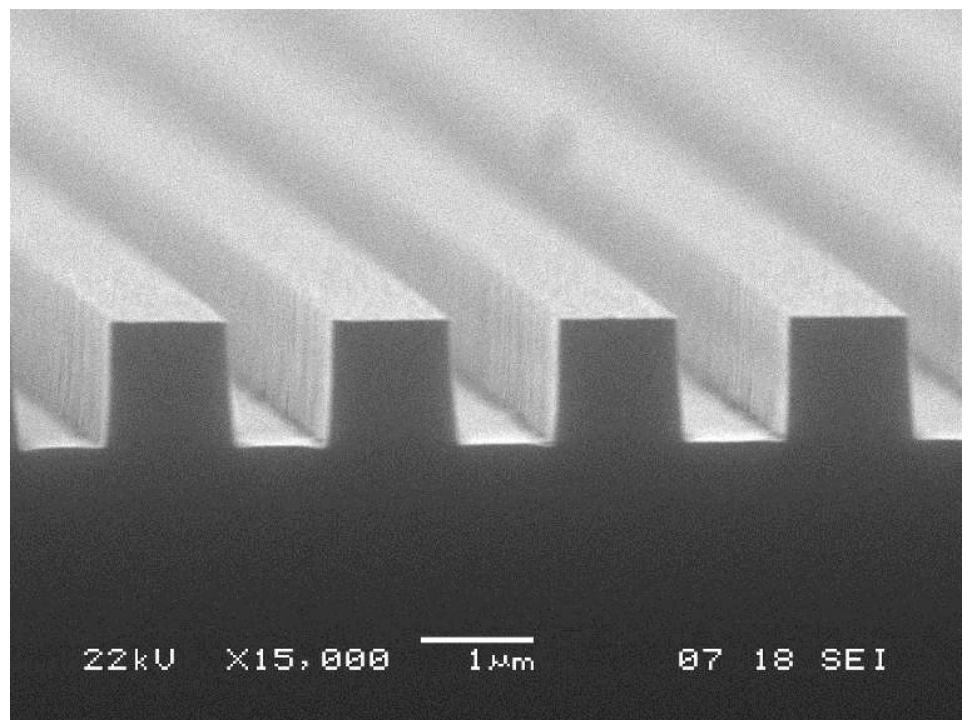

Fig. 2.3 - SEM image showing the waveguide structures transferred to the SiON layer after reactive ion etching (RIE). 
The top cladding was deposited in two steps. First a $1 \mu \mathrm{m}$ layer of $\mathrm{SiO}_{2}$ was deposited using LPCVD from tetra-ethyl-ortho-silicate (TEOS) at a temperature between 710 and $740^{\circ} \mathrm{C}$, and annealed at $1100^{\circ} \mathrm{C}$ for three hours. Then a $3 \mu \mathrm{m}$ layer of $\mathrm{SiO}_{2}$ was deposited using PECVD at a temperature of $300^{\circ} \mathrm{C}$ and low frequency $\mathrm{RF}$ power of $60 \mathrm{~W}$. The layer stack was again annealed for three hours at $1150^{\circ} \mathrm{C}$. The parameters for the top cladding deposition are shown in Table 2.3, while the waveguide structure after cladding deposition is shown in Fig. 2.4. We observe that the waveguide walls are not perfectly vertical. However, the angle $\vartheta$ between the waveguide wall and the horizontal plane is between $88.5^{\circ}$ and $89^{\circ}$, causing a negligible variation in the waveguide width $(\sim 0.03 \mu \mathrm{m})$ along the vertical direction. Since this variation is well below the fabrication tolerances $( \pm 0.1 \mu \mathrm{m})$ and also much smaller than the waveguide width, we did not account for it in our simulations.

Table 2.3 - Parameters for top cladding deposition

\begin{tabular}{ccc}
\hline & $\mathbf{S i O}_{2}(\mathbf{L P C V D})$ & $\mathbf{S i O}_{\mathbf{2}}(\mathbf{P E C V D})$ \\
\hline Plasma power $(\mathbf{W})$ & - & 60 \\
Chamber pressure $(\mathbf{m T o r r})$ & 400 & 650 \\
Substrate temperature $\left({ }^{\circ} \mathbf{C}\right)$ & $710-740$ & 300 \\
TEOS flow $(\mathbf{s c c m})$ & 40 & - \\
$\mathbf{N}_{\mathbf{2}}$ flow $(\mathbf{s c c m})$ & 30 & - \\
$\mathbf{N}_{\mathbf{2}} \mathbf{O}$ flow $(\mathbf{s c c m})$ & - & 710 \\
$\mathbf{2 \%} \mathbf{S i H}_{4} / \mathbf{N}_{\mathbf{2}}(\mathbf{s c c m})$ & - & 200 \\
\hline
\end{tabular}

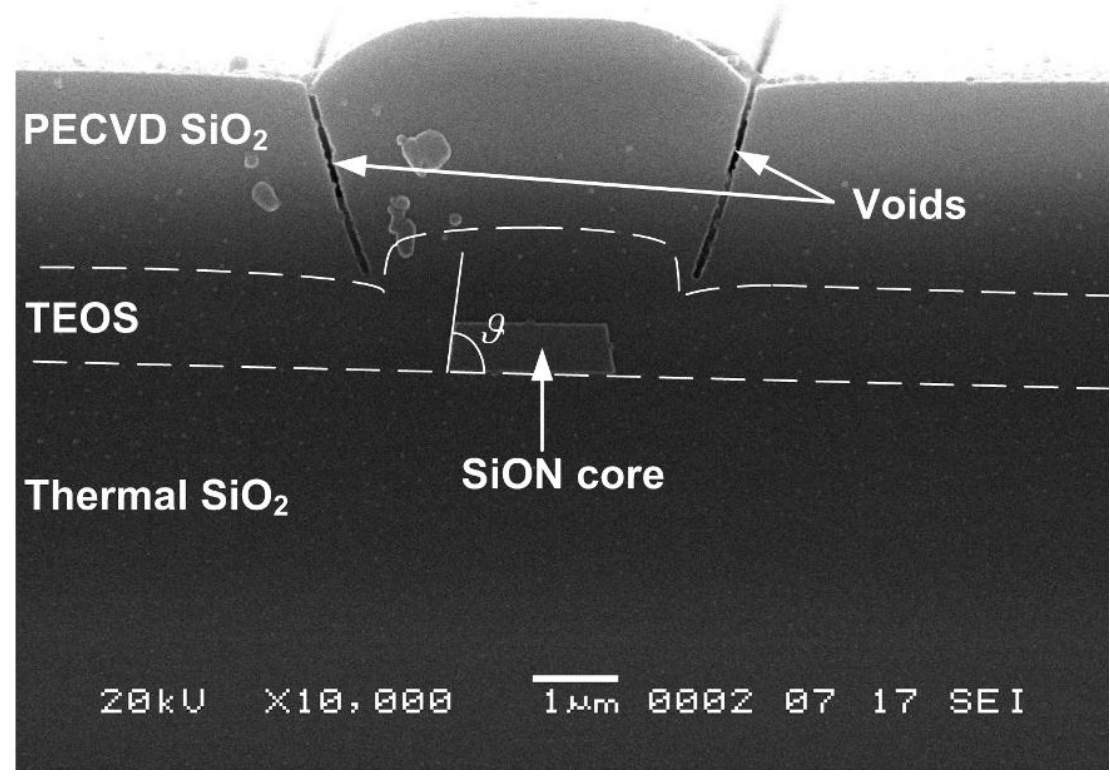

Fig. 2.4 - SEM image showing the waveguide structure after deposition of the top cladding. 
We also observe that the PECVD $\mathrm{SiO}_{2}$ layer presents voids at both sides of the channel waveguide. The voids are a common problem encountered during cladding deposition and are due to a shadowing effect from the oxide deposited on top of the core [Dai05]. The voids are not present in the LPCVD $\mathrm{SiO}_{2}$ layer deposited from TEOS which has a much better step coverage [Is193]. In our simulations the voids are not taken into account since they are sufficiently far away from the modal field propagating in the waveguide which has a diameter of $\sim 3 \mu \mathrm{m}$.

\subsection{Material modeling}

The $\mathrm{SiO}_{2}$ model used for simulating the waveguide structures was constructed from refractive index measurements on thermal oxide performed in our group. The measurements were performed using a Metricon prism coupler (model 2010) at four different wavelengths, and for both transverse-electric (TE) and transverse-magnetic (TM) polarizations on two distinct layers of thermal $\mathrm{SiO}_{2}$ on a silicon substrate. The refractive index measurement accuracy in the Metricon setup is $\sim 1 \times 10^{-3}$. The results are shown in Table 2.4, from which we observe that the birefringence is almost constant $\left(n_{\mathrm{TM}}-n_{\mathrm{TE}} \cong 1 \times 10^{-3}\right)$ for all wavelength values.

Table 2.4 - Refractive index measurements of thermal $\mathrm{SiO}_{2}$

\begin{tabular}{ccccc}
\hline Wavelength $(\mathbf{n m})$ & $\begin{array}{c}\text { TE } \\
\text { (sample 1) }\end{array}$ & $\begin{array}{c}\text { TM } \\
\text { (sample 1) }\end{array}$ & $\begin{array}{c}\text { TE } \\
\text { (sample 2) }\end{array}$ & $\begin{array}{c}\text { TM } \\
\text { (sample 2) }\end{array}$ \\
\hline $\mathbf{6 3 2 . 8}$ & 1.4581 & 1.4594 & 1.4580 & 1.4591 \\
$\mathbf{8 3 0}$ & 1.4541 & 1.4552 & 1.4542 & 1.4552 \\
$\mathbf{1 3 0 0}$ & 1.4482 & 1.4492 & 1.4485 & 1.4496 \\
$\mathbf{1 5 5 0}$ & 1.4453 & 1.4463 & 1.4451 & 1.4461 \\
\hline
\end{tabular}

Furthermore, in our model we consider the refractive index of the top cladding, which is a combination of TEOS ( $1 \mu \mathrm{m}$ thickness) and PEVCD $\mathrm{SiO}_{2}(\sim 3 \mu \mathrm{m}$ thickness), the same as that of thermal oxide [Som09, Roe02]. In order to fit the measurement points we make use of the three-term Sellmeier dispersion formula [Tat84]:

$$
n(\lambda)=\sqrt{1+\frac{B_{1} \lambda^{2}}{\lambda^{2}-C_{1}}+\frac{B_{2} \lambda^{2}}{\lambda^{2}-C_{2}}+\frac{B_{3} \lambda^{2}}{\lambda^{2}-C_{3}}}
$$

We chose to use three terms in the Sellmeier formula to have a better fit with the experimental results, obtaining a maximum error on the refractive index of $0.37 \times 10^{-}$ 3. When using only two terms the fit error was much larger $2.64 \times 10^{-3}$. The coefficients of (2.1) for which the best fit is obtained with the measurement results are given in Table 2.5, while in Fig 2.5 the measured refractive index values for both $\mathrm{TE}$ and TM polarizations, together with the respective fitting functions, are shown as a function of wavelength. 
Table 2.5 - Coefficients of the Sellmeier dispersion formula for $\mathrm{SiO}_{2}$

\begin{tabular}{cll}
\hline & \multicolumn{1}{c}{ TE } & \multicolumn{1}{c}{ TM } \\
\hline $\boldsymbol{B}_{\mathbf{1}}$ & $702.57 \times 10^{-3}$ & $758.36 \times 10^{-3}$ \\
$\boldsymbol{B}_{\mathbf{2}}$ & $408.60 \times 10^{-3}$ & $354.93 \times 10^{-3}$ \\
$\boldsymbol{B}_{\mathbf{3}}$ & $1164.35 \times 10^{-3}$ & $839.55 \times 10^{-3}$ \\
$\boldsymbol{C}_{\mathbf{1}}\left(\boldsymbol{\mu m}^{2}\right)$ & $3.67 \times 10^{-3}$ & $7.07 \times 10^{-3}$ \\
$\boldsymbol{C}_{\mathbf{2}}\left(\boldsymbol{\mu m}^{2}\right)$ & $11.72 \times 10^{-3}$ & $6.81 \times 10^{-3}$ \\
$\boldsymbol{C}_{\mathbf{3}}\left(\boldsymbol{\mu m}^{2}\right)$ & 110.21 & 81.97 \\
\hline
\end{tabular}

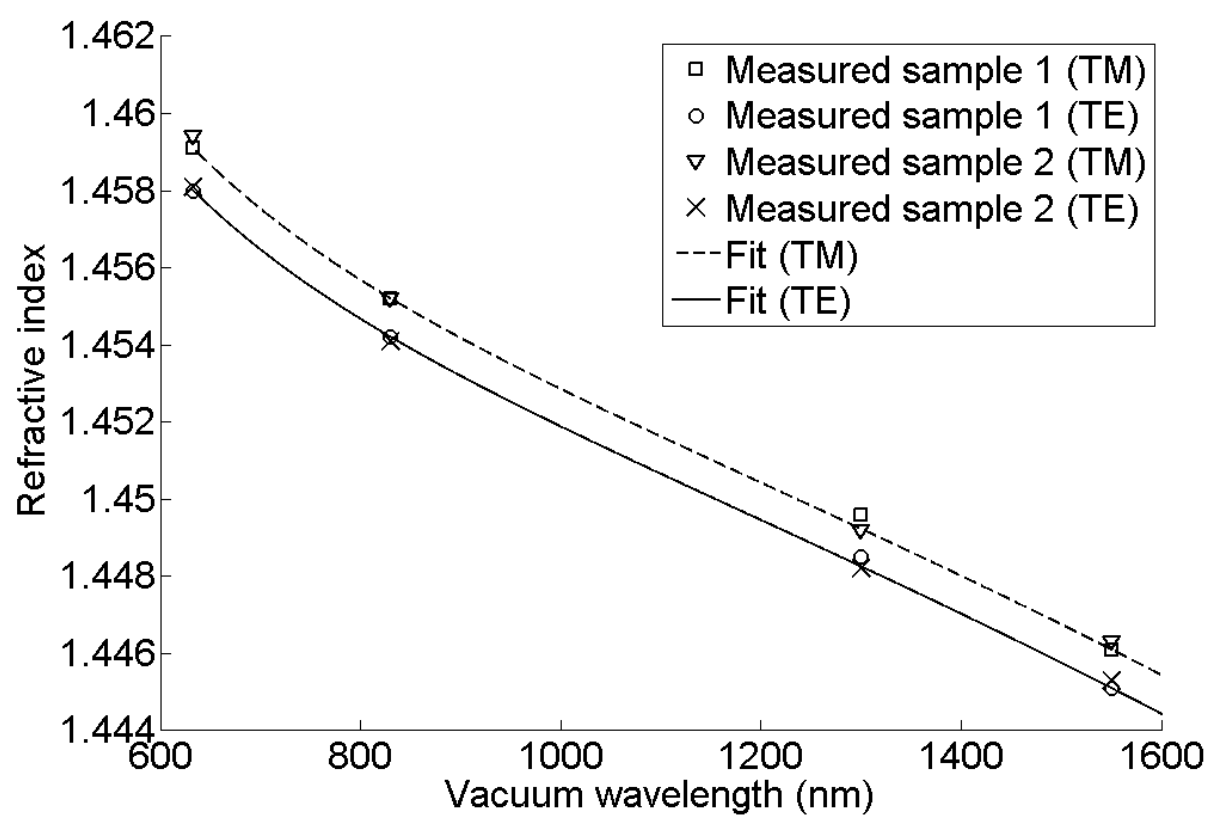

Fig. 2.5 - Refractive index measurements on two different oxidized silicon wafers, and calculated Sellmeier fitting functions for TE and TM polarizations.

In Fig. 2.5 we can clearly observe that the slope of the refractive index curve increases at higher wavelengths $(>1.3 \mu \mathrm{m})$ as expected for silica which presents a minimum of the material dispersion near $1.3 \mu \mathrm{m}$ [Mal65].

In order to model SiON we had to take into account that its refractive index (at a specified wavelength) varies depending on the $\mathrm{O} / \mathrm{N}$ ratio in the deposited layer. For this reason we aimed for a model that could predict the dispersion curve of the material (refractive index values vs. wavelength) based on the value of the refractive index at one specific wavelength (reference wavelength). We used $830 \mathrm{~nm}$ as our reference wavelength. 
To implement the model we relied on refractive index measurements performed on three different layers. The layers were grown on $\mathrm{SiO}_{2}$ using different $\mathrm{N}_{2} \mathrm{O} / \mathrm{SiH}_{4}$ flow ratios to achieve different values of refractive index in the range $1.518-1.526$ at $830 \mathrm{~nm}$. The deposition was performed using the Oxford 80 Plasmalab machine. All three layers were deposited using the same settings for the frequency and power of the RF supply which were $187.5 \mathrm{kHz}$ and $60 \mathrm{~W}$, respectively, as well as the same chamber pressure $\mathrm{P}=650 \mathrm{mTorr}$, process temperature $\mathrm{T}=300^{\circ} \mathrm{C}$, and $\mathrm{SiH}_{4} / \mathrm{N}_{2}$ flow of $600 \mathrm{sccm}$. The only parameters that were changed in the deposition of the three layers were the $\mathrm{N}_{2} \mathrm{O}$ flow (which determined the change in refractive index) and the deposition time (which determined the layer thickness). In particular, the $\mathrm{N}_{2} \mathrm{O}$ flow was varied between $280 \mathrm{sccm}$ and $370 \mathrm{sccm}$.

Each refractive index measurement (see Fig. 2.6) was performed for both polarizations at the four different wavelengths $(632.8,830,1300$ and $1550 \mathrm{~nm})-$ these are the wavelengths available in the Metricon setup.

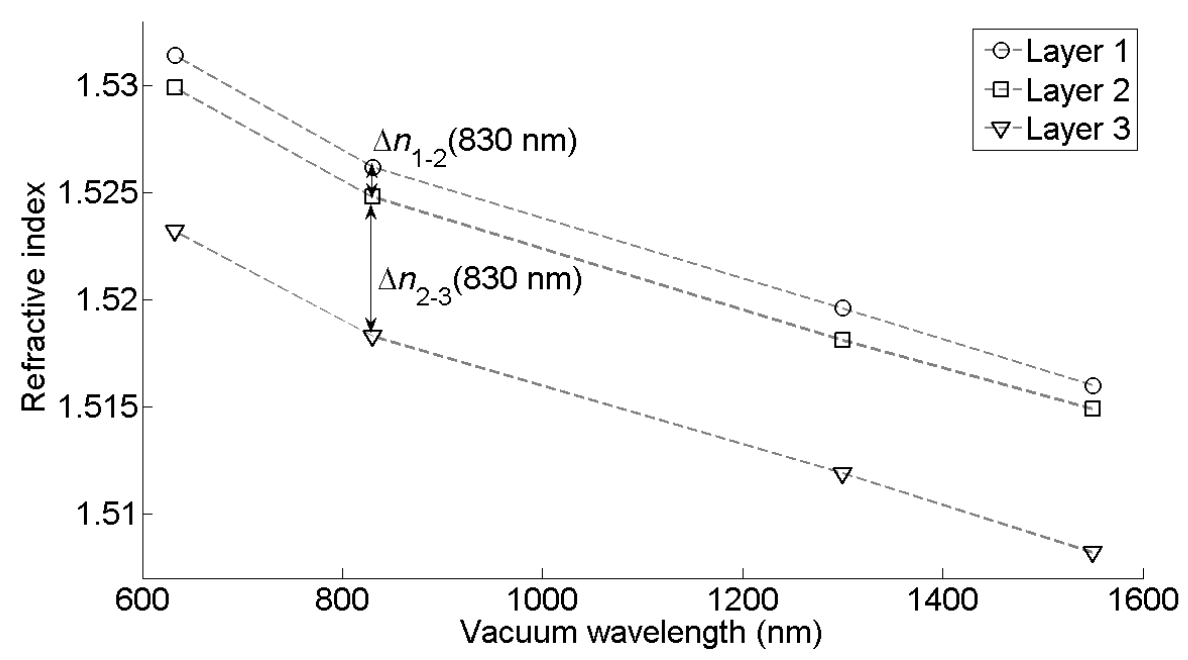

Fig. 2.6 - Refractive index measurements on three different SiON layers for TE polarization and for four different wavelengths $(632.8,830,1300$ and $1550 \mathrm{~nm})$.

From the measurements in Fig. 2.6, we observed that the three different dispersion curves are almost parallel to each other. We could also verify that by fitting the refractive index measurements on one of the layers with the Sellmeier dispersion formula of the form (2.1), the measurements on the other layers could be fitted by simply adding a constant offset to the calculated curve, leading to an acceptable fitting error (smaller than the measurement error). In particular, by fitting the measurement points acquired on layer 2 we obtained a reasonable fit (maximum error of $3.1 \times 10^{-4}$ ) for the points of layer 1 by adding the difference between the refractive index values at $830 \mathrm{~nm}\left|\Delta n_{1-2}\right|$ (see Fig. 2.6), and similarly for the points of layer 3 (maximum error of $3.8 \times 10^{-4}$ ) by subtracting $\left|\Delta n_{2-3}\right|$. The fitting errors 
obtained are smaller than the measurement errors obtained using the Metricon prism coupling setup $\left(\sim 1 \times 10^{-3}\right)$. The Sellmeier coefficients for layer 2 are given in Table 2.6 for TE polarization.

\begin{tabular}{cc}
$\begin{array}{c}\text { Table 2.6 - Coefficients of the Sellmeier dispersion } \\
\text { formula for SiON (layer 2) for TE polarization }\end{array}$ \\
\hline $\boldsymbol{B}_{\mathbf{1}}$ & $844.71 \times 10^{-3}$ \\
$\boldsymbol{B}_{\mathbf{2}}$ & $452.19 \times 10^{-3}$ \\
$\boldsymbol{B}_{\mathbf{3}}$ & $834.56 \times 10^{-3}$ \\
$\boldsymbol{C}_{\mathbf{1}}\left(\boldsymbol{\mu \mathbf { m } ^ { 2 } )}\right.$ & $4.59 \times 10^{-3}$ \\
$\boldsymbol{C}_{\mathbf{2}}\left(\boldsymbol{\mu \mathbf { m } ^ { 2 } )}\right.$ & $15.15 \times 10^{-3}$ \\
$\boldsymbol{C}_{\mathbf{3}}\left(\boldsymbol{\mu ~ m}^{2}\right)$ & 77.46 \\
\hline
\end{tabular}

From the measurements performed on the three layers we verified that the birefringence of SiON is $\sim 1 \times 10^{-3}$ and does not change after annealing. In Fig. 2.7 we show the fits and measured data points for both polarizations for layers 2 and 3.

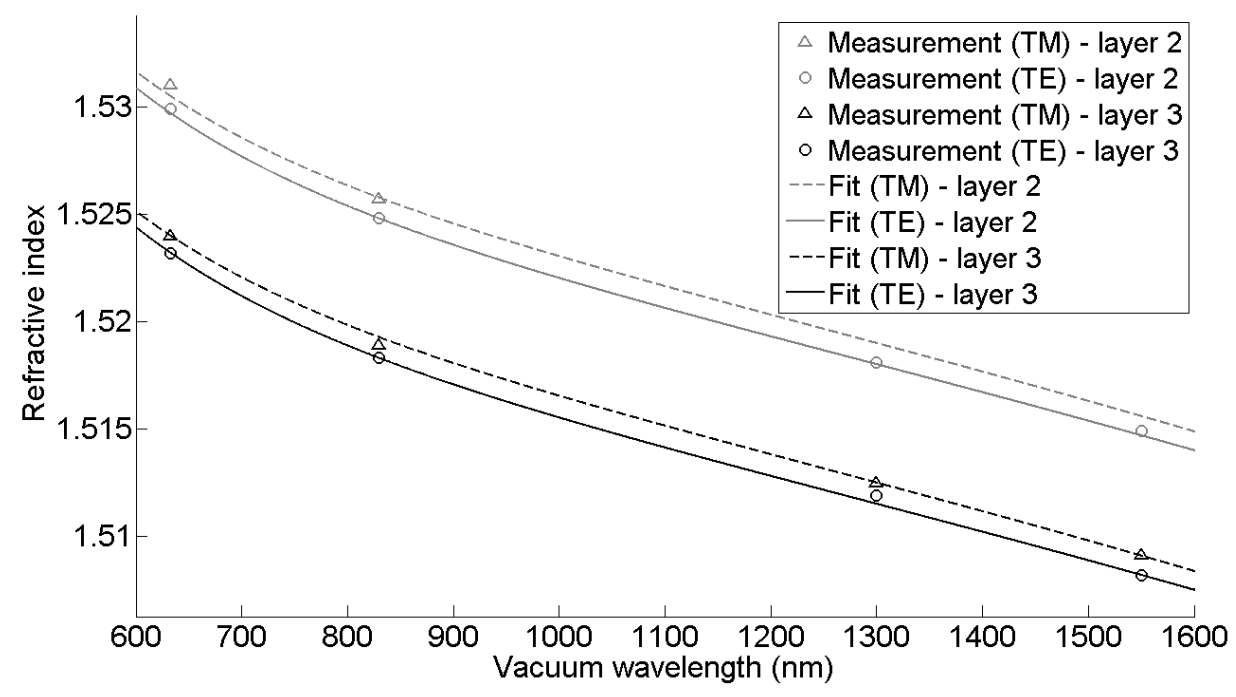

Fig. 2.7 - Refractive index measurements on two different SiON layers, and calculated Sellmeier fitting functions for TE and TM polarizations.

\subsection{Waveguide design}

The channel waveguide is the basic building block used in all the integrated filters described in this thesis. For the filters to work properly the waveguides must be single-mode over the entire operating wavelength region. For this reason we designed different waveguides for the different applications targeted in our project. An important design parameter of the waveguides is the channel birefringence, which is defined as the difference between the effective index for the TM and TE 
polarizations. For the skin applications, the channel birefringence must be small (preferably zero) in order for the wavelength selection filters to be independent of the polarization [Wör07]. On the other hand, if the wavelength selection filters worked for only one of the two polarizations, then we would need to filter out the other one, thereby losing half of the (already weak) Raman signal. For the dental application the requirements are different, since here we need to separate the TE and TM polarizations and analyze them using two distinct wavelength selection filters. Therefore, a low channel birefringence is not required in this case.

\subsubsection{Waveguides for the skin applications (detection of water and NMF concentrations)}

The two skin applications, as described in Chapter 1, have similar specifications in terms of the Raman signal to be detected. In both cases the Raman signal is located between $800 \mathrm{~nm}$ and $920 \mathrm{~nm}$. The main difference between the two applications is that for the detection of water concentration (application 1) an excitation wavelength of $671 \mathrm{~nm}$ is needed, while for the detection of the NMF concentration (application 2) the excitation wavelength is $785 \mathrm{~nm}$. In order for the laser suppression filters to work properly the waveguides must be single-mode not only in the spectral region between $800 \mathrm{~nm}$ and $920 \mathrm{~nm}$, but also at the excitation wavelengths. In the case of application 1 the requirement that the waveguide is single-mode in the wavelength region between $671 \mathrm{~nm}$ and $920 \mathrm{~nm}$, while still maintaining low bending losses at $920 \mathrm{~nm}$ and having low birefringence between $800 \mathrm{~nm}$ and $920 \mathrm{~nm}$ is not practical, since it leads to an excessively small waveguide width and large bending radii. For this reason we relaxed the requirements, aiming at a waveguide structure that is single-mode in the wavelength region from $785 \mathrm{~nm}$ to $920 \mathrm{~nm}$, which is compatible with both applications, provided that an external laser suppression filter is used in application 1. The channel waveguide shown in Fig. 2.8 was designed taking into account the fabrication tolerances (on the waveguide width, layer thickness and refractive index) with the following specifications:

\section{Design:}

- Dimensions

waveguide width: $w=2 \mu \mathrm{m} \pm 0.1 \mu \mathrm{m}$, waveguide height: $h=0.52 \mu \mathrm{m} \pm 0.03 \mu \mathrm{m}$.

- Refractive index for TE polarization core: $n_{\text {core }} \cong 1.509 \pm 1 \times 10^{-3}$ at $830 \mathrm{~nm}$, cladding: $n_{\text {clad }} \cong 1.454 \pm 1 \times 10^{-3}$ at $830 \mathrm{~nm}$.

The properties of this design are:

- Single-mode operation in the wavelength region from $785 \mathrm{~nm}$ to $920 \mathrm{~nm}$.

- Channel birefringence between $-0.5 \times 10^{-4}$ and $1.5 \times 10^{-4}$.

- Bending losses of $0.1 \mathrm{~dB} / \mathrm{cm}$ for a bending radius of $1300 \mu \mathrm{m}$. 


\section{TEOS + PECVD oxide}

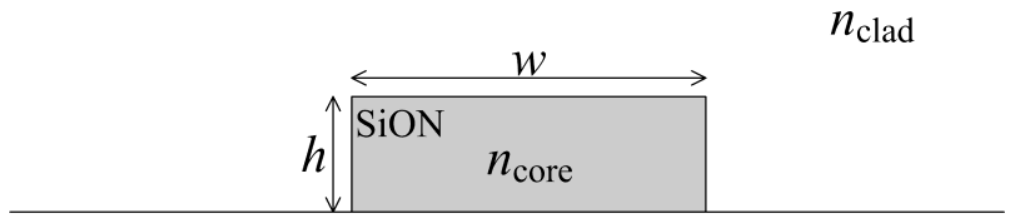

Thermal oxide

$$
n_{\text {clad }}
$$

Fig. 2.8 - Channel waveguide cross-section.

The calculated channel birefringence is shown in Fig. 2.9 as a function of wavelength.

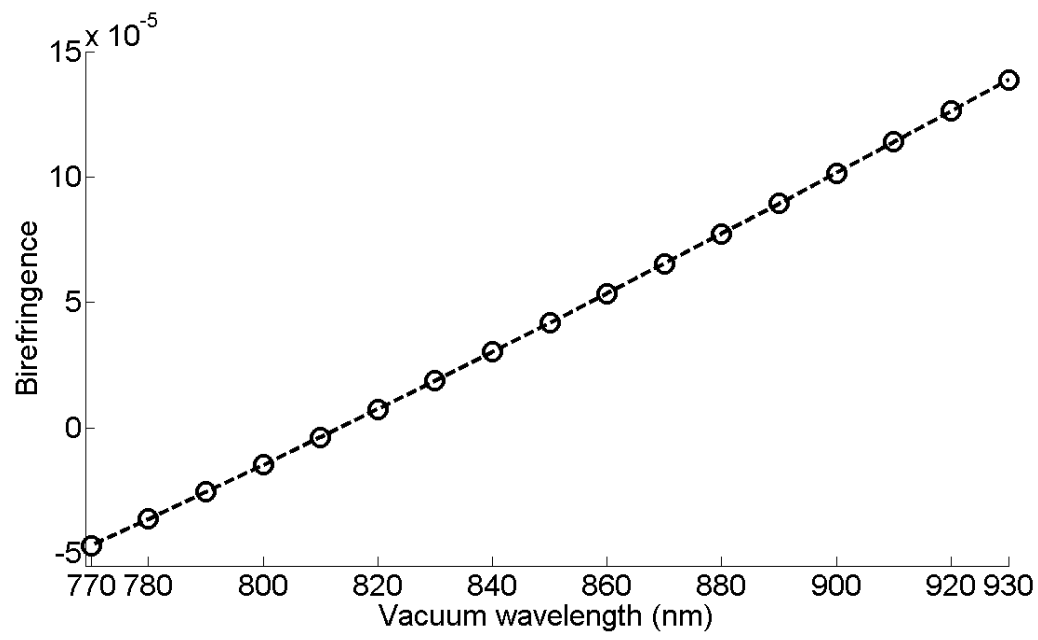

Fig. 2.9 - Calculated channel birefringence as a function of wavelength.

The bending losses for the designed channel waveguides are shown in Fig. 2.10 as a function of the bending radius. The bending losses are calculated at the highest wavelength in the spectral region of interest by taking into account the fabrication tolerances. The simulations were performed using the Phoenix Field Designer 2D bend mode solver [http://www.phoenixbv.com/]. From the simulations we observe that for bending radii as small as $1300 \mu \mathrm{m}$ the bending losses are $\sim 0.1 \mathrm{~dB} / \mathrm{cm}$, corresponding to $0.02 \mathrm{~dB} / 90^{\circ}$. By including a safety margin, we chose a minimum bending radius of $1700 \mu \mathrm{m}$, allowing us to assume the pure bending losses in the fabricated waveguide bends to be lower than $0.1 \mathrm{~dB} / \mathrm{cm}$. 


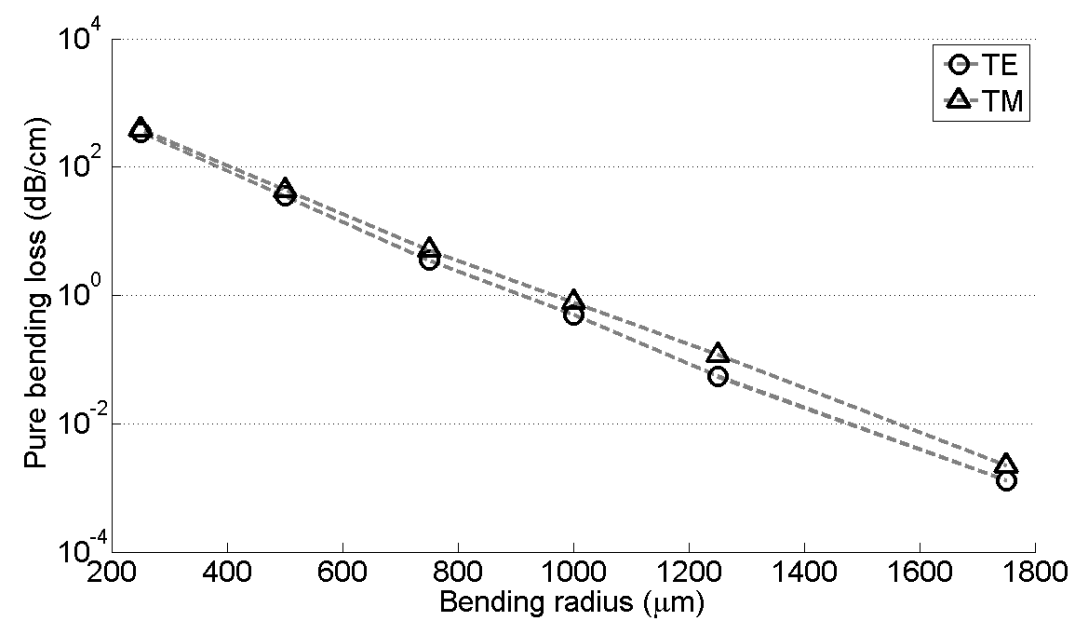

Fig. 2.10 - Calculated pure bending losses as a function of bending radius at $930 \mathrm{~nm}$.

In order to obtain a high coupling efficiency between the on-chip devices and the fibers used in the measurement setup we designed on-chip spot size converters in which we used lateral tapering [Rid98, Roe02] to adapt the mode diameter in the waveguides to that of a small core fiber with a mode field diameter (MFD) of 5.3 $\mu \mathrm{m}$. The simulations were performed by calculating the overlap integrals between the modes in the fiber and the channel waveguide for different values of the waveguide width. In particular, in Fig. 2.11 the coupling efficiency is shown as a function of the taper width for both TE and TM polarizations at a wavelength of 881 $\mathrm{nm}$, which corresponds to the central wavelength of one of the Raman bands to be detected in the NMF application (see Table 1.1). In this case we calculated a maximum fiber-to-chip coupling efficiency of $\sim 74 \%$ for a taper width of $\sim 0.8 \mu \mathrm{m}$.

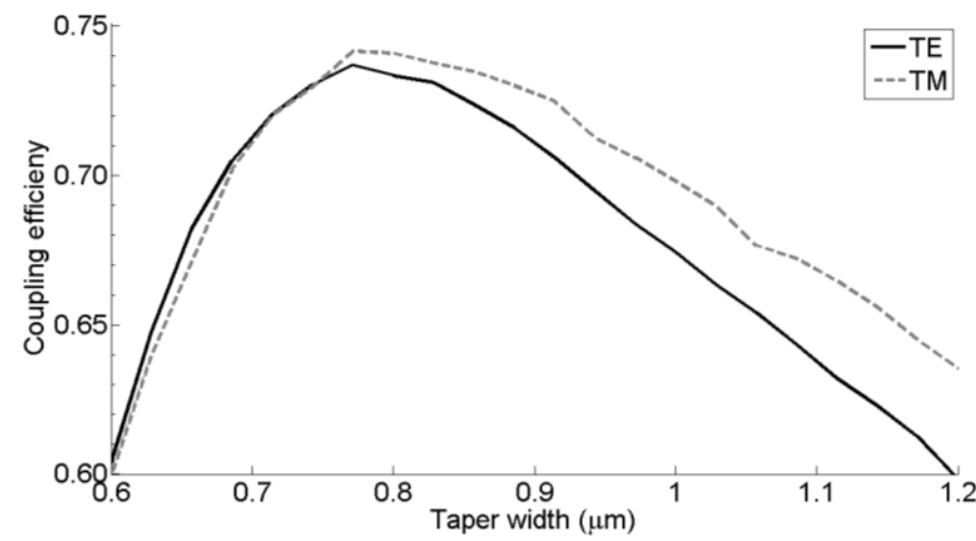

Fig. 2.11 - Calculated fiber-to-chip coupling efficiency as a function of the waveguide width $w$, for both transverse-electric (TE) and transverse-magnetic (TM) polarizations at $881 \mathrm{~nm}$. 
In Fig. 2.12 we show the coupling efficiency (at the chosen width of $0.8 \mu \mathrm{m}$ ) as a function of fiber displacement in both $x$ and $y$ directions and for both polarizations. We observe that the fiber must be aligned to the chip with a maximum positioning error of $\pm 1.7 \mu \mathrm{m}$ in both the $x$ and $y$ directions in order for the coupling efficiency to be within $50 \%$ of the maximum value. The required alignment precision is easily achievable with most of the alignment stages found in our lab, which have submicrometer precision.
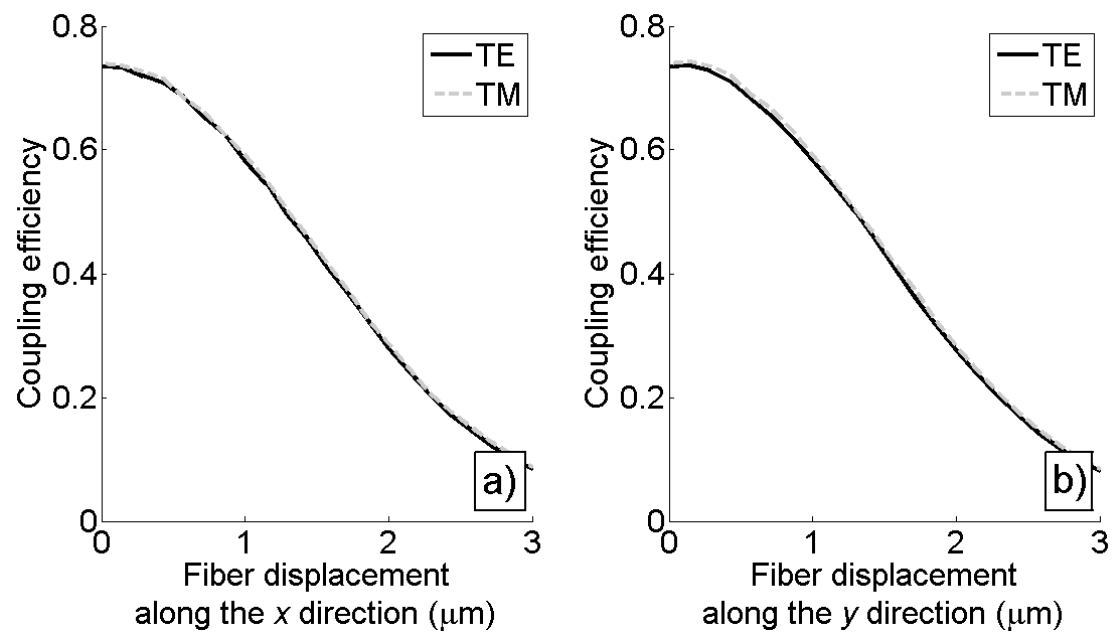

Fig. 2.12 - Fiber-to-chip coupling efficiency at $881 \mathrm{~nm}$ as a function of the fiber displacement.

\subsubsection{Waveguides for the dental application (detection of early dental caries)}

In the detection of early dental caries the wavelength of the excitation laser is 830 $\mathrm{nm}$, while the Raman signal is located in the wavelength region between $890 \mathrm{~nm}$ and $912 \mathrm{~nm}$. Consequently, for the wavelength selection and laser suppression filters to work properly the single mode condition for the channel waveguides must be met in the entire spectral region between $830 \mathrm{~nm}$ and $912 \mathrm{~nm}$. The designed channel waveguide for the dental application has the following specifications:

\section{Design:}

- Dimensions

waveguide width: $w=2.25 \mu \mathrm{m} \pm 0.1 \mu \mathrm{m}$,

waveguide height: $h=0.52 \mu \mathrm{m} \pm 0.03 \mu \mathrm{m}$.

- Refractive index for TE polarization

core: $n_{\text {core }} \cong 1.509 \pm 1 \times 10^{-3}$ at $830 \mathrm{~nm}$,

cladding: $n_{\text {clad }} \cong 1.454 \pm 1 \times 10^{-3}$ at $830 \mathrm{~nm}$. 
The properties of this design are:

- Single-mode operation in the wavelength region from $830 \mathrm{~nm}$ to $912 \mathrm{~nm}$.

- Channel birefringence between $3.5 \times 10^{-5}$ and $7.8 \times 10^{-5}$.

- Bending losses of $0.1 \mathrm{~dB} / \mathrm{cm}$ for a bending radius of $1000 \mu \mathrm{m}$.

Also in this case to optimize the fiber-to-chip coupling, an on-chip spot-size converter was designed and optimized for a wavelength of $901 \mathrm{~nm}$. The optimized taper presented the same width as the one for the dental application, and also the same performance in terms of maximum coupling efficiency (74\%). This time the alignment accuracy requirement was $\pm 1.8 \mu \mathrm{m}$.

In Table 2.7 we show the results on refractive index and thickness measurements performed using the Metricon setup on $\mathrm{SiON}$ layers deposited on $\mathrm{SiO}_{2}$. The measurements were carried out on 11 different wafers. In the table we indicate with $n_{\text {eff }}$ the measured effective index (approximated to the fourth digit), with $n_{\mathrm{d}}=520 \mathrm{~nm}$ the refractive index of the layer estimated imposing a layer thickness of $520 \mathrm{~nm}$, and with $d_{\mathrm{n}=1.509}$ the layer thickness estimated imposing a refractive index value for the $\mathrm{SiON}$ layer of 1.509. In fact, since the SiON layer is single mode it is impossible to measure both the refractive index and the thickness using Metricon.

Table 2.7 - Effective index measurements on deposited SiON layers for the skin and dental applications

\begin{tabular}{|c|c|c|c|}
\hline Wafer \# & $\boldsymbol{n}_{\text {eff }} @ \mathbf{8 3 0} \mathbf{~ n m}$ & $\boldsymbol{n}_{\mathbf{d}=\mathbf{5 2 0} \mathbf{~ n m}}$ & $\boldsymbol{d}_{\mathbf{n}=1.509}(\mathbf{n m})$ \\
\hline $\mathbf{2 3 2 1 9 2 2 1 9 6}$ & 1.4593 & 1.5102 & 528.6 \\
\hline $\mathbf{2 3 2 1 9 2 2 2 1 2}$ & 1.4600 & 1.5117 & 540.3 \\
\hline $\mathbf{2 3 2 1 9 2 2 2 1 7}$ & 1.4600 & 1.5117 & 540.3 \\
\hline $\mathbf{2 3 2 1 9 2 2 2 2 0}$ & 1.4597 & 1.5109 & 534.5 \\
\hline $\mathbf{2 3 6 8 5 7 5 0 8 4}$ & 1.4597 & 1.5111 & 535.6 \\
\hline $\mathbf{2 3 6 8 5 7 5 0 9 2}$ & 1.4605 & 1.5128 & 548.6 \\
\hline $\mathbf{2 3 6 8 5 7 5 0 9 7}$ & 1.4594 & 1.5103 & 529.7 \\
\hline $\mathbf{2 3 6 8 5 7 5 0 9 8}$ & 1.4595 & 1.5107 & 532.7 \\
\hline $\mathbf{2 3 6 8 5 7 5 1 0 0}$ & 1.4596 & 1.5109 & 534.1 \\
\hline $\mathbf{2 3 6 8 5 7 5 1 5 9}$ & 1.4593 & 1.5101 & 528.2 \\
\hline $\mathbf{2 3 6 8 5 7 5 1 7 1}$ & 1.4595 & 1.5107 & 532.7 \\
\hline
\end{tabular}

\subsubsection{The effective index method}

The effective index method (EIM) is a well known technique for approximating a 2D transversal index distribution with an equivalent 1D distribution. It enables one to reduce the computational effort and time required in mode calculations and beam propagation methods. There are many variations of the EIM presented in the literature [Hoc77, Mun91, Wan06]. A simple form of the EIM, which can be applied to a rib waveguide structure, as the one shown in Fig. 2.13 (a), is described in [Kaw01], and is based on the slab waveguide analysis. 

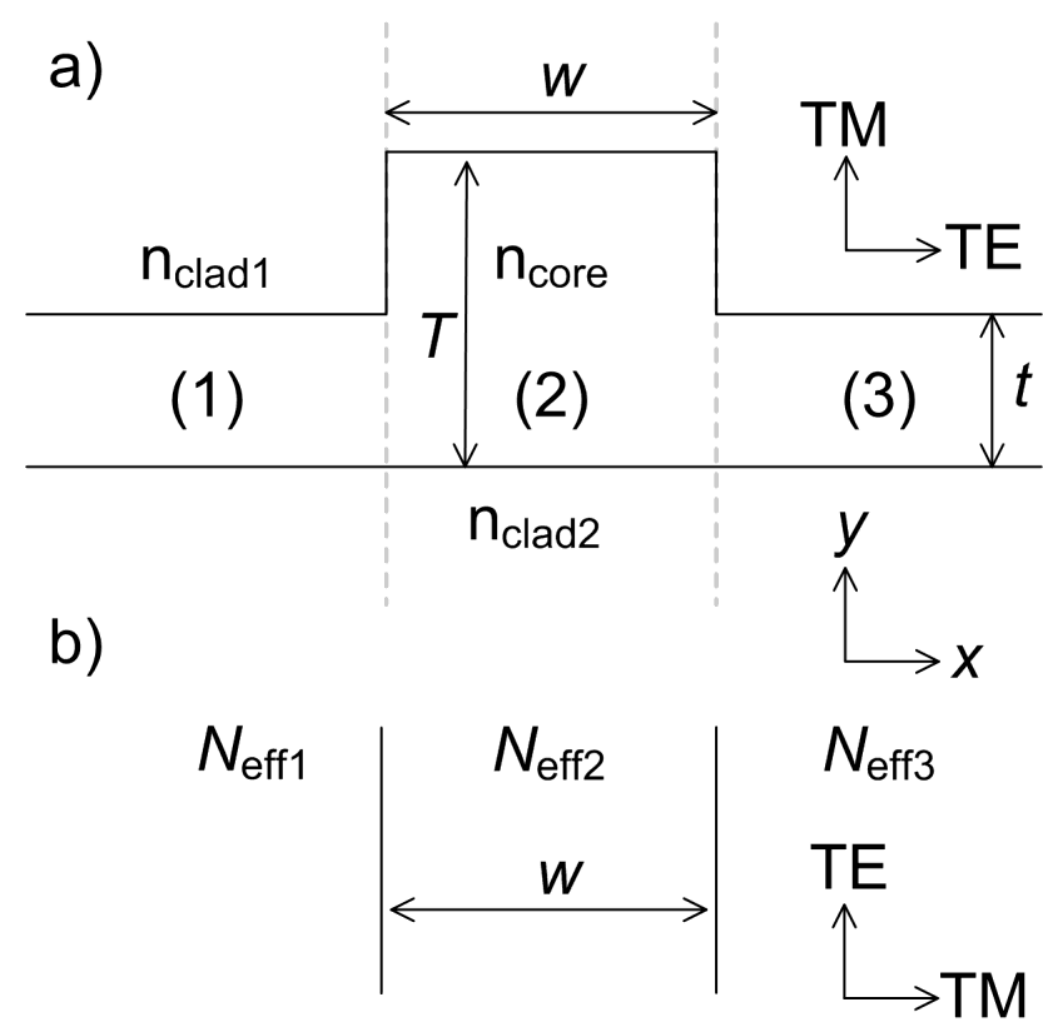

Fig. 2.13 - a) Generic rib waveguide structure with width $w$, height $T$, and etch depth $T$ - $t$; b) the equivalent slab waveguide obtained with the effective index method. The arrows labeled as TE and TM indicate the direction of the transverse-electric and transversemagnetic polarizations as conventionally assumed in rib and slab waveguides.

In order to calculate the effective index of the rib waveguide for the TE polarization, four steps are generally performed:

1. The waveguide is separated into three parts as represented in Fig. 2.13 (a).

2. Each part is considered as a $1 \mathrm{D}$ optical waveguide and its effective index is calculated for the TE polarization.

3. An equivalent slab waveguide is considered, formed by the three previously calculated effective indices (see Fig. 2.13 (b)).

4. Finally, the effective index of the equivalent slab waveguide is computed for the TM polarization (since by convention for a slab waveguide an electric field with TE polarization is oriented parallel to the layer stack).

This method only works if the regions (1) and (3) outside the rib (see Fig. 2.13 (a)) support guided modes. Therefore, in case of an embedded channel waveguide, such as designed in 2.4.1 and 2.4.2, this method will not work. 
In order to apply the EIM to our channel waveguides we adopted a numerical approach based on the methods proposed by Munowitz and Wang [Mun91, Wan06]. In the first method the refractive indices of core and cladding of the equivalent planar waveguide, as well as its width (this parameter can also be fixed) are optimized (numerically) by imposing the following three conditions at the desired wavelength (and polarization):

1. That the number of guided modes in the equivalent planar waveguide is equal to the number of guided modes in the original channel waveguide.

2. That the effective indices of the guided modes in the planar waveguide match those of the channel waveguides.

3. That the difference between the electric field in the planar waveguide $E_{\text {planar }}(x)$ and the projection along the $x$ direction of the electric field in the channel waveguide $E_{\text {proj }}(x)$ is zero, where both fields are first normalized to unit integrated intensity.

The second method [Wan06] is an EIM for directional couplers. This method enables to replace a directional coupler with an equivalent coupler based on slab waveguides. The numerical procedure involves the optimization of the cladding refractive index in the equivalent planar coupler in order to achieve the same coupling length as that of the original coupler. In this case the refractive index of the core of the equivalent slab structure is calculated using the standard EIM method as described in [Kaw01].

We performed simulations on the coupler geometries shown in Fig. 2.14.

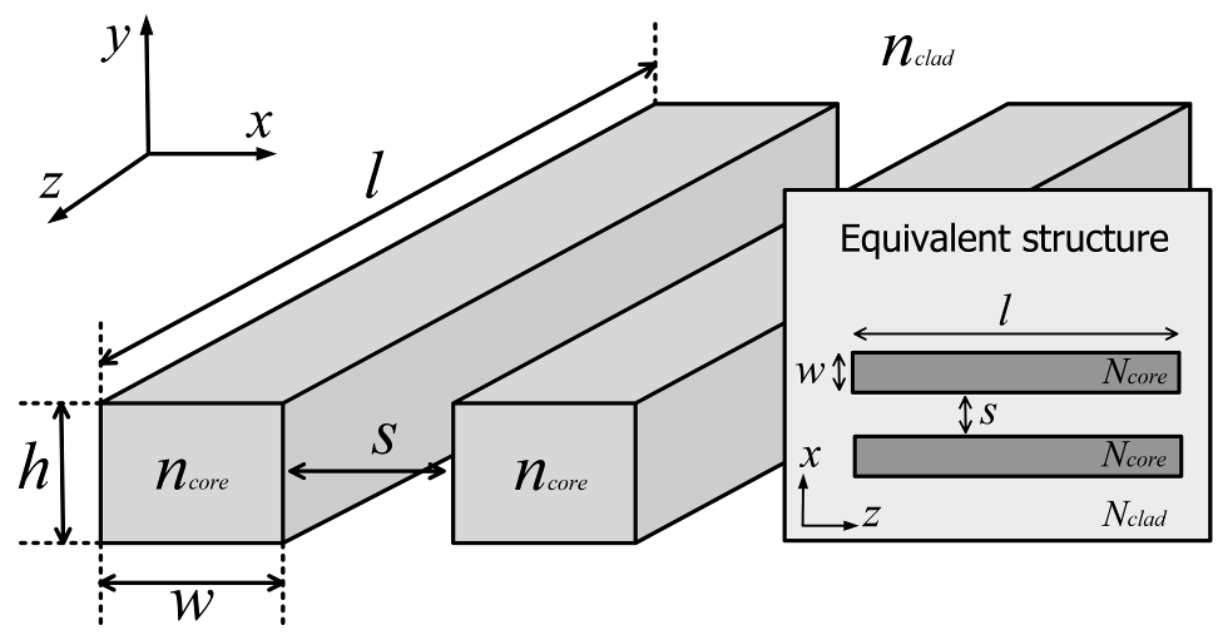

Fig. 2.14 - Directional coupler geometry used for 3D beam-propagation-method (BPM). In the inset the equivalent structure used in 2D BPM is shown (the structure extends infinitely in the $y$ direction). We indicate with $w$ and $h$ the width and height of the coupler waveguides; with $s$ the separation between the waveguides, and with $l$ the length of the coupler. 
We found that the previous two methods have limited applicability. For example, using the first method to simulate directional couplers we obtained a large discrepancy between the coupling lengths calculated with $2 \mathrm{D}$ and 3D beam propagation methods (BPM). First we used the EIM to calculate the equivalent slab waveguide for the channel waveguide designed for the skin application. After optimization the following values for the index of core and cladding of the equivalent waveguide were found: $N_{\text {core }}=1.47236, N_{\text {clad }}=1.45314$, while for the width we opted to use a fixed value of $2 \mu \mathrm{m}$. With these values the difference between the effective indices of the channel waveguide and slab waveguide was only $\Delta N_{\text {eff }}=1.46666-1.46661=5 \times 10^{-5}$. Then we simulated with both $3 \mathrm{D}$ BPM and 2D BPM four directional couplers having different values of the separation $s$ between the waveguides $(s=1 \mu \mathrm{m}, s=1.5 \mu \mathrm{m}, s=2 \mu \mathrm{m}$, and $s=2.5 \mu \mathrm{m})$. For each coupler we plotted the normalized intensity in one of the output branches as a function of the coupler length. The results are shown in Fig. 2.15 for a wavelength of $850 \mathrm{~nm}$ and for TE polarization. We observe that the error, which is calculated as the difference in intensity between the 3D BPM and 2D BPM computations, oscillates significantly between -1 and 1 . The amplitude of these oscillations reduces as the separation $s$ between the waveguides increases. However, it is clear from our results that this EIM method cannot be applied to reliably simulate directional couplers.

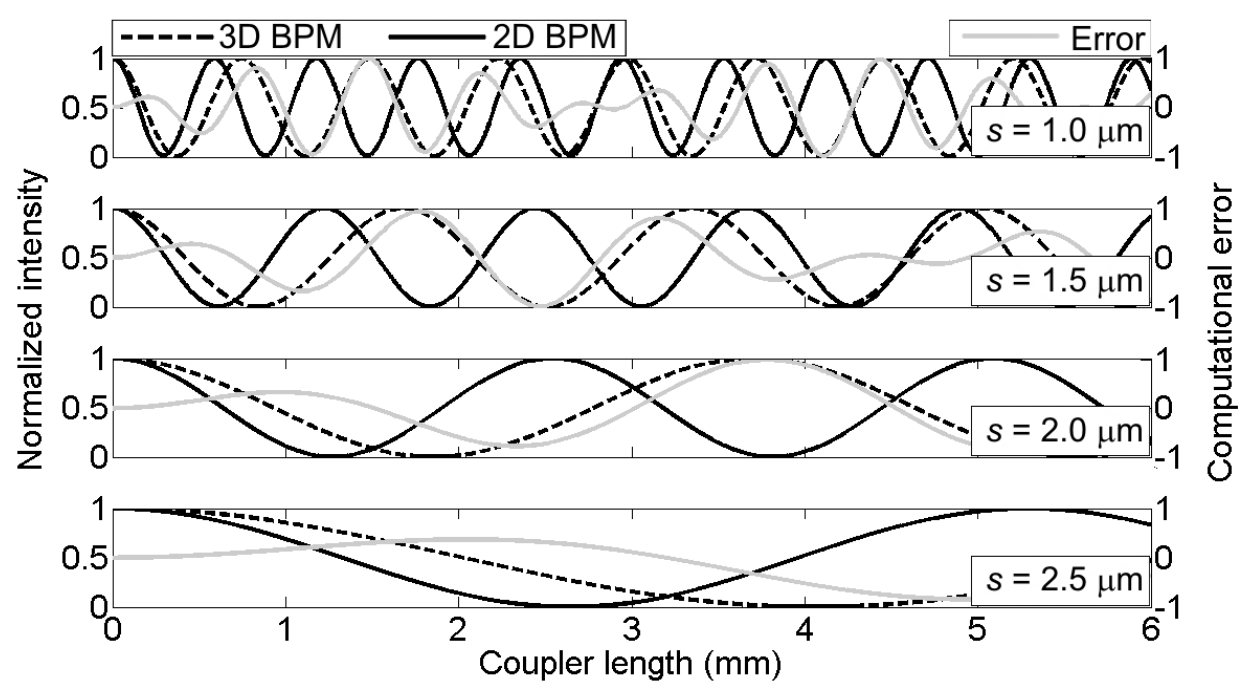

Fig. 2.15 - The normalized intensity in one of the output branches of a directional coupler as a function of the coupler length for four different values of the waveguide separation $s$. The dashed curve is the intensity computed using the 3D beam-propagation-method (BPM), while the solid black curve is the intensity computed using the 2D BPM. The difference between the intensities $(3 D-2 D)$ is represented by the gray line plotted against the right-hand axis. 
On the other hand, the second method [Wan06] is not applicable when the correct value of the effective index is needed. This is because by changing only the cladding index, while keeping the core index constant there is no control over the effective index of the guided mode.

In order to have more accurate results when simulating couplers, still maintaining the correct value for the propagation constant we developed a different method which can be considered as a combination of the previous two. In our method we perform a numerical optimization on the values of the core and cladding indices, as well as on the thickness of the slab waveguide by imposing the following conditions: Firstly, in order to preserve the correct propagation constant we impose that the equivalent slab waveguide has the same effective index as the channel waveguide (where both waveguides are single-mode). The second condition is imposed on the coupling length of two directional couplers, one having a center-tocenter separation between the waveguides $s=1 \mu \mathrm{m}$, and the other having $s=2 \mu \mathrm{m}$. In the optimization procedure we impose that the coupling lengths of these directional couplers calculated using 2D BPM on the equivalent structures are the same as those calculated using 3D BPM (372 $\mu \mathrm{m}$ for the first coupler, and $1854 \mu \mathrm{m}$ for the second one). After optimization the following values for the index of the core and cladding of the equivalent waveguide were found: $N_{\text {core }}=1.47300, N_{\text {clad }}=$ 1.44864; while the width of the equivalent slab waveguide was reduced by $0.047 \mu \mathrm{m}$ with respect to the original width of $2 \mu \mathrm{m}$. With these values the difference between the effective indices of the channel waveguide and equivalent slab waveguide was $\Delta N_{\text {eff }}=1.5 \times 10^{-4}$.

In Fig. 2.16 we show the results obtained by 3D BPM simulations on four different straight directional couplers at a wavelength of $850 \mathrm{~nm}$ and compared with the results of 2D BPM simulations (after optimization) of the equivalent slab structures. In the figure the intensity in one of the output branches of the directional coupler is plotted as a function of the coupler length. We observe that despite the fact that the couplers with $s=1.5 \mu \mathrm{m}$ and $s=2.5 \mu \mathrm{m}$ were not used in the optimization procedure the simulations conducted using 2D BPM on the equivalent structures are in good agreement with those conducted using 3D BPM. 


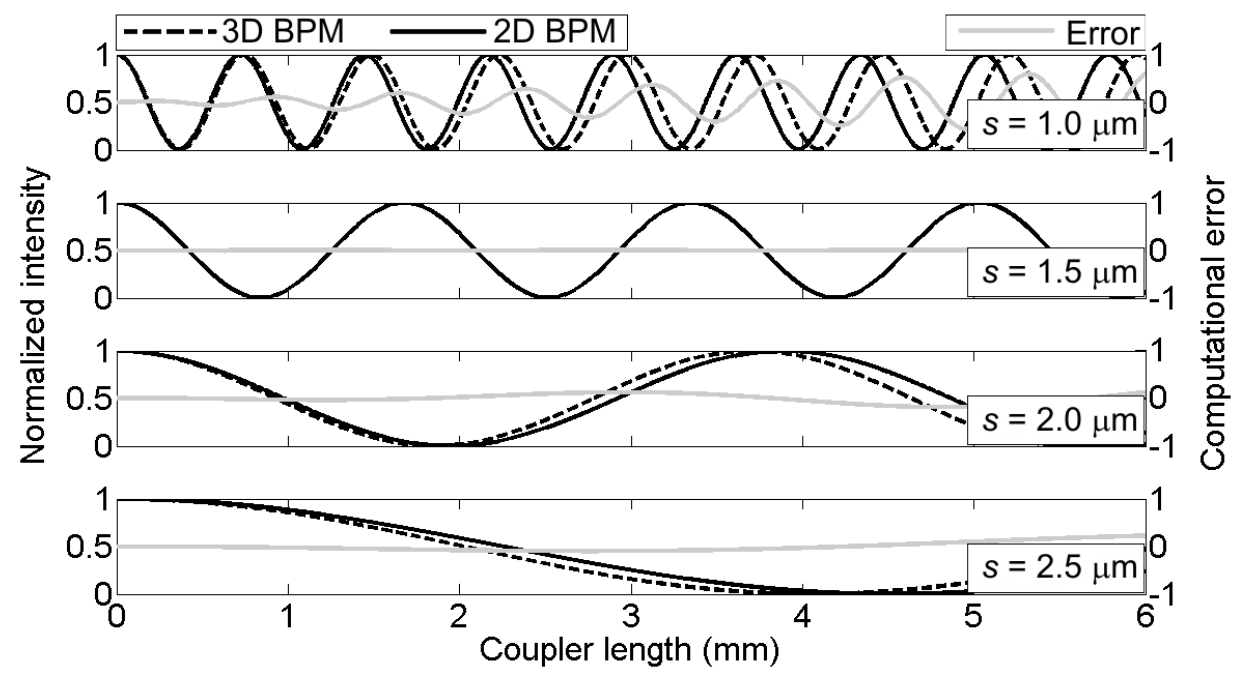

Fig. 2.16 - Normalized intensity in one of the output branches of a directional coupler as a function of the coupler length for four different values of the waveguide separation $s$. The dashed curve is the intensity computed using the 3D beam-propagation-method (BPM), while the solid black curve is the intensity computed using the 2D BPM. The difference between the intensities $(3 D-2 D)$ is represented by the gray line plotted against the right-hand axis.

In particular, we observe that for the coupler with waveguide separation $s=1.5$ $\mu \mathrm{m}$ which is in between the two selected values of $s=1 \mu \mathrm{m}$ and $s=2 \mu \mathrm{m}$ used for the optimization, the 3D and 2D BPM simulations give almost identical results. For the other values of waveguide separation the error is smallest for short coupler lengths (or equivalently for weak coupling). For this reason, in the design of the laser suppression filters, discussed in Chapter 5 and more in detail in the Appendix, we will use couplers with small power coupling ratios.

\subsection{Summary}

We described two fabrication processes of silicon oxynitride layers, and the corresponding optical properties of the fabricated layers. We discussed the modeling of the refractive indices of $\mathrm{SiON}$ and $\mathrm{SiO}_{2}$ using the Sellmeier dispersion formulas and presented the designs of channel waveguides used for the skin and dental applications. In the final Section of this Chapter we presented a modified effective index method which gives reliable results in simulating directional couplers and optical delay lines with 2D BPM. The fast and reliable simulation of these elements is necessary since they constitute the basic building blocks of the laser suppression filters described in Chapter 5. 


\section{Chapter 3}

\section{Signal collection with integrated waveguides}

\subsection{Introduction}

$\mathrm{T}$

he aim of our research, as already discussed in the introductory Chapter, is to realize a compact, portable, low-cost integrated device for the Raman

spectroscopy of biological samples. This implies that such a device must not only provide means to detect a Raman signal but also to deliver the excitation light to the sample under study. For this reason one of the key challenges of the Ramanpen project is the realization of an efficient interface between the sample under investigation and the on-chip optical devices.

To interface the device with the sample one could think of using fiber-optic probes. These represent an excellent solution for in-vivo and in-situ measurements, especially when small dimensions and high flexibility of the probe are required [Utz03]. A well-known problem with single-fiber probes is the luminescence and Raman emission induced in the fiber by reflected or Rayleigh-backscattered light from the laser excitation source, that may corrupt the weak Raman signals from the sample [Kon02]. Therefore, usually distinct fibers are used for excitation light delivery and signal light collection [Cas01, Lew96]. Fiber probes with different geometries have been studied and theoretical models have been introduced [Sch84, Pla86, Coo96] to compare different Raman probe designs. In our work we study the feasibility of using integrated waveguide probes instead of fiber probes. Among the advantages of integrated probes, besides the compactness, is that these can be placed on the same chip together with filters and other wavelength selective devices and by hybrid integration even with detectors and the necessary electronic circuits. Furthermore, integrated waveguide probes have the advantages of polarization maintenance and reduced propagation length, providing a significant reduction of the background noise. In addition, integrated probes can provide an order-ofmagnitude better resolution (smaller collection volumes) than fiber probes. As an example, the typical collection volume of a fiber probe is around $\sim 1.9 \times 10^{-4} \mathrm{~cm}^{3}$, as can be seen from columns 6 and 9 of TABLE I in Ref. [Coo96], whereas the integrated probes discussed in this thesis have a collection volume between $3.9 \times 10^{-9}$ and $2.6 \times 10^{-8} \mathrm{~cm}^{3}$, see Section 3.2.3.

In this Chapter we investigate the use of integrated optical waveguide probes as an alternative to fiber-optic probes. In particular, we compare their collection efficiency with those of large-core multi-mode and small-core single- and multimode fiber probes. For this purpose we make use of a semi-analytical model (described in the next Section) that we developed for integrated waveguide probes with an arbitrary cross-section, under the condition that the sample is a weakly 
scattering medium. In the next Section we will also discuss the probe design and show the simulation results obtained using our model. The model is validated by performing an experiment on the detection of fluorescence from a ruby rod through an integrated probe and comparing the results to those of the model.

The case of highly scattering media is discussed in Section 3.3, where we use Monte-Carlo simulations to predict the collection efficiency of the probe as a function of the separation between the collection and excitation waveguides. These simulations are validated by measurements of light backscattered from a water suspension of latex nanospheres, as shown in the final Section of this Chapter.

\subsection{Integrated waveguide probes for backscattered light collection from weakly scattering media}

In the following we consider weakly scattering media, for which the scattering coefficient $\mu\left[\mathrm{mm}^{-1}\right]$, given by the product of the scattering cross-section and the total number of scattering particles per unit volume, is such that $1 / \mu$ is on the order of the physical dimensions of the scattering domain. In this case photons that travel through the medium experience only one scattering event on average.

\subsubsection{Mathematical model of integrated waveguide probe}

In this work we consider probes with one excitation channel and one or more collection channels integrated within the same layer of a micro-chip. An example of such probes is shown in Fig. 3.1. The channels are equally spaced and all the collector waveguides are positioned on the same side with respect to the excitation waveguide. In this way it is possible to measure the backscattered light intensity as a function of lateral distance from the excitation point.

In order to model this kind of probes we consider the simple case of a dualwaveguide probe, as the one shown in Fig. 3.2, which is positioned at a distance $D$ from the surface of the sample under study. The probe is composed of one excitation waveguide and one collector waveguide, spaced a distance $d$ apart. Both integrated waveguides have necessarily the same height $h$, since they are etched from the same layer stack, but can be designed with different widths $w$. 


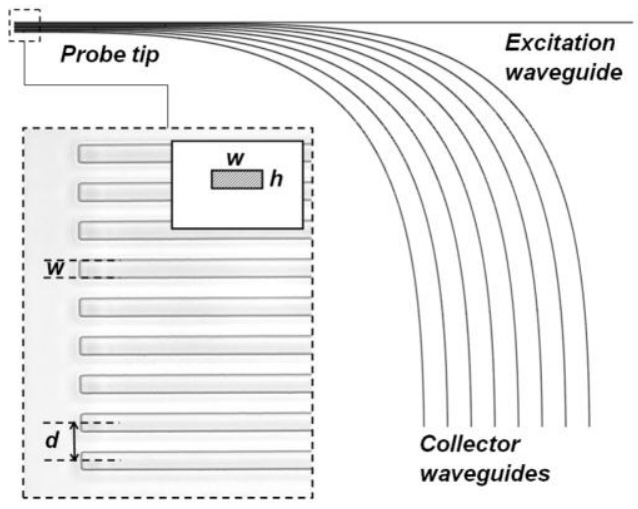

Fig 3.1 - Top view of a multi-waveguide integrated probe for backscattered light collection. An expanded view of the probe tip together with the waveguide cross-section is shown in the inset.

Generally, the light launched through the excitation waveguide into the sample will be diffracted differently in the horizontal and vertical directions, resulting in an elliptical cone of light spreading into the sample. Similarly, the collector waveguide will have an elliptical acceptance cone. The overlap region of these two cones determines the collection efficiency or "figure of merit" of the probe and its volume is highly dependent on the numerical apertures (NAs) of excitation and collector waveguides and also on the distance $D$ between probe and sample. The figure of merit for a fiber-optic probe with separate excitation and collection channels has been introduced in the work of Schwab et al. [Sch84]. In our work we have calculated this quantity for the geometry displayed in Fig. 3.2, in which the integrated waveguides have a rectangular cross-section and guide a number of modes that depends on their width, height and refractive index contrast.

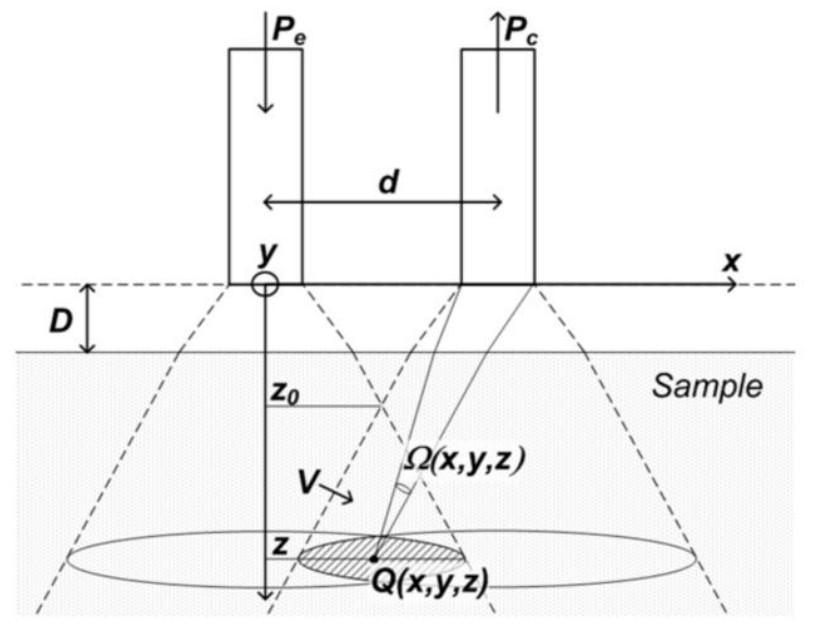

Fig 3.2 - Schematic of excitation and collector waveguides separated by the distance $d . P_{e}$ is the power coupled into the excitation waveguide and $P_{c}$ is the power coupled back into the collector waveguide. 
The irradiance $I(x, y, z)\left(\mathrm{W} / \mathrm{m}^{2}\right)$, calculated in a point $Q$ at coordinates $(x, y, z)$ that falls inside the excitation cone, is given by

$$
I(x, y, z)=P_{e} T_{e} \rho_{z}(x, y),
$$

where $P_{\mathrm{e}}$ is the laser power that is coupled into the excitation waveguide, $T_{\mathrm{e}}$ is the fraction of power transmitted into the sample from the excitation waveguide and $\rho_{z}(x, y)$ is the normalized power density distribution function $\left[\mathrm{m}^{-2}\right]$ of the waveguide's far field at distance $z$. In first-order approximation we consider that the excitation field spreads out as it propagates, forming an elliptical cone and that a fixed depth $z$ the modulus of the electric field follows a Gaussian distribution. In this case $\rho_{z}(x, y)$ can be represented by

$$
\rho_{z}(x, y)=\frac{1}{\pi \sigma_{x}(z) \sigma_{y}(z)} \exp \left(\frac{-x^{2}}{\sigma_{x}^{2}(z)}+\frac{-y^{2}}{\sigma_{y}^{2}(z)}\right) .
$$

The variances $\sigma_{x}$ and $\sigma_{y}$ depend on the depth coordinate $z$; they are strictly related to the NA of the excitation waveguide calculated along the horizontal and vertical directions, respectively. In the most general case, the NA of the excitation waveguide may differ from that of the collector waveguide even if both waveguides have identical cross-sections. For the excitation waveguide, in fact, the NA is highly dependent on the number of modes that are excited inside the waveguide and on how the total power is distributed among these modes. On the other hand, this distribution depends on the input fiber that is being used to couple the light into the excitation waveguide and on its mode profile.

Having defined $\rho_{z}(x, y)$, in case of isotropic scattering, we can write the radiant intensity $\mathrm{d} I_{\mathrm{s}}[\mathrm{W} / \mathrm{sr}]$ scattered from the volume element $\mathrm{d} x \mathrm{~d} y \mathrm{~d} z$ centered in point $Q(x, y, z)$ as

$$
\mathrm{d} I_{s}(x, y, z)=P_{e} T_{e} \rho_{z}(x, y) \beta N \mathrm{~d} x \mathrm{~d} y \mathrm{~d} z,
$$

where $\beta$ is the differential scattering cross-section $\left[\mathrm{m}^{2} \mathrm{sr}^{-1}\right]$ and $N$ is the number of scattering molecules per unit volume $\left[\mathrm{m}^{-3}\right]$. By multiplying (3.3) with the solid angle $\Omega$ subtended by the effective collection area at point $Q$ we obtain the fraction $\mathrm{d} P_{\mathrm{c}}$ of optical power, scattered from the infinitesimal volume $\mathrm{d} x \mathrm{~d} y \mathrm{~d} z$, that reaches the collector waveguide:

$$
\mathrm{d} P_{c}=\mathrm{d} I_{s}(x, y, z) \Omega(x, y, z) .
$$

In order to compute $\Omega$ we need to estimate the effective collection area of the waveguide, which is different from its geometrical cross-section. To estimate the collection area we consider a plane wave with unitary power density $\left(1 \mathrm{~W} / \mathrm{m}^{2}\right)$ incident on the waveguide facet at an angle $\vartheta$ with the $z$ axis and $\varphi$ with the $y$ axis (as shown in Fig. 3.3). The Fresnel reflection coefficients are taken into account for both $s$ - and $p$-polarized incident light. The effective collection area $A_{\text {eff }}$ (see Fig. 3.4) 
is then calculated numerically as the total power coupled into the TE and TM modes of the waveguide, divided by the power density of the incident plane wave. The power radiated from point $Q$ that is collected by the probe is simply estimated by multiplying $A_{\text {eff }}$ by the power density at the waveguide facet, which is a function of the distance $r=\sqrt{x^{2}+y^{2}+z^{2}}$ between $Q$ and the waveguide facet.
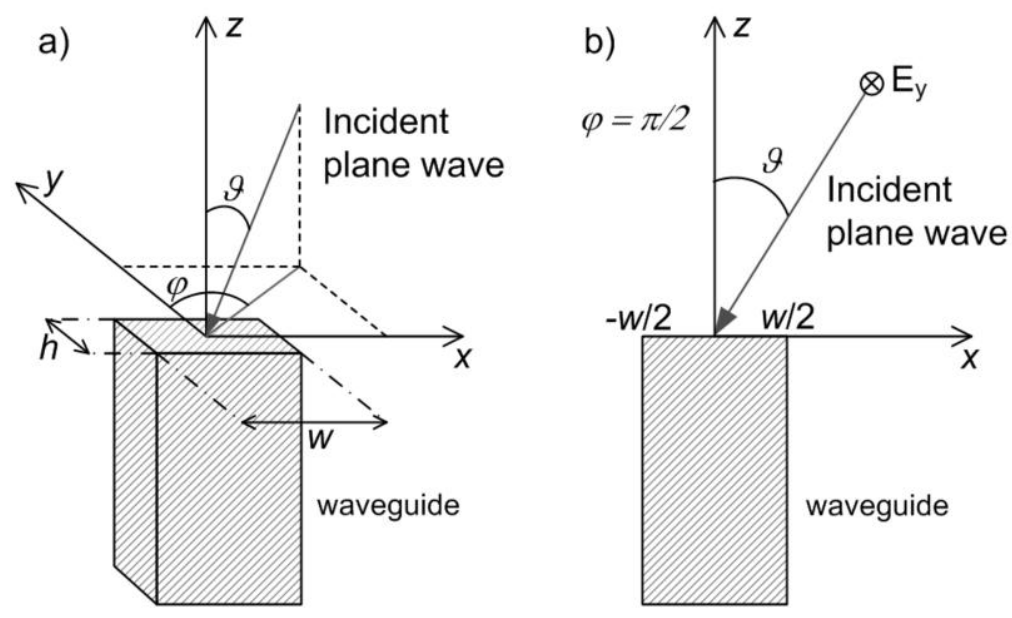

Fig 3.3 - Plane wave incident on waveguide facet, (a) from an arbitrary direction; (b) $s$ polarized plane wave incident in the X-Z plane (b).

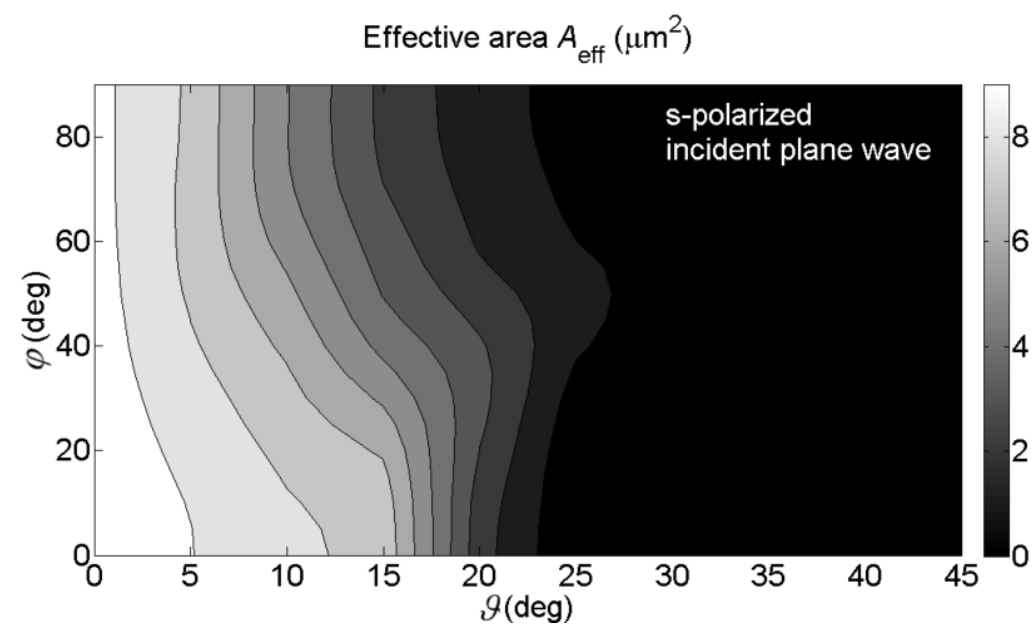

Fig 3.4 - Effective collection area $A_{\text {eff }}(\vartheta, \varphi)$ for a waveguide with cross-section $5 \mu \mathrm{m} \times$ $0.82 \mu \mathrm{m}$ and for an $s$-polarized incident plane wave.

The effective area $A_{\text {eff }}$ is a function of the incidence angles $\vartheta$ and $\varphi$, usually having a maximum for $\vartheta=0$ and approaching zero for $\vartheta$ approaching $\pi / 2$. As the angle $\varphi$ changes, there is a change in the power distribution among the TE and TM guided modes, which results in a variation in the effective area. When $\varphi=\pi / 2$, the 
incidence plane coincides with the $x-z$ plane and the electric field of an s-polarized incident plane wave would be along the y axis as shown in Fig. 3.3 (b). This field would excite only TM guided modes. On the other hand, when $\varphi=0$, an $s$-polarized incident plane wave would excite only TE propagating modes in the waveguide. By expressing the effective area as a function of the coordinates (the angles $\vartheta$ and $\varphi$ can be expressed in terms of the coordinates $x, y, z)$ of point $Q$, the solid angle $\Omega$ can be calculated as:

$$
\Omega(x, y, z)=4 \pi \frac{A_{e f f}(x, y, z)}{A_{s}(x, y, z)},
$$

where $A_{\mathrm{s}}$ is the area of the sphere centered in $Q$ and with radius $r$. It should be noted, however, that this calculation is correct only if the probe is in touch with the sample or the gap between them consists of a material with a refractive index matching that of the sample. In case neither of these two conditions is met, the solid angle will change due to refraction and its calculation becomes inefficient from a computational point of view [Oli93]. For this reason, in our model we only treat the simple case in which the material between probe and sample has the same refractive index as the sample.

Finally, combining (3.2) through (3.5), $\mathrm{d} P_{\mathrm{c}}$ can be expressed as

$$
\mathrm{d} P_{c}=\frac{P_{e} T_{e} A_{e f f}(x, y, z) \beta N}{\pi r^{2} \sigma_{x}(z) \sigma_{y}(z)} \exp \left(\frac{-x^{2}}{\sigma_{x}^{2}(z)}+\frac{-y^{2}}{\sigma_{y}^{2}(z)}\right) \mathrm{d} x \mathrm{~d} y \mathrm{~d} z .
$$

The figure of merit $S[\mathrm{sr} \cdot \mathrm{m}]$, which allows comparing different probe configurations, is defined by integrating $\mathrm{d} P_{\mathrm{c}}$ over the entire overlap volume $V$ of the excitation and the collection cones and dividing by $P_{\mathrm{e}} \beta N$, so that the quantity is independent of the sample's scattering properties and the input power, but only depends on the geometry of the probe and the overlap volume. In this way we obtain

$$
S=\iiint \frac{T_{e} A_{e f f}(x, y, z)}{\pi r^{2} \sigma_{x}(z) \sigma_{y}(z)} \exp \left(\frac{-x^{2}}{\sigma_{x}^{2}(z)}+\frac{-y^{2}}{\sigma_{y}^{2}(z)}\right) \mathrm{d} x \mathrm{~d} y \mathrm{~d} z .
$$

\subsubsection{Description of probe}

We designed and fabricated an integrated waveguide probe having the geometry shown in Fig. 3.1. The probe has one excitation waveguide and eight collector waveguides with a SiON core and silicon oxide cladding. The propagation losses in SiON waveguides are approximately $0.4 \mathrm{~dB} / \mathrm{cm}$ for the wavelengths used in our measurements (532 $\mathrm{nm}$ and $693 \mathrm{~nm}$ ) [Wör02]. The distance $d$ between the waveguides is fixed to $11 \mu \mathrm{m}$ and each waveguide's cross-section is $5 \mu \mathrm{m} \times 0.82$ $\mu \mathrm{m}$. The refractive indices of core and cladding at a wavelength of $532 \mathrm{~nm}$ have been estimated for TE polarization as 1.5321 and 1.4631, respectively. 
The NA of the excitation waveguide was measured experimentally; we briefly discuss the method, since the results are used as an input to our simulations. The measurement was performed by placing an opaque screen in front of the waveguide's output facet and a CCD camera focused on the screen. The distance between screen and camera was fixed to the focal distance of the camera. The camera was used to measure the spot size for two different distances of the screen from the waveguide facet. Each spot was approximated with a Gaussian profile and the spot size was estimated in both, horizontal and vertical directions by considering the points where the intensity decayed to $1 / \mathrm{e}^{2}$ of the maximum. The input fiber used to couple the laser light into the excitation waveguide was single-mode for the wavelength of $532 \mathrm{~nm}$.

The NA of a collector waveguide is different from that of the excitation waveguide. In order to estimate it we calculated the overlap integral between a plane wave, incident at an angle $\vartheta$ on the waveguide facet and each of the guided modes (for the particular wavelength that needs to be detected). Varying $\vartheta$ between 0 and $\pi / 2$ (see Fig. 3.3), in the horizontal $(\varphi=\pi / 2)$ and vertical $(\varphi=0)$ directions, we obtain the mode spectra of all guided modes; each mode spectrum represents the power which is coupled to the mode by a plane wave as a function of the angle of incidence (or the far-field angular distribution of the intensity radiated by that mode) [Her76]. By summing the powers coupled to each mode we obtain the total power coupled to the waveguide for a given angle of incidence. The total power $P(\vartheta)$ as a function of $\vartheta$ (varied in both horizontal and vertical directions) is shown in Fig. 3.5 for TE and TM polarizations. The collector waveguide is single-mode in the vertical direction and supports 5 TE modes (and as many TM modes) in the horizontal direction. This explains the monotonic decay found in Fig. 3.5 (b). For zero horizontal tilt only even modes couple to the waveguide. We define the acceptance angle $\vartheta_{a}$, as the angle for which the integral of $P(\vartheta)$ from 0 to $\vartheta_{a}$ is $95.5 \%{ }^{1}$ of the integral of $P(\vartheta)$ from 0 to $\pi / 2$. The measured NA values for the excitation waveguide and the calculated NA values for the collector waveguides are given in Table 3.1.

\footnotetext{
${ }^{1}$ In case of a Gaussian profile, the NA would be defined by the angle at which the collected power decays by $1 / \mathrm{e}^{2}$ with respect to the maximum. The integral of a Gaussian function between 0 and the point where it decays by $1 / \mathrm{e}^{2}$ is $95.5 \%$ of the integral between 0 and $+\infty$.
} 

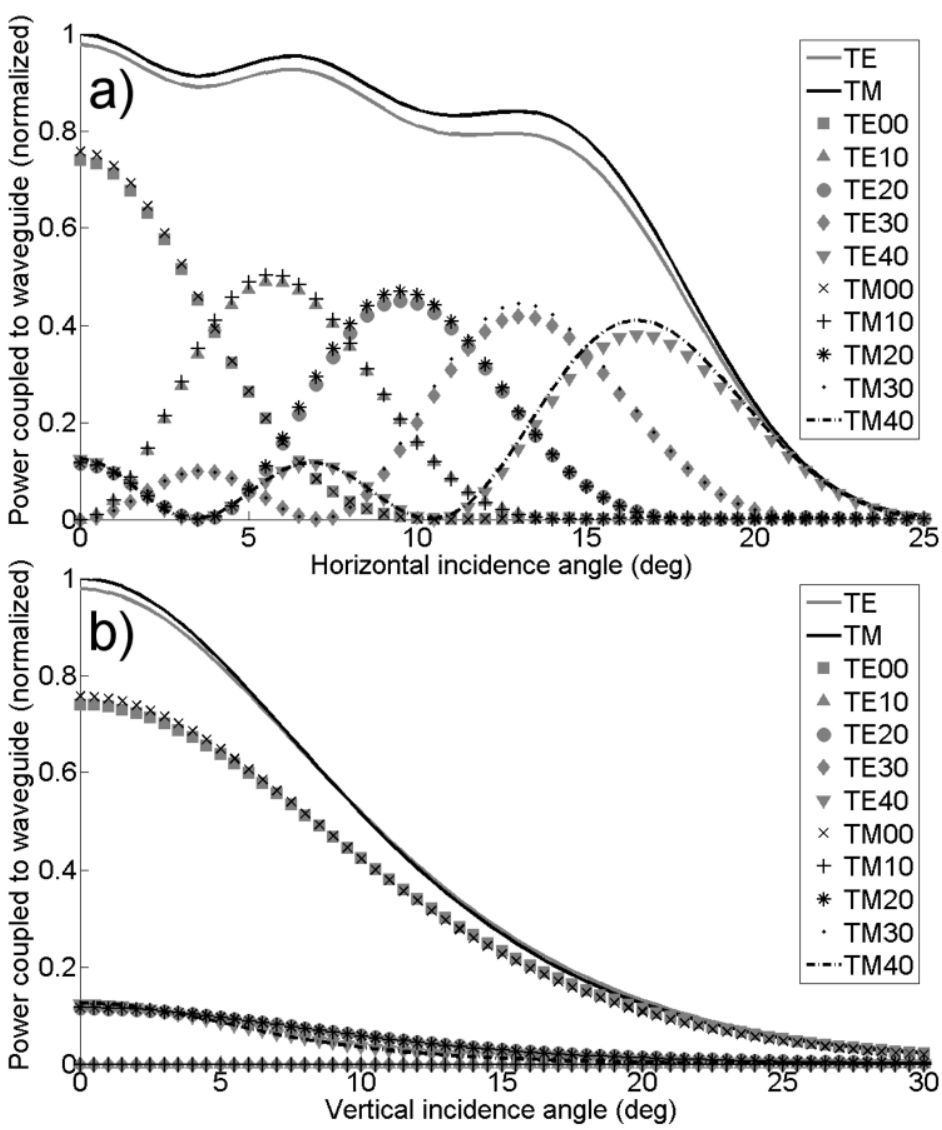

Fig 3.5 - Total power (solid lines) coupled to the collector waveguide (for TE and TM polarizations) from an incident plane wave $(\lambda=693 \mathrm{~nm})$ tilted by an angle $\vartheta$. (a) horizontal tilt (when $\varphi=\pi / 2$ ); (b) vertical tilt (when $\varphi=0$ ). The power coupled to each mode (markers and dashed lines) is shown for both horizontally and vertically tilted plane waves.

Table 3.1 - Probe parameters

\begin{tabular}{ll}
\hline$w(\mu \mathrm{m})$ & 5 \\
$h(\mu \mathrm{m})$ & 0.82 \\
$\mathrm{NA}_{\mathrm{x}}-$ excitation waveguide, $\lambda=532 \mathrm{~nm}$ & 0.060 \\
$\mathrm{NA}_{\mathrm{y}}-$ excitation waveguide, $\lambda=532 \mathrm{~nm}$ & 0.252 \\
$\mathrm{NA}_{\mathrm{x}}-$ collector waveguide, $\lambda=532 \mathrm{~nm}$ & 0.390 \\
$\mathrm{NA}_{\mathrm{y}}-$ collector waveguide, $\lambda=532 \mathrm{~nm}$ & 0.462 \\
$\mathrm{NA}_{\mathrm{x}}-$ collector waveguide, $\lambda=693 \mathrm{~nm}$ & 0.342 \\
$\mathrm{NA}_{\mathrm{y}}-$ collector waveguide, $\lambda=693 \mathrm{~nm}$ & 0.406 \\
Number of TE modes, $\lambda=532 \mathrm{~nm}$ & 11 \\
Number of TE modes, $\lambda=693 \mathrm{~nm}$ & 5 \\
\hline
\end{tabular}




\subsubsection{Simulation results}

The integral in (3.7) was evaluated numerically for the integrated probe in Table 3.1, with $d=11 \mu \mathrm{m}$, positioned in contact with the sample (i.e., $D=0$ ), for different sample thicknesses and for a similar probe with $d=6 \mu \mathrm{m}$. For comparison, we also calculated the figure of merit of three different fiber probes: a typical Raman largecore multi-mode dual-fiber probe (core diameter of $200 \mu \mathrm{m}$ ) with $d=210 \mu \mathrm{m}$, a small-core multi-mode dual-fiber probe (core diameter of $5 \mu \mathrm{m}$ ) with $d=11 \mu \mathrm{m}$ and a small-core single-mode dual-fiber probe (core diameter of $3 \mu \mathrm{m}$ ) with $d=11 \mu \mathrm{m}$. For all fiber probes a refractive index contrast of 0.01 was assumed and the distance $d$ between the excitation and collection channels was chosen such to prevent coupling of the waveguides through the evanescent tails of their modal fields. In all the simulations the excitation wavelength is $532 \mathrm{~nm}$, while the wavelength of the collected signal is $693 \mathrm{~nm}$ (as is the case in the fluorescence measurements on ruby described in the next Section).

The simulation results for these probes are shown in Fig. 3.6, in which the efficiency of each probe is displayed as a function of sample thickness. It becomes clear that, in case of thin samples (thicknesses $t$ smaller than typically $110-150 \mu \mathrm{m}$ ), integrated waveguide probes provide efficiencies exceeding those of the investigated fiber probes.

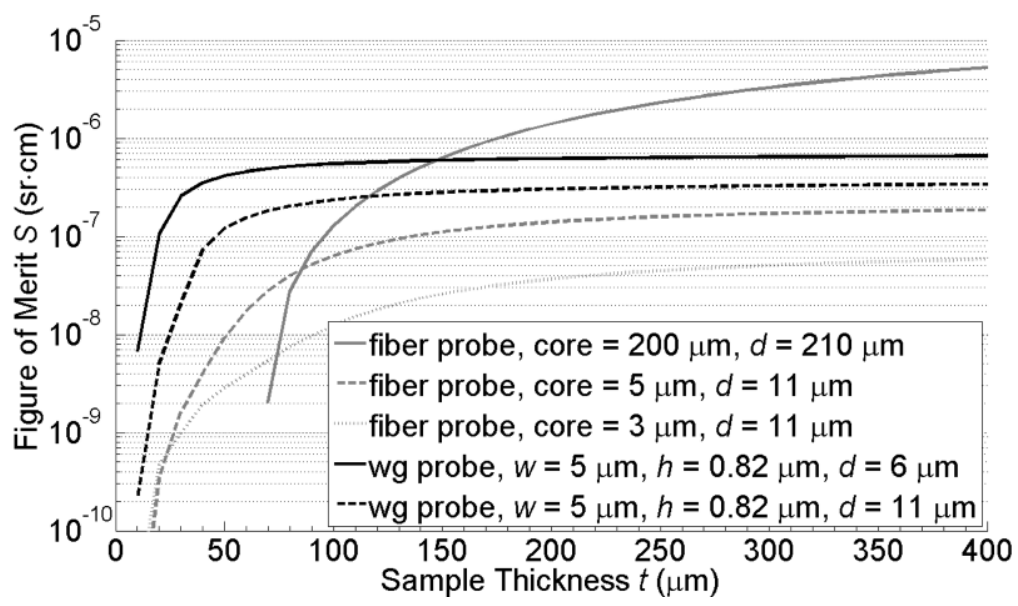

Fig 3.6 - Figure of merit $S$ as a function of the sample thickness $t$ for two integrated dualwaveguide probes with cross-sections $5 \mu \mathrm{m} \times 0.82 \mu \mathrm{m}$ and distances $d=11 \mu \mathrm{m}$ and $d=6$ $\mu \mathrm{m}$ (black lines), compared to those (gray lines) of a large-core dual-fiber probe (mathematical model in Ref. [Coo96]) with core diameter of $100 \mu \mathrm{m}$ and $d=210 \mu \mathrm{m}$ and two small-core dual-fiber probes with $d=11 \mu \mathrm{m}$ and core diameters of $5 \mu \mathrm{m}$ and $3 \mu \mathrm{m}$, respectively.

This result is relevant for our application (Raman spectroscopy of the skin) which requires the detection of Raman signals from regions within $100 \mu \mathrm{m}$ underneath the sample surface [Cas01]. Furthermore, integrated probes have higher efficiency with respect to the investigated small-core fiber probes regardless of the 
sample thickness. Although it is not problematic to fabricate micro-fiber arrays with cores of, e.g., $5 \mu \mathrm{m}$ diameter and similar distance apart from each other providing higher index contrast than that investigated in our simulations [Zha10], the advantages of integrated probes, such as reproducibility, low cost and on-chip integration with other components, make them more suitable for applications that require probing of thin samples.

Another advantage of integrated over fiber probes is their smaller collection volume and, thus, higher resolution. In order to estimate the resolution of an integrated waveguide probe we plot the power per unit thickness collected by the probe from a layer situated at a certain depth $z$ inside the sample. We vary $z$ starting from 0 up to $3 \mathrm{~mm}$. As $z$ increases, the collected power also increases until it reaches a maximum and then decreases again. This behavior can be intuitively understood by taking an infinitesimally thin layer (thickness $\mathrm{d} z$ ) at depth $z$ inside the sample and considering its intersection volume $\Delta V$ with both, excitation and collection cones (as shown in Fig. 3.2). When $z$ is equal to zero we find a null intersection volume. As the depth $z$ increases, also the intersection volume and, hence, the amount of light collected by the collector waveguide from this volume increases. However, simultaneously the solid angle $\Omega$ subtended by the collector waveguide at each point of the volume $\Delta V$ becomes smaller (inversely proportional to the square of the distance). At a certain depth the latter effect becomes predominant and the total power collected by the collector waveguide decreases with further increasing $z$. The value of $z$ at which the maximum is obtained depends on the distance $d$ between the excitation and collector waveguide. By increasing the distance $d$, the maximum is reached at increasing depth inside the sample, resulting in an increased collection volume. This is shown in Fig. 3.7, where we compare the integrated probes and fiber probes previously described.

For the large-core fiber probe, we observe a peak at $353 \mu \mathrm{m}$ depth, with a fullwidth at half-maximum (FWHM) of approximately $941 \mu \mathrm{m}$; for the small-core fiber probes with core diameters of $5 \mu \mathrm{m}$ and $3 \mu \mathrm{m}$, peaks occur at $87 \mu \mathrm{m}$ and $106 \mu \mathrm{m}$ depth, with FWHM of approximately $113 \mu \mathrm{m}$ and $191 \mu \mathrm{m}$, respectively. On the other hand, for the waveguide probes with $d=6 \mu \mathrm{m}$ and $d=11 \mu \mathrm{m}$, we observe peaks at $20.4 \mu \mathrm{m}$ and $38.6 \mu \mathrm{m}$ depth, with FWHM of $16.8 \mu \mathrm{m}$ and $31.2 \mu \mathrm{m}$, respectively.

Higher collection efficiencies can be achieved by careful design of the waveguide probes, for example by changing parameters such as waveguide width and layer thickness. By using SiON as a guiding material it is also possible to tune the refractive index of the waveguide core between 1.45 and 2.01 (as we already mentioned in Chapter 2). We carried out simulations (for $\lambda=693 \mathrm{~nm}$ ) to show how the variation of these parameters can influence the figure of merit of the probe. The results are presented in Figs. 3.8-3.10. 


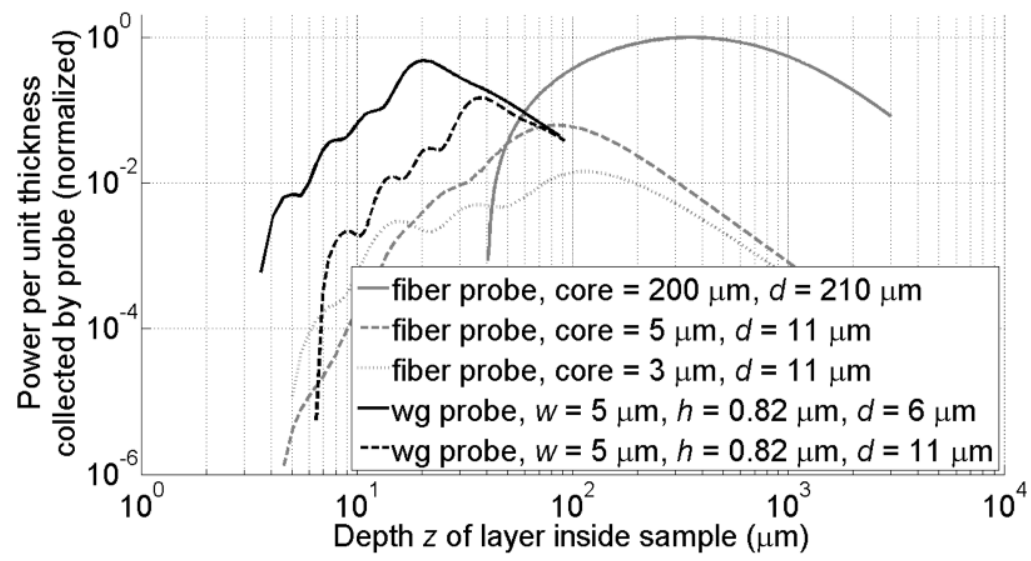

Fig 3.7 - Power per unit thickness collected by an integrated probe from a layer situated inside the sample as a function of the layer depth $z$ for two integrated dual-waveguide probes with cross-sections $5 \mu \mathrm{m} \times 0.82 \mu \mathrm{m}$ and distances $d=11 \mu \mathrm{m}$ and $d=6 \mu \mathrm{m}$ (black lines), compared to those (gray lines) of a large-core dual-fiber probe (mathematical model in Ref. [Coo96]) with core diameter of $100 \mu \mathrm{m}$ and $d=210 \mu \mathrm{m}$ and two small-core dual-fiber probes with $d=11 \mu \mathrm{m}$ and core diameters of $5 \mu \mathrm{m}$ and $3 \mu \mathrm{m}$, respectively.

Firstly, the waveguide width $w$ was varied between $1 \mu \mathrm{m}$ and $8.5 \mu \mathrm{m}$ (Fig. 3.8). As $w$ increases, the number of TE (TM) modes increases from 1 to 9, see Fig. 3.8 (a), presenting a unitary step at specific values of the waveguide width, which we refer to as cut-off widths. To estimate the collection efficiency of the waveguide we plot its effective area $\left\langle A_{\text {eff }}(\vartheta, \varphi)\right\rangle$ averaged over $0 \leq \vartheta \leq \pi / 2$ and $0 \leq \varphi \leq \pi / 2$. The average effective area increases in a step-like way, where each step corresponds to a cut-off width. We observe a decrease of the step amplitudes of the effective area as the width $w$ increases. This behavior can be understood by observing the confinement factor of the highest-order mode as a function of waveguide width, which represents the minimum of all the confinement factors of the guided modes $\Gamma_{\min }$. We plot the inverse function $1 / \Gamma_{\min }$ (in this way its variations are enhanced) after subtracting 1 for convenience of representation (see Fig. 3.8). This function presents a peak at each cut-off width (the higher the peak, the weaker the confinement of the highest-order mode). We observe that the height of the peaks decreases as the waveguide width increases, in a similar way to the height of the steps in the effective area. We conclude that the changes in the effective area occurring at the cut-off widths are larger as the confinement of the next new guided mode is weaker, i.e., this mode extends over a larger area, hence it collects more light. 

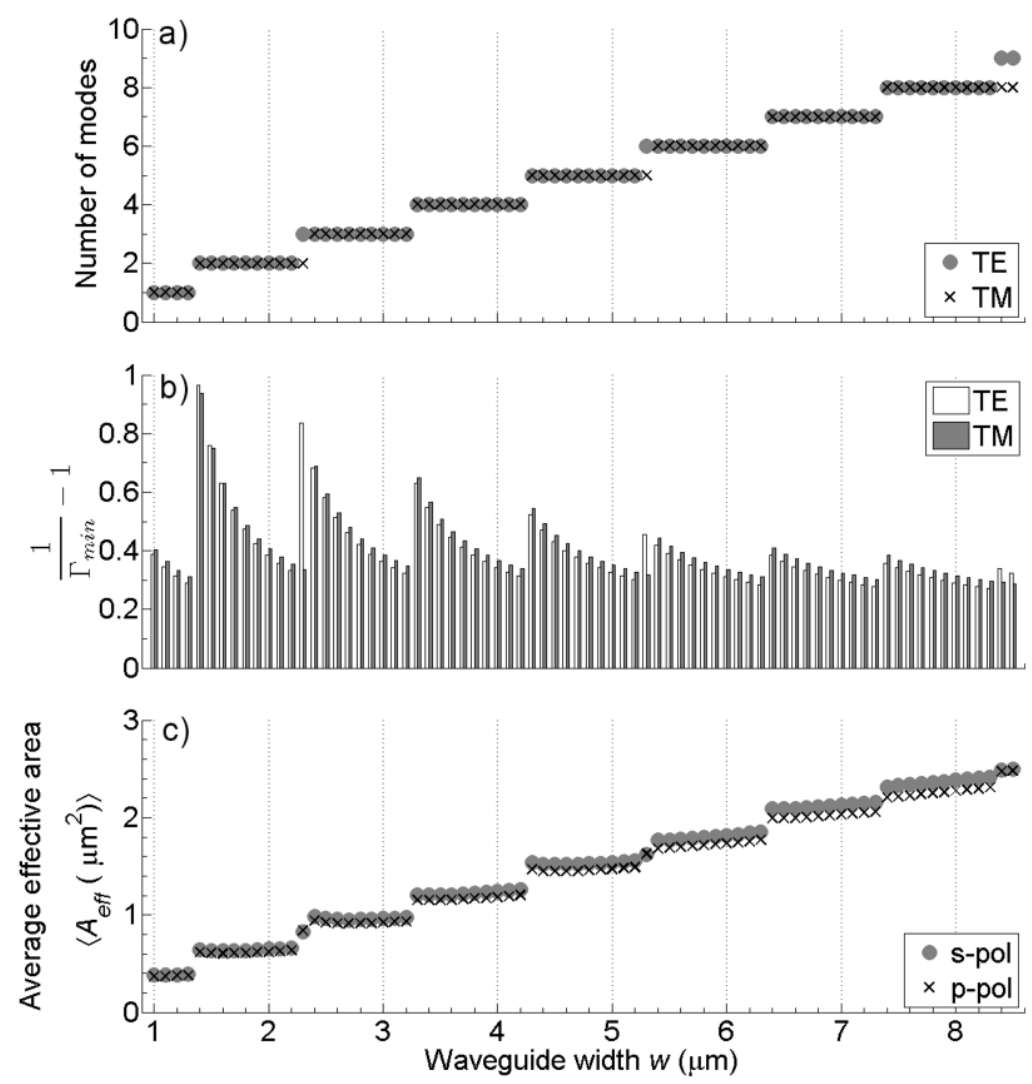

Fig 3.8 - Waveguide probe performance (for $693 \mathrm{~nm}$ ) as a function of waveguide width, at constant thickness $h=0.82 \mu \mathrm{m}$ and core index $n=1.5249$. (a) Number of modes, (b) function representing the inverse of the minimum confinement factor (apart from a constant term $=1$ ), both for TE and TM polarizations. (c) Average effective area for both $s$ - and $p$-polarized incident plane waves.

A second set of simulations was performed by varying the waveguide height between $0.25 \mu \mathrm{m}$ and $1.1 \mu \mathrm{m}$ with increments of $50 \mathrm{~nm}$ (see Fig. 3.9). In this case the number of TE (and TM) modes increases from 2 to 7. Again, we plot the function $1 / \Gamma_{\min }-1$ and the average effective area. We notice very small variations of the effective area until a height of almost $1 \mu \mathrm{m}$; then a sudden increase occurs, which corresponds to the introduction of new guided modes with low confinement factors.

In a third set of simulations the core refractive index was varied from 1.525 to 1.925 in steps of 0.01 . From the results shown in Fig. 3.10 the importance of the function $1 / \Gamma_{\min }-1$ as a tool to predict the waveguide collection efficiency becomes evident. Despite a continuous increase in the number of modes with increasing core index, the effective area presents a step increase only in two distinct points which correspond to the introduction of two sets of weakly confined modes for core index values of 1.555 and 1.715 . 
From these results, which provide an indication on how the collection efficiency of the waveguide probe behaves as its fundamental design parameters are varied, we can conclude that, in general, the probe is more efficient the larger is the area of the collector waveguide cross-section. However, there are points in which the efficiency increases in a sudden step-like manner. High efficiency can be reached by carefully choosing the waveguide parameters (width, height and refractive index contrast) in order to minimize the confinement of the highest order mode. Under certain circumstances the gain in efficiency may be significant with just a fractional increase in one of these parameters. For example, the jump in refractive index (in case of the waveguide geometry examined in Fig. 3.10) between 1.705 and 1.715 would bring an increase in efficiency of approximately 20\%; likewise, a change in height (in case of the waveguide geometry examined in Fig. 3.9) between $0.95 \mu \mathrm{m}$ and $1.1 \mu \mathrm{m}$ would result in an increase of approximately $33 \%$ in efficiency.
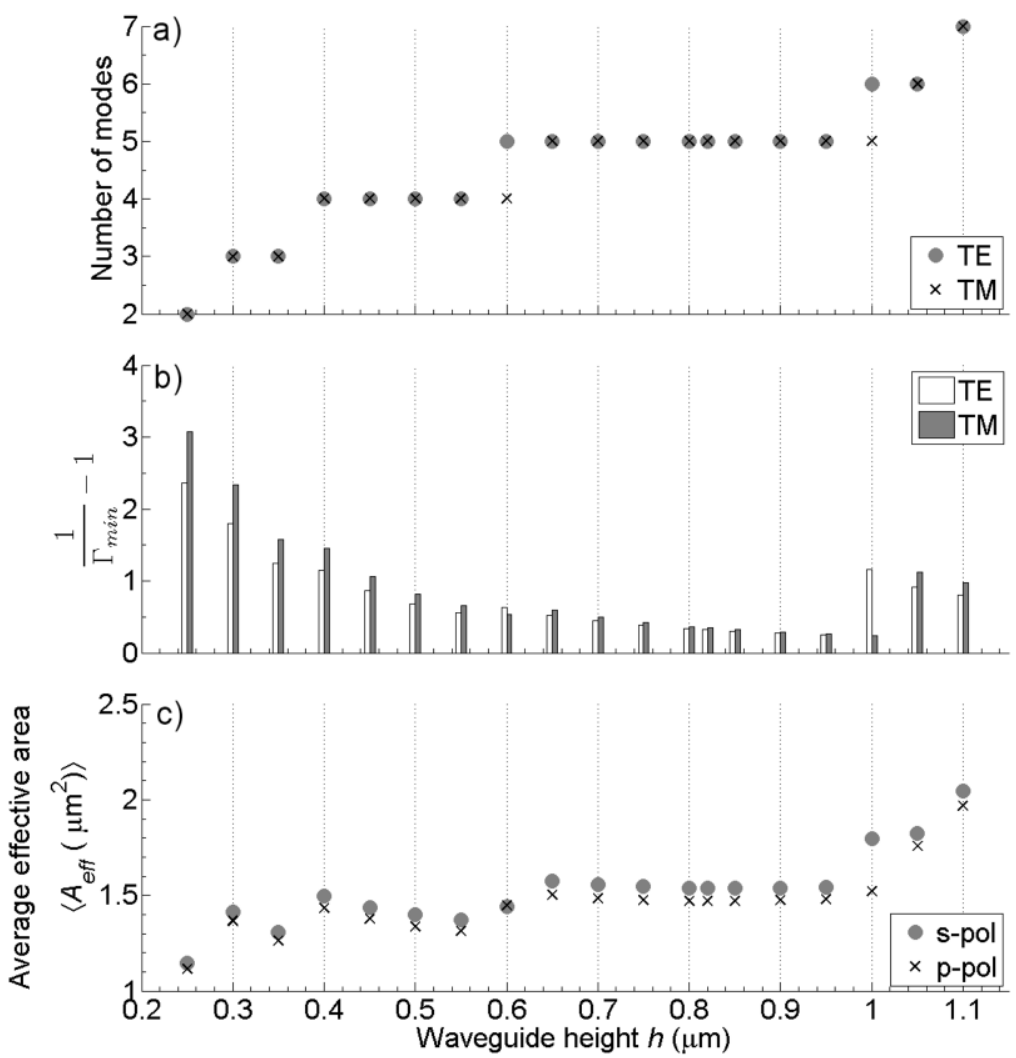

Fig 3.9 - Waveguide probe performance (for $693 \mathrm{~nm}$ ) as a function of waveguide height, for constant width $w=5 \mu \mathrm{m}$ and core index $n=1.5249$. (a) Number of modes, (b) function representing the inverse of the minimum confinement factor (apart from a constant term $=1$ ), both for TE and TM polarizations. (c) Average effective area for both $s$ - and $p$-polarized incident plane waves. 

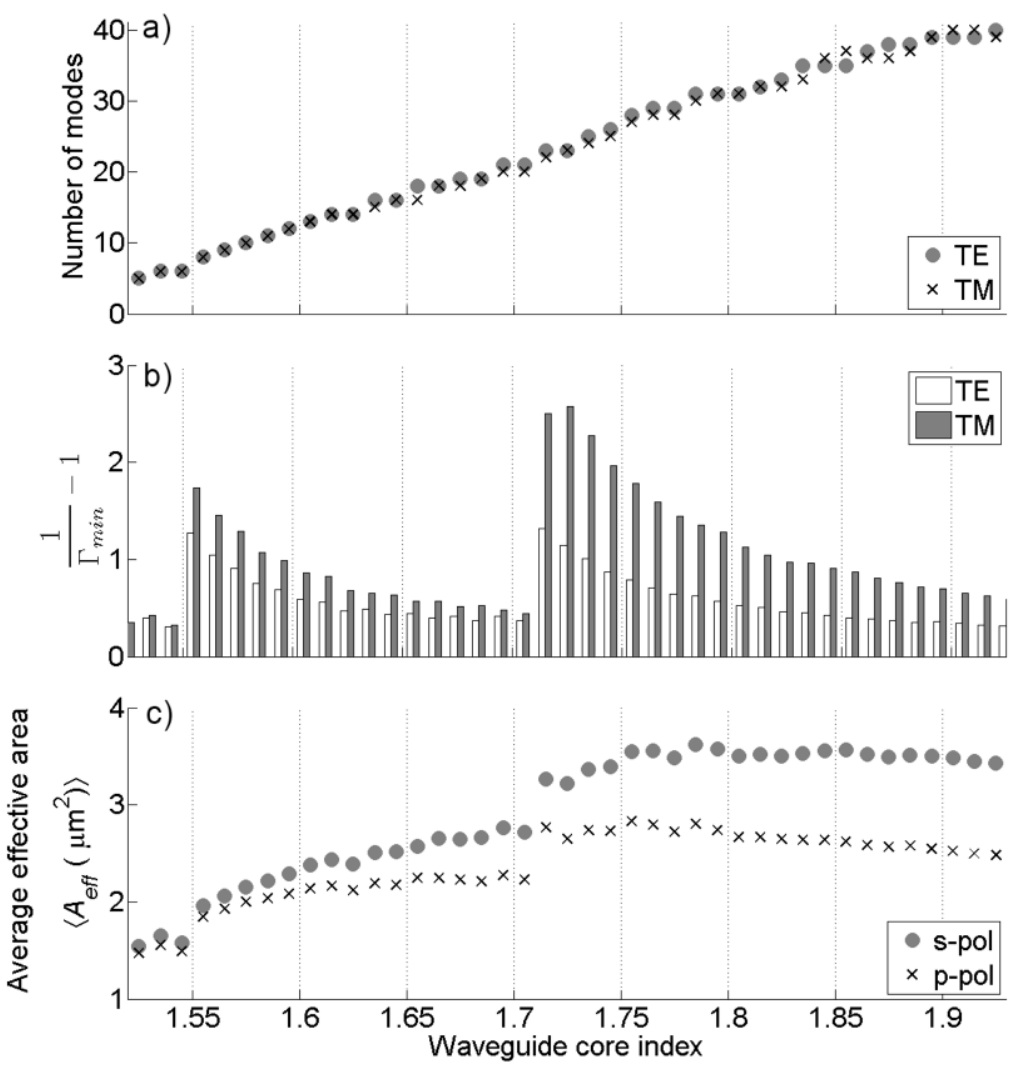

Fig 3.10 - Waveguide probe performance (for $693 \mathrm{~nm}$ ) as a function of core refractive index, for constant width $\mathrm{w}=5 \mu \mathrm{m}$ and height $\mathrm{h}=0.82 \mu \mathrm{m}$. (a) Number of modes, (b) function representing the inverse of the minimum confinement factor (apart from constant term $=1$ ), both for TE and TM polarizations. (c) Average effective area for both $s$ - and $p$ polarized incident plane waves.

\subsubsection{Experimental results}

In order to validate the analytical model, introduced in Section 3.2.1, we carried out fluorescence measurements on a ruby rod. The choice for ruby was made because the fluorescent signal in the red spectral region is very strong and can easily be detected and distinguished from possible cross-talk of the excitation light. The measurements were performed using the setup shown in Fig. 3.11 and the integrated waveguide probe introduced in Fig. 3.1. A picture of the ruby rod excited with green light is shown in Fig. 3.12. 


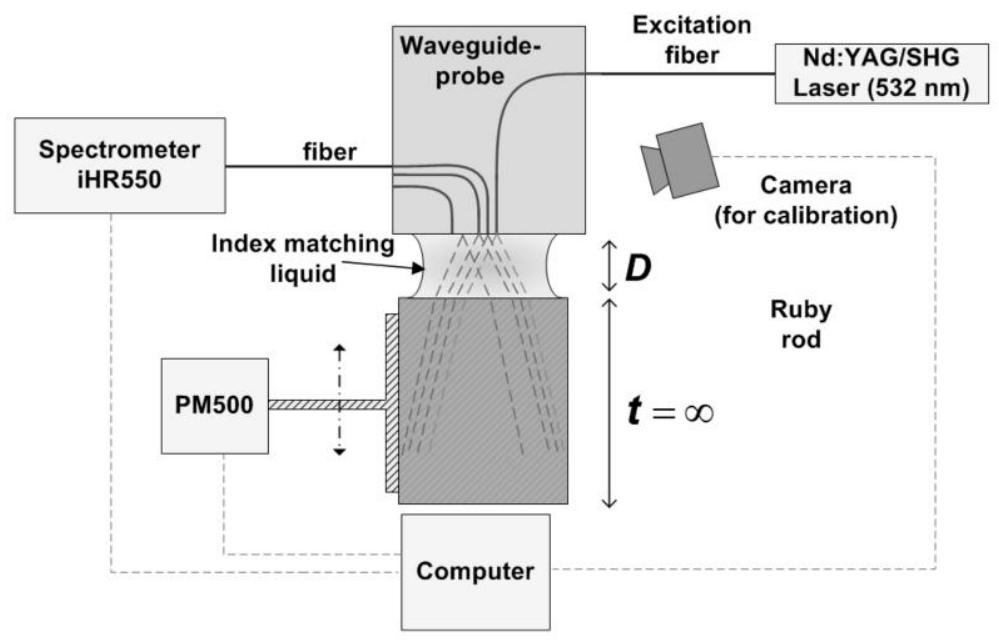

Fig 3.11 - Setup for the measurement of fluorescence from a ruby rod. The PM500 is a high-precision motion controller which allows movement of the ruby rod at different distances from the waveguide probe.

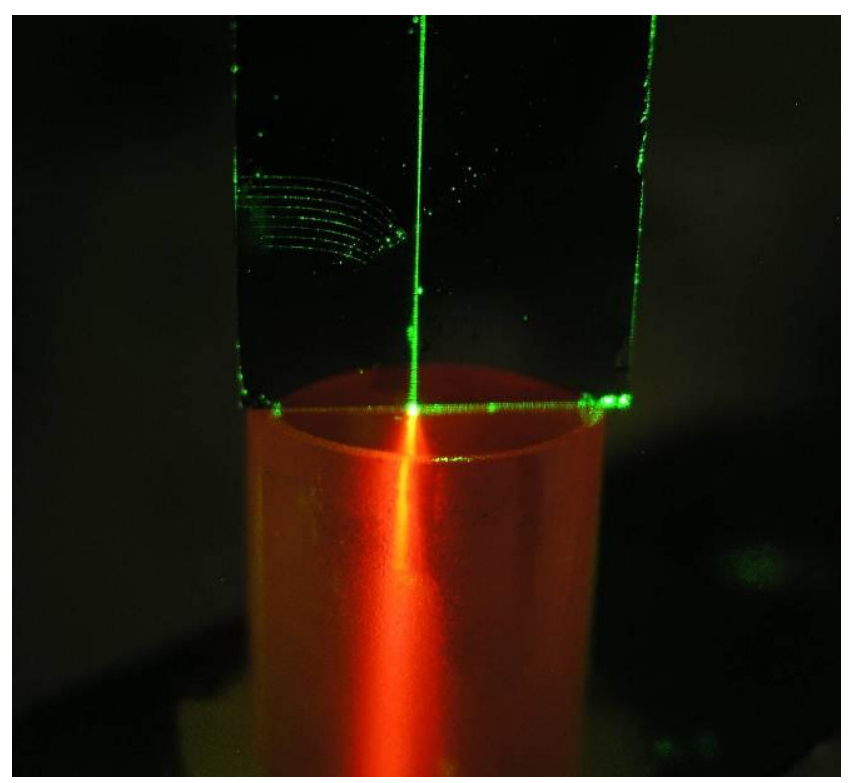

Fig 3.12 - Picture of the probe chip in contact with the ruby rod during measurement preparation.

The 10-cm-long ruby rod was attached to a Newport PM500 motion controller which allowed for a stable position at variable distance from the chip with nanometer precision. To improve the collection of fluorescent light, we used indexmatching liquid with a refractive index of 1.65 between the ruby rod and the chip. Light from a Nd:YAG/SHG laser source at $532 \mathrm{~nm}$ was coupled into the excitation waveguide. The coupling efficiency was only $18 \%$, because during the design of the 
probe no efforts were made to optimize this parameter. $120 \mu \mathrm{W}$ of power at $532 \mathrm{~nm}$ was incident on the Ruby rod. A small fraction of the fluorescence generated inside the ruby was captured by the eight collector waveguides. We performed a total of eight measurements to determine the fluorescence power captured by each collector waveguide. During the measurement, the waveguide that was being measured was coupled to a multimode 50- $\mu \mathrm{m}$-core fiber connected to a Horiba iHR550 spectrometer used for measuring the fluorescence at the ruby emission lines of 692.9 $\mathrm{nm}$ and $694.3 \mathrm{~nm}$. To ensure the same coupling between the fiber and all the output waveguides for the different measurements a calibration step was necessary. For the calibration, light from a He-Ne laser at $633 \mathrm{~nm}$ was coupled through the output fiber into the collector waveguide; the red light entering the ruby rod was then measured by use of a detector positioned at the far end of the ruby rod and the alignment between the fiber and the waveguide was adjusted until a certain signal level was reached on the detector. The same was done for all the collector waveguides that were to be measured, thereby reaching the same signal levels within a few percent. This procedure ensured that the output fiber-to-chip coupling was the same during all measurements and, therefore, no additional correction was required.

With the ruby rod positioned in contact with the chip, fluorescence measurements were separately carried out on each of the eight collector waveguides. The comparison between the experimental results and the simulation is shown in Fig. 3.13 (a). The simulation curve was obtained using the analytical model discussed previously in Section 3.2.1. One of the approximations made in the model is that of a non-absorbing medium. The absorption of ruby can be neglected for the wavelength range of interest around the $R$ lines [Mai61]. For the excitation light at $532 \mathrm{~nm}$ ruby exhibits absorption coefficients $\alpha$ of $0.12 \mathrm{~mm}^{-1}$ and $0.18 \mathrm{~mm}^{-1}$ for the incident electric field being normal or parallel to the $c$ axis of ruby, respectively [Cro66]. Therefore, we included the absorption of the excitation light (nonpolarized) in the model by multiplying (1) by $\exp (-\alpha z)$ and taking for $\alpha$ an intermediate value of $0.15 \mathrm{~mm}^{-1}$. Since the simulation of very thick layers requires a huge computational effort (of several days duration), we limited the simulations to a thickness of $3 \mathrm{~mm}$. For such thin slices no significant change was observed in the simulated curves after introducing the absorption for the excitation wavelength in the model. Simulating a larger sample thickness does not significantly change the results because, as shown in Figs. 3.6 and 3.7, the waveguide probe collects most of the light from the first few hundred $\mu \mathrm{m}$ inside the sample. Therefore, the light collected from regions deeper than $3 \mathrm{~mm}$ inside the sample can be safely neglected.

A second measurement was carried out by fixing $d$ to $11 \mu \mathrm{m}$ and measuring the power collected by the first collector waveguide of the probe at different distances of the ruby rod from the probe. A total of twelve measurements were performed by varying the distance $D$ between $25 \mu \mathrm{m}$ and $700 \mu \mathrm{m}$. The measurements compared to the simulation results are shown in Fig. 3.13 (b). In both, Figs. 3.13 (a) and (b), the comparison between measurements and simulations is achieved by normalizing each curve to its maximum value. 
The reasonable agreement between simulation and experiment validates our analytical model of integrated waveguide probes.
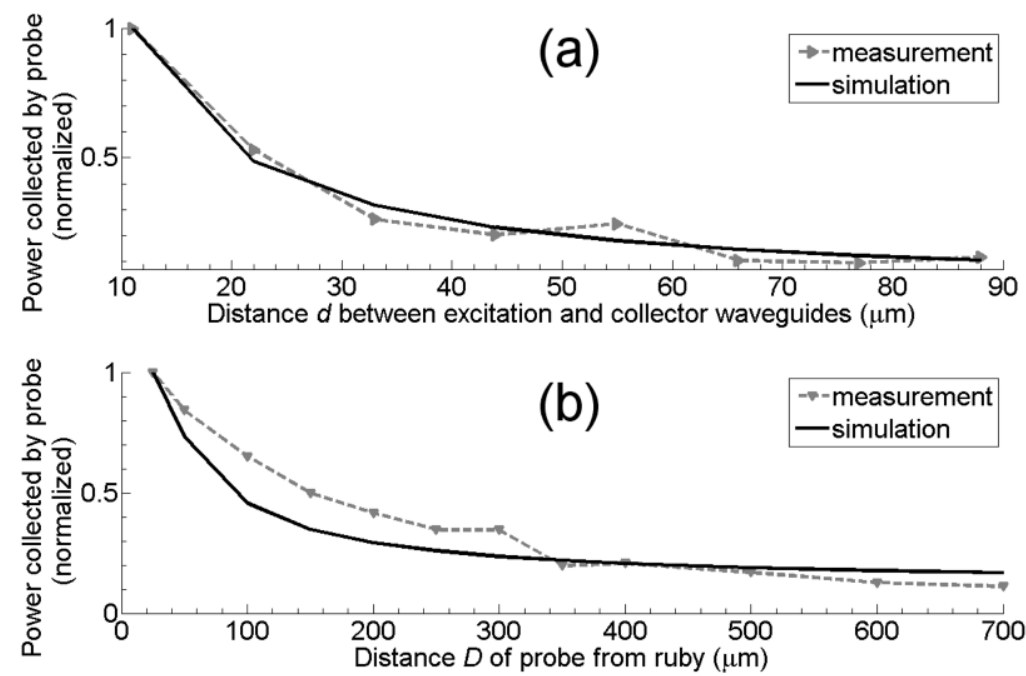

Fig 3.13 - a) Fluorescence measured by a waveguide probe as a function of the distance $d$ between excitation and collector waveguides; b) Fluorescence measured by a dualwaveguide probe with $d=11 \mu \mathrm{m}$ as a function of the distance $D$ between the probe and the ruby.

\subsection{Integrated waveguide probes for backscattered light collection from highly scattering media}

A highly scattering medium is a medium in which the distance between two subsequent scattering events is smaller than the physical dimensions of the medium so that the photons launched into the medium experience multiple scattering events before exiting from the medium (eventually reaching the detector). Different methods can be used to model photon transport through highly scattering media, as for example approaches that make use of the diffusion approximation [Ish77] or, as considered in this Chapter, Monte-Carlo simulations [Wan95, Bin06 ${ }_{\mathrm{a}, \mathrm{b}}$, Zol06].

\subsubsection{Monte-Carlo modeling of light propagation in turbid media}

We developed a Monte-Carlo model, based on the work of Wang et al. [Wan95], in which an infinitely narrow beam of light is incident on a semi-infinite multi-layered medium. A fraction of the incident photons is reflected from the surface of the medium, while the rest is absorbed or backscattered through the surface. All the photons that propagate outwards from the surface are considered as output photons: they are backscattered photons as well as photons that have been reflected from the surface. The two kinds of output photons can be easily distinguished by the program and each output photon is saved into an array that holds the position, the propagation angle relative to the $z$ axis, the optical depth (the depth at which the output photon 
encountered the last scattering center) and the penetration depth (deepest point inside the medium reached by the output photon), as well as the path length travelled by the photon packet. The density of photons that reach the surface after multiple scattering events and their propagation direction when crossing the upper interface is used to estimate the collection efficiency of the integrated optical probe.

While the optical depth is an important parameter, since it tells us from which depth inside the medium we are collecting information, the penetration depth is also important, since it can help us determine an optimum thickness for the simulated medium; in fact, one could think of simply ignoring photons that travel too deep inside the medium since these are more likely to be absorbed than to be backscattered through the surface. By ignoring these photons (limiting the depth of the medium) one could save a considerable amount of computation time. A flow chart of the Monte-Carlo program is shown in Fig. 3.14.

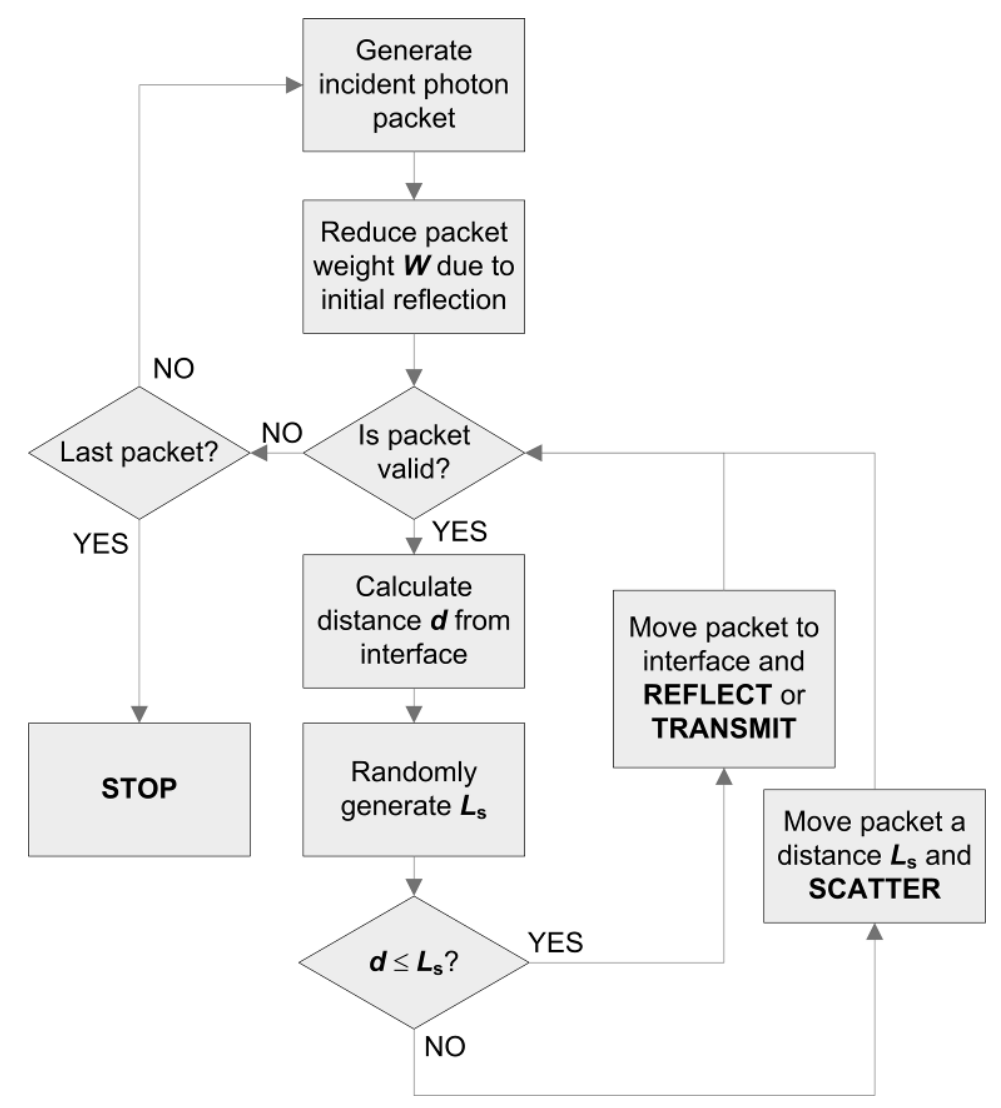

Fig. 3.14 - Monte-Carlo program flow chart, where $W$ is the photon packet weight, $L_{\mathrm{s}}$ is the distance the photon packet must travel in the medium before reaching a scattering center and $d$ is the distance the photon packet must travel before reaching the closest interface between two media. 
In the simulation we consider the light entering the medium composed of $N$ photon packets of weight $W$. One of the differences of our program with respect to that in [Wan95] is that we consider each packet entering the medium under an angle instead of at normal incidence. The angle is chosen using a random variable uniformly distributed within the angular aperture of the excitation waveguide. Once the photon packet is generated, its weight is reduced by an amount proportional to the reflection coefficient at the upper interface (which depends on the angle of incidence). At this point the packet is checked to see whether it is still valid; that is when the weight $W$ is still above a predefined threshold and the packet is not exiting the medium or has not propagated too deep into the medium (in general at the beginning of the loop all the packets are valid).

Each time the packet is successfully validated, two quantities are calculated: the distance $d$ that the packet must travel along its propagation direction to reach the closest interface between two media (in general the medium is considered made out of multiple layers) and the distance $L_{\mathrm{s}}$ that the packet must travel to reach a scattering point. While $d$ is calculated based on simple geometrical considerations, $L_{\mathrm{s}}$ is computed based on the scattering probability which depends, as will be discussed later, on the scattering coefficient of the medium.

The photon packet is then processed depending on the values of the two distances $d$ and $L_{\mathrm{s}}$; for example, if $d$ is smaller than $L_{\mathrm{s}}$, the photon packet will reach the interface before reaching a scattering center. At the interface it will be either reflected or transmitted (explained later). If $L_{\mathrm{s}}$ is the smallest distance, then the packet will first reach a scattering center, where the propagation direction of the packet is changed randomly based on the anisotropy factor $g$ of the medium. Every time the photon packet is moved by a distance $x$ its weight $W$ (initially set to 1 ) is decreased by a quantity given by $1-\exp \left(-\mu_{\mathrm{a}} x\right)$, where $\mu_{\mathrm{a}}$ is the absorption coefficient of the medium.

The distance between scattering events is defined by a random variable $L_{\mathrm{s}}$ which has associated the probability density function $p\left(L_{\mathrm{s}}\right)=\mu_{\mathrm{s}} \exp \left(-\mu_{\mathrm{s}} L_{\mathrm{s}}\right)$, as deduced from Beer's law. At each loop of the Monte-Carlo program the random variable $L_{\mathrm{s}}$ is computed by making use of another random variable $\xi$, provided by the built-in random number generator in Matlab, which is uniformly distributed in the interval $[0,1]$. In order to calculate calculating $L_{\mathrm{s}}$ once obtained $\xi$ we try to find function $f(\xi)$ that maps $\xi$ to $L_{\mathrm{s}}$. This is done considering the cumulative distribution function (CDF) of $\xi$, which is defined as:

$$
F_{\xi}(x)=P[\xi \leq x]=\left\{\begin{array}{cc}
0 & x \leq 0 \\
x & 0<x \leq 1 . \\
1 & x>1
\end{array} .\right.
$$


In (3.8) $P[\xi \leq x]$ indicates the probability that $\xi$ is smaller than or equal to $x$. The function $f(\xi)$ must be chosen such that the following equality holds:

$$
P\left[f(0)<L_{s} \leq f(x)\right]=P[0<\xi \leq x],
$$

where $x$ is arbitrarily chosen in the interval [0,1]. The equality (3.9), by imposing $f(0)=0$ and using (3.8), can be expressed as:

$$
F_{L s}(f(x))=F_{\xi}(x) \Rightarrow \int_{0}^{f(x)} p\left(L_{s}\right) d L_{s}=x,
$$

which can be written as:

$$
\int_{0}^{L_{s}} \mu_{s} e^{-\mu_{s} L} d L=1-e^{-\mu_{s} L_{s}}=\xi .
$$

This leads to the following expression for $L_{\mathrm{s}}$ :

$$
L_{s}=\frac{-\ln (1-\xi)}{\mu_{s}}=\frac{-\ln \xi}{\mu_{s}}
$$

Expression (3.12) enables us to generate the random variable $L_{\mathrm{s}}$ at each loop by simply making use of the built-in random number generator in Matlab, which generates random numbers uniformly distributed in $[0,1]$. In expression $(3.12) \xi$ is a random number uniformly distributed in the interval $[0,1]$ and therefore so is $1-\xi$. For this reason, from a statistical point of view $-\ln (1-\xi) / \mu_{\mathrm{s}}$ is equivalent to $-\ln (\xi) / \mu_{\mathrm{s}}$. When very large numbers of photons are fed into the Monte-Carlo algorithm and $L_{\mathrm{s}}$ is calculated many times in a loop, the latter expression is more convenient to use, since it requires one arithmetic operation less.

When a photon reaches the boundary between two media, it can be reflected or transmitted. The probability of reflection is given by the Fresnel reflection coefficient $R$, while the probability of transmission is $T=1-R$.

$$
R\left(\vartheta_{i}\right)=\frac{1}{2}\left[\frac{\sin ^{2}\left(\vartheta_{i}-\vartheta_{t}\right)}{\sin ^{2}\left(\vartheta_{i}+\vartheta_{t}\right)}+\frac{\tan ^{2}\left(\vartheta_{i}-\vartheta_{t}\right)}{\tan ^{2}\left(\vartheta_{i}+\vartheta_{t}\right)}\right],
$$

where $\vartheta_{i}$ is the angle of incidence and $\vartheta_{t}$ is the angle of transmission. In case the second medium refractive index is smaller than the first, total internal reflection can occur if $\vartheta_{i}>\vartheta_{c}=\arcsin \left(n_{2} / n_{1}\right)$. In this case $R=1$ and $T=0$. In case of reflection, only the propagation direction along $z$ is inverted and in case of transmission the propagation angle $\vartheta$ is changed to $\vartheta_{t}$. We choose whether to reflect or transmit a photon packet by generating a random number $\xi$ uniformly distributed between 0 and 1 , and comparing it with $R$. In case $R$ is greater than $\xi$ the photon is reflected, otherwise transmitted. 
When a packet reaches a scattering center its propagation direction is changed in a random way: while the azimuthal angle $\varphi$ is distributed uniformly in the interval $[0,2 \pi]$, the polar deflection angle $\vartheta$ (defined as the deviation of the ray from the forward direction) follows a non-uniform probability distribution between 0 and $\pi$. In turbid media, such as skin, the probability distribution function of the angle $\vartheta$ is well approximated by the Henyey-Greenstein (HG) phase function [Zol08, Bin06 ${ }_{\mathrm{a}, \mathrm{b}}$ ] given by:

$$
p_{H G}(\vartheta)=\frac{1}{2} \frac{\left(1-g^{2}\right) \sin \vartheta}{\left(1+g^{2}-2 g \cos \vartheta\right)^{3 / 2}},
$$

where $g$ is the anisotropy factor. For $g=0$ we have an isotropic distribution of the scattered light, for $g=1$ all the radiation is thrown forward; while if $g=-1$ we have pure backward scattering. The original Henyey-Greenstein phase function, proposed in 1941, was used to characterize interstellar scattering [Hen41].

It is possible to simulate the random event that follows the probability distribution of $\vartheta$ by choosing a random number $\xi$ uniformly distributed in the interval $[0,1]$ and imposing:

$$
\xi=\int_{0}^{\vartheta} p_{H G}\left(\vartheta^{\prime}\right) d \vartheta^{\prime} .
$$

Equation (3.15) can only be solved numerically (in terms of the angle Э). However, as explained in [Wit77], it is possible to analytically compute the quantity $\cos (\vartheta):$

$$
\cos \vartheta= \begin{cases}\frac{1}{2 g}\left\{1+g^{2}-\left[\frac{1-g^{2}}{1-g+2 g \xi}\right]\right\} & g \neq 0 \\ 2 \xi-1 & g=0\end{cases}
$$

This method has been used in [Pra89, Wan95, Chi07] and is commonly used in modeling light propagation in tissue. The set of equations (3.16) enables us to directly compute the new direction of the photon packet after a scattering event, in both cases of anisotropic $(g \neq 0)$ and isotropic $(g=0)$ scattering media, by making use of a random variable $\xi$ with uniform probability distribution in the interval $[0$, $1]$. 


\subsubsection{Optical properties of the scattering medium}

As a scattering medium in both, experiments and simulations we used a water suspension of latex spheres with diameter of $88 \mathrm{~nm}$ and a concentration of $69.93 \times 10^{18} \mathrm{~m}^{-3}$. This type of medium is chosen for the well-known scattering properties of such spheres. In order to calculate the scattering coefficient $\mu_{\mathrm{s}}\left[\mathrm{mm}^{-1}\right]$ of the suspension we used the following formulas for Mie scattering:

$$
\begin{gathered}
Q_{s c a}=\frac{2}{x^{2}} \sum_{n=1}^{N}(2 n+1)\left(\left|a_{n}\right|^{2}+\left|b_{n}\right|^{2}\right) \\
C_{s c a}=Q_{s c a} / G \\
g=\frac{4}{x^{2} Q_{s c a}} \sum_{n=1}^{N}\left[\frac{n(n+2)}{n+1} \operatorname{Re}\left(a_{n} a_{n+1}^{*}+b_{n} b_{n+1}^{*}\right)+\frac{2 n+1}{n(n+1)} \operatorname{Re}\left(a_{n} b_{n}^{*}\right)\right] \\
\mu_{s}=C_{s c a} c_{s}
\end{gathered}
$$

where, $Q_{\text {sca }}$ is the scattering efficiency, $C_{\text {sca }}$ is the scattering cross-section, $G$ is the particle's geometrical cross-section, $g$ is the anisotropy factor, $x$ is a size parameter given by the sphere's circumference divided by the wavelength, $N$ is the maximum number of terms after which the series can be truncated and is chosen equal to $x+4 x^{1 / 3}+2$ [Boh98], $a_{\mathrm{n}}$ and $b_{\mathrm{n}}$ are the complex-valued Mie coefficients (with the superscript asterisk ${ }^{*}$ we indicate the complex conjugate) which depend on $x$ and the refractive index contrast between spheres and the surrounding medium (in this case water), $\mu_{\mathrm{s}}$ is the scattering coefficient and $c_{\mathrm{s}}$ is the particle concentration in the suspension. The Mie coefficients are expressed in terms of spherical Bessel functions and are calculated using the code proposed in [Boh98]. The calculated values of the scattering coefficient and the anisotropy factor for the suspension at the excitation wavelength of $532 \mathrm{~nm}$ are shown in Table 3.2. This wavelength is the same as used in the experiment described in the next Section.

Table 3.2 - Optical properties of scattering medium

\begin{tabular}{ll}
\hline Diameter $(\mathrm{nm})$ & 88 \\
Refractive index of suspension & 1.339 \\
Refractive index of water $(\lambda=532 \mathrm{~nm})$ & 1.336 \\
Refractive index of latex spheres $(\lambda=532 \mathrm{~nm})$ & 1.583 \\
Weight/volume concentration $\left(\mathrm{kg} \cdot \mathrm{m}^{-3}\right)$ & 26.2 \\
Concentration $c_{\mathrm{s}}\left(\mathrm{m}^{-3}\right)$ & $69.93 \times 10^{18}$ \\
Scattering coefficient $\mu_{s}\left(\mathrm{~mm}^{-1}\right)$ & 3.315 \\
Absorption coefficient $\mu_{a}\left(\mathrm{~mm}^{-1}\right)$ & $3.8 \times 10^{-5}$ \\
Anisotropy factor $(0<g<1)$ & 0.0832 \\
\hline
\end{tabular}

The refractive index of the latex spheres has been set to 1.583 at $532 \mathrm{~nm}$ [Ma03]; while that of water is 1.336 [Sch90]. The absorption coefficient $\mu_{\mathrm{a}}$ of the suspension has been set equal to the absorption coefficient of water $\sim 3.8 \times 10^{-5}$ 56 
[Smi81] at a wavelength of $532 \mathrm{~nm}$, since the latex spheres do not contribute significantly to the absorption [Qui95, Pas05].

\subsubsection{Simulation results}

The Monte-Carlo algorithm is executed multiple times using four million photons in each run. The optical properties of the suspension used as input parameters for the program are taken from Table 3.2. After 100 runs we obtain the density of backscattered photons as a function of lateral distance $d$ from the excitation point shown in Fig. 3.15 (solid line).

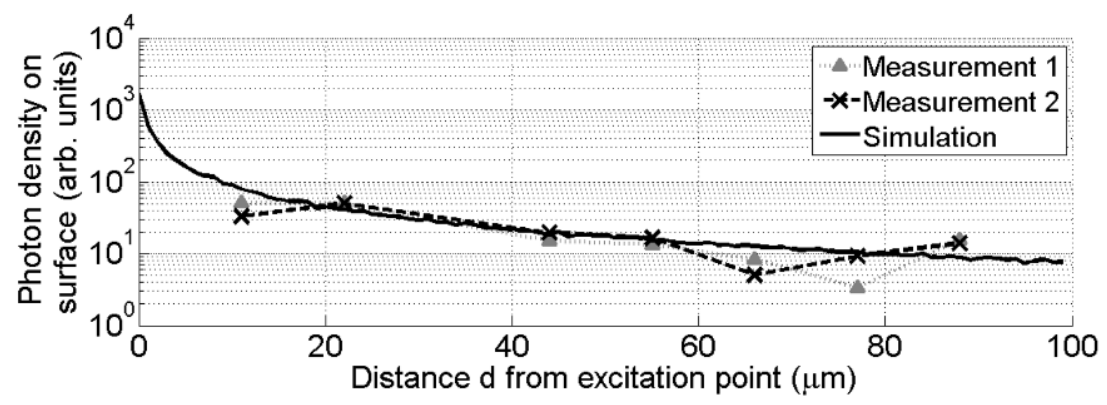

Fig. 3.15 - Experimental and simulated photon density distribution at the surface of the liquid sample as a function of distance from the excitation waveguide (the data points at $d$ $=33 \mu \mathrm{m}$ are missing due to a damaged facet of the corresponding collector waveguide).

By saving the location of the last scattering event of each collected photon we find the regions inside the sample from which photons are collected with the highest probability. When considering a probe configuration as the one shown in Fig. 3.1, with one excitation waveguide and 8 collector waveguides we find collection regions with full width at half maximum (FWHM) of $15 \mu \mathrm{m}$ and $135 \mu \mathrm{m}$ centered at $5 \mu \mathrm{m}$ and $45 \mu \mathrm{m}$ depth, for the inner and outermost waveguides, respectively. In Fig 3.16 we display the angular distribution of backscattered photons, i.e., the number of photons per unit exit angle and unit area, at the surface position of each of the 8 collector waveguides of our probe (see Fig 3.1). Only rays that exit with angles below the limit given by the NA of the collector waveguides are captured. In Fig. 3.16 the maximum exit angle is 63.2 degrees, above which total internal reflection at the chip-liquid interface keeps the backscattered light inside the sample. The ripple in the curves is due to numerical noise originating from the pseudo-random number generator used to generate the random trajectories of the photon packets and to the limited number of photons (100 runs with $4 \times 10^{6}$ photons each) used in the simulation. 


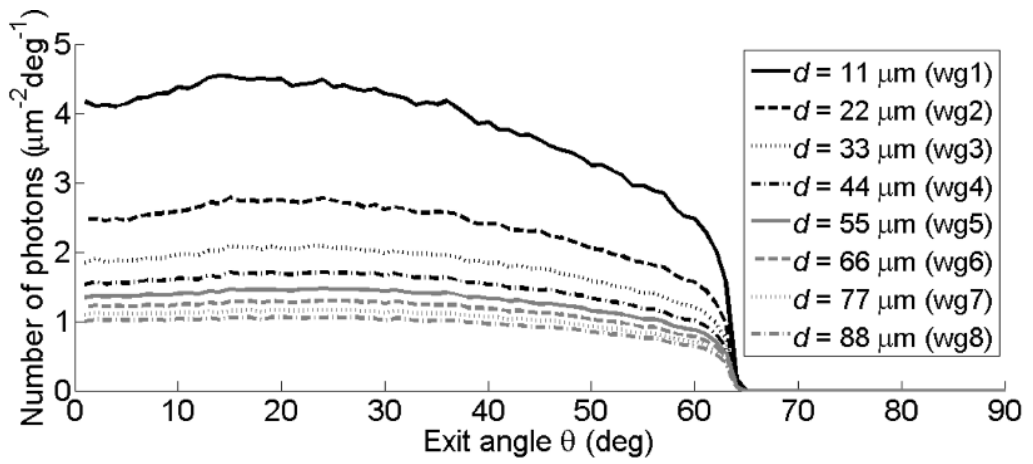

Fig. 3.16 - Calculated angular distribution of backscattered photons. Each curve represents the angular distribution in a region located around one of the collector waveguides. The distance of each waveguide (numbered from 1 to 8 ) from the excitation waveguide is indicated in the legend.

\subsubsection{Experimental results}

The integrated waveguide probe is tested by performing backscattering measurements on the suspension of latex spheres with the optical properties as given in Table 3.2. The experimental setup for the measurements is shown in Fig. 3.17. Light from a frequency-doubled Nd:YAG laser at $532 \mathrm{~nm}$ is coupled into the excitation waveguide and enters the sample droplet, which is in contact with the chip facet. In these experiments $120 \mu \mathrm{W}$ of excitation power is incident on the sample. The fraction of backscattered light captured by the collector waveguides is buttcoupled to the $9-\mu \mathrm{m}$-core fibers of a fiber array and the output from each fiber is measured independently by a photomultiplier (Hamamatsu H7732). During our characterization experiments the fiber array is used for convenience; in practical applications a photodetector array can be directly coupled to the chip.

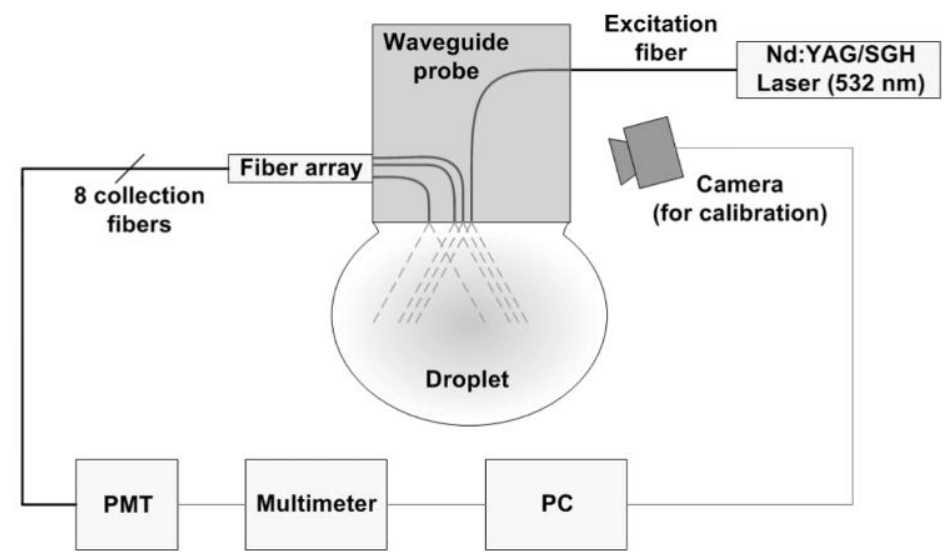

Fig. 3.17 - Measurement setup for the detection of light backscattered from a latex sphere suspension $(\mathrm{PMT}=$ photomultiplier; $\mathrm{PC}=$ computer $)$ 
Fiber-array-to-chip coupling is affected by misalignment, which causes nonuniform percentages of light intensity coupled from each collector waveguide to its corresponding fiber. In addition, the diced chip facet has a considerable roughness, which may be reduced when brought in contact with the sample droplet or when index-matching liquid is used. Hence, for this quantitative investigation the set-up is calibrated by reversely sending light through the array fibers and monitoring the intensity at the chip-droplet interface by a camera positioned above the droplet. The intensity is the same for all collector waveguides in case of equal fiber-waveguide coupling and a homogeneously diced chip facet.

To test the reproducibility of the results we perform two measurements, in between which the device is removed from the holder, cleaned and realigned with the fiber array. The collected number of photons as a function of lateral distance $d$ from the excitation point is shown in Fig. 3.15. As $d$ increases, we observe a pronounced decay in the detected signal. The output power measured from the first collector waveguide $(d=11 \mu \mathrm{m})$ is $5.6 \times 10^{-6}$ times the incident power. This means that an excitation power of $20 \mathrm{~mW}$ would generate a signal of $\sim 100 \mathrm{nW}$ that can be detected without the need for an ultrasensitive photomultiplier. As can be seen, the simulated curve agrees nicely with the experiment and thus validates the MonteCarlo model.

\subsection{Summary}

We have developed a semi-analytical model for describing waveguide probes, which is valid for single-mode as well as multi-mode waveguides with arbitrary crosssections. We have shown that for waveguide probes it is necessary to separately consider the NAs of the excitation and collector waveguides, despite the fact that the waveguides may have the same cross-sections. With the help of our model we have demonstrated that, in case of thin samples, integrated probes present higher efficiencies and resolution compared to large-core multi-mode fiber probes. Besides, their performance exceeds that of small-core multi-mode and single-mode fiber probes for all sample thicknesses. Moreover, by variation of the width, height and refractive index of the waveguide core we have shown that the collection efficiency of a waveguide probe increases in general with the number of guided modes, but the degree of increment strongly depends on the confinement factor of the highest-order mode. The model has been validated by our experimental results.

An integrated multi-waveguide probe was designed and tested for the delivery of excitation light and efficient, laterally resolved collection of light backscattered from highly scattering media. Our experimental results, which are confirmed by MonteCarlo simulations, demonstrate the feasibility of using integrated probes for backscattered light detection. The simulations also confirm that high depth resolution $(\sim 15 \mu \mathrm{m})$ is achievable with this type of probes. 


\section{Chapter 4}

\section{Confocal integrated light delivery and collection}

\subsection{Introduction}

$\mathrm{R}$

aman scattering produces extremely weak signals which can be more than six orders of magnitude lower in intensity than the excitation beam; therefore, in order to detect these signals, highly efficient light collection systems are needed. Therefore it makes sense to investigate whether channel waveguides are the most efficient devices for collecting backscattered light using integrated optics.

Increasing the collection efficiency of a channel waveguide is equivalent to increasing its effective collection area which increases with increasing physical dimensions or refractive-index contrast of the channel, as shown in Chapter 3 . It is not always possible to increase the latter since it depends on the material system used to fabricate the channel, and most of the time it is also a fixed constraint of the design, as for example when certain values of channel birefringence are needed or when single modality (in the vertical direction) of the waveguide is required. Under these assumptions we can think of achieving a higher collection efficiency by increasing the waveguide width; taken to the limit would mean to collect the light using a slab waveguide. Now, this option may seem unreasonable since most, if not all integrated optical devices require the channels to be single-mode in both vertical and lateral directions. However, if we find a way to distribute the light collected by the planar waveguide into many single-mode channels and then recombine it into a single channel we would obtain a collection device which has a much higher efficiency than that of a single channel.

A device that enables the distribution of light propagating in a planar waveguide into multiple channel waveguides is the star-coupler (see Fig. 4.1). A detailed description of the device can be found in [Dra89]. Star couplers have applications in many fields and are mainly used in the design of arrayed waveguide gratings (AWGs) [Dra91]. The latter, as explained in the next Section, are integrated optical devices which have both imaging and light dispersion properties [Smi88, Smi96]; for this reason AWGs are extensively used in wavelength division multiplexing and recently started to be used in spectroscopy and spectral OCT. In view of realizing our goal, a compact Raman device that combines the functions of light focusing, collection, and wavelength selection, we can think of making use of AWGs in a nonconventional way in order to exploit their advantages for our purpose. The device that we propose makes use of two arrayed waveguide gratings in a confocal arrangement as shown in Fig. 4.2: a first AWG for focusing the excitation light into 
the sample, and a second one for confocal signal collection. The device will be described in detail in this Chapter.

In the next Section the working principle of the arrayed waveguide grating is explained; while in Section 4.3 we describe how this device can be used for light collection and what its efficiency is compared to a channel waveguide. The comparison is carried out using both simulations and measurements which present excellent agreement. In the final part of Section 4.3 we give a proof of principle of the confocal arrangement of two AWGs by showing the results of an experiment in which a multi-wavelength image of a sample is recorded using a zero-order AWG for excitation and a wavelength selective AWG for light collection. In Section 4.4 we discuss the limitations of the approach, possible improvements and a broad range of potential applications. We finish the Chapter with a summary of our results.

\subsection{Working principle of the arrayed waveguide grating}

The arrayed-waveguide grating (AWG) was first proposed by Smit [Smi88] and subsequently reported by Takahashi et al. [Tak90] and Dragone [Dra91]. Since then it has developed into one of the most important devices in integrated optics. Its imaging and dispersive properties make it an ideal device for wavelength separation in wavelength-division-multiplexing and spectroscopic applications. The working principle of the AWG is briefly described referring to Fig. 4.1 (a).

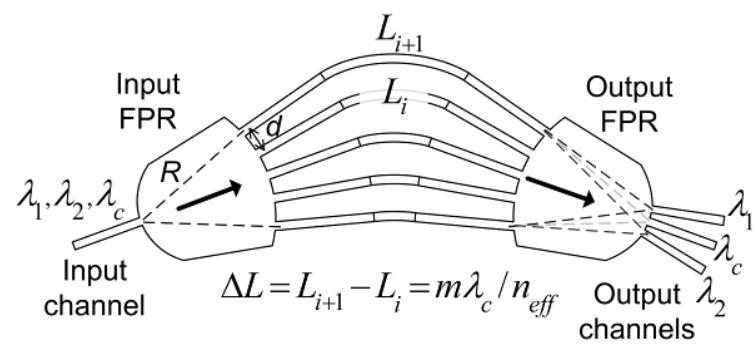

(a)

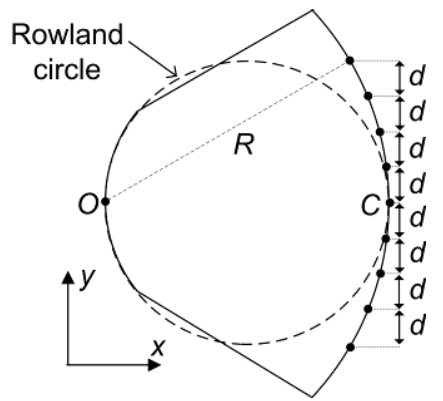

(b)

Fig. 4.1 - (a) Schematic layout of an AWG; (b) the schematic of a Rowland grating mounting where the dots indicate the positions of the arrayed waveguides arranged such that the chords have equal projections on the y axis.

Light from an input channel waveguide is guided to a free-propagation region (FPR) where it diffracts in the horizontal direction and is coupled to an array of channel waveguides which are arranged on a circle of radius $R$ (grating line) equal to the length of the FPR. On this circle the arrayed waveguides are spaced by a centerto-center distance $d \ll R$. Due to the limited number of arrayed waveguides, part of the light is lost to the sides of the array (spillover losses). This arrangement, comprising the input channel, the FPR, and the array of collecting waveguides forms a $1 \times N$ star coupler which couples the light from one input waveguide into $N$ arrayed waveguides. The arrayed waveguides have a linearly increasing length, and 
the length difference between adjacent waveguides is $\Delta L=m \lambda_{\mathrm{c}} / n_{\text {eff }}$, where $m$ is an integer, $\lambda_{\mathrm{c}}$ is the central wavelength of the AWG, and $n_{\text {eff }}$ is the effective refractive index of the arrayed waveguides at the central wavelength. Light exiting from the array enters a second FPR where the output terminations of the arrayed waveguides are again arranged on a circle with radius $R$ (as for the input FPR). The center of this circle coincides with the entrance facet of the central output channel of the AWG. With this arrangement, when light at wavelength $\lambda_{\mathrm{c}}$ is sent through the input channel, a circular wave front is generated at the output of the array, and the light is focused into the central output channel. For light at a different wavelength $\left(\lambda \neq \lambda_{\mathrm{c}}\right)$ the circular wave front generated at the output is tilted with respect to the one for $\lambda_{\mathrm{c}}$, and the focal spot is located at a different spatial position. Output channels can be placed at different positions at the output of the second FPR to collect individual spectral components of the input signal. The design just described makes use of a constant angular spacing between the arrayed waveguides. An alternative approach, which has the advantage of reduced aberrations, is the Rowland mounting [Hut82] where the arrayed waveguides, instead of being positioned at a constant angular spacing, are positioned such that chords (or center to center distances) have a constant projection on the $y$ axis, as shown in Fig. 4.1 (b). In this type of mounting the input and output channels are positioned on a circle (Rowland circle) and point towards the center $C$ of the grating line. The Rowland circle has a radius of $R / 2$ and is tangent to the grating line in $C$.

\subsection{An integrated approach to laser delivery and confocal signal detection}

As anticipated in the introduction to this Chapter, we propose a compact integrated optical device that combines the functions of light focusing, collection, and wavelength selection. The device makes use of two arrayed waveguide gratings (AWGs) in a confocal arrangement, displayed in Fig. 4.2. In its simplest design both AWGs have order $m=0$ such that they form two lenses, the first one focusing a single excitation beam into the focal spot at a desired depth inside the sample and the second one collecting the light backscattered from this focal spot into a central output channel. In this arrangement the device behaves like a confocal microscope in one lateral direction. 


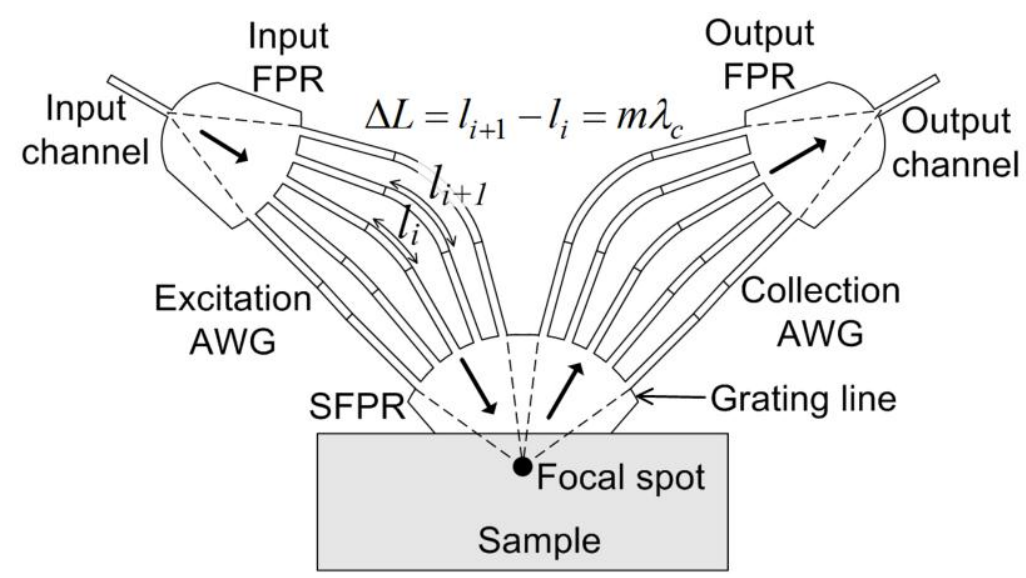

Fig. 4.2 - Schematic of the confocal arrangement of two AWGs (with central wavelength $\lambda_{\mathrm{c}}$ and order $\mathrm{m}$ ) which allows efficient illumination of, and signal collection from, a small focal volume below the sample surface.

Enhanced functionality can be achieved without increasing device complexity by use of a modified AWG design exploiting the phase relation between the individual channels, thereby adding wavelength selectivity. As a result, the focusing AWG can have more than one input channel, allowing one to focus several excitation wavelengths into the same focal spot. Likewise, the collecting AWG can resolve different wavelengths emitted from the focal spot, thereby enabling spectral analysis of the backscattered light, if designed with the required wavelength spacing and free spectral range (FSR). In the particular case in which all excitation and signal wavelengths fall into the FSR of a single AWG, the same AWG can be used for both excitation and collection.

The focusing and collecting AWGs are arranged in such a way that the output grating line - the interconnection line between the arrayed waveguides and the free propagation region (FPR) - of the focusing AWG lies on the same circle and, hence, is concentric with the input grating line of the collecting AWG (Fig. 4.2). In this way the output FPR of the focusing AWG and the input FPR of the collecting AWG merge together, forming a single sample-side FPR (SFPR). Ideally, its length is chosen such that, when in contact with a sample, the focal spot is located at the desired depth $d$ below the sample surface (for a given sample refractive index $n_{\mathrm{S}}$ ) in order to allow for maximum collection efficiency in the direction perpendicular to the device plane.

\subsubsection{Arrayed waveguide grating for the collection of backscattered light from a point source}

In our investigations, we focus on demonstrating that our approach improves the collection efficiency of signal light by more than an order of magnitude compared to standard light collection using a single-mode waveguide. Two similar AWGs are compared. The "reference" AWG is of a conventional design, having a single 64 
monomodal input channel for light collection and an AWG for spectral analysis with an input FPR of length $l_{\mathrm{FPR}}$, see Fig. 4.3(a). In contrast, the "collector" AWG is of the proposed design; see Fig. 4.3(b). Its input FPR is shortened to a length $l_{\mathrm{SFPR}}=$ $l_{\mathrm{FPR}}-d^{\prime}$, where $d^{\prime}$ depends on the distance $d$ of the input facet to the source (if $n_{\mathrm{S}}$ is equal to the effective index of the SFPR, then $d^{\prime}=d$ ).

For the experiments, a single AWG was fabricated in SiON technology with core and cladding refractive indices of 1.55 and 1.4485 , respectively, at $1.3 \mu \mathrm{m}$. The AWG had one input channel, an input FPR with $l_{\mathrm{FPR}}=5393.5 \mu \mathrm{m}, 400$ arrayed waveguides spaced by $8 \mu \mathrm{m}$ at the grating line, an output FPR, and 100 output channels with a wavelength spacing of $0.38 \mathrm{~nm}$ and a central wavelength of $1.3 \mu \mathrm{m}$. Each waveguide had a cross-section of $2 \mu \mathrm{m} \times 0.6 \mu \mathrm{m}$. The device was first used as the reference AWG, according to Fig. 4.3(a). Subsequently, its input free propagation region was repeatedly diced to be used as the collector AWG, according to Fig. 4.3(b), for measurements at different distances $d$.

As a source of signal light we used a diode laser (New Focus 6324) at a wavelength of $1284.6 \mathrm{~nm}$, which was coupled to either a standard $9-\mu \mathrm{m}$ fiber with angular aperture $\alpha=2 \arcsin \left(\mathrm{NA} / n_{\mathrm{d}}\right)=9.2^{\circ}$ or an ultra-high-numerical-aperture (UHNA) fiber with $\alpha=29^{\circ}$, with air (refractive index $n_{\mathrm{d}}=1$ ) between source and device. Subsequently, index-matching liquid with $n_{\mathrm{d}}=1.46$, close to the effective index of the waveguide, was placed between each fiber and the chip to reduce the angular aperture to $\alpha=5.7^{\circ}$ and $19.6^{\circ}$, respectively. The fiber output end was positioned at variable distance $d$ from the chip by a motion controller (Newport PM500) with nanometer precision. For accurate alignment of the collector AWG, the fiber end was placed in contact with the chip's facet and then moved along the $z$ axis to the collector's focal spot at distance $d$. The position was fine-tuned in the $x$ and $y$ directions by maximizing the signal from the AWG output channel centered at $1284.6 \mathrm{~nm}$. The output power was measured with a power sensor (Hewlett-Packard 8153A). 


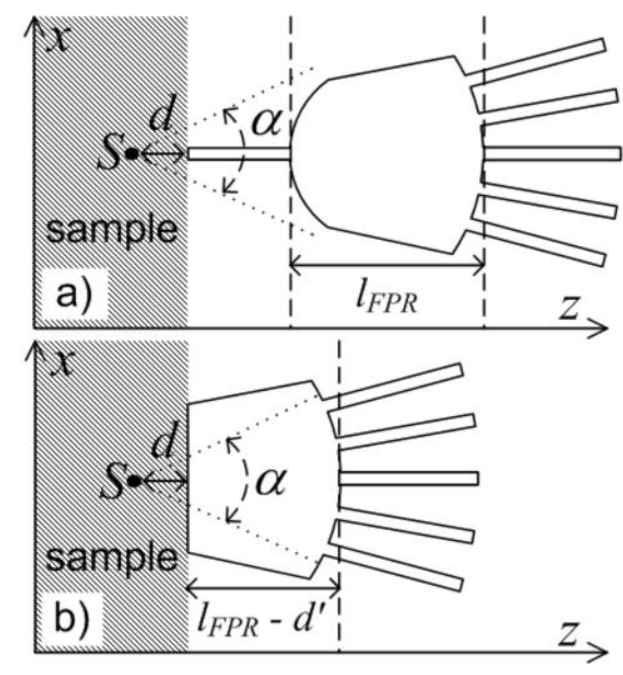

Fig. 4.3 - Input sections of (a) a "reference" (standard) AWG and (b) a "collector" AWG (the proposed device), both positioned at a distance $d$ from a source $S$.

Firstly, we performed measurements with the reference AWG for all four $\alpha=$ $5.7^{\circ}, 9.2^{\circ}, 19.6^{\circ}$, and $29^{\circ}$ at varying distance $d$. Secondly, measurements were performed on the repeatedly diced collector AWG at four distances $d=50 \mu \mathrm{m}, 100$ $\mu \mathrm{m}, 150 \mu \mathrm{m}$, and $200 \mu \mathrm{m}$. The collector AWG was measured only with indexmatching liquid $\left(\alpha=5.7^{\circ}\right.$ and $\left.19.6^{\circ}\right)$ to average out the roughness of the diced facet. The measured output power of reference and collector AWG is shown in Figs. 4.4(a) and 4.4(b), respectively, as a function of $d$. The sets of simulated and measured data are normalized independently from each other with respect to the power collected by the reference AWG at zero distance and $\alpha=19.6^{\circ}$, whose absolute efficiency under these conditions was measured to be $4 \%$. Furthermore, the collection efficiency of both devices was calculated as a function of $d$ by use of an analytical model based on Gaussian beam optics [Hal79]. All simulation parameters correspond to those used in the experimental setup.

Experiments and calculations show that at $d=0$, the curves for $\alpha=5.7^{\circ}$ and $9.2^{\circ}$ have a lower value than those for $\alpha=19.6^{\circ}$ and $29^{\circ}$ because of the better overlap between the waveguide mode (diameters $w_{\mathrm{x}}=2.58 \mu \mathrm{m}, w_{\mathrm{y}}=1.94 \mu \mathrm{m}$ ) and the mode of the UHNA compared to the 9- $\mu \mathrm{m}$ fiber (mode-field diameters of $3.3 \mu \mathrm{m}$ and 10.2 $\mu \mathrm{m}$, respectively). As $d$ increases, all curves exhibit a monotonous decay which is steeper for the reference compared to the collector AWG, because light collection by the former is limited in both $x$ - and $y$-directions by the cross-section of the input channel, while for the latter this limitation occurs only in the $y$-direction. This difference results in higher collection efficiency of the collector AWG compared to the reference AWG. As displayed in Fig. 4.4(c), the ratio of the power captured by the collector and reference AWG, i.e., the improvement in collection efficiency 
obtained by the proposed device, increases with source distance $d$ and angular aperture $\alpha$, to reach a value of approximately 15 at $d=225 \mu \mathrm{m}$ and $\alpha=29^{\circ}$.
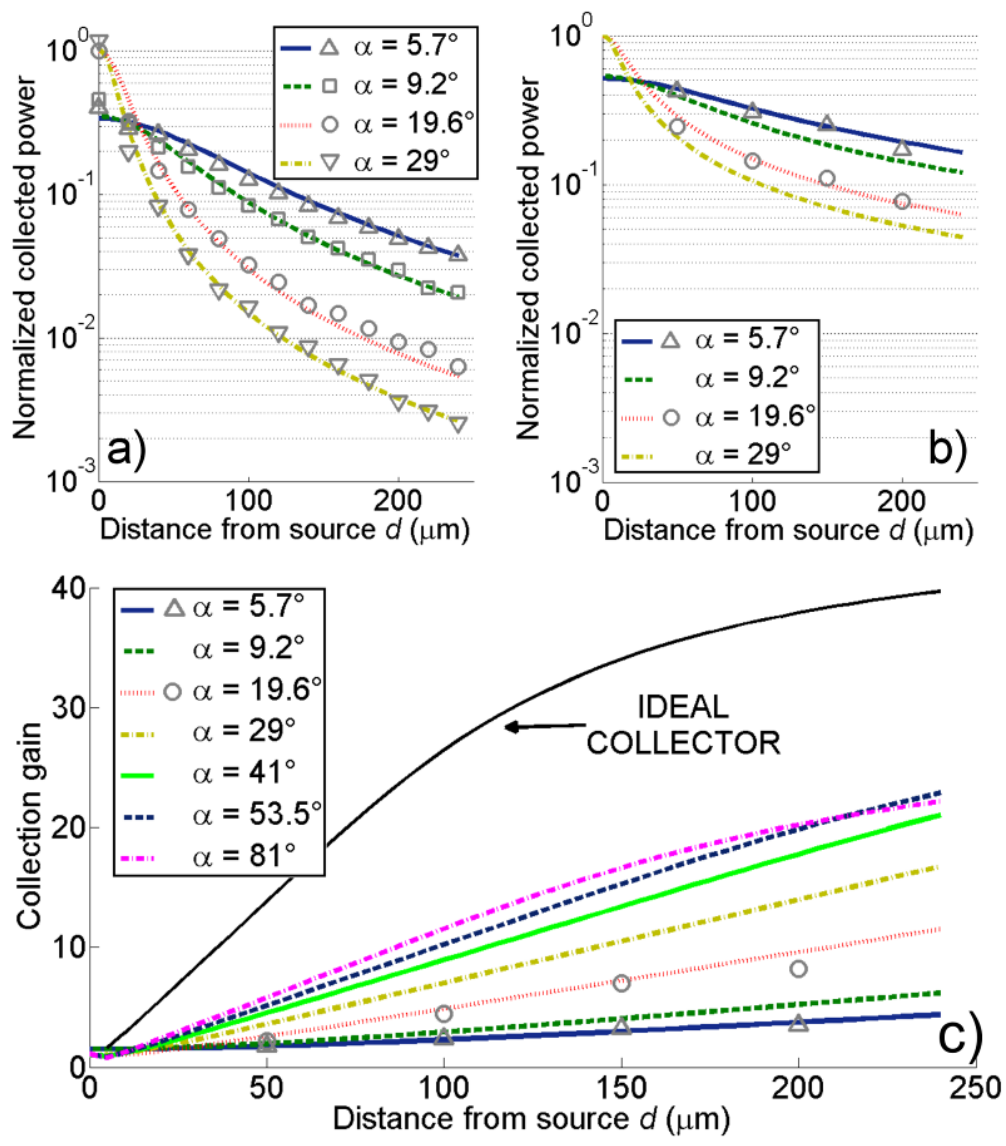

Fig. 4.4 - Comparison of measured (symbols) and calculated (lines) power collected by (a) the reference AWG and (b) the collector AWG, normalized to the power collected by the reference AWG at $d=0$ and $\alpha=19.6^{\circ}$, versus source-to-input facet distance $d$ for different values of the source angular aperture $\alpha$; (c) ratio of the power captured by collector versus reference AWG. This ratio is also shown for an ideal device with an infinitely extended SFPR.

In the investigated device the arrayed waveguides subtend an angle of $30.7^{\circ}$ from the focal point. For the value of $\alpha=19.6^{\circ}$, which is significantly smaller, the AWG spillover losses, which occur because part of the beam diverging from the source in the $x$-direction falls outside the area covered by the finite number of arrayed waveguides, are only $0.05 \%$. We calculated the collection efficiency also for higher values of $\alpha$, approaching the situation in which light inside a sample is scattered in all directions, thus representing a point source. The collection efficiency ratio improves with increasing $\alpha$ until $\sim 22$, however it levels off for $\alpha \geq 53.4^{\circ}$ and starts to drop for $\alpha \geq 66.7^{\circ}$. This behavior is due to an increase in the spillover losses 
as the emission angle of the source becomes larger. To overcome this problem the number of arrayed waveguides would have to be increased accordingly. For an ideal situation in which light is emitted over the whole solid angle and the number of arrayed waveguides is sufficiently large to capture all the light coupled into the SFPR, the collection efficiency ratio, displayed as the "ideal collector" line in Fig. 4.4(c), reaches a value of 26 already at a distance $d=100 \mu \mathrm{m}$ and further increases to 40 at $d=225 \mu \mathrm{m}$. This "ideal collector" would require an infinitely extended SFPR to collect all the light emitted by the source. It represents an upper limit to the improvement in collection efficiency when using waveguides with similar index contrast and layer thickness as those used in the examined case.

The spatial resolution of the focal spot provided by the collector AWG was determined by moving the fiber from the focal spot in all three directions. By taking the points at which the power decays by $3 \mathrm{~dB}$ (Fig. 4.5) we derive a volume $\Delta x \Delta y \Delta z$ of $4.4 \mu \mathrm{m} \times 56 \mu \mathrm{m} \times 65.5 \mu \mathrm{m}$ if the focal spot is located at a distance $d=224 \mu \mathrm{m}$ from the FPR input facet. This volume was measured using a $9-\mu \mathrm{m}$ fiber, the small angular aperture of which limits the collection volume in the vertical direction. In the more likely case of an isotropic emitter the vertical extension (which we estimate to be $144 \mu \mathrm{m}$ ) would be limited by the angular aperture of the SFPR.

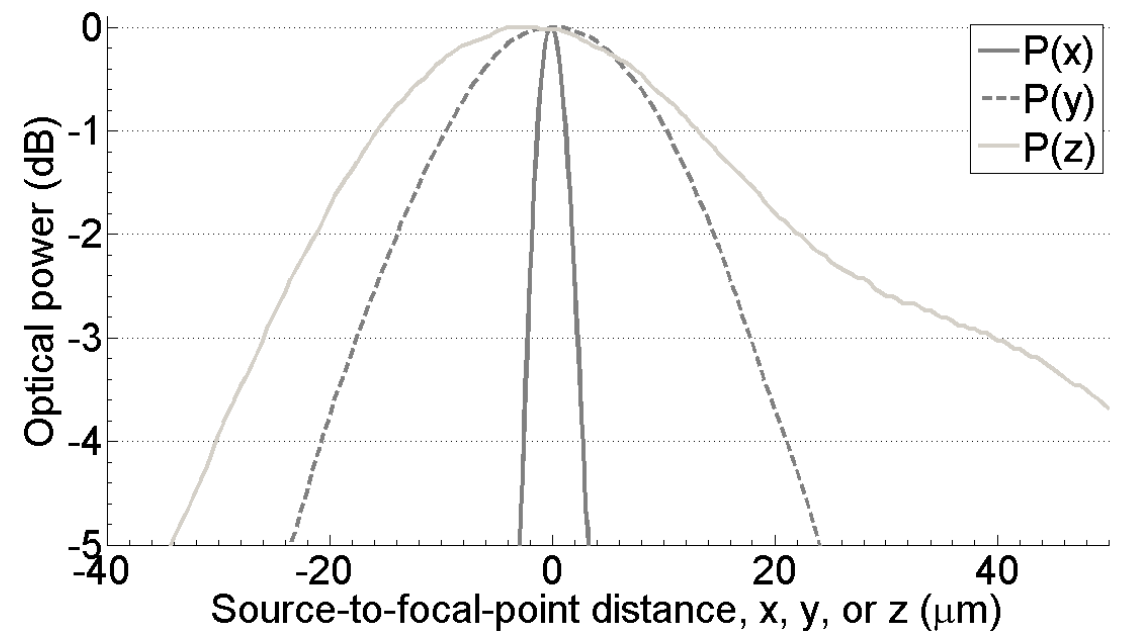

Fig. 4.5 - Measurement of the spatial resolution of the collector AWG for $d=224 \mu \mathrm{m}$. The output power of the AWG is plotted versus distance of the source from the focal spot in the three directions, $\mathrm{x}, \mathrm{y}$, and $\mathrm{z}$.

\subsubsection{Arrayed waveguide gratings for laser excitation and confocal signal collection}

In the previous Section we discussed our results on the efficiency of a single AWG used to collect the light scattered from a point through its free propagation region. Here we consider both, excitation and collection arrayed waveguide gratings in confocal arrangement. To demonstrate the proof of principle and show that the 
confocal arrangement works as expected we performed a simple experiment. A multi-wavelength image was recorded utilizing a device consisting of an excitation AWG of order zero in confocal arrangement with a collector AWG having 11 output channels spaced by $5 \mathrm{~nm}$ and centered at $831 \mathrm{~nm}$. As a sample we used the facet of a $9-\mu \mathrm{m}$-fiber array with $250-\mu \mathrm{m}$ spacing, partially coated with silver paint (Fig. 4.6 (a)). Two lasers operating at $831.4 \mathrm{~nm}$ and $838 \mathrm{~nm}$ were coupled into adjacent fibers to mimic multi-wavelength emission from the sample. For testing the confocal arrangement additional light from a super-continuum source was passed through a RG850 filter, focused through the excitation AWG, and reflected by the silver paint. The sample was positioned at the focal spot and scanned over an area of $620 \mu \mathrm{m} \times$ $60 \mu \mathrm{m}$ with $1 \mu \mathrm{m}$ step size. By detecting the signals from three output channels of the collector AWG centered at $831 \mathrm{~nm}, 841 \mathrm{~nm}$, and $856 \mathrm{~nm}$, respectively, three images were formed (Figs. 4.6 (b-d)). These images were then combined into a single image presenting both spatial and spectral information (Fig. 4.6 (e)).

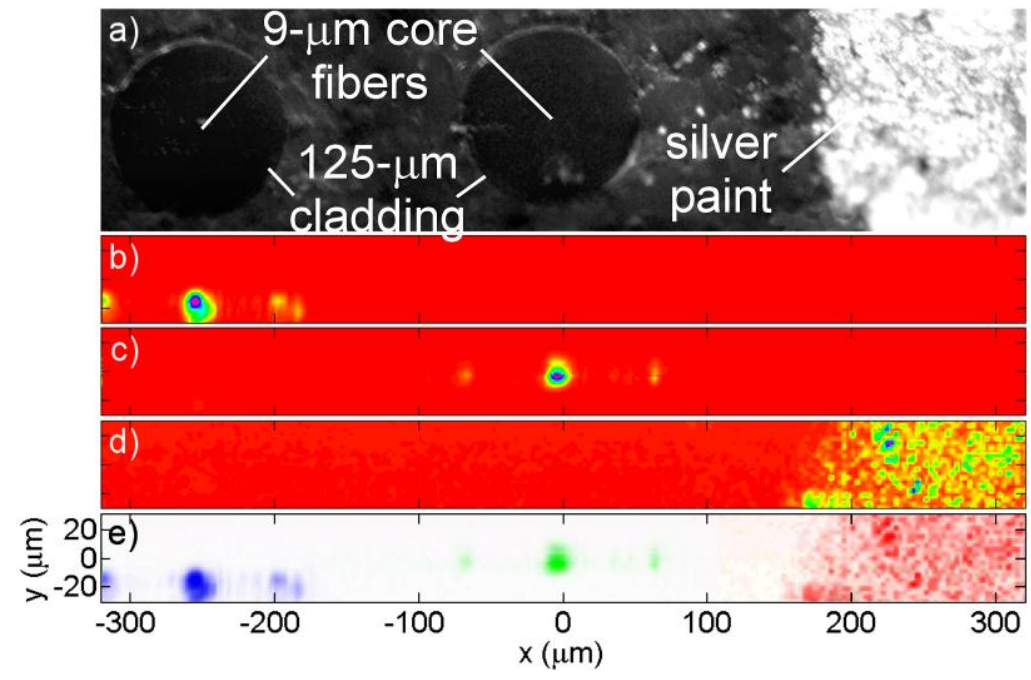

Fig. 4.6 - (a) 9- $\mu \mathrm{m}$ fiber array used as a sample; (b)-(d) measurement from output channels centered at $831 \mathrm{~nm}, 841 \mathrm{~nm}$, and $856 \mathrm{~nm}$; (e) resulting multi-wavelength image.

As can be seen from Figs. 4.6(b) and 4.6(c) two ghost images appear on both sides, at a distance of approximately $50 \mu \mathrm{m}$ from the fiber cores. The ghost images are due to the non-zero order of the collection AWG. To overcome this problem two zero-order AWGs must be used to focus and collect the light from the sample, directing the collected light into a third AWG to perform wavelength selection. In addition, it must be noted that the measurements in Figs 4.6 (b-c) are not making use of the confocal arrangement, since the light source in both cases is the fiber, while in Fig. 4.6 (d) the confocal arrangement is exploited. 


\subsection{Potential of the approach}

The confocal arrangement of two arrayed waveguide gratings is a novel method used for the first time in our work. Its potential has not yet been fully exploited and in the near future, may lead to new devices in the fields of microscopy and spectroscopy. In the following subsections we discuss some of the main properties of this novel device and, in particular, features which may be useful when targeting applications in the two afore-mentioned fields. Being a planar device, the most obvious limitations are the lack of focusing and limited collection of light in the vertical direction (orthogonal to the plane of the chip). In the last part of this Section we will elaborate on possible methods and techniques to improve the performance in the vertical direction.

\subsubsection{Imaging properties of the confocal AWG system}

In the following we will indicate with $\mathrm{z}$ the direction of propagation of light as it exits the device through the SFPR; we indicate with $x, z$ the plane of the chip and with $y$ the direction orthogonal to this plane. We will, therefore, sometimes refer to the directions $y$ as the vertical direction, and similarly $x$ as the horizontal direction. A schematic of the confocal AWG system and the reference coordinate system is shown in Fig. 4.7.

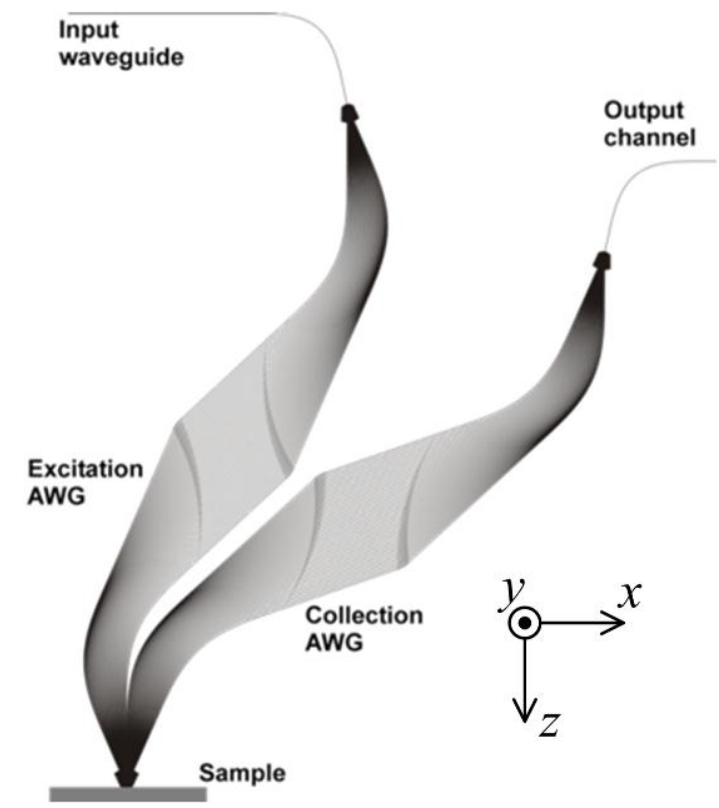

Fig. 4.7 - Schematic showing the confocal AWG system in contact with a sample.

When the confocal AWG system is used as an imaging device, an important parameter to be considered is the maximum resolution along $x$ and $y$ directions. In 
fact, since the device is a planar device and does not focus in the vertical direction it will necessarily have poor performance along this direction, while in the horizontal direction its performance will be higher. In order to quantify the performance of the device in terms of image quality we have performed some test measurements to obtain images of basic structures such as horizontal and vertical steps. From the measurements we have estimated the line spread functions (LSF) and modulation transfer functions (MTF) in the two directions.

The LSF is a measure of the ability of a system to form a sharp image of a line. An ideal imaging system would always image an infinitesimally thin line into an infinitesimally thin line. Of course this is not true for a real system with a limited resolution. The LSF can be calculated by differentiating the edge spread function (ESF) which is the response of the system to a step function. Mathematically the ESF is defined as [Hol08]:

$$
E S F=\frac{X-\mu}{\sigma},
$$

where $X$ is a vector containing the intensity values corresponding to the image of a step, $\mu$ is the average value of the intensity data $X$, while $\sigma$ is the standard deviation of the intensity data. By differentiating the edge spread function we obtain the line spread function:

$$
L S F=\frac{d}{d x} \operatorname{ESF}(x) .
$$

The modulation transfer function, defined as the modulus of the Fourier transform of the LSF [Ca197], gives us a measure of the faithfulness with which the details of an object are reproduced in the image. In particular, it is an estimate of how the intensity modulation (or contrast) of the object is transferred to the image by the imaging system.

The test measurements were performed by coupling light from a supercontinuum broad-band source (Fianium) into the excitation AWG through a single-mode fiber (P3-830A-FC-2). The AWGs were fabricated with SiON technology, having a waveguide cross-section of $2 \mu \mathrm{m} \times 0.52 \mu \mathrm{m}$ and core and cladding indices of 1.509 and 1.455 at $830 \mathrm{~nm}$, respectively. Both the excitation and collector AWGs were of zero order. The output of the collector AWG was coupled to a standard $9 \mu \mathrm{m}$ fiber and the intensity measured with a photodetector (HP81536A). The object, positioned at the focal plane of the AWG system, was scanned in the $x$ and $y$ directions with a step size of $0.1 \mu \mathrm{m}$ using a PM500 nanometer-precision stage. Between the object and the chip we placed a droplet of index matching oil with index of 1.47. The object consisted of a glass plate with a chromium rectangular feature deposited on the surface. When scanning through an 
edge of the rectangle, the high reflectivity contrast between the glass and the chromium resembles an ideal step function. The focal spot of the AWG system was found measuring its step response for different values of the distance $z$ between the object and the chip (see Fig. 4.8). The position of the focal spot was then obtained choosing the coordinate $z$ at which the highest contrast between chromium and glass was observed (equivalent to the steepest ESF), as shown in Fig. 4.9.

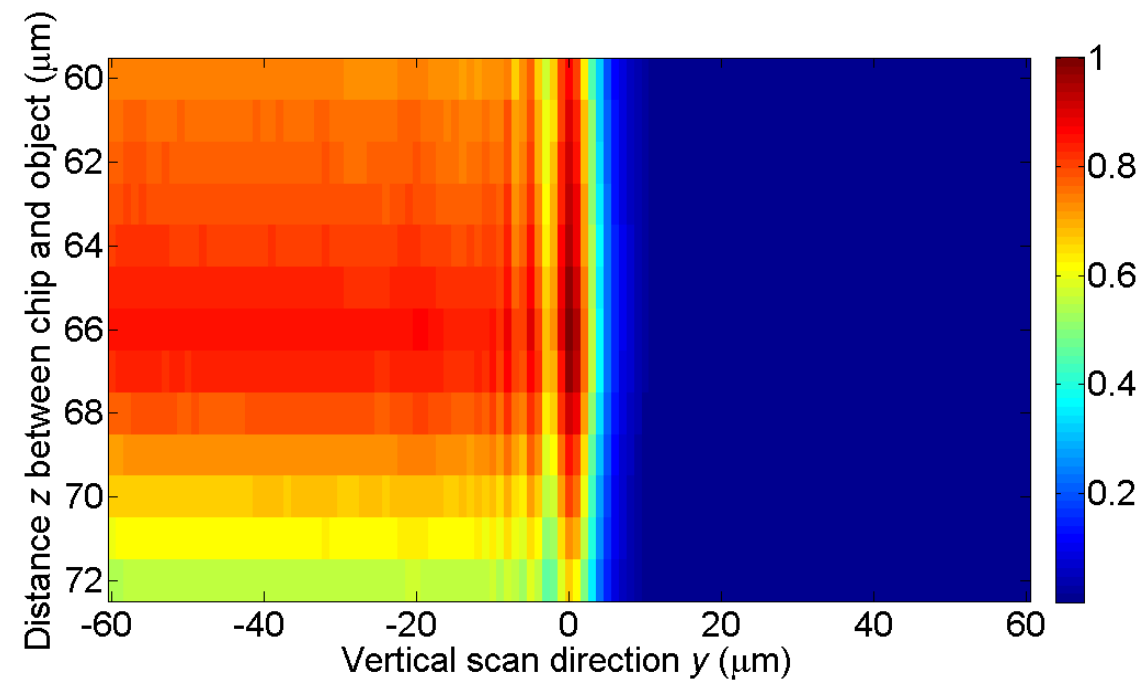

Fig. 4.8 - Set of scans of a reflection step (along $y$ ) at increasing distance $z$ between object and chip used to determine the exact location of the focal spot based on the ESF. The color scale goes from 0 (dark blue $=$ no reflection $)$ to 1 (dark red $=$ maximum reflection $)$ and is normalized to the maximum intensity of the back-reflected light.

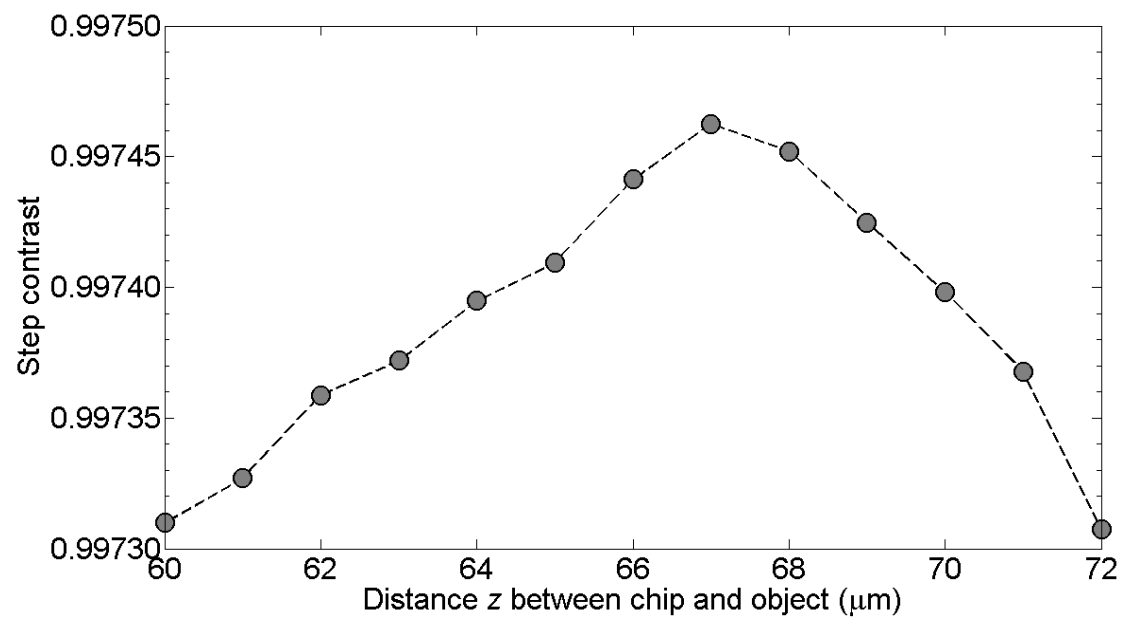

Fig. 4.9 - Contrast between the chromium and glass observed in the step response as a function of the distance $z$ between the object and the chip. The focal spot of the measured device is located at $67 \mu \mathrm{m}$, where highest contrast is observed. 
After the focal plane had been identified, the object was scanned first in the $x$ direction, then in the $y$ direction with a scan resolution of $0.5 \mu \mathrm{m}$. The scans are shown in Figs. 4.10 (a-b). We observe that in both cases the step response, for the glass-chromium interface, is not a monotonic function, since close to the interface some oscillations (or ringing artifacts) are present. These oscillations are usually found when coherent (as in our case) or partially coherent imaging is performed [Ind83]. We observe that in case of the $x$ scan, the overshoot is not so pronounced as in the case of the $y$ scan.
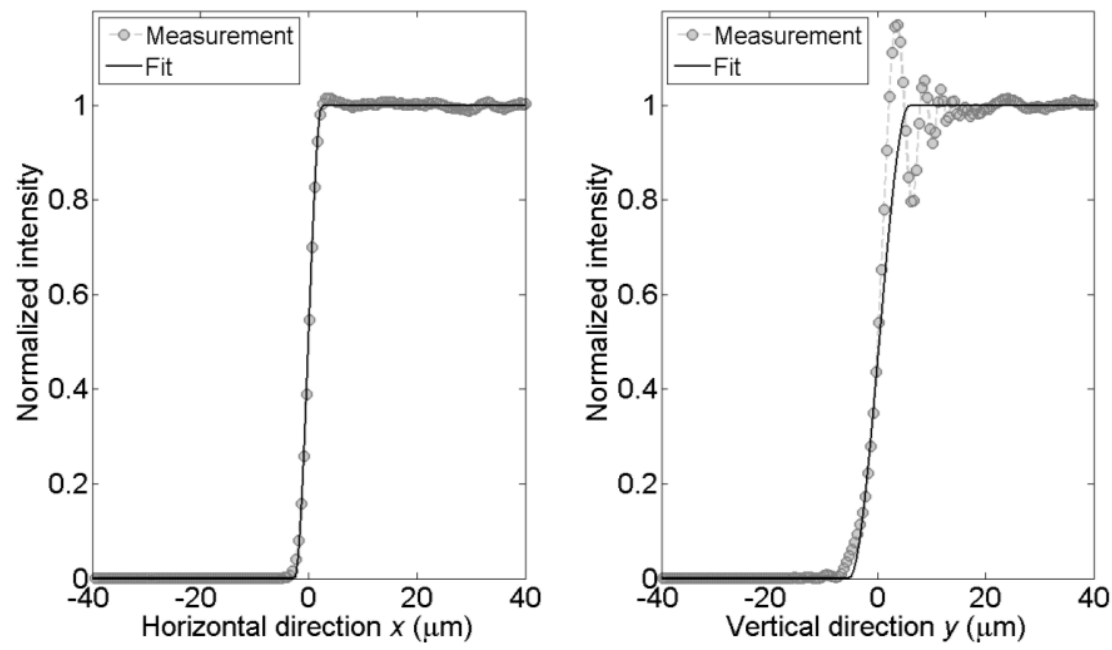

Fig. 4.10 - (a) Horizontal step response of the confocal AWG system; (b) Vertical step response of the confocal AWG system.

In order to use (4.2) to correctly estimate the LSF, the ESF must be a monotonic function. Otherwise the LSF (which is the derivative of ESF) will present negative values and loose its symmetry, which is a fundamental property that the LSF must have [Man97]. For this reason, in order to estimate the LSF and subsequently the MTF, we approximate the step responses by fitting the data points to a monotonic sigmoid function as described in [Ca197, Cal98], in the case for degraded edge responses. Different sigmoid functions are described in the literature, and we found that the flexible sigmoid function described in $\left[\mathrm{Yin}_{0} 3_{\mathrm{a}}, \mathrm{Yin}_{\mathrm{b}} \mathrm{b}\right.$ ] fits best to our data. This function has the form:

$$
f(x)=(A-B)\left(1+\frac{x_{2}-x}{x_{2}-x_{m}}\right)\left(\frac{x-x_{1}}{x_{2}-x_{1}}\right)^{\frac{x_{2}-x_{1}}{x_{2}-x_{m}}}+B, \quad \text { where } x_{1} \leq x_{m}<x_{2} .
$$

In (4.3) $A$ is the highest value reached by the step, and $B$ is the lowest value; while the parameters $x_{1}$ and $x_{2}$ are the points where the sigmoid starts and ends, respectively. In other words, for values of the coordinate $x$ smaller than $x_{1}$ the function $f(x)$ has a constant value of $B$, while for values of $x$ greater than $x_{2}, f(x)$ has 
a constant value of $A ; x_{\mathrm{m}}$ is an intermediate coordinate where $f(x)$ has the value of ( $A$ $+B) / 2$. The results of the fit are shown in Figs. $4.10(\mathrm{a}-\mathrm{b})$ with the solid line. From these curves the LSF for the horizontal and vertical direction can be easily calculated using the expressions (4.1) and (4.2). The line spread functions are shown in Fig. 4.11. We notice that the FWHM of the horizontal LSF is $\sim 3.5 \mu \mathrm{m}$, while the FWHM of the vertical LSF is $\sim 7.9 \mu \mathrm{m}$.

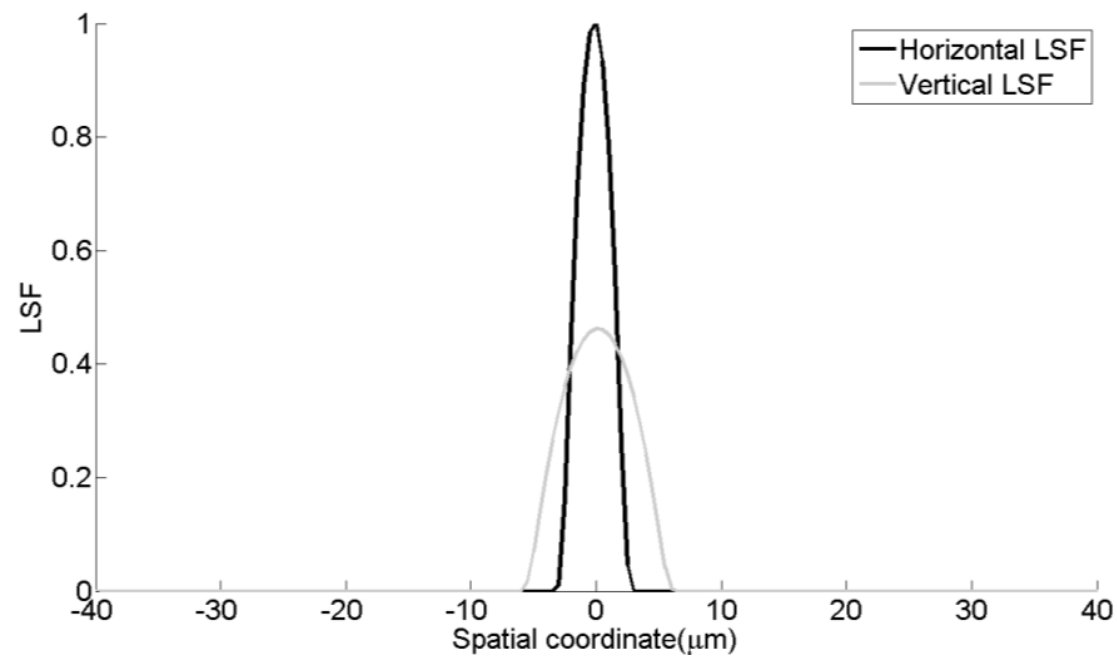

Fig. 4.11 - Calculated line spread functions of the confocal AWG system in the $x$ and $y$ directions.

By taking the modulus of the Fourier transform we obtain the modulation transfer functions (MTF) for both, $x$ and $y$ directions, which are shown in Fig. 4.12.

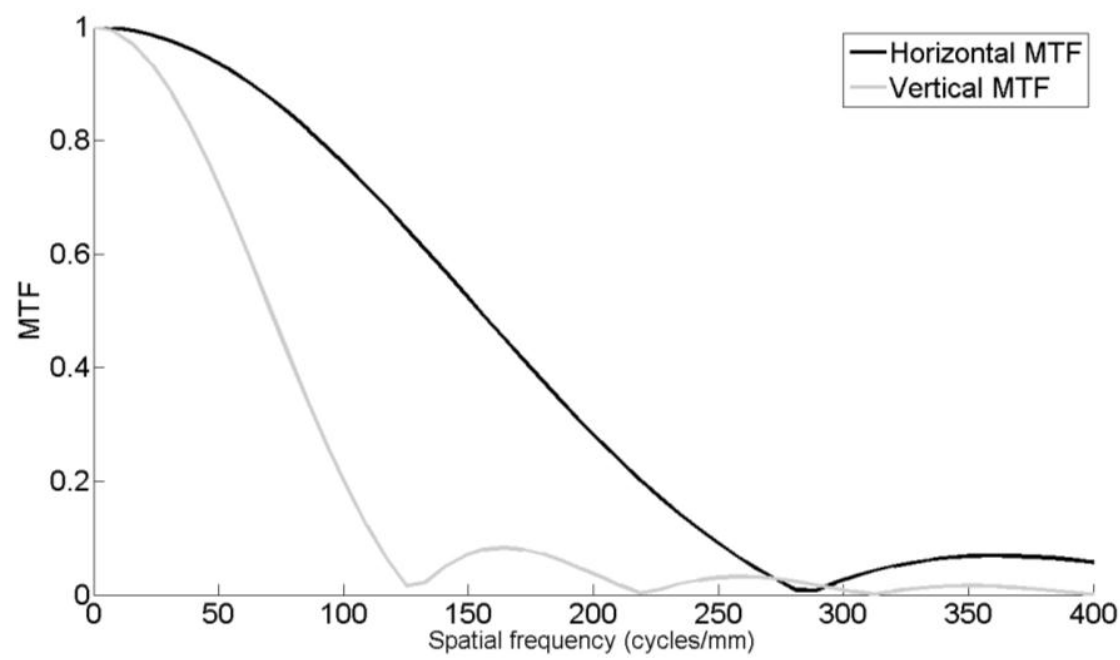

Fig. 4.12 - Calculated modulation transfer functions of the confocal AWG system in the $x$ and $y$ directions. 
Conventionally the maximum resolvable spatial frequency is assumed as the value at which the MTF decays by $3 \mathrm{~dB}$ from its maximum. By using this definition we obtain that the maximum horizontal frequency that can be resolved by the confocal AWG device used in the experiment is 150 cycles $/ \mathrm{mm}$ (feature size of 3 $\mu \mathrm{m}$ ), while being only around 70 cycles $/ \mathrm{mm}$ (feature size of $7 \mu \mathrm{m}$ ) in the vertical direction.

\subsubsection{Focusing in the vertical direction}

Focusing in the vertical (or $y$ ) direction cannot be achieved with the current configuration of the device. It is, however, possible to improve the device performance in the vertical direction by using vertical tapering of the SFPR in order to expand the mode and reduce the diffraction angle of light (in the $y$ direction) exiting the waveguide. A more fundamental improvement can be achieved by placing a cylindrical lens in front of the SFPR as shown in Fig. 4.13, thus obtaining an actual focus in the y-direction as well. The lens could, in principle, be fabricated by depositing a channel structure of boron-phosphorus doped $\mathrm{SiON}$ and performing reflow at low temperatures $\left(800^{\circ} \mathrm{C}\right)$ [Wör08].

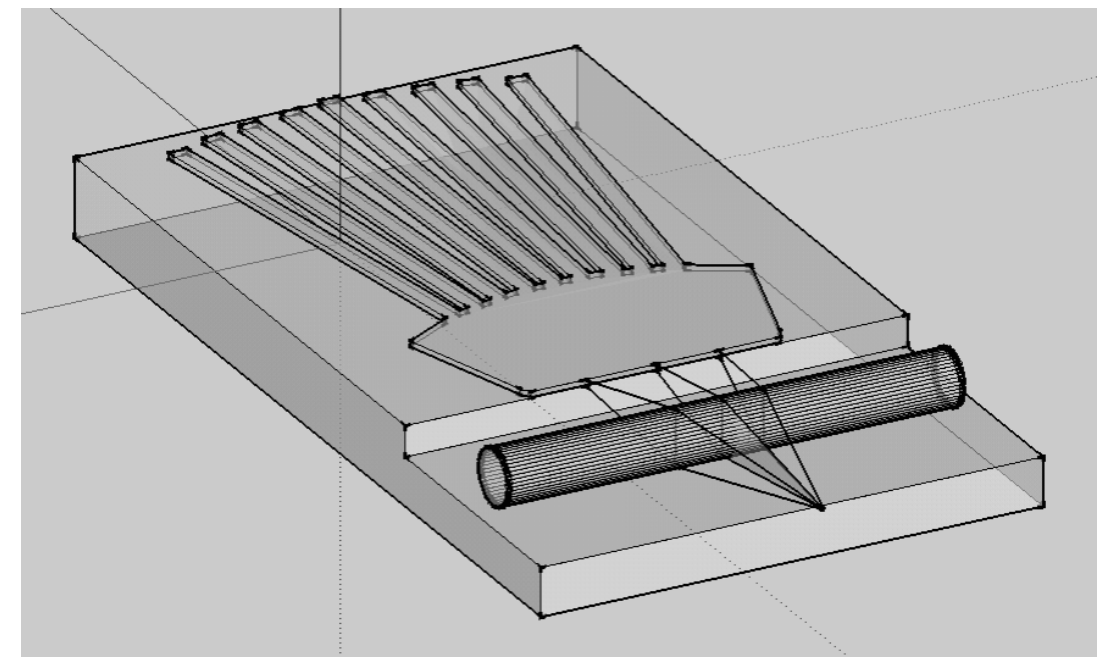

Fig. 4.13 - Schematic of a cylindrical lens positioned in front of the excitation AWG to achieve vertical focusing of the excitation wavelength.

\subsection{Summary}

In this Chapter we described a method to focus light on a sample and collect the backscattered light from the focal spot using optical devices integrated on a chip, without the need for external optical elements. In particular, the device used to focus the light and to collect the backscattered light is an AWG, in which the input FPR has been partially diced. We demonstrated that this method enables higher collection efficiency than that achieved using a channel waveguide. We also gave a proof of 
principle, showing that the device can be used as a microscope for imaging at multiple wavelengths.

The limitations and possible improvements of the approach have also been discussed in Section 4.4. 


\section{Chapter 5}

\section{Integrated Raman spectrometers}

\subsection{Introduction}

I

$\mathrm{n}$ this Chapter we focus on the design of integrated Raman spectrometers for the applications outlined in Chapter 1, which require detection of the Raman signals from skin and teeth. Since the two applications regarding signal detection from skin have very similar specifications, in this Chapter we only address the NMF application.

Among the requirements of a Raman spectrometer, besides those of efficient light collection and wavelength selectivity in the Raman signal range, is also a high rejection of light at the excitation wavelength. For design simplicity we separate these requirements into three or four different functional blocks, as shown in Fig. 5.1, implementing each block with a different optical circuit (or device). As will be discussed, only three functional blocks are sufficient for the skin applications (water concentration and NMF detection), whereas a fourth block (polarization splitter) is needed for the dental application which requires a separate analysis of the transverse-electric (TE) and transverse-magnetic (TM) polarization components of the Raman signal.

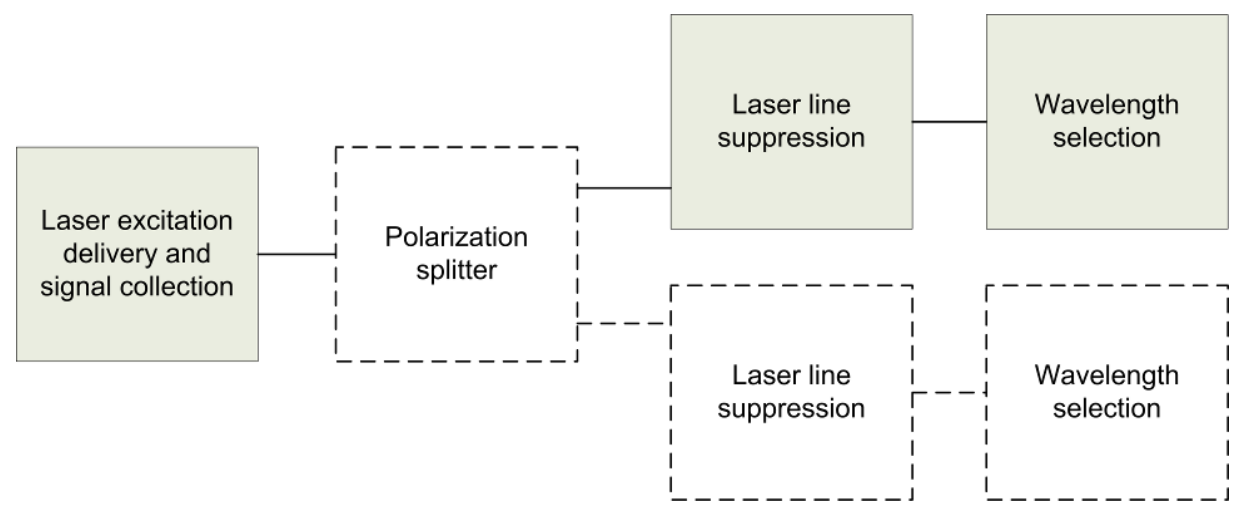

Fig. 5.1 - Functional block diagram of Raman spectrometer. The dashed blocks are optional and depend on the application.

We start Section 5.2 with a motivation of the choice to use arrayed waveguide gratings (AWGs) as the wavelength selection devices in all the Raman Pen applications. We then present, in the same Section, a novel AWG layout on which we base the wavelength selection devices for the skin application. The novel layout makes use of identical bends in all the arrayed waveguides to avoid systematic phase errors arising from the use of different bends in the conventional layouts. As will be described, the identical bend layout can be used to design AWGs with any 
diffraction order. A more detailed description of this layout is given in the Appendix A. In Section 5.3 we present the Raman spectrometer for the skin application, starting with a block diagram of the integrated spectrometer. As the first block, laser delivery and signal collection, has already been described in the previous Chapter, the detailed description in this Chapter will be limited to the wavelength selective device. As mentioned above, the wavelength selective device is a broad-band AWG, based on the identical-bend layout. We present experimental results on the characterization of the fabricated device. We also present, in the Appendix B, the design details and preliminary measurements of the laser suppression filter, which is based on cascaded Mach-Zehnder interferometers (MZIs). In Section 5.4 we discuss the design of the spectrometer for the dental application. As before, the wavelength selective device is based on the AWG. We compare the simulation and experimental results for this device. The design of the polarization splitter for the dental application is addressed in the Appendix C, while for the laser suppression filter a similar layout as discussed in the Appendix B can be used.

\subsection{The arrayed waveguide grating for wavelength selection in Raman spectroscopy}

The aim of the Raman Pen project is to realize a hand-held device, which is emphasized by the word "pen" in the title. This does not only translate into the device being compact, but also fast. The speed of the device is a very important aspect in this case, since both the operator who is holding the device and the subject under examination will inevitably move during the measurement. For in-vivo measurements the device must be able to acquire a Raman signal over the entire spectral region of interest in a matter of a few seconds [Han00].

When performing spontaneous-Raman measurements, the typical integration time needed for the signal to be resolved from the noisy background ranges from tenths of a second to even tens of seconds, depending among other things on the intensity of the excitation laser and the efficiency of the collection optics. Now, if we imagine performing a Raman measurement scanning over a spectral range of 20 $\mathrm{nm}$, with a wavelength resolution of $0.2 \mathrm{~nm}$ (as required by the dental application), and using an integration time of $10 \mathrm{~s}$ for each of the 100 wavelength points, we would need $1000 \mathrm{~s}$ to complete the measurement. This time is unacceptable for invivo measurements. However, if we acquire all the wavelength points simultaneously, then only $10 \mathrm{~s}$ would be needed for the entire measurement. The difference between the two cases is that, in the first case, a tunable filter is needed with a single detector at its output, while in the second case we need a dispersive element that spatially distributes the spectral components of the input signal over 100 detectors. This second option is 100 times faster and is one of the reasons for choosing an AWG for wavelength selection in this project. The AWG, in fact, through its output terminals enables simultaneous access to all spectral components of the input signal, leading to a fast measurement irrespective of the required spectral range and resolution. It is also true that the AWG is not the only dispersive 
device that can be realized in integrated optics. For example we can achieve the same functionality using an Echelle grating, but this device has a complex fabrication process with very stringent tolerances on the side wall roughness of the grating walls and their verticality, besides involving more than one lithographic step [Jan04]. The fabrication process of AWGs, on the other hand, involves only a single lithographic step, which is tantamount to low cost, making them ideal for our application.

We already described the working principle of the AWG in Section 2 of Chapter 4. Here, we will focus on a novel geometrical layout for the AWG which presents improved performance when comparing its response to that of conventional layouts. A typical cause of degradation in the response of an AWG is the presence of phase errors across the arrayed waveguides; these can be regarded as deviations of the optical path lengths from the designed values and can be divided into two categories. The first category comprises phase errors that arise from the device fabrication process; these phase errors are caused by random variations in the waveguide width, which depend on the resolution of the mask used in the photolithographic process [Lee01], as well as waveguide side-wall roughness and non-uniformities of the guiding layer properties, such as its refractive index and thickness [Goh97]. The second category includes systematic phase errors due to the design of differently bent sections in the arrayed waveguides. In each bend light experiences an effective refractive index which is a function of the bending radius. Usually, the difference between the effective indices in the straight and bent sections of an arrayed waveguide is very small (between $1 \times 10^{-5}$ and $1 \times 10^{-4}$ ); however, when the arrayed waveguide bends over a large angle (i.e. $\sim \pi$ ) and has a small bending radius, the induced phase deviation from the designed value may become significant. In particular, in conventional AWG layouts, such as horseshoe and s-shaped AWGs [Ada93, Smi96] in which the bends in the arrayed waveguides all differ in radius and length, the changes in the optical path length are different from waveguide to waveguide within the array, leading to a distortion of the wave fronts in the second FPR with a consequent defocusing effect.

Systematic phase errors are predictable and can, therefore, be accounted for when designing the relative delays between adjacent arrayed waveguides. However, this requires numerical simulations and may not lead to perfect cancellation of these phase errors due to both, simulation inaccuracies and fabrication tolerances. The AWG introduced by Takahashi et al. [Tak90] consisted of identical bends (four $90^{\circ}$ bends in each arrayed waveguide), however the input and output sections of the arrayed waveguides were parallel to each other and not arranged on a star coupler. The use of star couplers in AWGs was first proposed by Dragone in 1991, in which two star couplers were interconnected by arrayed waveguides of unequal lengths and with a fixed angular separation [Dra91]. In the star-coupler configuration the nonzero angle between adjacent waveguides of the array makes it difficult to use identical bends in the AWG layout. A partial solution to the problem was proposed in [Soa08], where only bends with equal bending radius were used in the arrayed 
waveguides. This, however, did not completely cancel the systematic phase errors, since the lengths of the bends were not equal across the array.

\subsubsection{The identical bend design}

In this work we propose a new layout in which all the arrayed waveguides have identical bends. Our design is based on arranging the arrayed waveguides with a constant angular spacing, in this way deviating from the Rowland mounting although the input and output channels are still positioned on the Rowland circle. This deviation, in terms of position of the arrayed waveguides, is in general very small, as will be shown in Section 5.3.1. In our fabricated device the positions of the arrayed waveguides deviate from the Rowland mounting by $165 \mathrm{~nm}$ maximum, which is less than the resolution of the e-beam mask used in the fabrication. In Sections 5.2.2 and 5.2.3, we show that the identical bend layout can be used for both, low-order and high-order AWG designs; besides eliminating the systematic phase errors, in the case of high-order AWGs this layout presents the additional advantage of reduced area compared to the conventional horse-shoe layout. In Section 5.3.1 we present experimental results on the characterization of a broadband AWG which makes use of the novel design. As required for the skin applications, this AWG has a resolution of $5.5 \mathrm{~nm}$ and a polarization-independent response over a very large spectral range of $215 \mathrm{~nm}$ (the maximum observed shift between the responses for the two polarizations is a factor of 10 smaller than the resolution).

As a starting point we considered the broadband anti-symmetric AWG model introduced by Adar et al. [Ada93]. The geometry is shown in Fig. 5.2.

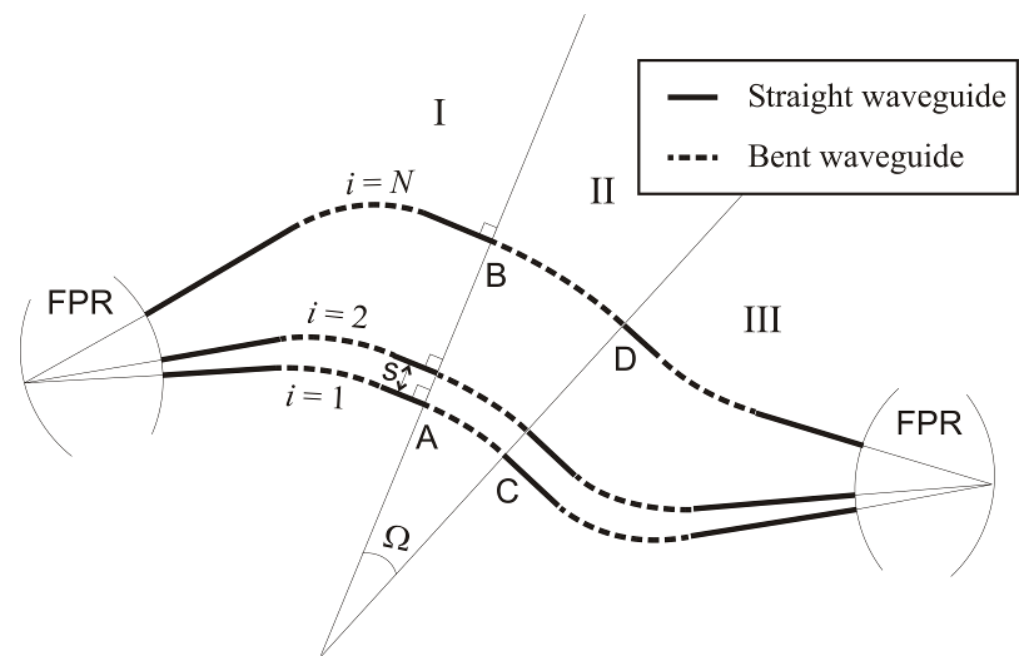

Fig. 5.2 - Anti-symmetric layout of a conventional broadband arrayed waveguide grating having $N$ arrayed waveguides. The free-propagation-regions (FPRs) are indicated schematically. 
In this model each waveguide is composed of four straight sections interconnected by three arcs. The layout can be divided into three parts; parts I and III are identical but rotated by an angle $\pi+\Omega$. In part I, each arrayed waveguide is bent to normal incidence on line $\overline{\mathrm{AB}}$. Two conditions are imposed on the model for the waveguides of part I: firstly, the separation $s$ between adjacent waveguides on line $\overline{\mathrm{AB}}$ is constant; secondly, the length of each waveguide is increased with respect to that of the previous waveguide by a constant amount. In this way, if part I is directly connected with part III, all waveguides will necessarily have the same length. The length difference between the arrayed waveguides is introduced by the arcs of the interposed part (part II). A more detailed description can be found in [Ada93].

Smaller size of the AWG can be achieved with smaller bending radii; yet, particular care must be taken to keep the waveguide bend losses at an acceptable level. Low-loss curved waveguides can be achieved by introducing non-linear curvatures [She08]. However, if all the arrayed waveguides exhibit different nonlinear bends, the phase errors introduced by small bending radii can reach significant levels. As will be explained in the next Section, in high-order conventional AWGs there is a trade-off between device size, losses, and systematic phase errors, which is a key motivation for developing a layout that intrinsically avoids the latter. Introducing the length differences only in the straight sections of the arrayed waveguides would allow one to design bends with any desired shape without introducing systematic phase errors to the AWG, because the same bend is used in all arrayed waveguides.

We have implemented an AWG layout in which all the waveguides make use of identical bends, while the length difference $\Delta L$ between adjacent waveguides is obtained in the straight waveguide sections. Our layout is inspired by Adar's work [Ada93], with the main difference that for low-order designs we do not use a central part to introduce the length differences (a central part will be reintroduced for highorder designs, as discussed later); instead, we only use two parts which are shown in Fig. 5.3. A prime is used to distinguish the quantities of the right half, see Fig. 5.3 (b), from those of the left half, see Fig. 5.3 (a). For both halves we impose that the separation $s$ between two adjacent waveguides on line $\overline{\mathrm{AB}}$ is constant. However, for the left half we impose that the length of each waveguide is increased with respect to the length of the previous waveguide by the amount $l_{i}-l_{i-1}=a+\Delta L / 2$, where $a$ is an arbitrary constant; in contrast, for the right half, we impose that $l_{i}^{\prime}-l_{i-1}^{\prime}=\Delta L / 2-a$. 

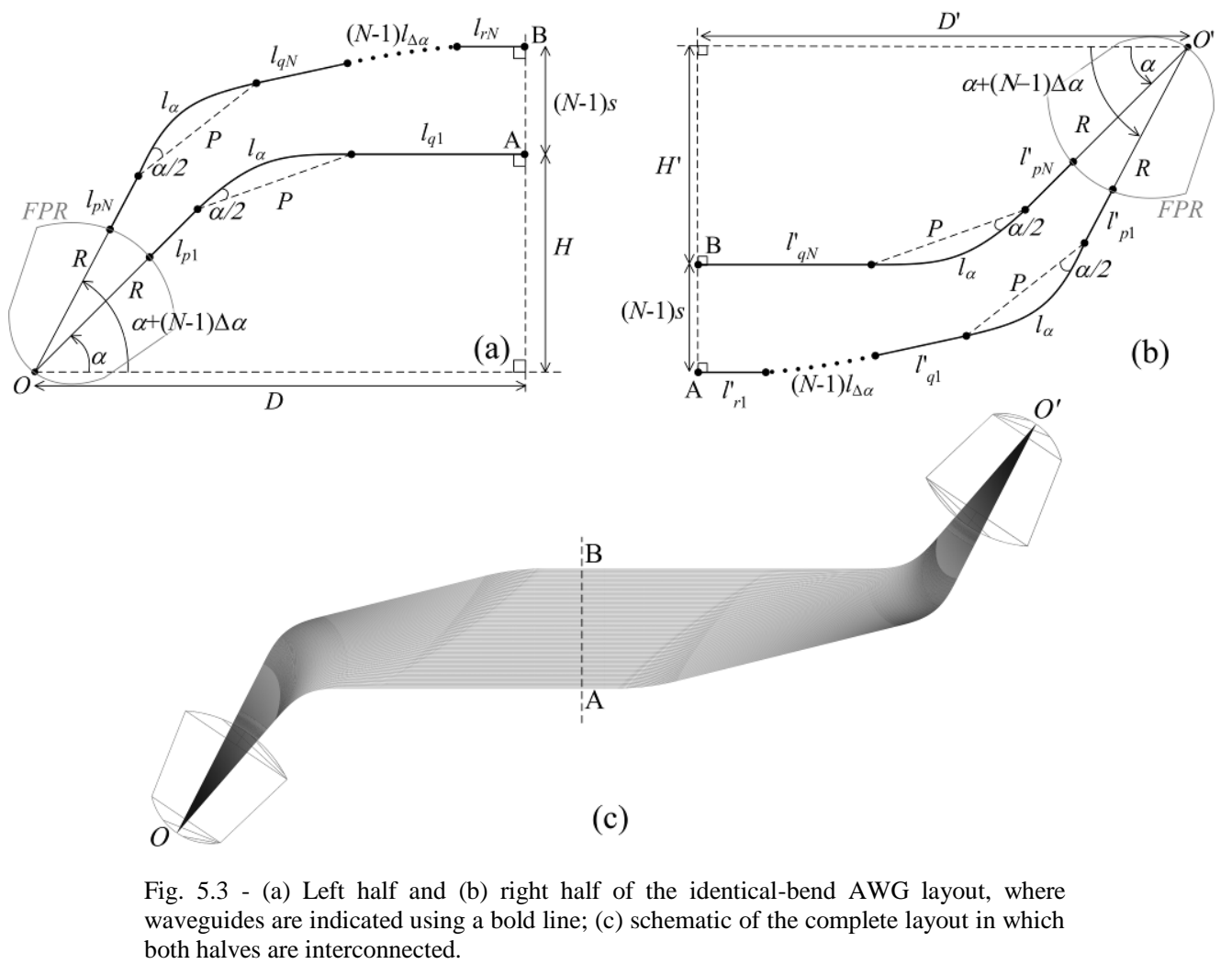

When both halves are connected to each other, the length difference between adjacent arrayed waveguides will be $\Delta L$. Our layout, for grating orders $m>0$, is not anti-symmetric, which is different from Adar's design.

In Fig. 5.3 (a) we present a schematic of the left half of the AWG. $R$ is the radius of the FPR and $N$ is the total number of arrayed waveguides. For clarity, only the first waveguide $(i=1)$, which we will refer to as the 'reference waveguide', and the last waveguide $(i=N)$ are shown. The angle between any two adjacent waveguides of the array is $\Delta \alpha$, while the angle that the reference waveguide makes with the horizontal axis is indicated by $\alpha$. The device makes use of only two types of bends: type 1 bends by an angle $\alpha$, while type 2 bends by $\Delta \alpha$. These bends are the building blocks to be used in equal numbers in all the arrayed waveguides. As shown in Fig. 5.3(a), all waveguides are bent to normal incidence on line $\overline{\mathrm{AB}}$. This means that the waveguide with $i=1$ is bent by an angle $\alpha$, the waveguide with $i=2$ by $\alpha+\Delta \alpha$, and so on. The $i^{\text {th }}$ waveguide, which is bent by $\alpha+(i-1) \Delta \alpha$, has one bend of type 1 and $i-1$ bends of type 2 .

Figure 5.3 (b) displays a schematic of the right half of the AWG. The waveguides are numbered in the same order as on the left half; the lowest waveguide 
is number 1 and will be connected to waveguide number 1 of the left half; the uppermost waveguide is number $N$ and will be connected to waveguide number $N$ of the left half. On the right half, waveguide $N$ includes one bend of type 1 , waveguide $N-1$ has one bend of type 1 and one bend of type 2 , and so on. When we connect all the waveguides of the left half with those of the right half, each arrayed waveguide $i$ has the same number of bends, namely two bends of type 1 and $N-1$ bends of type 2. Consequently, any bend type (e.g. circular bend, linear bend, nonlinear bend) and length can be chosen. Nevertheless, it is preferable to find a good compromise between the length of a bend and its bending radius to reduce propagation losses as well as the size of the device. For example, the bend of type 2, which is repeated $N-1$ times in each waveguide, has typically a very small length (on the order of 10-20 $\mu \mathrm{m}$ ), since the angle $\Delta \alpha$ is typically very small $(\Delta \alpha=d / R \sim$ $10^{-3} \mathrm{rad}$, see Fig. 4.1). For this reason, a small bending radius can be used if the number of waveguides $N$ is small, while a larger minimum bending radius is preferable in case of large $N$, i.e., when many bends of type 2 are connected to each other in sequence. For the bends of type 2, circular bends can avoid bend-to-bend transition losses and undesirable periodicity in each waveguide (as would be the case when connecting many nonlinear or linear bends to each other), whereas nonlinear bends can eliminate the straight-to-bend transition losses introduced by circular bends. Furthermore, in high-index-contrast waveguide systems, an offset is usually required when interconnecting straight and bent waveguides to reduce the mode mismatch at the junction. In this case the proposed AWG geometry gives more flexibility in the design without leading to a higher design complexity than when using the conventional geometry. For example, while the conventional Sshaped AWG has six straight-to-bend interfaces requiring the offset, the proposed AWG only requires four offsets, since the bends of type 1 can be non-linear bends with a gradual straight-to-bend transition. The details of the geometry are discussed in the Appendix A. In Fig. 5.3 (c) we present, for clarity, the complete layout of the AWG.

\subsubsection{Design and simulation of high-order AWGs with the identical-bend layout}

The proposed identical-bend layout can also be used to design high-order AWGs. Generally, with increasing order of the AWG the device becomes larger; for very high orders the phase errors, in particular those arising from fabrication nonuniformities, may become so significant (tens of radians) that a procedure of photosensitive phase compensation is necessary after device fabrication [Tak02]. To limit these phase errors the device size must be reduced; this is done by choosing a smaller value for the minimum bending radius, which, on the other hand, results in increased losses and increased systematic phase errors in a conventional AWG. In contrast, in the case of an identical-bend AWG it results only in an increase of the losses. 
As shown in Fig. 5.4, the design of a high-order AWG using the identical-bend layout is performed by simply interconnecting the two parts that have been described in the preceding Section through an intermediate part, in order to introduce the necessary length differences. The $i^{\text {th }}$ waveguide of the intermediate part, which is connected to the $i^{\text {th }}$ waveguides of both, left and right halves, is composed of two equal straight sections, with a length given by $(i-1) \Delta L / 2$, interconnected by a curve with an angle $\varphi=2 \arctan (\Delta L / 2 s)$ which is repeated in all waveguides. In this way, the lengths of the waveguides of the left and right parts can even be designed by imposing $m=0$, so that they do not contribute to the path length differences which, in this case, are introduced only by the central part. This design differs from Adar's design only by the fact that it makes use of identical bends. If desired, it is also possible to distribute the length differences over both, the lateral and central parts.

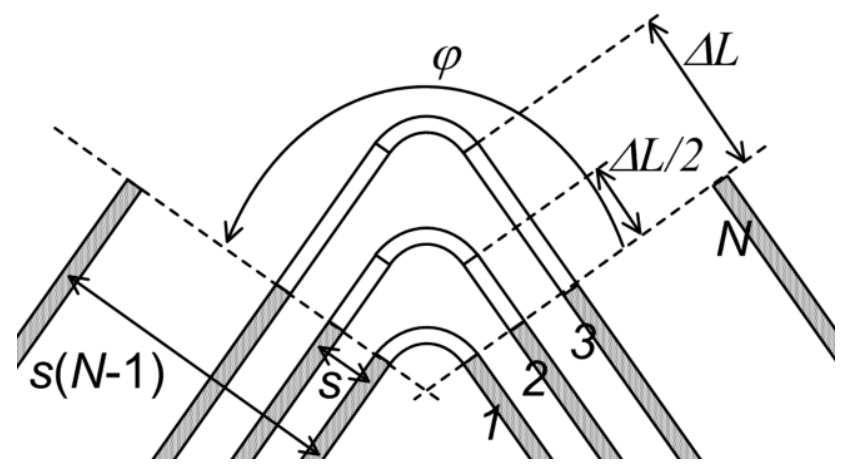

Fig. 5.4 - Interconnection between the left and right halves of the AWG for high-order designs. The terminal parts of the waveguides of the left and right halves are shown in gray, and are numbered from 1 to $N$.

One of the advantages of the identical-bend high-order layout over the conventional layout is that it can lead to a device with a smaller footprint. We illustrate this fact by comparing two designs of the same AWG, one making use of the identical-bend geometry and the other of the conventional geometry. For the design we chose waveguides with the same cross-section and refractive-index contrast as those described in the next Section. The following parameters were chosen for the AWG: central wavelength $\lambda_{\mathrm{c}}=881.53 \mathrm{~nm}$, wavelength spacing between the output channels $\Delta \lambda=0.01 \mathrm{~nm}$, order $m=5877$, minimum bending radius $r_{\min }=1700 \mu \mathrm{m}$, FPR radius $R=1126 \mu \mathrm{m}$, separation between the arrayed waveguides at the FPR $d=7.5 \mu \mathrm{m}$, and $N=31$ arrayed waveguides. The minimum bending radius was chosen to have negligible bending losses (below $2 \times 10^{-4} \mathrm{~dB} / \mathrm{cm}$ ) when taking into account the fabrication tolerances. For both layouts we optimized the geometrical parameters to obtain compact footprints. By calculating the area of the two designs (excluding the input and output channels) we obtain approximately $5.4 \mathrm{~cm}^{2}$ for the conventional layout and $3.4 \mathrm{~cm}^{2}$ for the identical-bend layout (an area reduction of $37 \%$ ). Despite the reduction in area, the identical-bend design 
suffers from being longer than the conventional design. The length difference in the analyzed case (with 31 arrayed waveguides) is only of a few millimeters, but increases linearly with the number of waveguides.

If the device size is reduced by use of smaller bending radii at the expense of increased losses, the identical-bend design does not incur the systematic phase errors that degrade the performance in the conventional design. In case the minimum bending radius is reduced to $850 \mu \mathrm{m}$, we obtain $4.5 \mathrm{~cm}^{2}$ area for the conventional design and $2.1 \mathrm{~cm}^{2}$ for the identical-bend design, corresponding to a reduction of the occupied area of 54\% (see Fig. 5.5). In this case the bending losses (calculated accounting for fabrication tolerances) are around $0.3 \mathrm{~dB} / \mathrm{cm}$, resulting in a total bending loss lower than $0.1 \mathrm{~dB}$ for the entire device. We simulated the effect of the systematic phase errors on these AWGs. Figure 5.6 (a) shows a comparison between the identical-bend design, in which the systematic phase errors are not present, and the conventional design. The phase errors in the conventional design produce a shift in the central wavelength of the channels (approximately $0.002 \mathrm{~nm}$ ), and a deformation in the passband shape. In Fig. 5.6 (b) we overlapped the response of one output channel of the identical-bend AWG with the response of the same output channel of the conventional AWG to show the effect of the phase errors on the passband shape. To better illustrate the difference in passbands the response of the conventional AWG was shifted by $0.002 \mathrm{~nm}$ such that the centers of the two passbands coincide.

The systematic phase errors for the two conventional AWGs with $r_{\min }=1700$ $\mu \mathrm{m}$ and $850 \mu \mathrm{m}$, respectively, are displayed in Fig. 5.7. The systematic phase errors are considered as the deviations of the phase difference between each two adjacent arrayed waveguides with respect to the intended value of $2 m \pi$ (at the central wavelength).

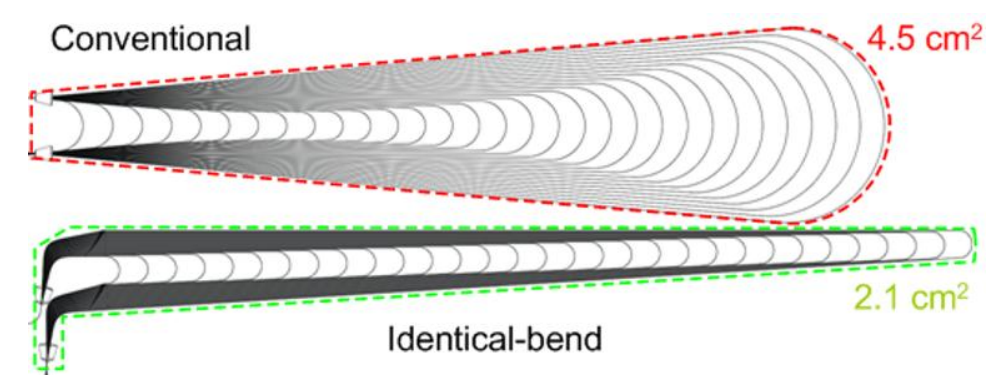

Fig. 5.5 - Comparison of the conventional and identical-bend layouts in terms of the occupied area. 

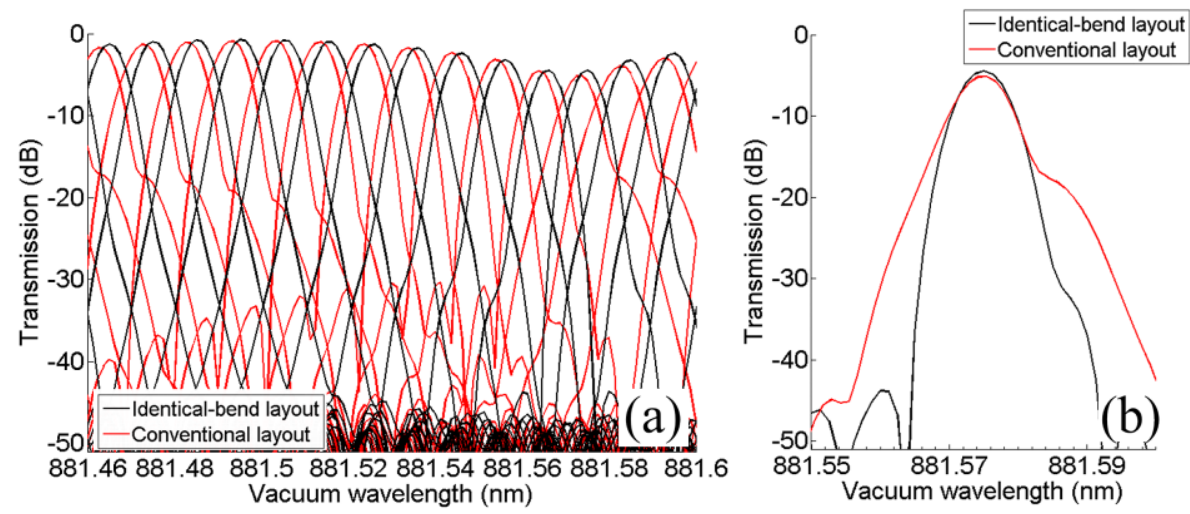

Fig. 5.6 - (a) Simulated effect of systematic phase errors on the response of an AWG designed with the conventional layout (red line). These phase errors are not present in the identical-bend layout (black line). Both graphs are for the transverse-electric (TE) polarization; (b) the response of an outer channel of the identical-bend AWG is overlapped with that of the same channel of the conventional AWG to show the difference in passband.

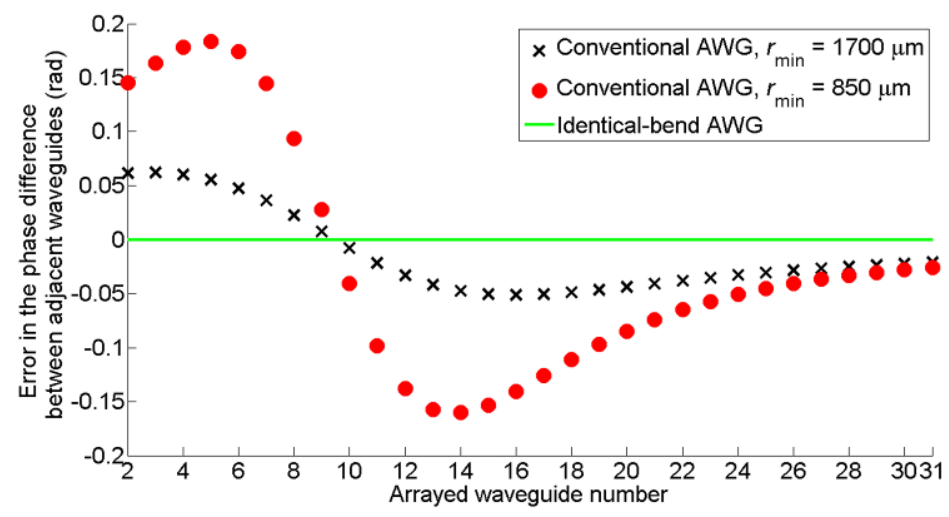

Fig. 5.7 - Calculated systematic phase errors between adjacent waveguides in a conventional AWG design for the transverse-electric (TE) polarization, and for two different values of the minimum bending radius rmin. These phase errors are not present in the identical-bend design, whatever value of the minimum bending radius is used (green line).

\subsection{Integrated spectrometer for the skin application}

One of the main outcomes of the Raman-pen project is the realization of compact integrated optical devices for in vivo confocal Raman spectroscopy of the skin, specifically for the detection of water concentration and the natural moisturizing factor (NMF) [Cas98]. For this purpose we propose the layout in Fig. 5.8 that combines the functions of light focusing and signal collection, together with suppression of Rayleigh backscattered light, and wavelength separation. For focusing the excitation laser light into the sample and collecting backscattered light we arrange two zero-order AWGs in a confocal setup, while for the suppression of 
backscattered laser light and wavelength separation of the Raman signal an integrated optical filter and an additional AWG, respectively, are used.

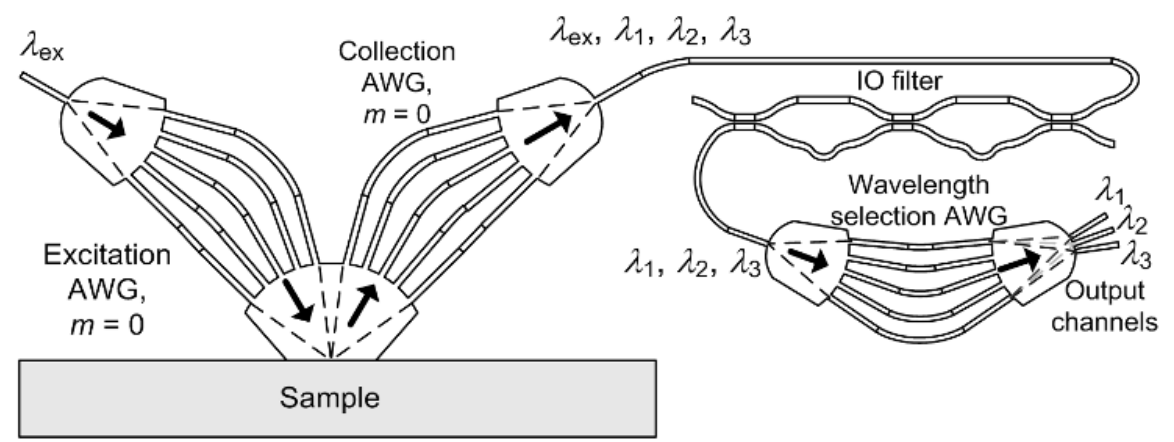

Fig. 5.8 - Schematic of the proposed device with the three distinctive parts: confocal arrangement of two zero-order $(m=0)$ AWGs for laser delivery and signal collection; an integrated optical filter for laser suppression; and an AWG for wavelength separation.

The configuration of Fig. 5.8 can be used, in principle, for both the skin applications by changing slightly the design parameters of the laser suppression filter and the wavelength selection AWG. In this Section we discuss the design and experimental characterization of the wavelength selection AWG for NMF detection. For the water application the designs are carried out in a similar way, and therefore, will not be addressed here. In Appendix B we present the design of the laser suppression filters based on the cascaded interconnection of Mach-Zehnder interferometers (MZIs). In the Appendix we also present preliminary experimental results carried out on the fabricated filters.

\subsubsection{Design and experimental characterization of a broadband AWG for skin NMF concentration detection}

Our experimental AWG device is designed for the analysis of Raman emission from human skin to determine its NMF. With laser excitation at a wavelength of 785 $\mathrm{nm}$ and a Raman shift between $700 \mathrm{~cm}^{-1}$ and $1700 \mathrm{~cm}^{-1}$, the wavelength range of interest is between 830 and $900 \mathrm{~nm}$. The application requires polarization insensitivity, a central wavelength of the AWG of $881 \mathrm{~nm}$ (corresponding to the spectral position of one of the NMF bands to be detected), and a minimum resolution of $5.5 \mathrm{~nm}$. Particular care must be taken to avoid overlap between the excitation wavelength - imaged at a higher order - and any of the output channels; in our particular case, this translates into the requirement of a minimum FSR greater than $115 \mathrm{~nm}$. To fulfill all these requirements, we designed a $3^{\text {rd }}$-order AWG using low-birefringence $\mathrm{SiON}$ channel waveguides with an $\mathrm{SiO}_{2}$ cladding. Higher grating orders would have resulted in the (backscattered) excitation wavelength overlapping with or falling close to one of the Raman channels, while smaller orders would have resulted in an increase of the dimensions of the AWG. The waveguides have core and cladding refractive indexes of 1.509 and 1.454, respectively, in TE polarization. The chosen waveguide geometry, a cross-section of $2 \mu \mathrm{m} \times 0.52 \mu \mathrm{m}$, results in low 
channel-waveguide birefringence $\left(\Delta n_{\text {eff,TM-TE }}<5 \times 10^{-4}\right)$ across the Raman band of interest. Although the birefringence in the bends has a slightly different value, this does not affect the response of the identical-bend AWG. When designing the AWG according to the proposed geometry, the angle $\alpha=49^{\circ}$ was chosen to minimize the size of the device, while $\Delta \alpha \cong 0.1^{\circ}$ was fixed once the FPR length $R \cong 4 \mathrm{~mm}$ and the separation $d=7.5 \mu \mathrm{m}$ between the arrayed waveguides at the FPR interface were determined. The latter two parameters are related through the AWG design equations [Smi96] to the output channel wavelength spacing $\Delta \lambda$, which in our case is $5.5 \mathrm{~nm}$. Furthermore, we chose a relatively large number of arrayed waveguides $(N=131)$ as a compromise between minimizing the spillover losses and having a reduced overall device size. The bends used in our implementation are cosine bends, where the curvature changes continuously from a value of zero at the input and output ports, reaching a maximum in the center of the bend. The minimum bending radius for the type 1 bend was $1750 \mu \mathrm{m}$ to guarantee bending losses below 0.1 $\mathrm{dB} / \mathrm{cm}$ (calculated accounting for fabrication tolerances) at the highest wavelength in the spectral region of interest. This choice of bending radius resulted in a bend length of $2500 \mu \mathrm{m}$. Since the type 2 bend is repeated many times, the change in curvature across each interconnected bend translates into a periodicity of the effective index of the guided mode. Hence, for the bend of type 2, we chose a much larger minimum bending radius of $5100 \mu \mathrm{m}$ to obtain a negligible change in the effective index $\left(\Delta n_{\text {eff }} \sim 2.1 \times 10^{-6}\right)$ across the bend. This choice resulted in a bend length of $15 \mu \mathrm{m}$. The device was fabricated using an e-beam written mask having a minimum resolvable feature size of $0.7 \mu \mathrm{m}$, and a defect density of 0.64 defects/inch ${ }^{2}$ with maximum defect size of $2 \mu \mathrm{m}$. As mentioned in the introduction, the design is based on an arrangement of the arrayed waveguides with a constant angular spacing. For this reason the condition of equal chord projections required by the Rowland mounting is not met. However, the maximum deviation of arrayed waveguide positions at the grating line from the Rowland mounting configuration is about $0.16 \mu \mathrm{m}$. Not only is this value much smaller than the e-beam mask resolution, it is also much smaller than the waveguide width. Therefore, in this case, our layout is a good approximation of the Rowland mounting geometry.

For device characterization we used the setup shown in Fig. 5.9. Light from a Fianium super-continuum source (from $400 \mathrm{~nm}$ to $1800 \mathrm{~nm}$ ) was sent through a polarization beam splitter and a red-glass filter (RG715) to suppress the undesired region of the spectrum $(400-715 \mathrm{~nm})$. The light was then focused into the input waveguide of the AWG by a microscope objective with a numerical aperture (NA) of 0.65 and a magnification of $\times 40$. The spectral response of the AWG was measured by coupling each output channel to a spectrometer (iHR550 Horiba) through a single-mode fiber. The input and output slit widths of the spectrometer were adjusted to the same value of $0.1 \mathrm{~mm}$ and the measurement resolution was 0.25 nm. 


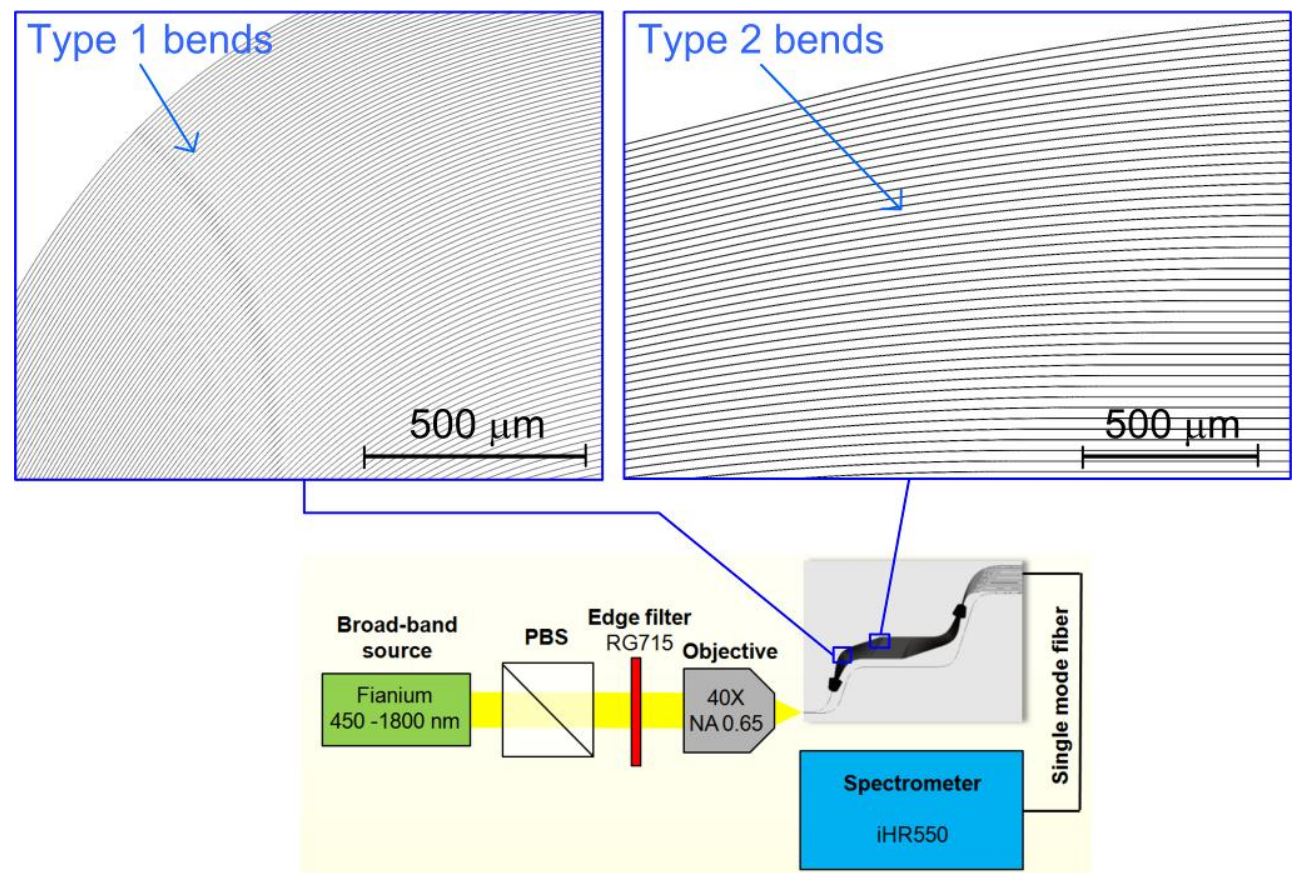

Fig. 5.9 - Setup used to characterize the AWG. PBS = polarizing beam splitter. In the insets we present two enlarged views of the AWG layout pointing at the locations of the bends of type 1 and 2 .

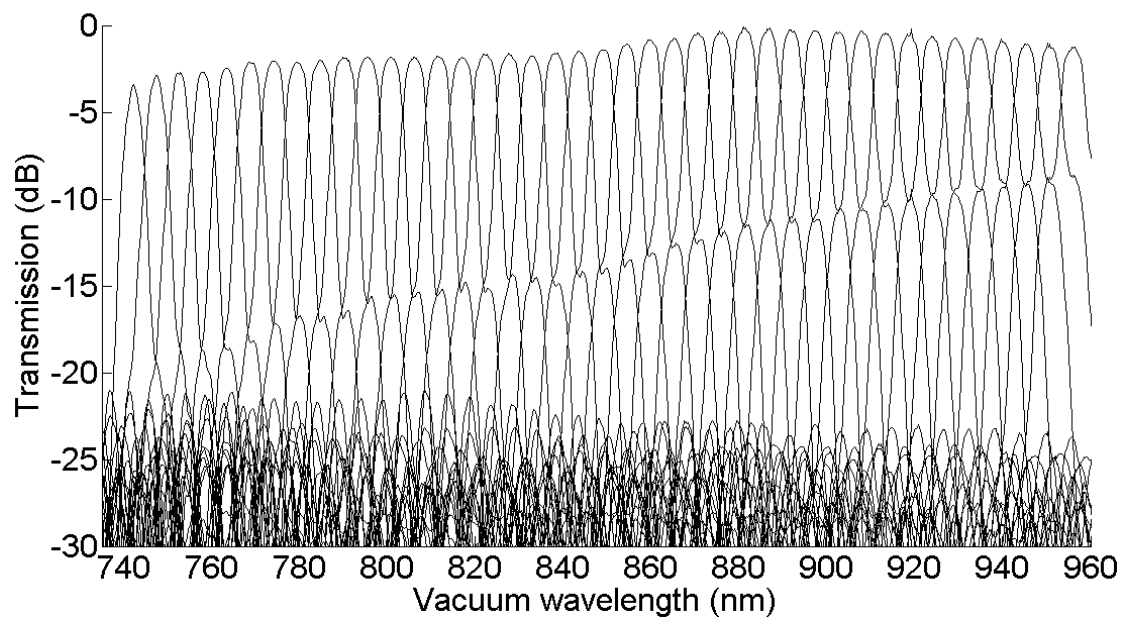

Fig. 5.10 - Measured spectral response of the AWG for TE polarization.

The measured response was normalized with respect to the spectrum from a separate reference channel on the same chip and is shown for TE polarization in Fig. 5.10. As can be observed, the intrinsic losses (excluding coupling losses and propagation losses) of the AWG are very low, ranging from $1.1 \mathrm{~dB}$ for the central 
channel up to a maximum of $1.8 \mathrm{~dB}$ at the edges of the spectral region of interest (830 to $900 \mathrm{~nm}$ ). We estimate an error in the measured loss value of $\sim 2 \mathrm{~dB}$ since it is not possible to achieve the same coupling conditions in all the output channels as those for the reference waveguide. We also measured the total transmission through the device (from input fiber to output fiber) to be $9.6 \%$ at a wavelength of $832 \mathrm{~nm}$. The fiber-to-chip coupling efficiency was estimated to be $70 \%$ per facet using mode overlap calculations; the remaining $40 \%$ loss is due to propagation losses.

Figure 5.11 displays the normalized responses for TE and TM polarizations measured for 5 central and 11 outer channels of the device.
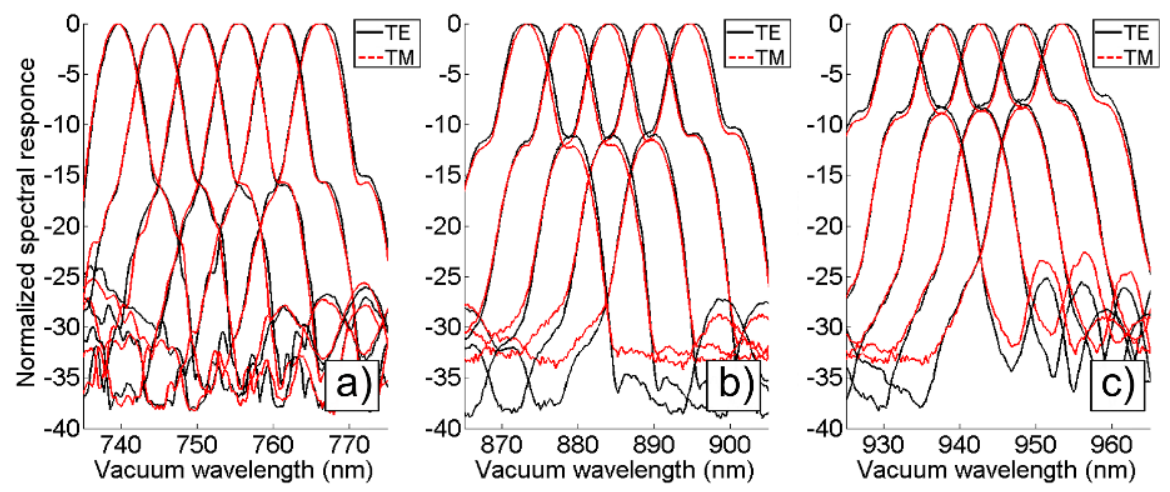

Fig. 5.11 - Normalized spectral response of the AWG measured for both, TE and TM polarizations, and for three different spectral regions: a) $740-770 \mathrm{~nm}$; b) $870-900 \mathrm{~nm}$; c) 930-960 nm.

The measured FSR is $215 \mathrm{~nm}$, and the device is polarization insensitive over the whole spectral range, the maximum shift between TE and TM polarizations being $0.5 \mathrm{~nm}$, which is more than 10 times smaller than the resolution.

In Figs. 5.10 and 5.11 we observe that for all the output channels of the AWG two shoulders are present next to the main peak. We also observe that the intensity of the shoulders increases with wavelength. The shoulders can also be observed, although with lower intensity, in the 2D beam-propagation-method (BPM) simulation shown in Fig. 5.12(a). The simulation is phase error free, so the shoulders are not caused by phase errors in the design. 

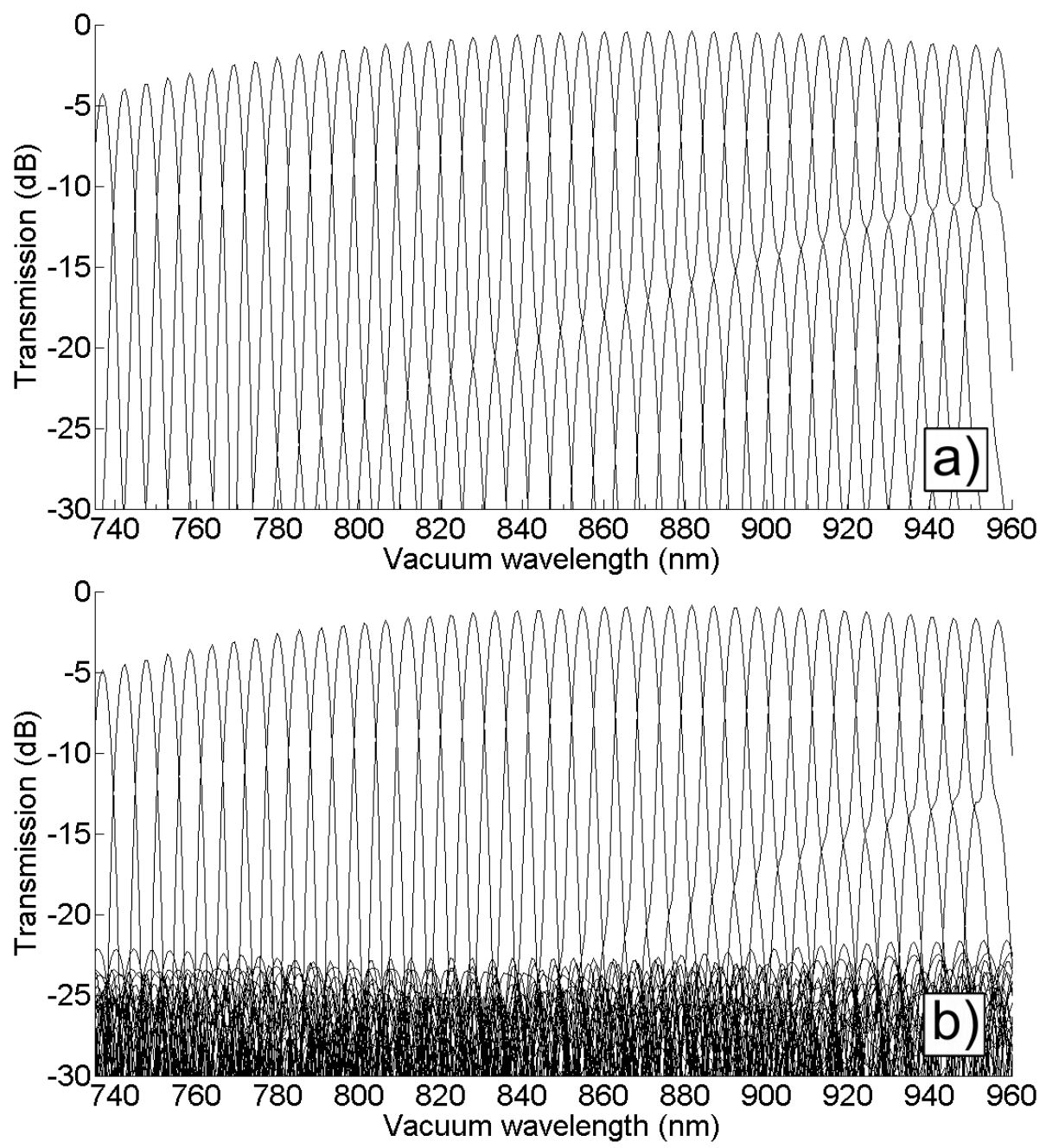

Fig. 5.12 - Simulated spectral response for TE polarization using the $2 \mathrm{D}$ beampropagation-method (BPM) (a) with no phase errors and (b) with random phase errors distributed between 0 and 80 degree.

We attribute the shoulders to coupling between the output-channels of the AWG which are initially spaced by $6 \mu \mathrm{m}$ and then separate from each other at an angle of $\sim 0.086$ degrees. The small separation distance was chosen in the design phase as a compromise between increased crosstalk due to coupling and reduced size of the device and therefore lower propagation losses. We preferred low losses and higher crosstalk since for our target application (Raman sensing) low losses are a must while there are no stringent specifications on the crosstalk. We also performed 2D BPM simulations (see Fig. 5.12 (b)), adding random phase errors (distributed in the interval $0-80$ degrees) in all the arrayed waveguides, to show how the effect of these errors increases the noisy background to around $-25 \mathrm{~dB}$, which is similar to what we observe in the measurement. 
The fabricated device has dimensions of $5 \mathrm{~cm}$ by $2 \mathrm{~cm}$, occupying a large area on the wafer, and for this reason the variations in the material properties and layer thickness across the wafer cause phase errors. These can be seen by comparing Fig. 5.10 and Fig. 5.12. We notice that the peak width in the fabricated device is larger than in the simulations and that there is a small shift in the central wavelengths of the external channels with respect to the simulated values. The presence of these fabrication-related phase errors does not enable us to demonstrate the cancellation of the systematic phase errors arising from the use of different bends in the fabricated device.

\subsection{Integrated spectrometer for the dental application}

In recent years researchers have studied and demonstrated the possibility of using Raman spectroscopy as a non-invasive tool for the detection of dental caries [Hil00], and new techniques such as polarized Raman spectroscopy have been proposed for detecting dental caries at an early stage [Ko06].

These methods rely on measuring the Raman signals from tooth enamel within the fingerprint region which extends from approximately $375 \mathrm{~cm}^{-1}$ to $1175 \mathrm{~cm}^{-1}$. In this region the strongest band is the symmetric $\mathrm{P}-\mathrm{O}$ stretching vibration of the phosphate ions $\left(\mathrm{PO}_{4}{ }^{3-}\right)$ within the hydroxyapatite crystal of tooth enamel, which is located near $959 \mathrm{~cm}^{-1}$. Changes in the intensity of the hydroxyapatite peak with respect to the luminescent background $930 \mathrm{~cm}^{-1}$ have been used as a measure of the state of the disease [Hil00]. Alternatively, in the case of polarized Raman spectroscopy, information on the state of the disease is provided by comparing the intensities of two orthogonal polarization states of the $959 \mathrm{~cm}^{-1}$ hydroxyapatite peak. The relevant polarization states are perpendicular and parallel to the polarization of the excitation laser, and their intensity ratio will be referred to as the depolarization ratio, $\rho_{959}=I_{959}(\perp) / I_{959}(\|)$. In sound enamel the hydroxyapatite signal is highly polarized in the direction parallel to that of the excitation laser, and the value of the depolarization ratio is approximately $\rho_{959}=0.10 \pm 0.04$ [Ko06]. As the carious lesion develops, there is a gradual structural modification of the tooth enamel, which also affects its optical properties, causing $\rho_{959}$ to increases to $\sim 0.4 \pm 0.12$ [Ko06].

The integrated device that we propose for performing polarized Raman spectroscopy on teeth is schematically shown in Fig. 5.13. The components are the same as those used in the spectrometer for NMF detection described in Section 5.3 (see Fig. 5.8), except that here an on-chip polarization splitter is introduced to separate the two polarization components of the collected light. Then as before each component is propagated through an integrated laser suppression filter and a wavelength selection AWG. An alternative, but more challenging, configuration can be achieved placing only one polarization-independent laser suppression filter before the polarization splitter instead of the two laser suppression filters shown in Fig. 5.13. For this dental application, the wavelength selection AWGs should have a 
higher resolution $(0.2 \mathrm{~nm})$ than for the skin application discussed in 5.3. In this case we chose the conventional horse-shoe layout due to the high order of the AWG.

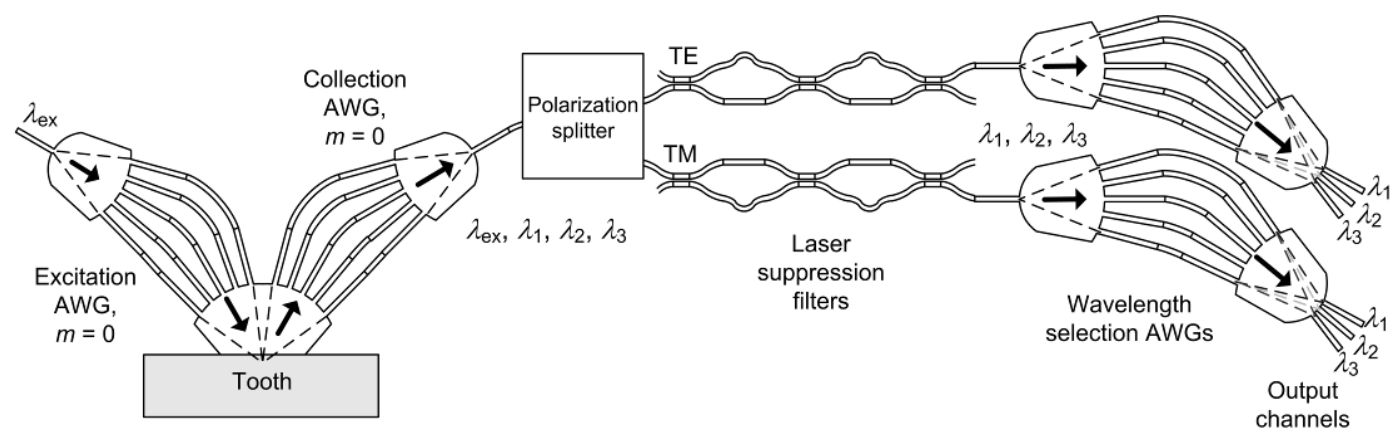

Fig. 5.13 - Schematic layout of the proposed integrated optical device for polarized Raman spectroscopy of teeth.

In this Section we discuss the design and experimental characterization of the wavelength selection AWG, while for the polarization splitter we address the reader to Appendix C, where we propose a novel design for the polarization splitter based on the arrayed waveguide grating.

\subsubsection{Design and experimental characterization of the AWG for dental caries detection}

The wavelength selection AWG (see Fig. 5.13) was designed to be used for detecting the Raman signal from teeth, using an excitation wavelength of $\lambda_{0}=830$ $\mathrm{nm}$. The reason we chose this wavelength is because it induces less fluorescence background in tissue than shorter wavelengths and also to have a direct comparison of our results with those obtained by our project partners (reported in Ref. [Ko06]), which make use of the same wavelength. This choice of the excitation wavelength positions the hydroxyapatite peak at $\sim 901 \mathrm{~nm}$. In order to completely resolve this peak, as well as part of the fluorescent background surrounding it, we designed the wavelength-selective AWG to have a central wavelength of $901 \mathrm{~nm}$, a resolution of $\Delta \lambda=0.2 \mathrm{~nm}$ and a grating order of $m=41$. The particular grating order was chosen to obtain a FSR of $22 \mathrm{~nm}$ (between 890 and $912 \mathrm{~nm}$ ); in this case the (backscattered) laser wavelength is imaged at a higher order $(m=45)$ on the outer channels of the device. Smaller orders would have resulted in larger dimensions of the AWG.

The AWG was fabricated using single-mode SiON channel waveguides with 2.2 $\mu \mathrm{m}$ width and $0.52 \mu \mathrm{m}$ height. The waveguide core and cladding refractive indices were 1.509 and 1.445 at $830 \mathrm{~nm}$, respectively. The center-to-center spacing $d$ between the $N=271$ arrayed waveguides at the FPR interface was $6 \mu \mathrm{m}$, and so was the spacing between the output channels of the AWG. From these parameters one can calculate the dispersion angle $\vartheta$ of the AWG as in Eq. (2) of [Smi96], which we repeat here for convenience: 


$$
\vartheta \approx \frac{\beta(\lambda+\Delta \lambda) \Delta L-m 2 \pi}{\beta_{F P R}(\lambda+\Delta \lambda) d} .
$$

From this angle the length $R$ of the FPR can be determined as $R=d / \sin (\vartheta) \cong 6.4$ $\mathrm{mm}$. The arrayed-waveguide layout was drawn by imposing a minimum bending radius of $1700 \mu \mathrm{m}$ to guarantee bending losses below $0.1 \mathrm{~dB} / \mathrm{cm}$ when taking into account fabrication tolerances. The simulated spectrum of the device is shown in Fig. 5.14.

We characterized the fabricated AWG using the setup shown in Fig. 5.15. TEpolarized light from a broadband source (Fianium) was coupled to the AWG with a polarization-maintaining (PM) fiber. Several central and outer channels were characterized by measuring the output spectrum and normalizing it with respect to a reference channel.

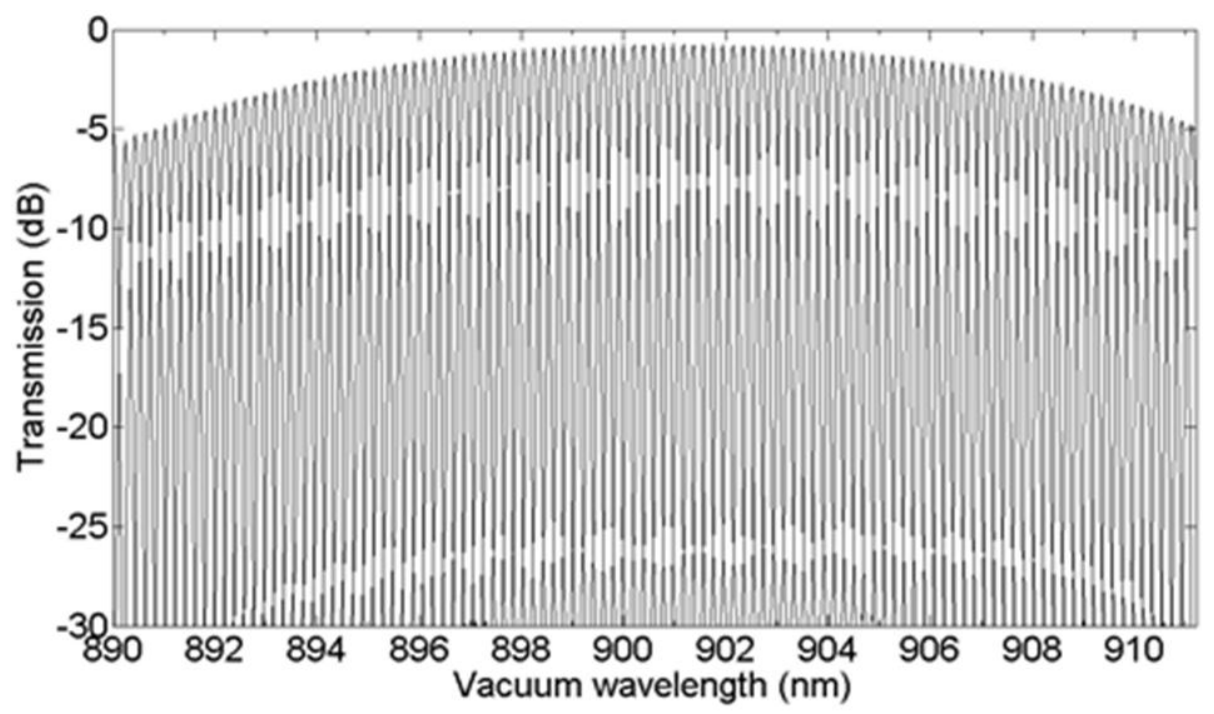

Fig. 5.14 - Simulated spectral response for TE polarization using the 2D beampropagation-method (BPM) of the AWG for dental caries detection. 


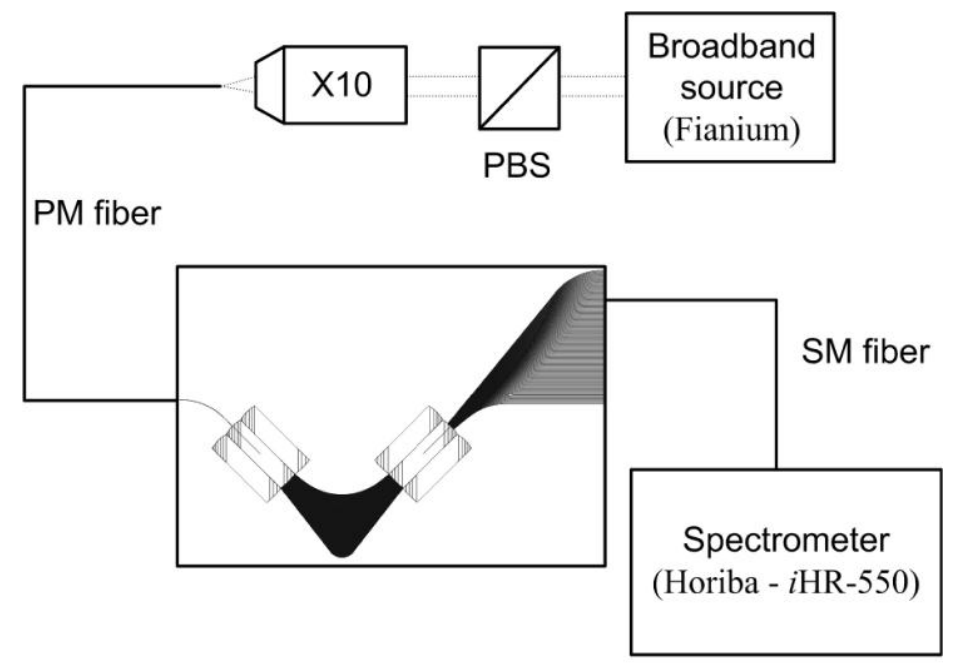

Fig. 5.15 - Setup used to characterize the AWG. PM fiber = polarizing maintaining fiber, $\mathrm{SM}$ fiber $=$ single mode fiber, $\mathrm{PBS}=$ polarization beam splitter.

The spectral responses of five central channels are shown in Fig. 5.16(a), where they are compared with the simulated responses of the same channels shown in Fig 5.16(b). The characterized device has a central wavelength of $900.78 \mathrm{~nm}$ (for TE polarization), a resolution of $0.2 \mathrm{~nm}$ and a FSR of $\sim 22 \mathrm{~nm}$ (as designed). The central output channels have a $3-\mathrm{dB}$ bandwidth of $0.11 \mathrm{~nm}$, while for the outer channels we obtained a bandwidth of $0.13 \mathrm{~nm}$. The AWG adjacent channels have a crosstalk of $\sim 10 \mathrm{~dB}$, and the intrinsic losses of the device (compared to a reference waveguide) are $\sim 2 \mathrm{~dB}$ for the central wavelength and $\sim 7 \mathrm{~dB}$ for the outer channels.
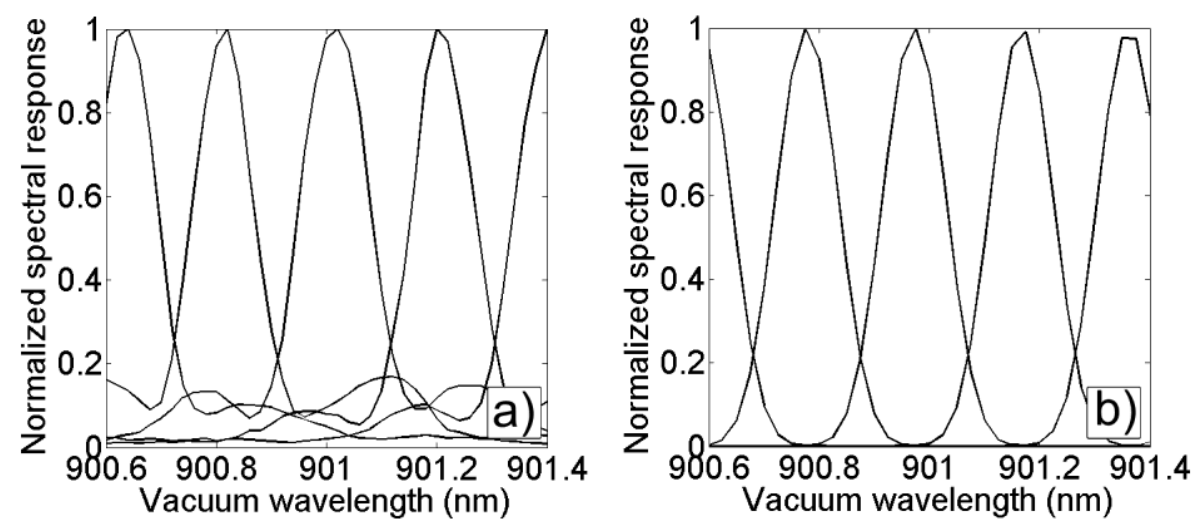

Fig. 5.16 -Normalized spectral response of the central channels of the AWG for the transverse-electric (TE) polarization. (a) measured; (b) simulated.

The characterized AWG exhibited a polarization-dependent response in which the wavelength shift between the TE and the TM polarizations was $0.5 \mathrm{~nm}$. 


\subsection{Summary}

Detailed designs of Raman spectrometers were discussed. In particular, we presented the designs of spectrometers for the detection of the skin's natural moisturizing factor and early dental caries.

We started by presenting a novel layout for the design of AWGs of any diffraction order. The proposed geometry makes use of identical bends across the entire grating, leading to a complete cancellation of systematic phase errors which are intrinsically present in conventional designs. Another advantage of this layout is that it occupies a smaller area compared to the conventional horse-shoe AWG. In particular, our design allows us to reduce the device size more than it would be possible with a conventional geometry without incurring a significant distortion of the AWG response due to the increase of the systematic phase errors at smaller bending radii. Furthermore, we presented the design steps and the results of the characterization of the NMF detection AWG fabricated according to the proposed layout. The AWG presented low losses and polarization insensitivity over a wide spectral range of $215 \mathrm{~nm}$ in the near-infrared spectral region.

The last Section was dedicated to the spectrometer for the dental application. We showed the design and characterization of the wavelength selection AWG, which performed as expected, presenting a high resolution of $0.2 \mathrm{~nm}$, a central wavelength of $901 \mathrm{~nm}$ and a FSR of $22 \mathrm{~nm}$. 


\section{Chapter 6}

\section{Integrated Raman spectroscopy}

\subsection{Introduction}

I

$\mathrm{n}$ this Chapter we discuss the use of the arrayed waveguide grating that we designed for the dental application and that was described in Chapter 5, to measure polarized Raman spectra from a sample of cyclohexane and from extracted human teeth exhibiting sites with early dental caries.

As already mentioned in the previous Chapter, when motivating the choice of the AWG for Raman spectroscopy, one of its advantages over conventional spectrometers is its small size $(\sim \mathrm{cm} 2)$ and therefore, the possibility to be integrated in a handheld device. On the other hand, a potential disadvantage is the limited free spectral range (FSR) achievable for a given device size which can, however, be overcome by a technology platform providing larger refractive index contrast, e.g. silicon photonics, or by accepting spectrally folded, yet non-overlapping peaks, as demonstrated in one of the two examples presented in this Chapter.

In both the presented examples the Raman spectra obtained with our device are compared with the measurements obtained using conventional bulk spectrometers performed by our project partners in the Institute of Biodiagnostics of the National Research Center of Canada. As we will show in the next Sections the measurements results are in excellent agreement.

\subsection{Arrayed waveguide grating based Raman spectroscopy}

For the polarized Raman measurements on tooth samples we used the setup shown in Fig. 6.1. Light from a linearly polarized, tunable Ti:Sapphire laser at $830 \mathrm{~nm}$ was sent through a first polarization beam splitter (PBS) oriented parallel to the laser polarization which was parallel to the optical table. A half-wave plate was positioned after the PBS, initially with one of its optical axes parallel to the laser polarization, so that the polarization was not rotated. The laser light exiting the $\lambda / 2$ plate, after passing through a laser-line filter (Semrock LL01-830-12.5), was reflected from a dichroic mirror (Semrock LPD01-830RS-25) and focused onto the sample with a microscope objective. The light backscattered from the sample was then collected by the same optics; the Rayleigh component was again reflected (rejected) by the dichroic mirror, which transmitted the Raman wavelengths. An edge filter (Semrock LP02-830RS-25) and an additional red-glass filter (RG 850) were used to further suppress residual light at the laser wavelength. At this point the light passed through another PBS with the same orientation as the first PBS, and was focused onto the input channel of the integrated AWG spectrometer by a $\times 50$ 
microscope objective with numerical aperture NA $=0.85$. The output channels of the AWG were imaged onto an electron-multiplying charge-coupled device (Andor iXonEM DV887ECS-BV, EMCCD) through a camera lens (JML f0.95 $50 \mathrm{~mm}$ ).

In order to perform the measurements for cross polarization, we rotated the $\lambda / 2$ plate by $\sim 45^{\circ}$, so that the polarization of the excitation laser was rotated by $90^{\circ}$. The extinction ratio (in transmission) of the PBSs was measured to be around $0.2 \%$. In both, parallel and cross-polarization measurements light entering the AWG was always horizontally polarized (TE polarization with respect to the input waveguide). Thus, there was no need to calibrate for the polarization dependence of the AWG.

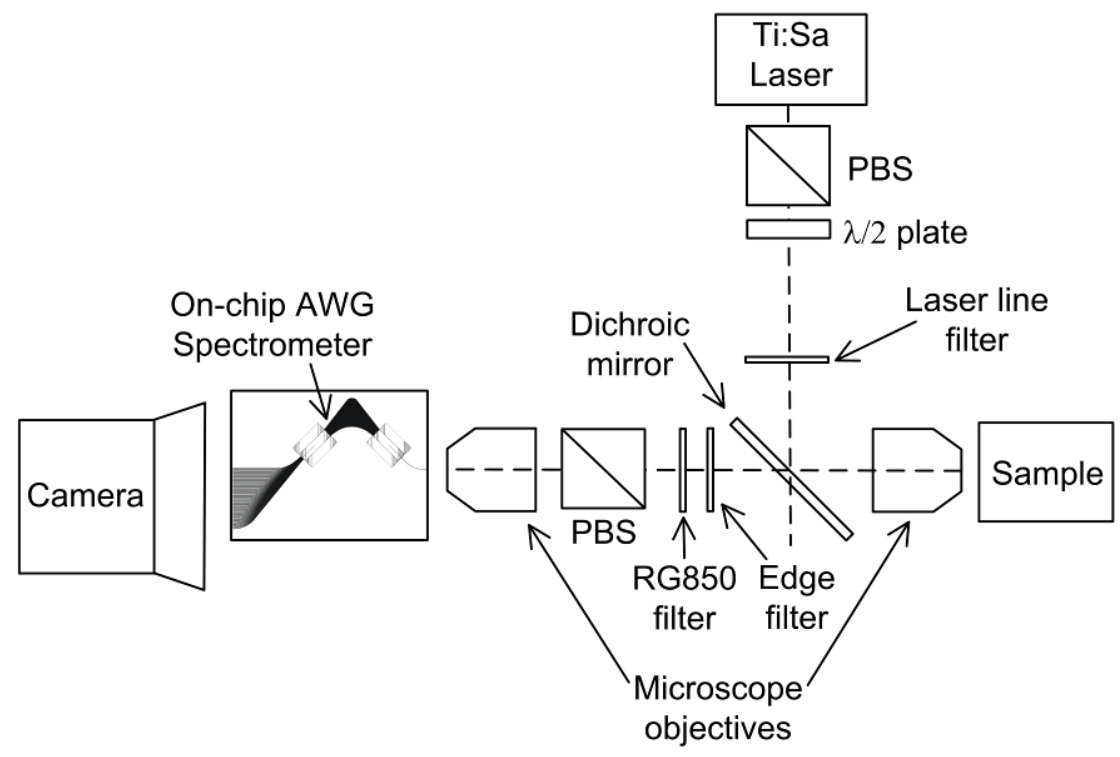

Fig. 6.1. Setup used for the polarized Raman experiments.

\subsubsection{Polarized Raman spectroscopy on cyclohexane}

Before performing the PRS measurements on tooth samples we carried out initial test measurements with our AWG on cyclohexane, chosen for its well-known and documented Raman spectra for both polarizations [Fen47, Gar85]. The inset of Fig. 6.3 displays the unpolarized Raman spectrum of cyclohexane in the fingerprint region (400-1600 $\mathrm{cm}^{-1}$ ), as measured using a bulk Raman spectrometer (based on a Model 2500 High Performance Raman Module, River Diagnostics B.V.). With the excitation wavelength of $830 \mathrm{~nm}$ chosen for the test measurements, the Raman signals of cyclohexane occur in the wavelength range between $859 \mathrm{~nm}$ and $957 \mathrm{~nm}$. Since this wavelength range exceeds almost fivefold the FSR of $22 \mathrm{~nm}$ (at the central wavelength $\lambda_{\mathrm{c}}$ ) of our AWG, the different parts of the Raman spectrum fall into five different diffraction orders ( $m=39,40,41,42$, and 43) of the AWG, as indicated in the inset. The same Raman spectrum, with the different diffraction orders folded into the diffraction order $m_{A W G}=41$, for which the AWG was 
designed, is calculated with the AWG grating equation [Tak95] and shown as a red line in Fig. 6.3. According to this equation a peak at wavelength $\lambda$ that falls into a diffraction order $m$ is imaged by the AWG at the same output location of a peak at wavelength $\lambda+\Delta m \times$ FSR that falls in the diffraction order $m_{\mathrm{AWG}}$, where $\Delta m=m-$ $m_{A W G}$, and the FSR is wavelength dependent [Tak95]. The individual peaks in the original and folded spectrum are assigned by letters $(\mathrm{a}-\mathrm{g})$.

In Fig. 6.3 we also show the Raman spectrum of cyclohexane as measured from our AWG spectrometer for both, parallel (triangles) and cross (dots) polarizations with an integration time of $80 \mathrm{~s}$ (the standard deviation is $\sim 5 \%$ ). For this measurement cyclohexane was placed in a quartz cuvette and the excitation laser was focused through the cuvette wall with a $\times 20$ microscope objective with $\mathrm{NA}=$ 0.6 .

In Fig. 6.3 the horizontal axis is given in wavenumber shift relative to the excitation wavelength of $830 \mathrm{~nm}$, and for the diffraction order $(m=41)$ for which the AWG was designed.

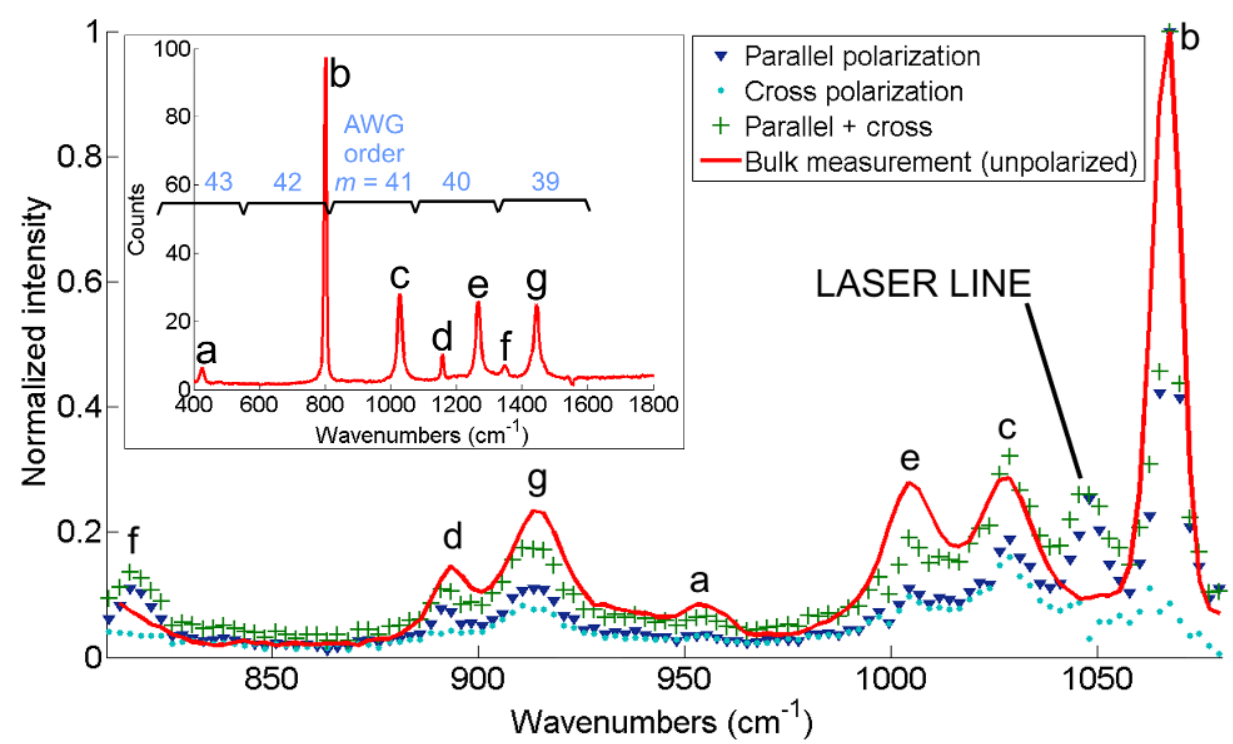

Fig. 6.3. Normalized Raman spectra of cyclohexane measured with the arrayedwaveguide-grating (AWG) spectrometer for the parallel (triangles) and cross (dots) polarizations. The sum of the two spectra (crosses) is compared with the unpolarized Raman spectrum of cyclohexane measured with a conventional spectrometer (red line) and folded over the different diffraction orders $(39,40,41,42$, and 43). The inset shows the original unfolded spectrum. For comparison, the Raman peaks in both, folded and unfolded spectra are indicated by letters ( $\mathrm{a}, \mathrm{b}, \mathrm{c}, \mathrm{d}$, e, f, and g). An additional peak is present, which is not related to cyclohexane. We attribute this peak to the laser line imaged at a higher order $(\mathrm{m}=45)$.

Comparison of the normalized sum (green crosses) of the two measured signals with the unpolarized spectrum (red line) of cyclohexane, as measured using the bulk 
spectrometer and folded into the diffraction order of the AWG, shows very good qualitative agreement: in Fig. 6.3 we clearly observe the presence of the strongly polarized Raman bands at the locations of peaks $b$ and $d$, in agreement with the results found in the literature [Pe199]. Furthermore, we observe that all the peaks of cyclohexane in the fingerprint region can be clearly distinguished.

This test result demonstrates that a measurement with an AWG spectrometer can unambiguously identify the Raman fingerprint of a measurand, even if its Raman spectrum exceeds the FSR of the AWG, thus allowing us to design and apply an AWG with high spectral resolution.

\subsubsection{Detection of early dental caries through polarized Raman spectroscopy}

As we explained at the beginning of Section 6.2, we used introduced an additional red-glass filter (RG850) in order to suppress light at the excitation wavelength entering the AWG. The choice to include this filter came after we conducted a set of preliminary un-polarized Raman measurements on tooth samples (see Fig. 6.2) were we could observe strong backscattering and reflection of the laser light that, entering the AWG, was imaged at a higher order on the lateral output channels. The sharp peak at $\sim 1050 \mathrm{~cm}^{-1}$ is attributed to a laser line artifact imaged at higher order $(m=$ 45).

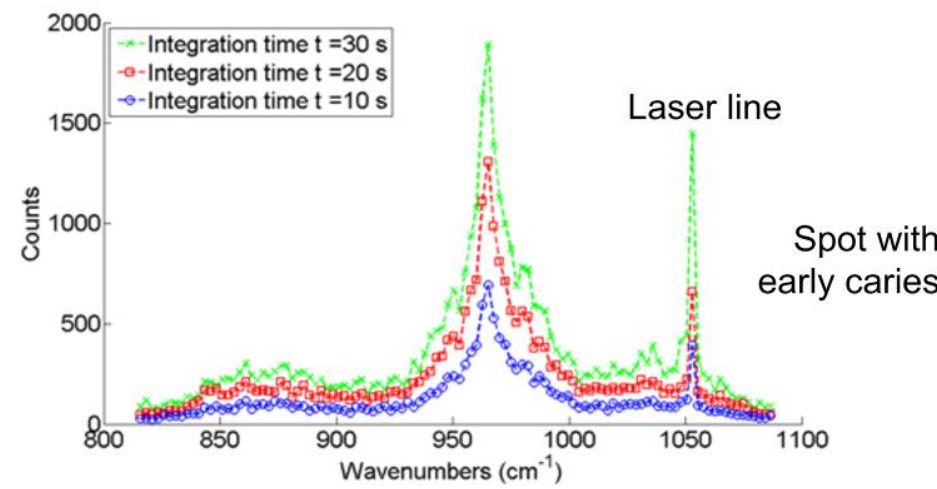

(a)

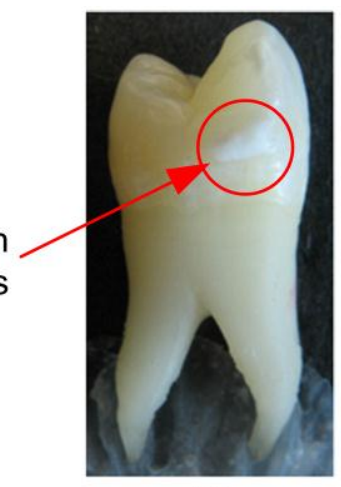

(b)

Fig. 6.2. a) Unpolarized Raman spectra of a tooth sample for three different values of the integration time; b) A tooth samples exhibiting an initial carious lesion (white spot).

Polarized Raman measurements on two extracted human tooth samples presenting clinical white spot lesions as early dental caries were also performed. We used a $\times 40$ microscope objective with an NA of 0.65 for focusing $200 \mathrm{~mW}$ of excitation light at a wavelength of $830 \mathrm{~nm}$ onto the surface of the tooth and collecting the backscattered light. The measurements were carried out on three healthy and three carious locations on the tooth surface which were chosen based 
upon previous visual examination by a dental clinician. The sites which presented initial carious lesions had a slightly whiter color with respect to the rest of the tooth surface and, therefore, could be distinguished by eye (see Fig. 6.2 (b)). The Raman signal from these regions was strong and clearly visible with integration times as short as $10 \mathrm{~s}$.

Representative polarized Raman spectra acquired from sound and carious enamel are shown in Fig. 6.3. These spectra are measured with $80 \mathrm{~s}$ integration time and 10 accumulations and have a standard deviation of $\sim 3 \%$. The trends of the spectral intensities at $959 \mathrm{~cm}^{-1}$ are consistent with polarized Raman spectra acquired using a conventional Raman microspectrometer (LabRamHR, Horiba Jobin Yvon) from the same tooth samples (see inset). In each of the six regions, we calculate the depolarization ratio and obtain an average value of $\rho_{959}=0.16 \pm 0.047$ for the sound regions and $\rho_{959}=0.41 \pm 0.01$ for the carious regions. These numbers are close to the values reported in the literature [Ko06]. The depolarization ratios are calculated using the peak intensities at $959 \mathrm{~cm}^{-1}$ relative to the adjacent baseline.

In Fig. 6.3 (b) the laser line signal seems to be almost double in intensity with respect to that in Fig. 6.3 (a), however this is partially because the measured height of the hydroxyapatite peak (in terms of counts) in the case of carious measurements is lower (4940 counts) with respect to that in the sound measurement (7550 counts). Also the surface of the tooth is irregular, which might cause strongly positiondependent reflections and back-scattering coefficients.

\subsection{Summary}

An AWG spectrometer has been characterized and used to measure polarized Raman spectra of cyclohexane and tooth samples.

Our experiments on cyclohexane demonstrated successfully that the AWG can be used in measuring spectra that extend beyond its FSR, as long as the peaks present in the original spectra do not overlap once folded into the diffraction order of the AWG. The results of polarized Raman spectroscopic measurements performed on cyclohexane were in good agreement with those found in the literature, therefore, validating our measurement setup. At this point the setup was used for similar measurements on extracted human teeth presenting sites with early dental caries. The results of these experiments demonstrated the feasibility to obtain Raman signals of the prominent hydroxyapatite peak at $\sim 959 \mathrm{~cm}^{-1}$ for both polarizations and that the depolarization ratios measured with our device are comparable with the values found in the literature for both, sound and carious regions of tooth enamel.

Our results pave the way towards the realization of complete systems based on integrated optics for the detection of early dental caries with polarized Raman spectroscopy. 

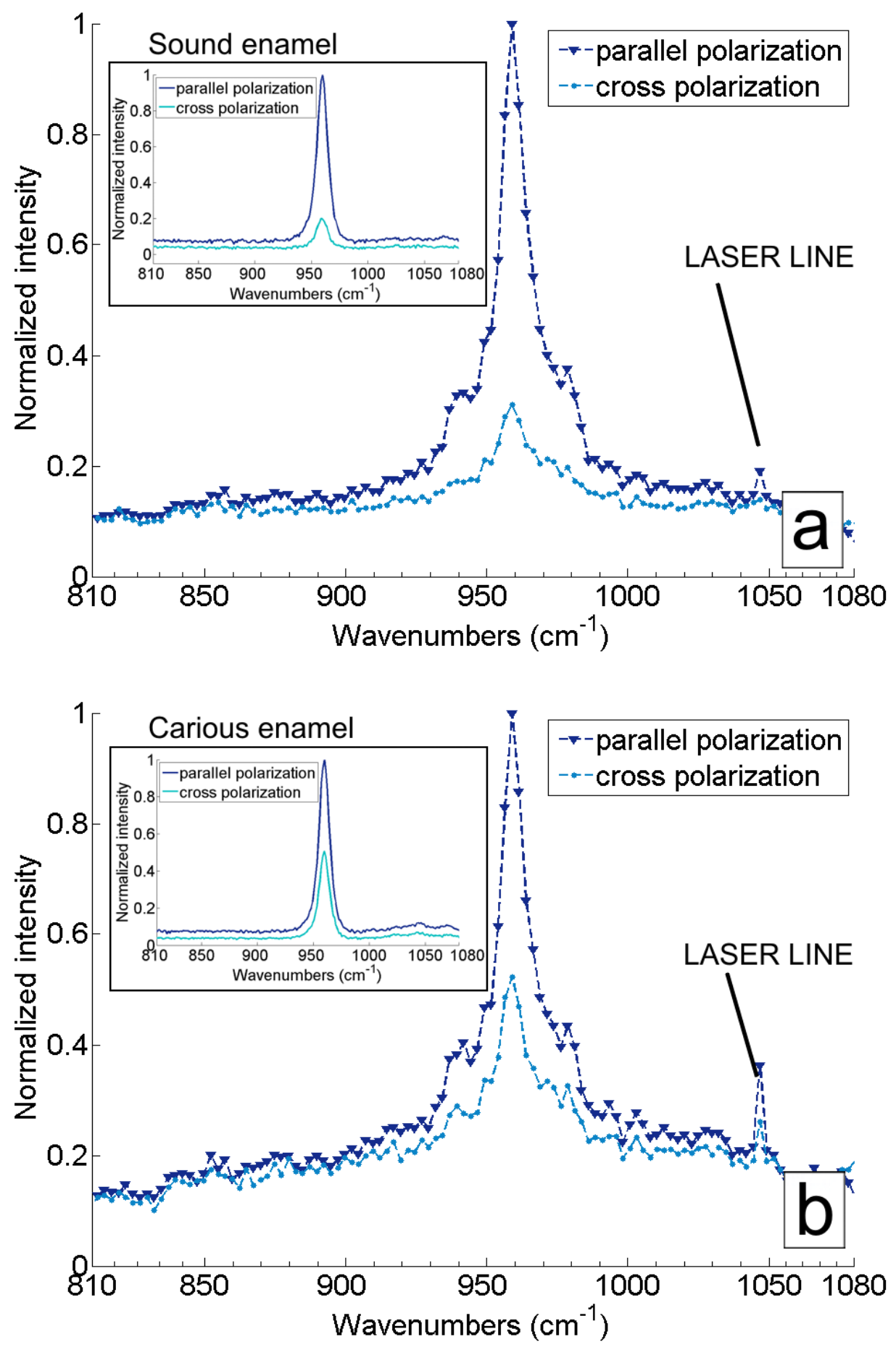

Fig. 6.3. Raman spectra of (a) sound and (b) carious tooth enamel for both, parallel (triangles) and cross (dots) polarization. The insets show spectra acquired with a conventional Raman microspectrometer. 


\section{Chapter 7}

\section{Conclusion and outlook}

$\mathrm{I}$ $\mathrm{n}$ the following we give a brief summary of the main results of our research. We also discuss the ways in which future research could benefit from our results by indicating the areas where improvements can be made and by suggesting ways to overcome some of the limitations that we encountered.

In the course of our research we have developed a novel device for laser delivery and confocal signal collection that may open the doors to new types of applications in the fields of microscopy and spectroscopy, for example in combination with microfluidics. We also proposed a novel design for the AWG which appears to be advantageous especially when high diffraction orders are required. The design may lead to the development of AWGs with much higher resolutions than that of conventionally designed devices, due to its reduced dimensions and absence of systematic phase errors. We have also successfully demonstrated Raman spectroscopy from human tissue (in particular teeth) by using on-chip AWG spectrometers. With the promising results achieved in the scope of this thesis the feasibility is shown of advanced optical spectroscopic tools like Raman spectroscopy as mass-produced, low-cost systems. Once available these will open new applications in medicine, as well as food control and production, environmental monitoring and other fields.

The most important requirement for detecting a Raman signal is to have low losses in the propagation path of the light. This translates into choosing materials that exhibit low absorption of the Raman wavelengths and low scattering. Another condition is that the material must not present fluorescence in wavelength bands that overlap with the Raman signals to be measured. In our work we chose SiON. The high transparency of the material in the wavelength region of the Raman signals and the low level of luminescence were the key to our successful measurements. The material properties, the waveguide fabrication steps, as well as waveguide design have been discussed in Chapter 2.

In Chapter 3 we have discussed a semi-analytical model for describing waveguide probes. The model is valid for single-mode as well as multi-mode waveguides with arbitrary cross-sections. The most important result obtained with that model is that, in the case of thin samples $(\leq 100 \mu \mathrm{m})$, integrated probes present higher efficiencies and resolution compared to large-core multi-mode fiber probes. In addition, their performance exceeds that of small-core multi-mode and singlemode fiber probes for all sample thicknesses. These results encouraged us to find even better ways of collecting light with integrated optical devices. 
An innovative method has been introduced in Chapter 4 for simultaneously focusing laser light into the sample and confocally collecting the backscattered light without the need for external optics. This method is based on the use of a well known device in integrated optics: the AWG. We demonstrated that this method provides higher collection efficiency than can be achieved using a channel waveguide, and we gave a proof of principle showing that the device can be used as a microscope for imaging at multiple wavelengths.

In Chapter 5 we have discussed the designs of Raman spectrometers for the detection of the skin's natural moisturizing factor and early dental caries. For the first application we propose a novel layout for the AWG used for wavelength separation. The proposed layout is based on the use of identical bends across the entire grating, leading to a complete cancellation of systematic phase errors which are intrinsically present in conventional designs. We also show how the novel geometry can be used in the design of AWGs of any diffraction order and how, in case of high orders, it occupies a smaller area compared to the conventional horseshoe layout.

Finally, in Chapter 6 we have shown our experimental results on the detection of early dental caries using an AWG spectrometer. The results not only show that it is feasible to obtain Raman signals of the prominent hydroxyapatite peak at $\sim 959 \mathrm{~cm}^{-1}$ for both polarizations, but most importantly that the depolarization ratios measured with our device are in excellent agreement with the values found in the literature, as measured with conventional spectrometers.

\subsection{Future directions}

Our latest results have demonstrated the feasibility of on-chip Raman spectroscopy. These are certainly a motivation for continuing work in this field, as several challenges remain on the road towards realizing a fully functional, reliable and userfriendly Raman spectrometer on a chip. The main issues are with integrating laser suppression filters, laser line filters and polarization splitters on a single chip together with the wavelength selective devices.

An important step to take in future research is to improve the measurement setups used for characterizing the AWGs. In the currently used setups each output channel of the AWG must be measured separately, often leading to inconsistent data, since the coupling conditions cannot be maintained the same for each channel. In addition under the current conditions it may take more than 10 hours to measure 100-200 output channels. It would be of great importance to have a setup were the transmission spectra of all the output channels are measured simultaneously, for example by using an external grating and a CCD camera. This would give a reliable and fast (a matter of seconds) characterization of the AWGs. 
One of the problems that we encountered when designing the wavelength separation devices was the impossibility to achieve both a large free spectral range and a high resolution without increasing the device dimensions to unacceptably large values. In order to achieve these conditions, in future research one might consider using cascaded interconnections of AWGs. A more interesting approach would be to combine the AWG with tunable filters placed on the output channels. For example, grating based spectrometers, such as the AWG can be used to achieve large FSR with low resolution, while tunable narrow band-width filters placed at each output would enable one to obtain higher resolution at the expense of longer integration times. To keep the required acquisition time small, all the output filters should be scanned in parallel. Spectrometers having a large FSR and high resolution would be of general purpose and therefore, not only limited to one application, as the spectrometers presented in this work. This would definitely increase their market potential. 


\section{Appendix A}

\section{Design of identical-bend AWG}

I

$\mathrm{n}$ this appendix we describe the design of a generic AWG according to the geometry proposed in Section 5.2. The procedure does not lead to a single unique design, as it involves a number of arbitrary choices, some of which are restricted by the available waveguide fabrication technology. Other restrictions arise from topological feasibility requirements: waveguides should be laid out in such a way that they are everywhere sufficiently separated from each other and do not intersect with each other. We introduce the design equations, which will need to be solved iteratively.

The design starts from the initial specifications (central wavelength, wavelength resolution, FSR, waveguide geometry, etc.), from which a number of parameters is determined by use of the AWG design equations [Smi96], such as the length $R$ of the FPR, the arrayed-waveguide spacing $d$ at the FPR, the order $m$ of the AWG, the number of arrayed waveguides $N$, the length difference $\Delta L$, and the tilt $\Delta \alpha$ between adjacent waveguides. The minimum bending radius $r_{\min }$ that leads to acceptable bend losses is determined once the geometry of the waveguides is known. At this point the bend of type 2 can be designed, while the design of the bend of type 1 is related to the angle $\alpha$ and must be approached in an iterative way, as discussed later. Our design depends loosely on the bend choice, since the only bend parameter that enters the design is the chord of the bend $P$ (see Fig. 5.3) for the bend of type 1, and equivalently $\Delta P$ (not shown) for type 2 .

Our AWG layout is not anti-symmetric. The two halves of the AWG are different and need to be designed in separate steps. We commence with designing the left half:

1. We arbitrarily choose initial values for the constant $a$, the waveguide separation $s$ on the line $\overline{\mathrm{AB}}$, the angle $\alpha$, and the lengths of the straight sections $l_{p 1}$ and $l_{q 1}$ of the first waveguide (our reference waveguide).

2. Once $\alpha$ is determined, we choose the bend of type 1 and, therefore, the value of $P$.

3. From simple geometrical relations we find $D$ and $H$ (see Fig. 5.3).

4. For each waveguide of the left half $(i=2,3, \ldots, N)$, we determine the lengths of the three straight sections $l_{p i}, l_{q i}$, and $l_{r i}$.

Step 4 is performed analytically by solving a system of three equations, which is found in the following way. The $i^{\text {th }}$ waveguide has one bend of type 1 and $i-1$ bends of type 2 (dotted line in the figure) of length $l_{\Delta \alpha}$. The lengths of the waveguides belonging to the left half can be expressed as: 


$$
\begin{aligned}
& l_{1}=R+l_{p 1}+l_{\alpha}+l_{q 1} \\
& l_{2}=R+l_{p 2}+l_{\alpha}+l_{q 2}+l_{\Delta \alpha}+l_{r 2} \\
& \ldots \\
& l_{i}=R+l_{p i}+l_{\alpha}+l_{q i}+(i-1) l_{\Delta \alpha}+l_{r i}
\end{aligned}
$$

Recalling that the length difference between two adjacent waveguides on the left half is given by $a+\Delta L / 2$, we find a first equation by expressing the length difference between the $i^{\text {th }}$ waveguide and the reference waveguide as $(i-1)(a+$ $\Delta L / 2)$ :

$$
l_{p i}+l_{q i}+l_{r i}=(i-1)\left(a+\frac{\Delta L}{2}\right)+l_{p 1}+l_{q 1}-(i-1) l_{\Delta \alpha} .
$$

Two other equations can be derived from the requirements that the connection points of the waveguides are all on the line $\overline{\mathrm{AB}}$ and that these end points should be equidistant with a given spacing $s$. These equations read:

$$
\begin{aligned}
& \left(R+l_{p i}\right) \cos (\alpha+(i-1) \Delta \alpha)+P \cos (\alpha / 2+(i-1) \Delta \alpha)+. . \\
& \quad+l_{q i} \cos ((i-1) \Delta \alpha)+\sum_{j=1}^{i-1} \Delta P \cos ((i-1-j / 2) \Delta \alpha)+l_{r i}=D, \\
& \left(R+l_{p i}\right) \sin (\alpha+(i-1) \Delta \alpha)+P \sin (\alpha / 2+(i-1) \Delta \alpha)+. . \\
& \quad+l_{q i} \sin ((i-1) \Delta \alpha)+\sum_{j=1}^{i-1} \Delta P \sin ((i-1-j / 2) \Delta \alpha)=H+(i-1) s=H_{i}
\end{aligned}
$$

For each variation of $\alpha$ the steps 2, 3, and 4 need to be performed. By plotting the solutions of Eqs. (A.2)-(A.4) as a function of $\alpha$, we find the range of angles $\alpha$ for which all three lengths are non-negative. The angle $\alpha$ must be between 0 and $\pi / 2$ (an initial choice could be $\pi / 4$; the optimum value of $\alpha$ leads to the smallest footprint of the left half). If no value of $\alpha$ exists for which this condition is satisfied, the initial parameters $a, s, l_{p 1}$, and $l_{q 1}$ have to be adjusted. The procedure requires solving $N$ linear systems of three equations for three unknowns. The separation $s$ between the arrayed waveguides on line $\mathbf{A B}$ must guarantee that there is negligible coupling between the arrayed waveguides at the point where adjacent waveguides are closest to each other. Since the arrayed waveguides are tapered as they approach the FPR region, $l_{p 1}$ should be 2 or 3 times the taper length. A large value ( 2 or 3 times $\left.l_{p 1}\right)$ must be chosen for $l_{q 1}$.

For the design of the right half, see Fig. 5.3(b), of the AWG a similar procedure has to be applied:

1. To determine the lengths of the straight sections $l_{p N}^{\prime}$ and $l_{q N}^{\prime}$ of the topmost waveguide (the reference waveguide with number $N$ ), we impose two conditions -arbitrary to a degree- intended to make this second half of the AWG of similar size as the first half. The first condition sets the length of this waveguide, 


$$
l_{N}^{\prime}=R+l_{p N}^{\prime}+l_{\alpha}+l_{q N}^{\prime}=l_{1}+(N-1) \Delta L / 2,
$$

while the second condition is that $H^{\prime}=H$, which yields

$$
R \sin (\alpha)+l_{p N}^{\prime} \sin (\alpha)+P \sin (\alpha / 2)=H
$$

It is straightforward to solve this set of equations, as $R, l_{1}, l_{\alpha}, N, \Delta L, \alpha, P$, and $H$ have been determined before.

2. Subsequently, we find the value of $D^{\prime}$ (which is different from $D$ ) and then proceed, as before, by determining for each waveguide of the right half ( $i=$ $N-1, N-2, \ldots, 1)$ the lengths of the three straight sections $l_{p i}^{\prime}, l_{q i}^{\prime}$, and $l_{r i}^{\prime}$.

Our choice of imposing similar footprints makes it more likely that, with a certain choice of initial parameters, meaningful solutions are found for both halves of the AWG, thereby reducing the iteration steps necessary to design the geometry. 


\section{Appendix B}

\section{Design of laser suppression filters}

I

n order to suppress the reflected laser light and Rayleigh backscattered laser light which have several orders of magnitude higher power than the Raman signal, we need a filter that presents a strong suppression $(60-70 \mathrm{~dB})$ at the laser wavelength and a flat passband (with low losses) in the wavelength region of the Raman signal. In case of the NMF application this filter must present a large free spectral range (FSR > $115 \mathrm{~nm}$ ). In this case, in fact, the laser wavelength is 785 $\mathrm{nm}$, while the highest wavelength of the Raman signal to be detected is $900 \mathrm{~nm}$. An integrated optical filter consisting of a cascade of MZIs, a so-called lattice filter, can be designed to have a large FSR. One MZI element is schematically shown in Fig. B.1, and is constituted by the concatenation of three blocks, a first directional coupler with power coupling ratio $\kappa_{1}$, a delay line, and a second directional coupler with power coupling ratio $\kappa_{2}$. We indicate the field amplitudes at the input ports with $E_{i 1}$ and $E_{i 2}$, and at the output ports with $E_{o 1}$ and $E_{o 2}$.

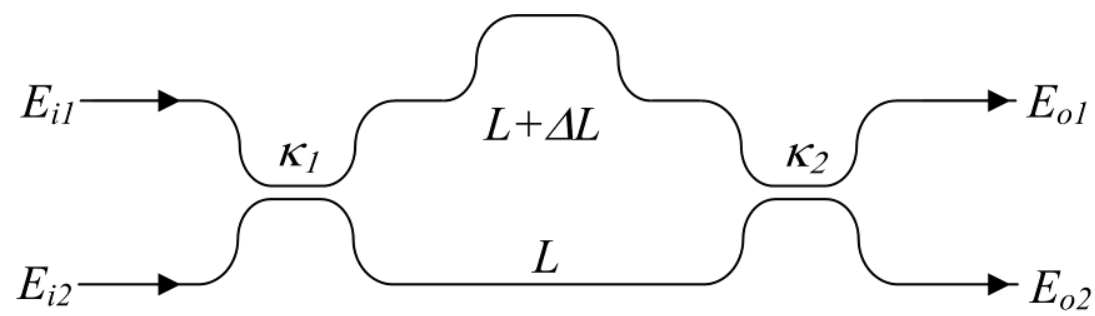

Fig. B.1 - Schematic diagram of a Mach-Zehnder interferometer (MZI).

The filter can be described by the transfer-matrix formulation [Mad99] as:

$$
\left[\begin{array}{l}
E_{o 1} \\
E_{o 2}
\end{array}\right]=\mathbf{H}\left[\begin{array}{l}
E_{i 1} \\
E_{i 2}
\end{array}\right],
$$

where $\mathbf{H}$ is the transfer matrix of the MZI, which is given by the product of the individual matrices of the constituting blocks in reverse order:

$$
\mathbf{H}=\mathbf{H}_{d c 2} \mathbf{H}_{d} \mathbf{H}_{d c 1} .
$$

In (5.2) $\mathbf{H}_{d c 1}$ and $\mathbf{H}_{d c 2}$ are the transfer matrices of the couplers, and $\mathbf{H}_{d}$ that of the delay line; they are given by:

$$
\mathbf{H}_{d c n}=\left[\begin{array}{cc}
\sqrt{1-\kappa_{\mathrm{n}}} & -j \sqrt{\kappa_{\mathrm{n}}} \\
-j \sqrt{\kappa_{\mathrm{n}}} & \sqrt{1-\kappa_{\mathrm{n}}}
\end{array}\right], \quad \mathrm{n}=1,2
$$




$$
\mathbf{H}_{d}=\gamma\left[\begin{array}{cc}
\gamma_{\Delta L} e^{-j k_{0} N_{e f f} \Delta L} & 0 \\
0 & 1
\end{array}\right]
$$

The factors $\gamma=\exp (-j \alpha L)$ and $\gamma_{\Delta L}=\exp (-j \alpha \Delta L)$ account for the losses in the delay line along the common path and the differential path, respectively. In the previous expressions $\alpha$ is the attenuation coefficient, $k_{0}$ is the propagation constant in vacuum given by $2 \pi / \lambda$, while $L$ and $\Delta L$ are the lengths of the common path and of the differential path (see Fig. B.1).

In Fig B.1 (a-b) we show the simulation results for two different single-MZI filters: the first MZI (see Fig B.2 (a)) is designed for using the through-port output (bar response), while the second (see Fig B.2 (b)) is designed for using the crossport output (cross response). Both MZIs are designed to present a stopband centered at $\lambda_{0}=785 \mathrm{~nm}$, by choosing a differential path-length of $\Delta L=m \lambda_{0} / N_{\text {eff }}$ for the through-port design, and $\Delta L=(2 m+1) \lambda_{0} / 2 N_{\text {eff }}$ for the cross-port design. Where $m$ is the order of the filter and is chosen ( $m=5$ in both cases) to have a high transmission between $830 \mathrm{~nm}$ and $900 \mathrm{~nm}$. The design was carried out assuming equal couplers with constant power coupling ratio $\left(\kappa_{1}=\kappa_{2}=0.5\right)$ over the spectral range between $750 \mathrm{~nm}$ and $930 \mathrm{~nm}$. We also analyzed the case where the power coupling ratios vary (in the same way) of \pm 0.1 with respect to the desired value of 0.5 . We observe (gray and dotted curves in Fig. B.2) that when using the bar response of the MZI for the filtering purpose, the rejection in the stopband is very sensitive to changes in the coupling coefficients. A change of \pm 0.1 in the power coupling ratios reduces the rejection from its ideal infinite value (ideally $0 \%$ transmission) to just about $15 \mathrm{~dB}$. We also observe that the same change in the coupling ratio does not affect the response from the cross port. This can be easily explained by considering the mathematical expressions of the two responses (field amplitude responses) where the propagation losses have been neglected:

$$
\begin{aligned}
& H_{b a r}=H_{11}=\sqrt{1-\kappa_{1}} \sqrt{1-\kappa_{2}} e^{-j k_{0} N_{e f f} \Delta L}-\sqrt{\kappa_{1}} \sqrt{\kappa_{2}} \\
& H_{\text {cross }}=H_{12}=-j\left(\sqrt{1-\kappa_{1}} \sqrt{\kappa_{2}} e^{-j k_{0} N_{e f f} \Delta L}+\sqrt{\kappa_{1}} \sqrt{1-\kappa_{2}}\right)
\end{aligned}
$$

From (5.4a) we observe that $H_{b a r}$ is zero when $\lambda=\lambda_{0}$, and $\kappa_{1}=1-\kappa_{2}$. While from (5.4b), $H_{\text {cross }}$ is zero when $\lambda=\lambda_{0}$, and $\kappa_{1}=\kappa_{2}$. From a fabrication point of view the latter condition is easier to achieve, since if the couplers are placed close to each other the technological variations will tend to affect them in the same way. The examined case does not take into account the wavelength dependency of the power coupling ratio, which is reported in Fig. B.3 for a directional coupler of $1000 \mu \mathrm{m}$ length, and $1.9 \mu \mathrm{m}$ gap having the same waveguide cross-section as described in Section 5.3.1. 


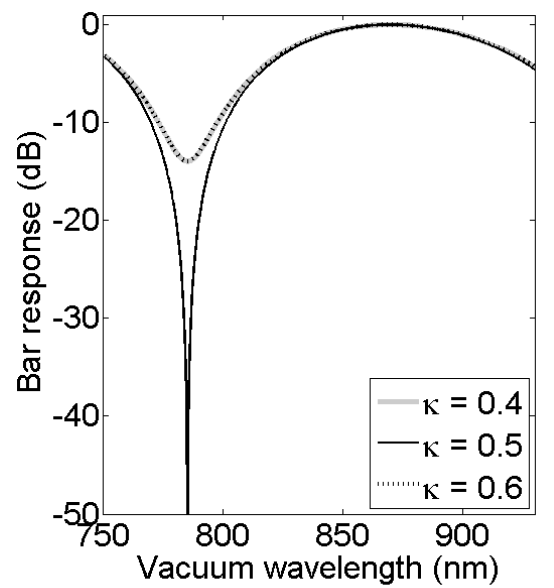

(a)

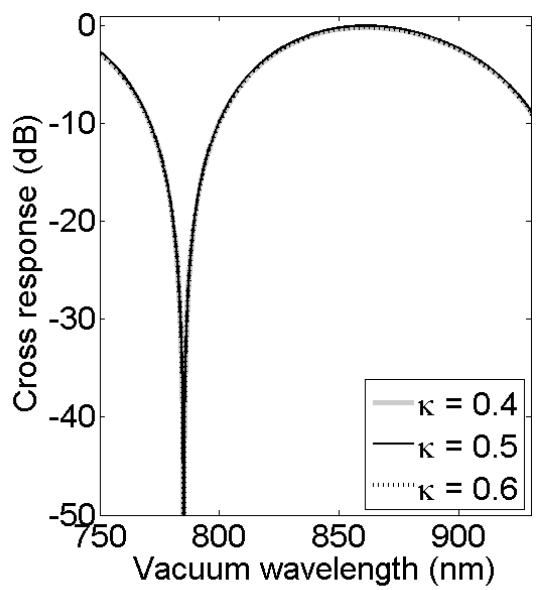

(b)

Fig. B.2 - The magnitude response of two different MZIs with equal wavelength independent couplers: (a) the through-port response of a MZI with stopband centered at $785 \mathrm{~nm}$ for three different values of the power coupling ratio; (b) the cross-port response of a MZI with stopband centered at $785 \mathrm{~nm}$ for three different values of the power coupling ratio.

As can be seen in Fig. B.3, the power coupling ratio varies significantly over the wavelength range $750 \mathrm{~nm}-930 \mathrm{~nm}$, and this variation should be accounted for in the simulations.

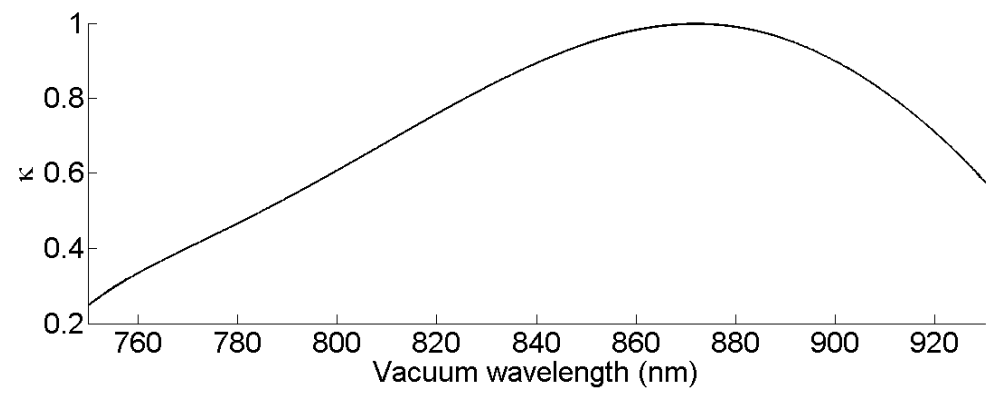

Fig. B.3 - Simulated power coupling ratio over the wavelength range $750 \mathrm{~nm}-930 \mathrm{~nm}$ for a directional coupler with $1000 \mu \mathrm{m}$ length and $1.9 \mu \mathrm{m}$ gap. The waveguide cross-section was $2 \mu \mathrm{m} \times 0.5 \mu \mathrm{m}$ (see Section 5.3.1).

When doing so, we find that for the cross-response there is still no significant decrease of the rejection in the stopband (Fig. B.4(b)), however, there is an increase of the losses in the passband, accompanied by a deterioration of the passband shape. On the other hand, the passband shape in case of the through-response (Fig. B.4(a)) becomes flat, but a significant decrease of the rejection in the stopband can still be observed together with a slight shift of central wavelength of the stopband. This makes the MZI not an ideal solution for our problem. We need a stable filter, which presents a flat passband with low losses and a high rejection of the laser line even 
when fabrication tolerances and wavelength dependencies of the various components are taken into account.

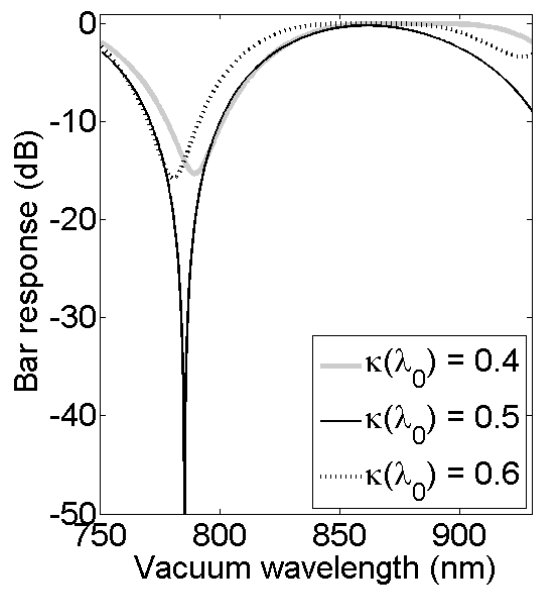

(a)

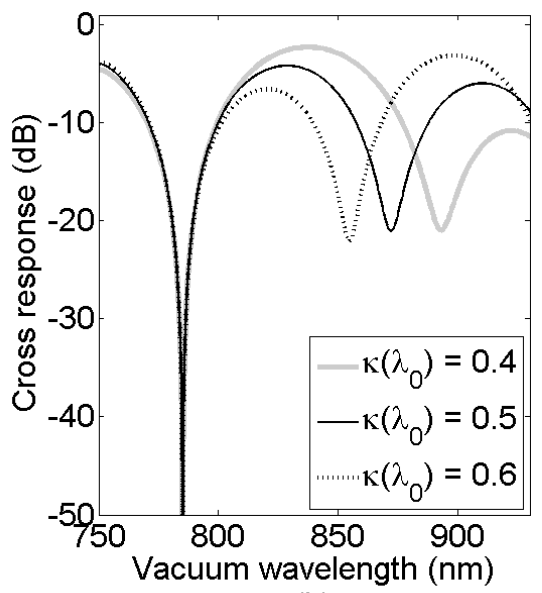

(b)

Fig. B.4 - The magnitude response of two different MZIs with equal wavelength dependent couplers: (a) the through-port response of a MZI with stopband centered at 785 $\mathrm{nm}$ for three different values of the power coupling ratio; (b) the cross-port response of a MZI with stopband centered at $785 \mathrm{~nm}$ for three different values of the power coupling ratio.

More stable filters with high rejection ratios can be obtained by cascading multiple MZIs [Mos84, Jin95, Mad99]. These filters, of which a schematic is shown in Fig. B.5, are also known as lattice filters.

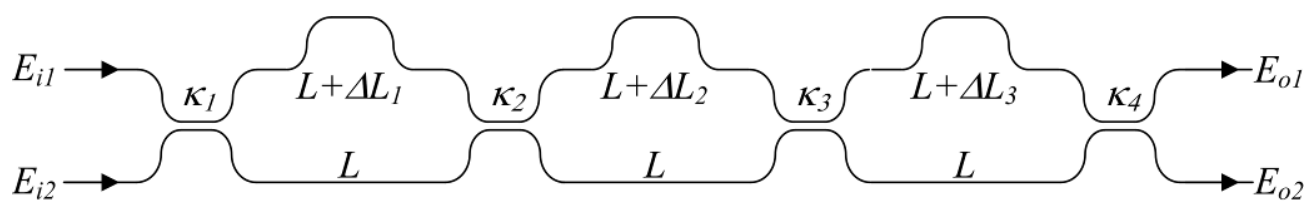

Fig. B.5 - Schematic diagram of a lattice filter.

In particular for the NMF application we choose a 3-stage filter as the one shown in Fig. B.5. Such a filter has seven parameters that can be arbitrarily chosen to optimize the design: the coupling coefficients of the four directional couplers, as well as the length of the three delay lines. Of course, also the position of the delay can be exchanged between upper and lower branch, but this can be accounted for as a negative value of the length difference $\Delta L$. In [Jin95] a synthesizing algorithm is presented to calculate the values of the different parameters in order to obtain the desired response. The method however, is intended for cases in which the frequency dependence in the coupling coefficients of the directional couplers is negligible. A different approach, which we applied in our design, is using genetic algorithms [Cus98]. The advantage of this approach is that the wavelength dependency of the 
couplers and different fabrication tolerances can be taken into account by using a lookup table to account for the dispersion of the couplers. Genetic algorithms have been extensively described in the literature where details of their implementation can be found [Gol88, Cus98, Dey10]. Here we will only describe the basic principle of this powerful method that enables us to find suitable parameters for the filter. In our design we require that all the couplers have the same power coupling ratios, which leads to a decrease of the number of unknown parameters from 7 to 4 (one coupling coefficient and the length of the three delay lines). In the design phase we reduce the number of free parameters further by fixing the power coupling ratio (at the laser wavelength). The free parameters are defined as genes, which form a vector that is called chromosome. In the first step of the genetic algorithm, an initial population of chromosomes is created randomly. For each chromosome of the initial population, the filter response function is computed and compared with the desired response. From the comparison a score (fitness parameter) is assigned to each chromosome. The evaluation is made in terms of flatness of the passband, maximum losses in the passband, and minimum rejection in the stopband and imposing that the maximum attenuation would be at $785 \mathrm{~nm} \pm 0.06 \mathrm{~nm}$ (the wavelength resolution used in the calculation). A constraint on the width of the stopband is not used. A group of chromosomes with higher fitness is selected from the initial population and used to create a new population through what is called offspring: the chromosome with maximum fitness is preserved while the others are subjected to random mutations and crossover. In the latter case a number of genes is exchanged between two chromosomes, where the number of exchanged genes and their positions are determined randomly. The process is repeated in a loop until a desired fitness value is met, or the number of maximum iterations is reached.

The laser suppression filter was designed such that the couplers had the same value of power coupling ratio of $\kappa(785 \mathrm{~nm})=\kappa_{0}=0.2$. The small value of the power coupling ratio corresponds to short couplers which can be simulated more accurately using 2D BPM, as shown in Chapter 1. 


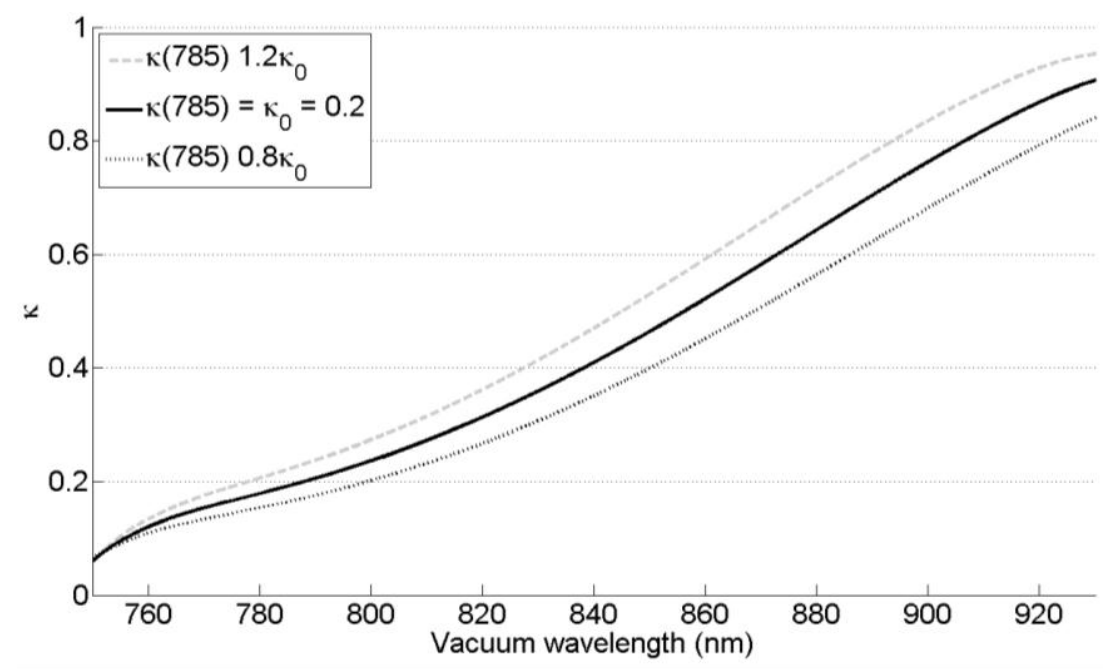

Fig. B.6 - Simulated power coupling ratio over the wavelength range $750 \mathrm{~nm}-930 \mathrm{~nm}$ for a directional coupler with $500 \mu \mathrm{m}$ length and $1.9 \mu \mathrm{m}$ gap (black line). For details on waveguide cross-section (see Section 5.3.1). We also show (dashed and dotted lines) the cases in which the coupling coefficient at $785 \mathrm{~nm}$ changed by approximately $\pm 20 \%$ with respect to the designed value of 0.2 (at $785 \mathrm{~nm}$ ).

With this choice, as can be seen from Fig. B.6, the coupling ratio $\kappa(\lambda)$ was almost a linear function of wavelength. To achieve value of $\kappa_{0}=0.2$ using the waveguide geometry described in the previous Section, a coupler length of $500 \mu \mathrm{m}$ and a gap of $1.9 \mu \mathrm{m}$ were chosen.

Using the genetic algorithm an optimum solution with high rejection at the laser wavelength was found. The rejection remained high, as shown in Fig. B.7, even when the power coupling ratio was varied (see Fig. B.6) by $\pm 20 \%$ at $785 \mathrm{~nm}$. The maximum rejection in the stopband fluctuated between $66 \mathrm{~dB}$ and $75 \mathrm{~dB}$, while the maximum losses in the passband were always below $1 \mathrm{~dB}$. The FWHM of the stopband was calculated with respect to the maximum rejection, and was in between $1 \mathrm{~nm}$ and $1.3 \mathrm{~nm}$. We observe that the stopband width is around 10 times larger than the line width of a typical laser user for Raman spectroscopy $(0.05 \mathrm{~nm}-0.1 \mathrm{~nm})$.

We could also verify that by introducing random phase errors up to $0.01 \pi$ in the three delay lines of the lattice filter the maximum losses in the passband would always stay below $1 \mathrm{~dB}$, while the stopband position would change even by $\pm 5 \mathrm{~nm}$, causing a reduction in the stopband rejection at $785 \mathrm{~nm}$ to $15 \mathrm{~dB}$ in the worst examined case. This leads to the conclusion that we need to introduce heaters on the delay lines in order to be able to fine tune the phase. However, our first tests are done without introducing the heaters in order to estimate the amplitudes of the phase errors that are to be expected, as well as the errors arising from the fabrication tolerances in the couplers. 


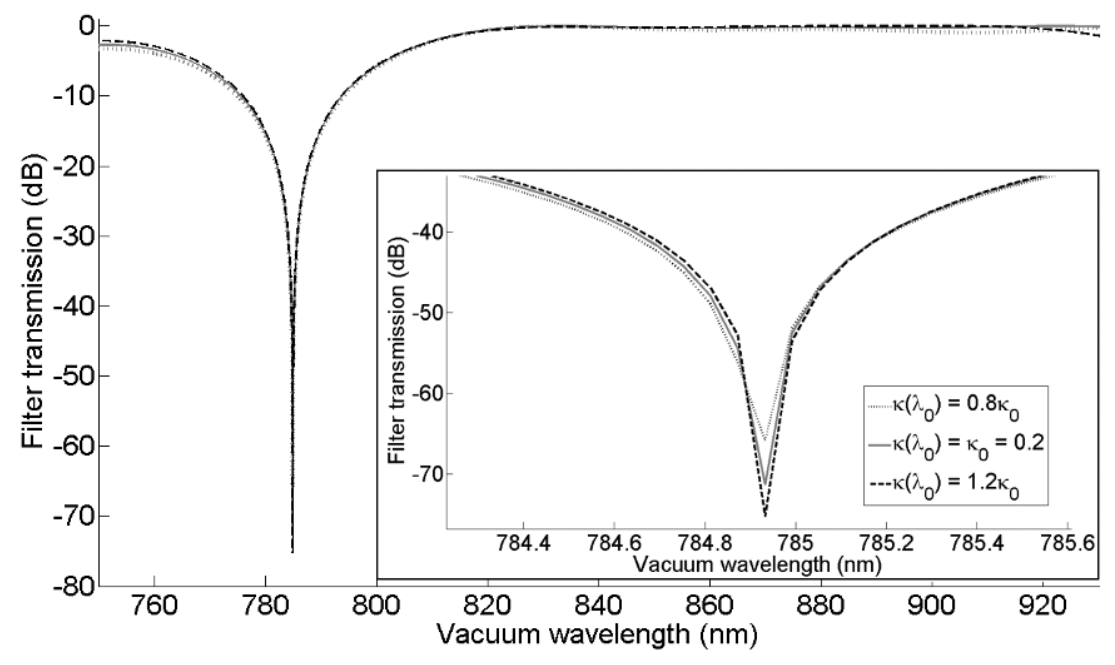

Fig. B.7 - Transmission of the laser-suppression filter in the wavelength region between $750 \mathrm{~nm}$ and $930 \mathrm{~nm}$ (dark gray line). We also show (dashed and dotted lines) the cases in which the coupling coefficient at $785 \mathrm{~nm}$ changed by approximately $\pm 20 \%$ with respect to the designed value of 0.2 (at $785 \mathrm{~nm}$ ).

The first fabrication attempt was not successful. The device presented a substantial shift $(11 \mathrm{~nm})$ in the stopband position, and a much lower rejection value than expected. Trying to fit the measured curve with our simulations we couldn't achieve a perfect fit, probably due to errors in the modeling of the couplers. The best obtained fit is show in Fig. B.8, from which we deduced that the degraded response shape and reduced rejection are attributable to unequal and increased power coupling ratios of the directional couplers, which were estimated (at $785 \mathrm{~nm}$ ) to be $\kappa_{1}=0.44, \kappa_{2}=0.42, \kappa_{3}=0.4$, and $\kappa_{4}=0.4$. The shift in the central wavelength of the stopband was, on the other hand, attributed to phase shifts of $-0.3 \pi,-0.1 \pi$ and $0.08 \pi$ present in the delay lines number 1,2 and 3, respectively. The measured response and the simulated responses with and without accounting for the fabrication non-uniformities are shown in Fig. B.8. A confirmation of the increased coupling is also obtained when comparing the measured transmission curves of the AWG (on the same wafer) in Fig. 5.10 with the simulated ones in Fig. 5.12. 


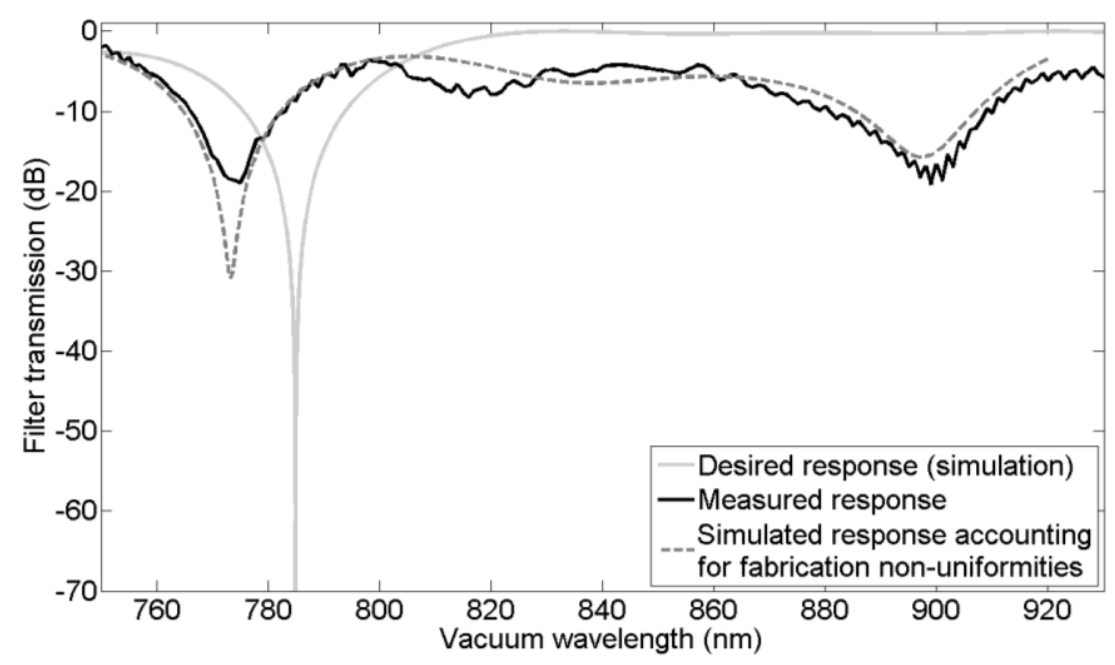

Fig. B.8 - Measured transmission of the laser-suppression filter (black line) compared with the desired response (gray line) and the simulated response accounting for fabrication non-uniformities (dashed gray line).

The filter was redesigned to take into account the errors introduced by the fabrication process. To compensate for the optical path deviations in the delay lines, heaters were introduced in each MZI arm. While to compensate for the increased coupling we opted for a simple solution of reducing the length of the couplers. Another option could have been the use of tunable couplers as described in [Roe02], where the performance of directional couplers fabricated in SiON is discussed. In that particular case a decrease in the coupling strength was observed (the measured power coupling ratio was 0.3 , while the designed value was 0.5 ), and tunable couplers were used to compensate for the deviation. The option of introducing tunable couplers leads to an increase in the device dimensions, since each coupler must be replaced by an MZI with two $3 \mathrm{~dB}$ couplers. 


\section{Appendix C}

\section{Design of an AWG based polarization splitter}

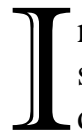

$\mathrm{n}$ order to separate the two orthogonally polarized components of the input signal and process them separately a polarization splitter is needed. Many different designs of integrated optical polarization splitters based on different principles and technologies have been proposed in the literature [Lia05, Sim03, Ye07, Shi10]. Here we propose a polarization splitter based on the arrayed waveguide grating which is, in general, a polarization dependent device [Spi96].

The polarization splitter must be designed to separate the TE and TM polarizations in a limited wavelength range of $22 \mathrm{~nm}$ (from $890 \mathrm{~nm}$ to $912 \mathrm{~nm}$ ), which coincides with the wavelength region of interest for the application. To achieve polarization splitting over this relatively large spectral range requires a device which presents high birefringence and low wavelength dependency. Furthermore, since the polarization splitter is on the same chip with other devices (laser suppression filters, the focusing and collection AWGs and the wavelength selection AWGs), it shares with these the optical properties of the guiding medium such as layer thickness and refractive index of core and cladding, making the design of the device even more challenging since the only parameter left that can be changed is the waveguide width. In our particular case, for the design of the devices for the dental application, we chose a layer thickness of $520 \mathrm{~nm}$. This choice was aimed at achieving single-mode waveguides (in the wavelength region of interest) with low birefringence. In fact, using low birefringent waveguides makes the design of the other devices easier, since for example the laser suppression filters for the TE and TM polarizations would not differ significantly, and neither would the designs of the wavelength selection AWGs (see Fig. 5.13).

The proposed polarization splitter design makes use of an identical-bend AWG layout with $N$ arrayed waveguides where both halves (see Figs. 5.3(a) and 5.3(b)) are designed imposing a diffraction order $m=0$. In this way, when connecting the left and right halves of the AWG, all the $N$ waveguides would have the same length. However, in order to introduce a difference in the optical paths of the two polarization components (TE and TM) we interpose an intermediate section of $N$ parallel straight waveguides of equal length $L$ (see Fig. C.1). Each of these waveguides is composed of three segments. The central segment has a smaller width $w_{2}$ and a much higher birefringence compared to the outer segments which have the same width $w_{1}$ as the waveguides of the left and right halves. The three segments are interconnected by the use of tapers having equal length $l_{\mathrm{t}}$ that do not contribute to the optical path length difference between the adjacent waveguides. It is important to observe that the diffraction order of the AWG is no longer $m=0$, but since we 
have introduced an intermediate section, it depends on the optical path length differences between the adjacent waveguides in this section. In the following we will indicate the diffraction order with $m^{\prime}$ to distinguish it from $m$.

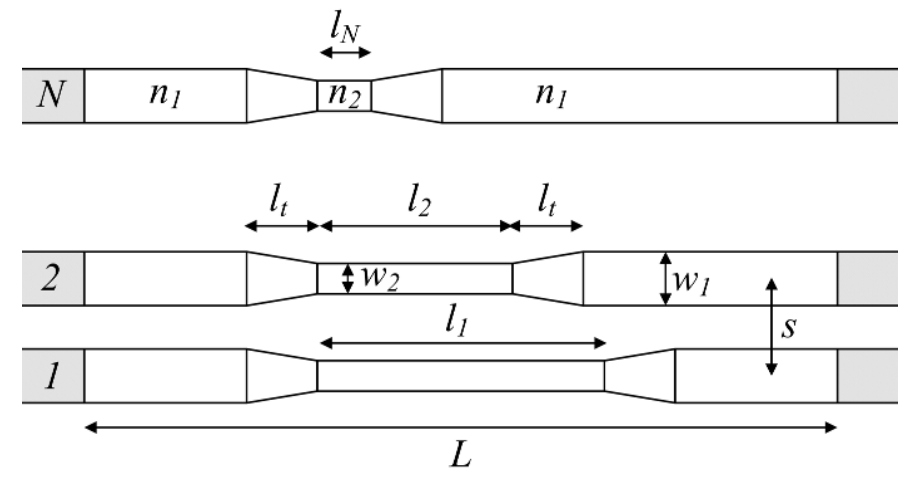

Fig. C.1 - Intermediate section of the AWG in which changes in the waveguide width are applied to introduce a difference in optical path between adjacent waveguides.

In general, when designing an AWG we first choose the central wavelength $\lambda_{\mathrm{c}}$ and then the optical path length difference between the adjacent waveguides, which is a multiple $m^{\prime}$ of the central wavelength. This translates into a physical length difference between adjacent waveguides given by $\Delta L=m^{\prime} \lambda_{\mathrm{d}} / n_{\text {eff }}$, where $n_{\text {eff }}$ is the effective index of the arrayed waveguides at the central wavelength.

In this case we also start by choosing a central wavelength $\lambda_{\mathrm{c}}=901 \mathrm{~nm}$ which false in the center of the spectral region of interest $890-912 \mathrm{~nm}$. However, this time all the waveguides of the AWG have the same physical length, and the optical path length difference between the adjacent waveguides depends on the lengths $\left(l_{1}, l_{2} \ldots\right.$ $l_{\mathrm{N}}$ ) of the central segments with smaller width shown in Fig. C.1. The length difference $l_{i}-l_{i-1}$ between these segments in two adjacent waveguides is chosen so that the optical path difference (for the TE polarization) between the waveguides is an integer multiple $m^{\prime}$ (we choose $m^{\prime}=1$ ) of the central wavelength $\lambda_{\mathrm{c}}$ :

$$
\left(L-l_{i}\right) n_{1}\left(\lambda_{c}, T E\right)+l_{i} n_{2}\left(\lambda_{c}, T E\right)-\left(L-l_{i-1}\right) n_{1}\left(\lambda_{c}, T E\right)-l_{i-1} n_{2}\left(\lambda_{c}, T E\right)=m^{\prime} \lambda_{c}
$$

In the previous equation we have indicated with $n_{1}\left(\lambda_{c}, T E\right)$ and $n_{2}\left(\lambda_{c}, T E\right)$ the effective indices (at the central wavelength $\lambda_{c}$ for the TE polarization) of the waveguide segments with width $w_{1}$, and $w_{2}$, respectively. From (C.1) we can easily determine the length difference:

$$
l_{i}-l_{i-1}=-\frac{m^{\prime} \lambda_{c}}{n_{1}\left(\lambda_{c}, T E\right)-n_{2}\left(\lambda_{c}, T E\right)} .
$$


The next step in the design of the AWG is to fix the wavelength spacing between the output channels (for the TE polarization). In order to collect all the wavelengths of the TE polarized Raman signal in the central output channel the wavelength spacing between the output channels must be $\Delta \lambda(T E)>22 \mathrm{~nm}$. We will fix the exact value at a later stage. In the following we make the assumption that the wavelength spacing between the output channels of the AWG is the same for the TE and the TM polarizations, $\Delta \lambda=\Delta \lambda(T M)=\Delta \lambda(T E)$.

At this point, in order to obtain the splitting of the two orthogonal polarizations into two adjacent output channels of the AWG, we must impose that the optical path length difference between two adjacent arrayed waveguides for the TM polarization and for the wavelength $\lambda_{c}$ is an integer multiple $m^{\prime}$ of the vacuum wavelength $\left(\lambda_{c}\right.$ $\Delta \lambda$ ). In this way TM polarized light at the wavelength $\lambda_{c}$ will fall into the output channel centered at wavelength $\lambda_{c}-\Delta \lambda$. In mathematical terms this translates into the equation:

$$
\begin{aligned}
& \left(L-l_{i}\right) n_{1}\left(\lambda_{c}, T M\right)+l_{i} n_{2}\left(\lambda_{c}, T M\right)- \\
& -\left(L-l_{i-1}\right) n_{1}\left(\lambda_{c}, T M\right)-l_{i-1} n_{2}\left(\lambda_{c}, T M\right)=m^{\prime}\left(\lambda_{c}-\Delta \lambda\right)
\end{aligned}
$$

From this relation immediately follows:

$$
l_{i}-l_{i-1}=-\frac{m^{\prime}\left(\lambda_{c}-\Delta \lambda\right)}{n_{1}\left(\lambda_{c}, T M\right)-n_{2}\left(\lambda_{c}, T M\right)} .
$$

Relation (C.4) has been written for wavelength $\lambda_{c}$. We can write the same relation for wavelength $\lambda_{c}+\Delta \lambda$ as follows:

$$
l_{i}-l_{i-1}=-\frac{m^{\prime} \lambda_{c}}{n_{1}\left(\lambda_{c}+\Delta \lambda, T M\right)-n_{2}\left(\lambda_{c}+\Delta \lambda, T M\right)} .
$$

By comparing (C.5) and (C.2) we obtain:

$$
n_{1}\left(\lambda_{c}+\Delta \lambda, T M\right)-n_{2}\left(\lambda_{c}+\Delta \lambda, T M\right)=n_{1}\left(\lambda_{c}, T E\right)-n_{2}\left(\lambda_{c}, T E\right),
$$

The effective indices $n_{1}$ and $n_{2}$ for the TE and TM polarizations depend on the channel waveguide geometries. Therefore, in order to satisfy the condition (C.6) and obtain polarization splitting we must choose the correct waveguide cross-sections. The waveguide cross-section used in the arrayed waveguides is the same as the one previously described for the wavelength selection AWG, thus $w_{1}=2.2 \mu \mathrm{m}$. The only free parameters that we can tune are the width $w_{2}$ of the central segment, and the wavelength spacing $\Delta \lambda$ of the output channels. On the latter we have the constraint $\Delta \lambda>22 \mathrm{~nm}$, while for the waveguide width $w_{2}$ the limitation is given by the 
minimum dimensions that can be achieved in the lithographic process, in general $>1$ $\mu \mathrm{m}$.

Condition (C.6) can be written in a more compact form in terms of the effective index difference $\Delta n_{12}=n_{1}-n_{2}$ as:

$$
\Delta n_{12}\left(\lambda_{c}+\Delta \lambda, T M\right)=\Delta n_{12}\left(\lambda_{c}, T E\right)
$$

In order to choose the two aforementioned parameters, we plot (see Fig. C.2) the difference $\Delta n_{12}\left(\lambda_{\mathrm{c}}+\Delta \lambda, T M\right)-\Delta n_{12}\left(\lambda_{\mathrm{c}}, T E\right)$ as a function of $\Delta \lambda$ and $w_{2}$, and choose the a point where the difference is zero and the above conditions are satisfied. We choose $w_{2} \sim 1 \mu \mathrm{m}$, which gives $\Delta \lambda_{\mathrm{c}} \sim 72 \mathrm{~nm}$.

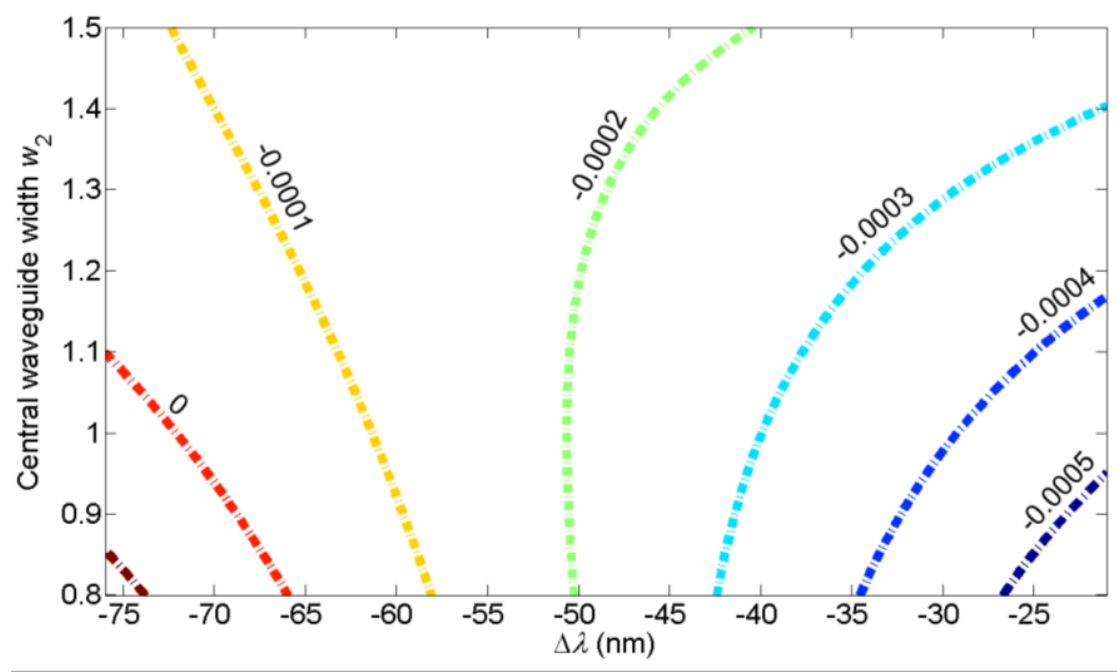

Fig. C. 2 - The difference $\Delta n_{12}\left(\lambda_{\mathrm{c}}+\Delta \lambda, T M\right)-\Delta n_{12}\left(\lambda_{\mathrm{c}}, T E\right)$ (color scale) as a function of $w_{2}$ and $\Delta \lambda$.

For the chosen values of $\Delta \lambda$ and $w_{2}$, the length difference between the central segments in adjacent arrayed waveguides is $l_{i-1}-l_{i} \cong 116.7 \mu \mathrm{m}$.

To complete the design of the AWG we must choose the length $R$ of the FPR and the spatial separation of the output channels of the AWG, as well as the separation $d$ between the arrayed waveguides at the intersection with the FPR (see Fig. 4.1 (a)). We choose of the separation between the arrayed waveguides and the output channels as a compromise between keeping the dimensions of the device small, and avoiding coupling between the waveguides. Both values are set to $7 \mu \mathrm{m}$. The length of the FPR can be easily determined once known the dispersion angle which can be calculated from Eq. (2) of [Smi96]. In our case we find $R \cong 0.9 \mathrm{~mm}$. Furthermore, as a compromise to limit the spillover losses, as well as the size of the device, we choose the number of arrayed waveguides $N$ equal to 41 . 
The polarization splitter was simulated using a $2 \mathrm{D}$ beam-propagation-method (BPM), and the simulated response in the wavelength range of interest for the application $(890 \mathrm{~nm}-912 \mathrm{~nm})$ is shown in Fig. C.3. As can be seen from the figure, the rejection between the two polarizations is variable, ranging from a maximum of $\sim 30 \mathrm{~dB}$, to a minimum of $\sim 12 \mathrm{~dB}$. We also observe that for the polarization splitter to work properly the input signal must span a limited spectral range preferably from $890 \mathrm{~nm}$ to $912 \mathrm{~nm}$. In order to ensure this condition is met the polarization splitter must be preceded (or followed) by a bandpass filter, which could be the collection AWG of Fig. 5.13, or alternatively a separate filter.

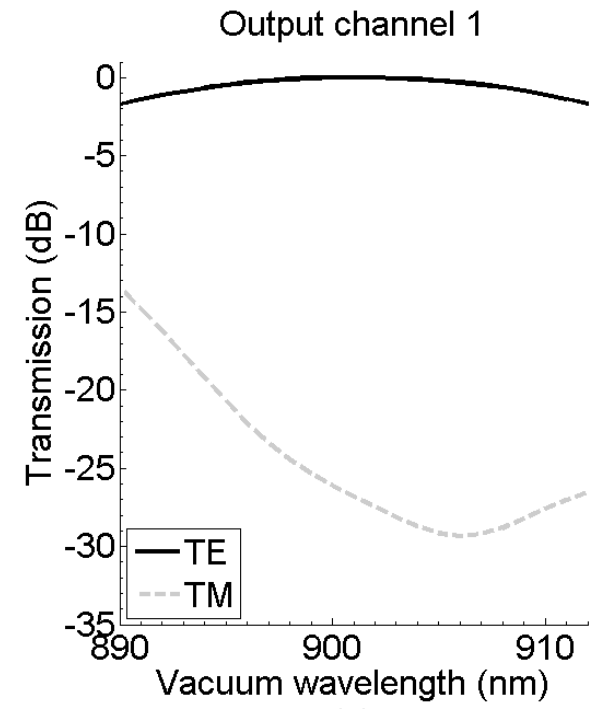

(a)

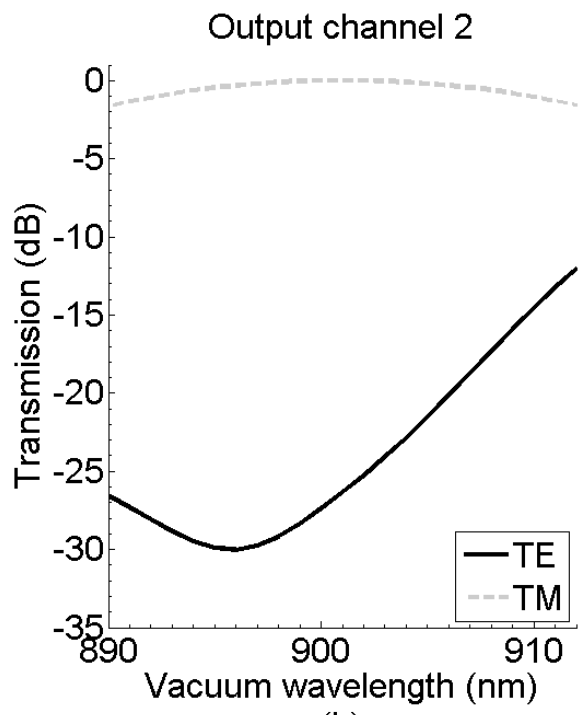

(b)

Fig. C.3 - Simulated response of the polarization splitter for the central output channel (number 1), and the adjacent channel (number 2).

The operation of the polarization splitter is based on the difference in effective indices between the arrayed waveguides having width $w_{1}$ and the thinner waveguide segments introduced in the intermediate section having width $w_{2}$. Therefore, the sensitivity of the polarization splitter to the changes in waveguide width due to fabrication tolerances is very high. If these changes are not controlled, for example introducing heaters to compensate for the changes in effective index due to fabrication inaccuracies the device may not work at all. We have, in fact, calculated that a variation in the width $w_{2}$ of only $\pm 0.1 \mu \mathrm{m}$ will cause the central wavelength $\lambda_{\mathrm{c}}$ of the AWG to shift by $\pm 100 \mathrm{~nm}$, while the same variation in $w_{1}$ will cause a shift of $\pm 40 \mathrm{~nm}$. 


\section{List of abbreviations}

$\begin{array}{ll}\text { AWG } & \text { Arrayed waveguide grating } \\ \text { BPM } & \text { Beam propagation method } \\ \text { BSA } & \text { Bovine serum albumin } \\ \text { CCD } & \text { Charge coupled device } \\ \text { ESF } & \text { Edge spread function } \\ \text { EIM } & \text { Effective index method } \\ \text { FPR } & \text { Free propagation region } \\ \text { FSR } & \text { Free spectral range } \\ \text { FWHM } & \text { Full-width-half-maximum } \\ \text { LPCVD } & \text { Low-pressure chemical vapour deposition } \\ \text { LSF } & \text { Line spread function } \\ \text { MTF } & \text { Modulation transfer function } \\ \text { MZI } & \text { Mach-Zehnder Interferometer } \\ \text { NIR } & \text { Near infra-red } \\ \text { NMF } & \text { Natural moisturizing factor } \\ \text { NA } & \text { Numerical aperture } \\ \text { OCT } & \text { Optical coherence tomography } \\ \text { PECVD } & \text { Plasma-enhanced chemical vapour deposition } \\ \text { PBS } & \text { Polarizing beam splitter } \\ \text { PM } & \text { Polarization maintaining } \\ \text { PRS } & \text { Polarized Raman spectroscopy } \\ \text { RIE } & \text { Reactive ion etching } \\ \text { SCA } & \text { Skin composition analyzer } \\ \text { SFPR } & \text { Sample-side free propagation region } \\ \text { TE } & \text { Transverse-electric } \\ \text { TEOS } & \text { Tetraethylorthosilicate } \\ \text { TM } & \text { Transverse-magnetic } \\ \text { UHNA } & \text { Ultra high numerical aperture } \\ \text { UV } & \text { Ultra violet } \\ & \end{array}$




\section{References}

[Ada93] Adar, R., C. H. Henry, C. Dragone, R. C. Kistler \& M. A. Milbrodt (1993) Broadband array multiplexers made with silica waveguides on silicon. Journal of Lightwave Technology, 11(2), 212-219.

[Alb95] Albers, H., L. T. N. Hilderink, E. Szilagyi, F. Paszti, P. V. Lambeck \& T. J. A. Popma (1995) Reduction of hydrogen induced losses in PECVD-SiO $\mathrm{N}_{\mathrm{y}}$ optical waveguides in the near infrared. Lasers and Electro-Optics Society Annual Meeting, 1995. 8th Annual Meeting Conference Proceedings, Volume 1., IEEE, pp. 88-89. San Francisco, CA, USA.

[Alb96] Albers, H., L. T. H. Hilderink, J. H. Berends, K. Wörhoff, N. F. v. Hulst \& P. V. Lambeck (1996) A waveguide material for integrated optical sensors: silicon oxynitride. National Sensor Conference, pp. 221-230. Delft, The Netherlands.

[Bad01] Bader, J. D., D. A. Shugars, G. Rozier, K. N. Lohr, A. J. Bonito, J. P. Nelson \& A. M. Jackman (2001) Diagnosis and Management of Dental Caries. AHRQ Publication No.01-E055. Agency for Healthcare Research and Quality.

[Ber05] Bernath, P. F. (2005) Spectra of atoms and molecules. Oxford University Press, New York, USA.

[Bin06a] Binzoni, T., T. S. Leung, A. H. Gandjbakhche, D. Rüfenacht \& D. T. Delpy (2006) Comment on 'The use of the Henyey-Greenstein phase function in Monte Carlo simulations in biomedical optics' Physics in medicine and biology, 51(22), L39.

[Bin06b] Binzoni, T., T. S. Leung, A. H. Gandjbakhche, D. Rüfenacht \& D. T. Delpy (2006) The use of the Henyey-Greenstein phase function in Monte Carlo simulations in biomedical optics Physics in medicine and biology, 51(17), N313.

[Boh98] Bohren, C. F. \& D. R. Huffman (1998) Absorption and scattering of light by small particles. Wiley, New York.

[Cal97] Calvo, M. L., A. Manzares, M. Chevalier \& V. Lakshminarayanan (1997) A formalism for analizing degraded edges using modified Heaviside functions. In V. Lakshminarayanan (ed.), Basic and Clinical Applications of Vision Science. Kluwer Academic, Dordrecht.

[Cal98] Calvo, M. L., A. Manzanares, M. Chevalier \& V. Lakshminarayanan (1998) Edge image quality assessment: a new formulation for degraded edge imaging. Image and Vision Computing, 16, 1003-1017.

[Cas98] Caspers, P. J., G. W. Lucassen, R. Wolthuis, H. A. Bruining \& G. J. Puppels (1998) In vitro and in vivo Raman spectroscopy of human skin. Biospectroscopy, 4, S31S39.

[Cas01] Caspers, P. J., G. W. Lucassen, E. A. Carter, H. A. Bruining \& G. J. Puppels (2001) In vivo confocal Raman microspectroscopy of the skin: noninvasive determination of molecular concentration profiles. The Journal of Investigative Dermatology, 116(3), 434-442.

[Cas03] Caspers, P. J., G. W. Lucassen \& G. J. Puppels (2003) Combined In vivo confocal Raman spectroscopy and confocal microscopy of human skin. Biophysical Journal, 85(1), 572-580.

[Chi07] Chicea, D. \& I. Turcu (2007) RWMCS - An alternative random walk Monte Carlo code to simulate light scattering in biological suspensions. Optik - International Journal for Light and Electron Optics, 118(5), 232-236.

[Cho10] Choo-Smith, L.-P. i., M. Hewko \& M. Sowa (2010) Towards early dental caries detection with OCT and polarized Raman spectroscopy. Optics Express, 2(Suppl 1), 043.

[Coo96] Cooney, T. F., H. T. Skinner \& S. M. Angel (1996) Comparative study of some 
fiber-optic remote Raman probe designs. Part I: Model for liquids and transparent solids. Applied Spectroscopy, 50(7), 836-848.

[Cro66] Cronemeyer, D. C. (1966) Optical absorption characteristics of pink ruby. Journal of the optical society of America, 56(12), 1703-1705.

[Cus98] Cusick, T. A., S. lezekiel \& R. E. Miles (1998) All-optical microwave filter design employing a genetic algorithm. Photonics Technology Letters, 10(8), 1156-1158.

[Dai05] Dainese, M. (2005) Plasma assited technology for Si-based photonic integrated circuits. Microelectronics and Information Technology. Royal Institute of Technology (KTH), Stockholm, Sweden.

[Den07] Denson, S. C., C. J. S. Pommier \& M. B. Denton (2007) The impact of array detectors on Raman spectroscopy. Journal of Chemical Education, 84(1), 67-74.

[Dey10] Dey, A. K., S. Saha, A. Saha \& S. Ghosh (2010) A method of genetic algorithm (GA) for FIR filter construction: design and development with newer approaches in neural network platform. International Journal of Advanced Computer Science and Applications, 1(6), 87-90.

[Dra89] Dragone, C. (1989) Efficient N x N star couplers using Fourier optics. Journal of Lightwave Technology, 7(3), 479-489.

[Dra91] Dragone, C. (1991) An N x N optical multiplexer using a planar arrangement of two star couplers. Photonics Technology Letters, 3(9), 812-815.

[Fen47] Fenske, M. R., W. G. Braun, R. V. Wiegand, D. Quiggle, R. H. McCormick \& D. H. Rank (1947) Raman Spectra of Hydrocarbons. Analytical Chemistry, 19(10), 700765.

[Fer03] Ferraro, J. R., K. Nakamoto \& C. W. Brown (2003) Preface to the Second Edition. Introductory Raman Spectroscopy (Second Edition). Academic Press, San Diego.

[Gar85] Garcia, M. V. \& M. I. Redondo (1985) Identification of the stable conformer of cyclohexane by vibrational spectroscopy. Journal of Chemical Education, 62(10), 887-889.

[Ger00] Germann, R., H. W. M. Salemink, R. Beyeler, G. L. Bona, F. Horst, I. Massarek \& B. J. Offrein (2000) Silicon Oxynitride Layers for Optical Waveguide Applications. Journal of the Electrochemical Society, 147(6), 2237-2241.

[Goh97] Goh, T., S. Suzuki \& A. Sugita (1997) Estimation of waveguide phase error in silica-based waveguides. J. of Lightw. Technol., 15(11), 2107-2113.

[Gol88] Goldberg, D. E. \& J. H. Holland (1988) Genetic Algorithms and Machine Learning. Machine Learning, 3(2), 95-99.

[Hal79] Hall, D. G., R. R. Rice \& J. D. Zino (1979) Simple Gaussian-beam model for GaAlAs double-heterostructure laser-diode-to-diffused-waveguide coupling calculations. Optics Letters, 4(9), 292-294.

[Han00] Hanlon, E. B., R. Manoharan, T.-W. Koo, K. E. Shafer, J. T. Motz, M. Fitzmaurice, J. R. Kramer, I. Itzkan, R. R. Dasari \& M. S. Feld (2000) Prospects for in vivo Raman spectroscopy. Physics in medicine and biology, 45(2), R1-R59.

[Hen41] Henyey, L. G. \& J. L. Greenstein (1941) Diffuse radiation in the Galaxy. The Astrophysical Journal, 93, 70-83.

[Her76] Hermann, R. \& J. Hertel (1976) Mode launching on a multimode slab-waveguide by a plane wave. Applied Physics A: Materials Science \& Processing, 9(4), 307-313.

[Hil00] Hill, W. \& V. Petrou (2000) Caries Detection by Diode Laser Raman Spectroscopy. Applied Spectroscopy, 54(6), 795-799.

[Hoc77] Hocker, G. B. \& W. K. Burns (1977) Mode dispersion in diffused channel waveguides by the effective index method. Applied Optics, 16(1), 113-118.

[Hol08] Holst, G. C. (2008) Testing and Evaluation of Infrared Imaging Systems. Spie Press Monograph.

[Hor04] Horowitz, A. M. (2004) A report on the NIH consensus development conference on diagnosis and management of dental caries throughout life. Journal of Dental Research, 83(Spec Iss C), C15-C17.

[Hul81] Hulst, H. C. V. d. (1981) Light scattering by small particles. Dover Publications, New York. 
[Hus01] Hussein, M. G., K. Wörhoff, C. G. H. Roeloffzen, L. T. H. Hilderink, R. M. de Ridder \& A. Driessen (2001) Characterization of thermally treated PECVD SiON layers. In IEEE (ed.), 6th Annual Symposium IEEE/LEOS Benelux Chapter, pp. 265-268. Brussels, Belgium.

[Hut82] Hutley, M. C. (1982) Diffraction gratings. New York.

[Ind83] Indebetouw, G. (1983) Some experiments in partially coherent imaging and modulation transfer function evaluation. Applied Physics B, 32(1), 21-24.

[Ish77] Ishimaru, A. (1977) Theory and application of wave propagation and scattering in random media. Proceedings of the IEEE, 65(7), 1030-1061.

[IsI93] IslamRaja, M. M., C. Chang, J. P. McVittie, M. A. Cappelli \& K. C. Saraswat (1993) Two precursor model for low-pressure chemical vapor deposition of silicon dioxide from tetraethylorthosilicate. Journal of Vacuum Science \& Technology B: Microelectronics and Nanometer Structures, 11(3), 720-726.

[Ism04] Ismail, A. I. (2004) Visual and Visuo-tactile detection of dental caries. Journal of Dental Research, 83(Spec Iss C), C56-C66.

[Jan04] Janz, S., A. Balakrishnan, S. Charbonneau, P. Cheben, M. Cloutier, A. Delâge, K. Dossou, L. Erickson, M. Gao, P. A. Krug, B. Lamontagne, M. Packirisamy, M. Pearson \& D.-X. Xu (2004) Planar waveguide Echelle gratings in silica-on-silicon. Photonics Technology Letters, 16(2), 503-505.

[Jin95] Jinguji, K. \& M. Kawachi (1995) Synthesis of coherent two-port lattice-form optical delay-line circuit. Journal of Lightwave Technology, 13(1), 73-82.

[Kaw01] Kawano, K. \& T. Kitoh (2001) Introduction to optical waveguide analysis. WileyInterscience, New York, USA.

[Ko05] Ko, A. C.-T., L.-P. i. Choo-Smith, M. Hewko, L. Leonardi, M. G. Sowa, C. C. S. Dong, P. Williams \& B. Cleghorn (2005) Ex vivo detection and characterization of early dental caries by optical coherence tomography and Raman spectroscopy. Journal of Biomedical Optics, 10(3), 031118.

[Ko06] Ko, A. C.-T., L.-P. i. Choo-Smith, M. Hewko, M. G. Sowa, C. C. S. Dong \& B. Cleghorn (2006) Detection of early dental caries using polarized Raman spectroscopy. Optics Express, 14(1), 203-215.

[Ko08] Ko, A. C., M. Hewko, M. G. Sowa, C. C. Dong, B. Cleghorn \& L.-P. i. Choo-Smith (2008) Early dental caries detection using a fibre-optic coupled polarizationresolved Raman spectroscopic system. Optics Express, 16(9), 6274-6284.

[Kon92] Kong Chong, C., C. Shen, Y. Fong, J. Zhu, F.-X. Yan, S. Brush, C. K. Mann \& T. J. Vickers (1992) Raman spectroscopy with a fiber-optic probe. Vibrational Spectroscopy, 3(1), 35-45.

[Koy83] Koyama, J., I. Horii, K. Kawasaki, Y. Nakayama, Y. Morikawa, T. Mitsui \& H. Kumagai (1983) Free amino acids of stratum corneum as a biochemical marker to evaluate dry skin. Journal of the Society of Cosmetic Chemists, 35(4), 183-195.

[Lee01] Lee, C. D., W. Chen, Q. Wang, Y.-J. Chen, W. T. Beard, D. Stone, R. F. Smith, R. Mincher \& I. R. Stewart (2001) The role of photomask resolution on the performance of arrayed-waveguide grating devices. J. of Lightw. Technol., 19(11), 1726-1733.

[Lew93] Lewis, E. N., P. J. Treado \& I. W. Levin (1993) A miniaturized, no-moving-parts Raman spectrometer. Journal of Applied Spectroscopy, 47(5), 539-543.

[Lew96] Lewis, I. R. \& P. R. Griffith (1996) Raman spectrometry with fiber-optic sampling. Applied Spectroscopy, 50(10), 12A-30A.

[Lia05] Liang, T. K. \& H. K. Tsang (2005) Integrated polarization beam splitter in high index contrast silicon-on-insulator waveguides. Photonics Technology Letters, 17(2), 393-395.

[Lon77] Long, D. A. (1977) Raman spectroscopy. McGraw-Hill, London, UK.

[Ma03] Ma, X., J. Q. Lu, R. S. Brock, K. M. Jacobs, P. Yang \& X.-H. Hu (2003) Determination of complex refractive index of polystyrene microspheres from 370 to $1610 \mathrm{~nm}$. Physics in medicine and biology, 48, 4165-4172.

[Mad99] Madsen, C. K. \& J. H. Zhao (1999) Optical filter design and analysis: a signal 
processing approach. Wiley-Interscience, New York.

[Mai61] Maiman, T. H., R. H. Hoskins, I. J. D'Haenens, C. K. Asawa \& V. Evtuhov (1961) Stimulated optical emission in fluorescent solids. II. Spectroscopy and stimulated emission in ruby. Physiscal Review, 123(4), 1151-1157.

[Mal65] Malitson, I. H. (1965) Interspecimen comparison of the refractive index of fused silica. J. Opt. Soc. Am., 55(10), 1205-1208.

[Man97] Manzanares, A., M. L. Calvo, M. Chevalier \& V. Lakshminarayanan (1997) Line spread function formulation proposed by W. H. Steel: a revision. Applied Optics, 36(19), 4362-4366.

[Mor99] Morales-Acevedo, A., J. Carrillo-López, M. Aceves, W. Calleja-Arriaga, E. DíazValdés, G. Contreras \& C. Mejía-García (1999) Optical properties, infrared spectroscopy and photoluminescence at low temperature of LPCVD silicon oxynitride thin films. Superficies y Vacio, 9, 56-58.

[Mos84] Moslehi, B., J. W. Goodman, M. Tur \& H. J. Shaw (1984) Fiber-optic lattice signal processing. Proceedings of the IEEE, 72(7), 909-930.

[Mun91] Munowitz, M. \& D. J. Vezzetti (1991) Numerical procedures for constructing equivalent slab waveguides. An alternative approach to effective-index theory. Journal of Lightwave Technology, 9(9), 1068-1073.

[Oli93] Olivier, P., S. Rioux \& D. Gagnon (1993) Mathematical modeling of the solid angle function, part II: transmission through refractive media. Optical Engineering, 32(9), 2266-2270.

[Pas05] Passos, D., J. C. Hebden, P. N. Pinto \& R. Guerra (2005) Tissue phantom for optical diagnostics based on a suspension of microspheres with a fractal size distribution. Journal of Biomedical Optics, 10(6), Article 064036.

[Pel99] Pelletier, M. J. (1999) Effects of temperature on cyclohexane Raman bands. Applied Spectroscopy, 53(9), 1087-1096.

[Pet91] Peters, D., K. Fischer \& J. Müller (1991) Integrated optics based on silicon oxynitride thin films deposited on silicon substrates for sensor applications. Sensors and Actuators A: Physical, 26(1-3), 425-431.

[Pla86] Plaza, P., N. Q. Dao, M. Jouan, H. Fevrier \& H. Saisse (1986) Simulation et optimisation des capteurs a fibres optiques adjacentes. Applied Optics, 25(19), 3448-3454.

[Pra89] Prahl, S. A., M. Keijzer, S. L. Jacques \& A. J. Welch (1989) A Monte Carlo model of light propagation in tissue. SPIE Proceedings of Dosimetry of Laser Radiation in Medicine and Biology. Press.

[Pri99] Price, K. J., L. E. McNeil, A. Suvkanov, E. A. Irene, P. J. MacFarlane \& M. E. Zvanut (1999) Characterization of the luminescence center in photo- and electroluminescent amorphous silicon oxynitride films. Journal of Applied Physics, 86(5), 2628-2637.

[Qui95] Quinten, M. \& J. Stier (1995) Absorption of scattered light in colloidal systems of aggregated particles. Colloid \& Polymer Science, 272(3), 233-241.

[Ram28 $\left.{ }^{a}\right]$ Raman, C. V. \& K. S. Krishnan (1928) A new type of secondary radiation. Nature, 121, 501-502.

[Ram28 $\left.{ }^{b}\right]$ Raman, C. V. (1928) A change of wave-length in light scattering. Nature, 121, 619619.

[Ram28 ${ }^{C}$ Raman, C. V. (1928) A new radiation. Indian Journal of Physics, 2, 388-398.

[Rid98] de Ridder, R. M., K. Wörhoff, A. Driessen, P. V. Lambeck \& H. Albers (1998) Silicon oxynitride planar waveguiding structures for application in optical communication. Journal of Selected Topics in Quantum Electronics, 4(6), 930-937.

[Roe02] Roeloffzen, C. (2002) Passband flattened binary-tree structured add-drop multiplexers using SiON waveguide technology. University of Twente, Enschede.

[Ru09] Le Ru, E. C. \& P. G. Etchegoin (2009) Raman spectroscopy and related optical techniques. Principles of Surface-Enhanced Raman Spectroscopy. Elsevier, Amsterdam.

[Sch84] Schwab, S. D. \& R. L. McCreery (1984) Versatile, efficient Raman sampling with 
fiber optics. Analytical Chemistry, 56(12), 2199-2204.

[Sch90] Schiebener, P. \& J. Straub (1990) Refractive index of water and steam as function of wavelength, temperature and density. Journal of Physical and Chemical Reference Data, 19(3), 677-717.

[Sen11] Sengo, G., H. A. G. M. Wolferen \& A. Driessen (2011) Optimized Deep UV Curing Process for Metal-Free Dry-Etching of Critical Integrated Optical Devices. Journal of the Electrochemical Society, 158(10), H1084--H1089.

[She08] Sheehan, R. N., S. Horne \& F. H. Peters (2008) The design of low-loss curved waveguides. Optical and Quantum Electronics, 40(Special Issue on Numerical Simulation of Optoelectronic Devices (2008)), 1211-1218.

[Shi10] Shi, Y. C. (2010) A compact polarization beam splitter based on a multimode photonic crystal waveguide with an internal photonic crystal section. Progress In Electromagnetics Research, 103, 393-401.

[Sim03] Simova, E. \& I. Golub (2003) Polarization splitter/combiner in high index contrast Bragg reflector waveguides. Optics Express, 11(25), 3425-3430.

[Sin02] Singh, R. (2002) C. V. Raman and the Discovery of the Raman Effect. Physics in Perspective, 4(4), 399-420.

[Smi81] Smith, R. C. \& K. S. Baker (1981) Optical properties of the clearest natural waters (200-800 nm). Applied Optics, 20(2), 177-184.

[Smi88] Smit, M. K. (1988) New focusing and dispersive planar component based on an optical phased array. Electronics Letters, 24(7), 385-386.

[Smi96] Smit, M. K. \& C. v. Dam (1996) PHASAR-Based WDM-Devices: Principles, Design and Applications. J. of Selected Topics in Quantum Electronics, 2(2), 236-250.

[Soa08] Soares, F. M., W. Jiang, N. K. Fontaine, S. W. Seo, J. H. Baek, R. G. Broeke, J. Cao, K. Okamoto, F. Olsson, S. Lourdudoss \& S. J. B. Yoo (2008) InP-Based Arrayed-Waveguide Grating with a Channel Spacing of $10 \mathrm{GHz}$. National Fiber Optic Engineers Conference. Optical Society of America, California.

[Som09] Sometani, M., R. Hasunuma, M. Ogino, H. Kuribayashi, Y. Sugahara \& K. Yamabe

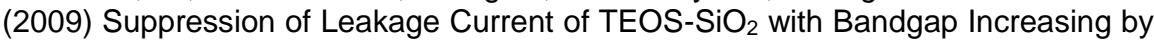
High Temperature Annealing. ECS Meeting Abstracts, 901(19), 801.

[Spi96] Spiekman, L. H., M. R. Amersfoort, A. H. d. Vreede, F. P. G. M. v. Ham, A. Kuntze, J. W. Pedersen, P. Demeester \& M. K. Smit (1996) Design and realization of polarization independent phased array wavelength demultiplexers using different array orders for TE and TM. Journal of Lightwave Technology, 14(06), 991-995.

[Syl10] Sylvestre, J.-P., C. C. Bouissou, R. H. Guy \& M. B. Delgado-Charro (2010) Extraction and quantification of amino acids in human stratum corneum in vivo. British Journal of Dermatology, 163(3), 458-465.

[Tak90] Takahashi, H., S. Suzuki, K. Kato \& I. Nishi (1990) Arrayed-waveguide grating for wavelength division multi/demultiplexer with nanometer resolution. Electronics Letters, 26(2), 87-88.

[Tak95] Takahashi, H., K. Oda, H. Toba \& Y. Inoue (1995) Transmission characteristics of arrayed waveguide $\mathrm{N} \times \mathrm{N}$ wavelength multiplexer. Journal of Lightwave Technology, 13(3), 447-455.

[Tak02] Takada, K., M. Abe, T. Shibata \& K. Okamoto (2002) 1-GHz-spaced 16-channel arrayed-waveguide grating for a wavelength reference standard in DWDM network systems. Journal of Lightwave Technology, 20(5), 850-853.

[Tan06] Tanaka, M. \& R. J. Young (2006) Review: Polarised Raman spectroscopy for the study of molecular orientation distributions in polymers. Journal of Materials Science, 41(3), 963-991.

[Tat84] Tatian, B. (1984) Fitting refractive-index data with the Sellmeier dispersion formula. Applied Optics, 23(24), 4477-4485.

[Utz03] Utzinger, U. \& R. R. Richards-Kortum (2003) Fiber optic probes for biomedical optical spectroscopy. Journal of Biomedical Optics, 8(1), 121-147.

[Wan95] Wang, L., S. L. Jacques \& L. Zheng (1995) MCML - Monte Carlo modeling of light transport in multi-layered tissues. Computer Methods and Programs in 
Biomedicine, 47, 131-146.

[Wan06] Wang, Q., G. Farrell \& T. Freir (2006) Effective index method for planar lightwave circuits containing directional couplers. Optics Communications, 259(1), 133-136.

[Wis80] Wiscombe, W. J. (1980) Improved Mie scattering algorithms. Applied Optics, 19(9), 1505-1509.

[Wit77] Witt, A. N. (1977) Multiple scattering in reflection nebulae. The Astrophysical Journal Supplement Series, 35, 1-6.

[Wör98] Wörhoff, K., R. M. de Ridder, P. V. Lambeck \& A. Driessen (1998) Silicon oxynitride in integrated optics. In IEEE (ed.), Lasers and Electro-Optics Society Annual Meeting, pp. 370-371. Orlando, USA.

[Wör99a] Wörhoff, K., A. Driessen, P. V. Lambeck, L. T. H. Hilderink, P. W. C. Linders \& T. J. A. Popma (1999) Plasma enhanced chemical vapor deposition silicon oxynitride optimized for application in integrated optics. Sensors and Actuators A: Physical, 74(1-3), 9-12.

[Wör99b] Wörhoff, K., P. V. Lambeck \& A. Driessen (1999) Design, tolerance analysis, and fabrication of silicon oxynitride based planar optical waveguides for communication devices. Journal of Lightwave Technology, 17(8), 1401-1407.

[Wör02] Wörhoff, K., L. T. H. Hilderink, A. Driessen \& P. V. Lambeck (2002) Silicon oxynitride: a versatile material for integrated optics application. Journal of the Electrochemical Society, 149, 85-91.

[Wör07] Wörhoff, K., C. G. H. Roeloffzen, R. M. d. Ridder, A. Driessen \& P. V. Lambeck (2007) Design and application of compact and highly tolerant polarization independent waveguides. Journal of Lightwave Technology, 25(5), 1276-1283.

[Wör08] Wörhoff, K., E. Klein, G. Hussein \& A. Driessen (2008) Silicon Oxynitride Based Photonics. 10th International Conference on Transparent Optical Networks, vol 3, pp. 266-269, Paper Th.B263.264. Athens, Greece.

[Wun92] Wunderlich, S., J. P. Schmidt \& J. Müller (1992) Integration of SION waveguides and photodiodes on silicon substrates. Applied Optics, 31(21), 4186-4189.

[Ye07] Ye, W. N., D.-X. Xu, S. Janz, P. Waldron, P. Cheben \& N. G. Tarr (2007) Passive broadband silicon-on-insulator polarization splitter. Optics Letters, 32(11), 14921494.

[Yin03a] Yin, X., J. Goudriaan, E. A. Lantinga, J. Vos \& H. J. Spiertz (2003) A flexible sigmoid function of determinate growth. Annals of Botany, 91(3), 361-371.

[Yin03b] Yin, X., J. Goudriaan, E. A. Lantinga, J. Vos \& H. J. Spiertz (2003) ERRATUM - A flexible sigmoid function of determinate growth. Annals of Botany, 91(6), 753.

[Zha10] Zhang, W., C. Hou, Y. Geng \& G. Yang (2010) Closely packed micro optical fiber arrays in laser scanning system. Optical and Quantum Electronics, 41(14), 981988.

[Zol06] Zolek, N. S., A. Liebert \& R. Maniewski (2006) Optimization of the Monte Carlo code for modeling of photon migration in tissue. Computer Methods and Programs in Biomedicine, 84(1), 50-57.

[Zol08] Zolek, N. S., S. Wojtkiewicz \& A. Liebert (2008) Correction of anisotropy coefficient in original Henyey Greenstein phase function for Monte Carlo simulations of light transport in tissue. Biocybernetics and Biomedical Engineering, 28(4), 59-73. 


\section{Samenvatting}

$\mathrm{R}$ amanspectroscopie is in opkomst als een krachtig gereedschap in vele toepassingen, zoals virologie, farmacologie, forensische wetenschappen, cosmetiek, biowetenschappen en nanotechnologie. Dit is bevorderd door recente ontwikkelingen in enerzijds vastestoflasers en anderzijds fotodetectoren met hoge resolutie, die gebaseerd zijn op CCD's (charge-coupled devices). Het onderzoek dat in dit proefschrift wordt beschreven heeft tot doel om bij te dragen tot een verdere verspreiding van deze technologie door de ontwikkeling mogelijk te maken van goedkope en draagbare Ramanspectrometers die geïntegreerd kunnen worden op een "chip". In het bijzonder is dit project gericht op de vervaardiging van geïntegreerde optische componenten die kunnen worden toegepast voor de detectie van Ramansignalen van biologische objecten zoals de menselijke huid en tanden.

Zulke spectrometersystemen zijn opgebouwd uit optische componenten om een exciterend lasersignaal aan het te onderzoeken specimen toe te voeren en het gegenereerde Ramansignaal op te vangen, een filter om ongewenste golflengten uit het lasersignaal te verwijderen, een filter om de lasergolflengte in het opgevangen signaal te onderdrukken, een golflengtesplitser -de eigenlijke Ramanspectrometeren, in sommige gevallen, polarisatiesplitsers. De benodigde lichtbron en fotodetectoren zijn in het bestek van dit proefschrift niet in detail bestudeerd.

In het onderzoek is gekozen voor siliciumoxynitride als materiaal voor de golfgeleidende laag in de geïntegreerde optische golfgeleiders waaruit de componenten worden opgebouwd. Dit materiaal is zeer geschikt voor toepassing in Ramanspectroscopie door zijn zeer lage optische verliezen over een breed golflengtespectrum en de grote ontwerpvrijheid die voortkomt uit de mogelijkheid om de brekingsindex via fabricageparameters nauwkeurig in te stellen binnen brede grenzen.

Een van de belangrijkste uitdagingen voor een geïntegreerde optische aanpak is het opvangen van zoveel mogelijk fotonen van het zeer zwakke Ramansignaal. Daartoe is bestudeerd in hoeverre kanaalgolfgeleiders de gebruikelijke glasvezelsondes kunnen vervangen. De geïntegreerde sondes blijken talrijke voordelen te hebben, in het bijzonder met betrekking tot de signaalopbrengst in vergelijking zowel met multimodale glasvezels met grote kerndiameter als met monomodale en multimodale glasvezels met kleine kerndiameter.

Eveneens is onderzocht of een enkele kanaalgolfgeleider wel de meest efficiënte integreerbare signaalcollector is. Hierbij is een nieuwe -inmiddels gepatenteerdegeïntegreerde optische component ontwikkeld die het mogelijk maakt om het 
excitatielicht te focusseren in een klein gebiedje op of vlak onder het oppervlak van het specimen en tegelijk het Ramansignaal op te vangen uit datzelfde gebiedje door middel van een confocale opstelling. Het collectierendement van deze component is een orde groter dan dat van een enkele kanaalgolfgeleider.

Ramanspectrometers zijn ontworpen en gerealiseerd voor de eerder genoemde toepassingen: detectie van water en de natuurlijke bevochtigingsfactor (natural moisturizing factor) in de buitenste laag van de huid -het stratum corneum- en voor de detectie van tandcariës in een vroeg stadium. De spectrometer is gebaseerd op een zogenaamde arrayed waveguide grating (AWG). Daarbij is een nieuwe vormgeving ontwikkeld waarin alle individuele golfgeleidende kanalen waaruit de AWG is opgebouwd identieke bochten maken. Dit maakt het mogelijk om systematische fasefouten te elimineren die in conventionele ontwerpen veroorzaakt worden door de verschillende bochten. De werking van deze spectrometer, de laser filters en de polarisatiesplitsers is gesimuleerd en door metingen gekarakteriseerd.

Tenslotte wordt experimenteel aangetoond dat de AWGs succesvol kunnen worden toegepast om gepolariseerde Ramanspectra te meten van menselijke tanden waarop plaatselijk beginnende cariës aanwezig was. De voor deze specifieke toepassing ontworpen AWG spectrometer heeft een golflengteresolutie van $0.2 \mathrm{~nm}$ en een vrij spectraal bereik van $22 \mathrm{~nm}$. De hiermee gemeten Ramanspectra bleken zeer goed overeen te komen met meetresultaten die verkregen zijn met klassieke laboratoriumspectrometers. De gemeten depolarisatieverhoudingen maken het mogelijk een duidelijk onderscheid te maken tussen gezond en carieus tandmateriaal, met dezelfde nauwkeurigheid als met (veel grotere) conventionele spectrometers. 


\section{Acknowledgements}

I would like to express my gratitude to all the people that accompanied me throughout the four years of my $\mathrm{PhD}$. These are the people that helped me overcome the difficulties encountered along the way and guided me towards the important achievements of this work.

In primis I wish to thank my promoters, Alfred Driessen and Markus Pollnau and assistant promoter René M. de Ridder. Thanks to Alfred, for giving me the opportunity to participate to this amazing project, for trusting in my capabilities right from the start and for his valuable guidance and support. I also thank Alfred for influencing me with his great enthusiasm and entrepreneurship which finally led towards the realization of a patent. An unimaginable achievement for me as a first year PhD student. In the same way I am extremely grateful to Markus, for guiding me throughout my $\mathrm{PhD}$ with insightful discussions and wise suggestions, for patiently revising my manuscripts, for being encouraging and always available to discuss new ideas and research directions. I equally wish to thank René, in particular for giving me continuous support and motivation, while at the same time being a strong critic and reviewer of my work. All this helped me understand numerous aspects of the way of doing research.

I would also like to acknowledge the rest of my graduation committee members: Prof. Peter Andersen from the Technical University of Denmark, Prof. Ton van Leeuwen from the University of Amsterdam, Prof. Vinod Subramaniam and Dr. Herman Offerhaus from the University of Twente for their valuable input and effort spent reviewing this thesis.

In addition I would like to thank my co-workers in the Photonic Integration Technology (PIT) Subgroup.

I am very thankful to Kerstin Wörhoff for her advice on modeling the optical properties of the $\mathrm{SiON}$ and $\mathrm{SiO}_{2}$ layers, and for depositing the layers. In addition I thank Kerstin for involving me in different projects such as the epiXnet project and the collaboration with the Center for Quantum Photonics of the University of Bristol. There I had the opportunity to interact with leading researchers such as Albert Peruzzo and Mark Thompson working at the cutting edge of science. I thank her for trusting in my capabilities and offering me a position as a researcher in the OCT project after the end of my PhD contract.

I wish to thank Fei Sun for supervising me in the first period of my $\mathrm{PhD}$, and for fabricating the devices together with Gabriel Sengo, to which I am very grateful for the explanations on the technical details of the device fabrication process, and for performing most of the cleanroom work. I wish to thank Imran Akca for the fruitful 
discussions on AWG design and characterization, and for being so kind in sending always beautiful presents to my daughter Asia.

I thank Anton Hollink, Meindert Dijkstra and Henk van Wolferen for their help in optimizing my measurement setups, and for supporting me through their vast technical knowledge. I am especially grateful to Anton, for his kindness, for his words of encouragement and support even during the most difficult moments of my $\mathrm{PhD}$, and I would like to let him know that I am close to him in this difficult moment of his life.

Thanks to Fehmi Civitci for his significant contributions in my first experiments and for the most interesting and inspiring discussions. To Chaitanya Dongre, for his strong friendship, for all the late night discussions on politics and on Italian movies especially those of Carlo Verdone. To So Van Pham, for the interesting discussions on cantilevers, Bragg gratings, and on benefits of meditation.

I also thank Alvin Baclig, Lin-P'ing Choo-Smith, Peter Caspers and Gerwin Puppels for their essential contributions to the Raman experiments. From the Raman pen project I also thank Marcel Hoekman for his valuable help with simulations and loss measurements and for once smoking with me on the metallic fire-escape balconies that surround the Hogekamp building (very dangerous). Thanks to Dmytry Podolsky, for his great positivity and encouraging words. In a particular way I thank Gerwin Puppels for being an excellent project leader always able to find the correct balance between the requirements of the industry and the needs of researchers.

I wish to thank all the other members of the Integrated Optical MicroSystems Group since they all helped me and inspired my research: Thanks to Hugo Hoekstra, Manfred Hammer, Sonia Garcia Blanco, Feridun Ay and Abu (Shanmugam Aravazhi), for giving me excellent advice and for their motivating attitude, to Dimitri Geskus for helping me out in many situations and for making every day seem bright and shiny. No, not by polishing, but through his amazing personality! To Laura Agazzi, for the Italian cakes, and the occasional Italian discussions that helped us recall technical terminology that otherwise we were starting to forget. To Edward Bernhardi, for introducing me to the fascinating world of foreign exchange where for a moment I thought I could easily become a millionaire, and for many inspiring discussions. To Marko van Dalfsen, for his kind help in many occasions where the Dutch language was a must and for the few but interesting discussions we had on the the middle east conflicts, to Lasse Kauppinen, for inspiring me to use a powerful open-source FDTD simulation program MEEP, that I'm still trying to master. To Jing Yang, for introducing the Ti:Sapphire setup to me for the first time, and to Murali Balakrishnan for first introducing to me the PM500 setup. Big thanks to the secretaries Rita ter Weele, Annitta David and Brigit Binkhorst for making everything work perfectly at all times, and for organizing fantastic group outings. 
I also wish to express my gratitude to Sesilia Kriswandhi, Remco Stoffer and Arjen Bakker for their help with the phoenix software which has been of vital importance for my simulations and mask designs.

Last but not least, I wish to dedicate this thesis to my family: to my parents Caterina and Hasan Ismail, and to my sisters Dunia and Hamdah as well as to my parents in law Marina and Giancarlo Marchetti. Thank you for always supporting me and encouraging me. In particular I dedicate this thesis to my wife Angela, that stood next to me in these four years making many renounces and sacrifices to enable me to complete my research in the smoothest way possible. Finally, I dedicate my thesis to my wonderful daughter Asia, that has been my muse in the past two years and that will continue to inspire me always.

Thank you All, Nur Ismail 


\section{List of publications}

\section{Peer reviewed international journals}

- Ismail, N., B. I. Akca, F. Sun, K. Wörhoff, R. M. de Ridder, M. Pollnau \& A. Driessen (2010) An integrated approach to laser delivery and confocal signal detection. Optics Letters, 35(16), 2741-2743.

- $\quad$ Nguyen, V. D., N. Ismail, F. Sun, K. Wörhoff, T. G. v. Leeuwen \& J. Kalkman (2010) SiON Integrated Optics Elliptic Couplers for Fizeau-Based Optical Coherence Tomography. Journal of Lightwave Technology, 28(19), 2836-2842.

- $\quad$ Peruzzo, A., M. Lobino, J. C. F. Matthews, N. Matsuda, A. Politi, K. Poulios, X.-Q. Zhou, Y. Lahini, N. Ismail, K. Wörhoff, Y. Bromberg, Y. Silberberg, M. G. Thompson \& J. L. O'Brien (2010) Quantum walks of correlated photons. Science, 329(5998), 1500-1503.

- Ismail, N., F. Civitci, K. Wörhoff, R. M. de Ridder, M. Pollnau \& A. Driessen (2011) Efficiency of integrated waveguide probes for the detection of light backscattered from weakly scattering media. Applied Optics, 50(6), 935-942.

- Ismail, N., F. Sun, K. Wörhoff, A. Driessen, R. M. de Ridder \& M. Pollnau (2011) Excitation and light collection from highly scattering media with integrated waveguides. Photonics Technology Letters, 23(5), 278-280.

- Ismail, N., F. Sun, G. Sengo, K. Wörhoff, A. Driessen, R. M. de Ridder \& M. Pollnau (2011) Improved arrayed-waveguide-grating layout avoiding systematic phase errors. Optics. Express, 19(9), 8781-8794.

- Akca, Akca, B. I., V. D. Nguyen, J. Kalkman, N. Ismail, G. Sengo, F. Sun, A. Driessen, T. G. v. Leeuwen, M. Pollnau, K. Wörhoff \& R. M. de Ridder (2011) Toward spectraldomain optical coherence tomography on a chip. Journal of Selected Topics in Quantum Electronics, PP(99), 1-11.

- Ismail, N., L.-P. Choo-Smith, K. Wörhoff, A. Driessen, A. C. Baclig, P. J. Caspers, G. J. Puppels, R. M. de Ridder \& M. Pollnau (2011) Raman spectroscopy with an integrated arrayed-waveguide grating. Optics Letters, 36(23), 4629-4631.

\section{Patent}

- Ismail, N., A. Driessen \& M. Pollnau (2009) Scattered light collector, light focusing apparatus, method for controlling the focus of a focused light source and method for measuring the light scattered or originating from a point of an object. WO/2010/126368. The Netherlands.

\section{Conference contributions}

- Ismail, N., E. J. Klein, G. Sengo \& A. Driessen (2008) Multi-waveguide based collector for the detection of backscattered light from highly scattering media. In X. J. M. Leijtens (ed.), 14th European Conference on Integrated Optics, pp. 119-122. Eindhoven University of Technology, Eindhoven, The Netherlands (Oral presentation). 
- Ismail, N., F. Sun, K. Wörhoff, M. Pollnau \& A. Driessen (2008) Detection of light backscattered from liquids by means of integrated optical waveguides. K. Wörhoff, L. Agazzi, N. Ismail \& X. Leijtens (eds.), 13th Annual Symposium of the IEEE/LEOS Benelux Chapter, pp. 27-29. University of Twente, Enschede, The netherlands (Oral presentation).

- Ismail, N., F. Sun, F. Civitci, K. Wörhoff, R. M. de Ridder, M. Pollnau \& A. Driessen (2009) Efficiency comparison between integrated optical waveguide probes and conventional fiber probes in the detection of backscattered light. 14th Annual Symposium of the IEEE Photonics Benelux Chapter, pp. 105-108. ASP-VUB Press, Brussels, Belgium (Poster).

- Ismail, N., F. Sun, F. Civitci, K. Wörhoff, R. M. de Ridder, M. Pollnau \& A. Driessen (2009) Use of integrated optical waveguide probes as an alternative to fiber probes for sensing of light backscattered from small volumes. 3rd EOS Topical Meeting on Optical Microsystems, Paper 2158. European Optical Society, Capri, Italy (Oral presentation).

- $\quad$ Nguyen, V. D., J. Kalkman, N. Ismail, F. Sun, K. Wörhoff, A. Driessen, M. Pollnau \& T. G. V. Leeuwen (2009) Design and characterization of SiON integrated optics components for optical coherence tomography. CLEO/Europe and EQEC 2009 Conference Digest, Paper CL.P.14 THU. Munich, Germany (Poster).

- $\quad$ Akca, B. I., N. Ismail, F. Sun, A. Driessen, K. Wörhoff, M. Pollnau, R. M. de Ridder, V. D. Nguyen, J. Kalkman \& T. G. v. Leeuwen (2010) Integrated AWG spectrometer for onchip optical coherence tomography and Raman spectroscopy. 15th European Conference on Integrated Optics, Paper WeB1. IEEE Photonics Society, Cambridge, UK (Oral presentation).

- $\quad$ Akca, B. I., N. Ismail, F. Sun, V. D. Nguyen, J. Kalkman, T. G. v. Leeuwen, A. Driessen, K. Wörhoff, M. Pollnau \& R. M. de Ridder (2010) Integrated arrayed waveguide grating spectrometer for on-chip optical coherence tomography. Conference on Lasers and Electro-Optics, Paper JWA66. San José, California. Technical Digest (Optical Society of America, Washington, DC 2010) (Poster).

- Ismail, N., B. I. Akca, F. Sun, K. Wörhoff, R. M. de Ridder, M. Pollnau \& A. Driessen (2010) Arrayed-waveguide-grating light collector for on-chip spectroscopy. 15th European Conference on Integrated Optics, Paper WeB5. IEEE Photonics Society, Cambridge, UK (Oral presentation).

- $\quad$ Ismail, N., A. C. Baclig, P. J. Caspers, F. Sun, K. Wörhoff, R. M. de Ridder, M. Pollnau \& A. Driessen (2010) Design of low-loss arrayed waveguide gratings for applications in integrated Raman spectroscopy. Conference on Lasers and Electro-Optics, Paper CFA7. San José, California, USA. Technical Digest (Optical Society of America, Washington, DC 2010) (Oral presentation).

- Ismail, N., F. Sun, F. Civitci, K. Wörhoff, R. M. de Ridder, M. Pollnau \& A. Driessen (2010) Efficiency of integrated waveguide probes in the detection of fluorescence and backscattered light. Optical Fibers and Sensors for Medical Diagnostics and Treatment Applications X, Paper 755903. SPIE, San Francisco, California, USA (Oral presentation).

- $\quad$ Nguyen, V. D., T. G. v. Leeuwen, J. Kalkman, B. I. Akca, N. Ismail, F. Sun, A. Driessen, K. Wörhoff, M. Pollnau \& R. M. de Ridder (2010) SiON integrated optics elliptic couplers for Fizeau-based optical coherence tomography. 15th European Conference on 
Integrated Optics, Paper ThP23. Cambridge, UK (Poster).

- $\quad$ Akca, B.I., N. Ismail, F. Sun, A. Driessen, K. Wörhoff, M. Pollnau, R.M. de Ridder, V.D. Nguyen, J. Kalkman \& T.G. van Leeuwen (2010) Integrated AWG spectrometer for onchip optical coherence tomography and Raman spectroscopy, 15th European Conference on Integrated Optics, Paper WeB1. Cambridge, UK (Poster).

- $\quad$ Peruzzo, A., J. Matthews, A. Politi, M. Lobino, X.-Q. Zhou, M. Thompson, J. O'Brien, N. Matsuda, N. Ismail, K. Worhoff, Y. Bromberg, Y. Lahini \& Y. Silberberg (2010) Quantum random walks circuits with photonic waveguides. 15th European Conference on Integrated Optics, Paper FrJB1. IEEE Photonics Society, Cambridge, UK (Oral presentation).

- Ismail, N., F. Sun, G. Sengo, K. Wörhoff, A. Driessen, R. M. de Ridder \& M. Pollnau (2011) Broadband polarization-insensitive arrayed waveguide gratings for Raman spectroscopy. Conference on Lasers and Electro-Optics. Baltimore, MA, USA. Technical Digest (Optical Society of America, Washington, DC 2011). (Oral presentation).

- Akca, B.I., N. Ismail, F. Sun, A. Driessen, K. Wörhoff, M. Pollnau \& R.M. de Ridder (2011) High-resolution integrated spectrometers in silicon-oxynitride. Conference on Lasers and Electro-Optics, Paper JWA65. Baltimore, MA, USA, Technical Digest (Optical Society of America, Washington, DC 2011). (Oral presentation).

- Ismail, N., F. Sun, K. Wörhoff, A. Driessen, R. M. de Ridder \& M. Pollnau (2011) Integrated Arrayed Waveguide Grating Spectrometer for Measuring Raman Spectra. Conference on Lasers and Electro-Optics Europe. Munich, Germany (Oral presentation).

- Ismail, N., K. Wörhoff, L.-P. Choo-Smith, A. C. Baclig, P. J. Caspers, G. J. Puppels, A. Driessen, R. M. de Ridder \& M. Pollnau (2011) On-chip Raman spectrometer for the detection of early dental caries. International Quantum Electronics Conference and Conference on Lasers and Electro-Optics Pacific Rim. Sydney, Australia. Conference Handbook, p. 76, Paper 2700-PO-64. (Poster).

- Wörhoff, K., N. Ismail, B. I. Akça, M. Pollnau \& R. M. d. Ridder (2011) Silicon oxynitride technology for integrated optical solutions in biomedical applications. 13th International Conference on Transparent Optical Networks, 7th workshop on Photonic Integrated Components \& Applications. Session Th.A4, Paper Th.A4.6, p. 24. Stockholm, Sweden. (Oral presentation)

- Pollnau, M., B. I. Akca, N. Ismail, C. Dongre, S. V. Pham, K. Wörhoff, R. M. d. Ridder \& H. J. W. M. Hoekstra (2011) Biophotonic sensors on a microchip for trace-gas detection, DNA and enzyme analysis, Raman spectroscopy, and optical coherence tomography. Joint Annual Meeting of the Swiss Physical Society and Austrian Physical Society. Ecole Polytechnique Federale de Lausanne. Bulletin SPG/SSP, Vol. 28, Paper 213. Lausanne, Switzerland. (Oral presentation).

- Ismail, N., L.-P. Choo-Smith, K. Wörhoff, A. Driessen, A. C. Baclig, P. J. Caspers, G. J. Puppels, R. M. de Ridder \& M. Pollnau (2011) Raman spectroscopy of human teeth using integrated optical spectrometers. P. Bienstman, G. Morthier, G. Roelkens \& Marie Verbist (eds.), 16th Annual Symposium of the IEEE Photonics Benelux Chapter, pp. 6568. Gent University, Gent, Belgium (Oral presentation). 